The Astrophysical Journal Supplement Series, 68:91-127, 1988 October

(C) 1988. The American Astronomical Society. All rights reserved. Printed in U.S.A.

\title{
A CATALOG OF IRAS OBSERVATIONS OF LARGE OPTICAL GALAXIES
}

\author{
W. Rice, ${ }^{1}$ Carol J. Lonsdale, ${ }^{1}$ B. T. Solfer,,${ }^{1,2}$ G. Neugebauer,${ }^{2}$ E. L. Kopan, ${ }^{1}$ \\ LaWrence A. Lloyd, ${ }^{1}$ T. DE JONG ${ }^{3}$ and H. J. Habing ${ }^{4}$ \\ Received 1987 June 22; accepted 1988 January 11
}

\begin{abstract}
A catalog is presented of the IRAS observations of 85 galaxies listed in the Second Reference Catalogue of Bright Galaxies with blue-light isophotal diameters greater than 8 '. "Total" flux densities at 12, 25, 60, and 100 $\mu \mathrm{m}$, obtained from spatial maps constructed from co-added IRAS detector data, are reported.

Infrared brightness profiles of the detected galaxies and infrared surface brightness contour maps of the galaxies for which structural features were resolved are displayed in an atlas. A far-infrared classification scheme based on the degree of central concentration and spatial structure of the $60 \mu \mathrm{m}$ emission of the best-resolved galaxies is proposed. The $60 \mu \mathrm{m}$ and blue-light isophotal diameters of the largest galaxies are compared.
\end{abstract}

Subject headings: galaxies: photometry - galaxies: structure - infrared: sources

\section{INTRODUCTION}

Of the approximately 22,000 galaxies detected in the Infrared Astronomical Satellite (IRAS) all-sky survey (Chester 1985), about 100 of the nearest galaxies have large enough apparent optical extents to show spatial structure at the arcminute angular resolution of the IRAS beam. The IRAS observations of these optically large galaxies present unique data for comparing the spatial distribution of the mid- and far-infrared emission with the distributions of the stars and the gas within a variety of galactic disks. Such studies are important for determining the contribution of the various possible infrared emitting sources to the global infrared emission of external galaxies and for determining how the global infrared emission relates to stellar processes on a galactic scale. Clearly, these results are vital for interpreting the thousands of unresolved IRAS galaxy detections.

This catalog presents the IRAS observations of the optically large galaxies likely to show structural features at the IRAS resolution. The catalog sample consists of all galaxies observed by IRAS, which are listed in the Second Reference Catalogue of Bright Galaxies (de Vaucouleurs, de Vaucouleurs, and Corwin 1976, hereafter RC2) with apparent blue-light isophotal diameters greater than 8'. "Total" flux densities at $12,25,60$, and $100 \mu \mathrm{m}$ are reported, and an atlas of infrared brightness profile plots of the detected galaxies and infrared surface brightness contour maps of those galaxies for which structure was observed is presented. This catalog complements the existing IRAS catalogs, providing global flux densities, obtained from spatial maps constructed from co-added $I R A S$ detector data, of the large galaxies for which the entries in the IRAS Point Source Catalog (1985, hereafter PSC) and

\footnotetext{
${ }^{1}$ Infrared Processing and Analysis Center, California Institute of Technology.

${ }^{2}$ Division of Physics, Mathematics, and Astronomy, California Institute of Technology.

${ }^{3}$ Universiteit van Amsterdam.

${ }^{4}$ Sterrewacht, Leiden.
}

the IRAS Small Scale Structures Catalog (1986, hereafter SSSC) underestimate the total flux density. [The PSC flux densities are total emission measurements for sources smaller than $\sim 2^{\prime}$ (FWHM), while the SSSC flux densities represent the total emission for sources less than $\sim 8^{\prime}$ (FWHM).]

The selection of the "large optical galaxy" sample is described in $\S$ II. In $\S$ III we describe the IRAS observations, the data processing, and data measurement techniques. Section IV reports the total IRAS flux densities and integrated infrared emission properties of the sample. The atlas, including maps of the best-resolved galaxies, is presented in $\S \mathrm{V}$. A brief preliminary analysis of the catalog is given in $\S \mathrm{VI}$. A detailed study of the spatial distribution of the infrared emission and colors of the large galaxies will be presented elsewhere (Rice 1988, hereafter Paper II). Additional galaxies with blue-light isophotal diameters greater than $8^{\prime}$ as listed in other catalogs are discussed in the Appendix.

\section{THE LARGE OPTICAL GALAXY SAMPLE}

Eighty-six galaxies in RC2 are listed with blue-light isophotal major diameters $\left(D_{25}\right)$ greater than $8^{\prime}$. All but one of these galaxies, the dE5 pec galaxy A1110+22 (= Leo B), was observed by $I R A S$. The 85 observed galaxies are presented in Table 1. Column (1) is the galaxy RC2 identification-the NGC (N), IC (I), or anonymous coordinate (A) designation. An alternate designation - the Messier (M) catalog number, the traditional name, or the David Dunlap Observatory (D) designation (van den Bergh 1959, 1966) of unnamed dwarf systems - is given in column (2). Columns (3) and (4) are the optically determined equatorial coordinates of the galaxy (equinox 1950) as listed in RC2. The Galactic coordinates of the galaxy are given in columns (5) and (6). Column (7) gives the galaxy revised Hubble morphological type as listed in the catalog of Sandage and Tammann (1981, hereafter RSA) or, for galaxies not contained in RSA, the morphological type, in brackets, as reported in RC2. The morphological classification as coded in RC2 as a $T$ index and bar parameter letter is listed in column (8). The RC2 apparent blue light major $\left(D_{25}\right)$ 
and minor $\left(d_{25}\right)$ isophotal diameters in arcminutes are given in columns (9) and (10). Column (11) is the distance in megaparsecs based on RSA velocities and distance moduli (RSA, Table 2), or velocities listed in the sources cited and corrected, if necessary, using the precepts in RSA, and using a Hubble constant of $H_{0}=50 \mathrm{~km} \mathrm{~s}^{-1} \mathrm{Mpc}^{-1}$.

\section{IRAS OBSERVATIONS AND DATA REDUCTION}

The IRAS data used to construct spatial maps of the sample galaxies have been taken from the high-sensitivity pointed observations (Neugebauer et al. 1984) whenever possible. The pointed observations were multi-leg raster pattern

TABLE 1

LaRge Optical Galaxies ObSERved ${ }^{\mathrm{a}}$ IN THE IRAS MisSiON

\begin{tabular}{|c|c|c|c|c|c|c|c|c|c|c|c|c|}
\hline $\begin{array}{c}\mathrm{RC} 2 \\
\text { Name } \\
(1)\end{array}$ & $\begin{array}{l}\text { Other } \\
\text { Name } \\
(2)\end{array}$ & & $\begin{array}{r}\text { R.A } \\
(3)\end{array}$ & & $\begin{array}{l}\text { 50) Dec. } \\
\text { (4) }\end{array}$ & $\begin{array}{c}l \\
(5)\end{array}$ & $\begin{array}{c}b \\
(6)\end{array}$ & $\begin{array}{c}\text { Type } \\
(7)\end{array}$ & $\begin{array}{c}T \\
(8)\end{array}$ & $\begin{array}{l}D_{25} \\
(9)\end{array}$ & $\begin{array}{c}d_{25} \\
(10)\end{array}$ & $\begin{array}{c}\text { Distance }^{\mathrm{b}} \\
(\mathrm{Mpc}) \\
(11)\end{array}$ \\
\hline N45 & D223 & $0^{\mathrm{h}}$ & ${ }^{\mathrm{h}} 11^{\mathrm{m}}$ & ${ }^{n} 31^{8} .8$ & $-23^{\circ} 27^{\prime} 36^{\prime \prime}$ & $55^{\circ} .9$ & $-80^{\circ} .7$ & $\operatorname{Scd}(s)$ & $8 \mathrm{~A}$ & $8: 1$ & $5: 7$ & 10.66 \\
\hline N55 & & 0 & 12 & 24.0 & -392800 & 332.9 & -75.7 & $\mathrm{Sc}$ & $9 \mathrm{~B}$ & 32.3 & 6.4 & 3.16 \\
\hline N134 & & 0 & 27 & 54.0 & $-33 \quad 3200$ & 338.2 & -82.4 & $\mathrm{Sbc}(\mathrm{s})$ & $4 X$ & 8.1 & 2.6 & 31.88 \\
\hline N147 & D3 & 0 & 30 & 27.6 & $48 \quad 13 \quad 48$ & 119.8 & -14.3 & $\mathrm{dE5}$ & -5 & 12.9 & 8.1 & 0.73 \\
\hline N185 & & 0 & 36 & 11.4 & $\begin{array}{lll}48 & 03 & 42\end{array}$ & 120.8 & -14.5 & dE3 pec & -5 & 11.5 & 9.8 & 0.73 \\
\hline N205 & & 0 & 37 & 38.4 & 412454 & 120.7 & -21.1 & S0/E5 pec & -5 & 17.4 & 9.8 & 0.73 \\
\hline N224 & M31 & 0 & 40 & 00.0 & $40 \quad 5942$ & 121.2 & -21.6 & $\mathrm{Sb}$ & $3 \mathrm{~A}$ & 177.8 & 63.1 & 0.73 \\
\hline N247 & & 0 & 44 & 39.6 & -210200 & 113.9 & -83.6 & $\mathrm{Sc}(\mathrm{s})$ & $7 \mathrm{X}$ & 19.9 & 7.4 & 3.16 \\
\hline N253 & & 0 & 45 & 07.8 & $-25 \quad 3342$ & 97.6 & -88.0 & $\mathrm{Sc}(\mathrm{s})$ & $5 X$ & 25.1 & 7.4 & 4.17 \\
\hline A $0051-73$ & SMC & 0 & 51 & 00.0 & -730600 & 302.8 & -44.3 & $\mathrm{Im}$ & $9 \mathrm{~B}$ & 281.8 & 158.5 & 0.078 \\
\hline N300 & & 0 & 52 & 31.2 & -375724 & 299.2 & -79.4 & $\mathrm{Sc}$ & $7 \mathrm{~A}$ & 19.9 & 14.8 & 2.40 \\
\hline I1613 & D8 & 1 & 02 & 13.2 & 15100 & 129.7 & -60.6 & {$[\operatorname{Im}(\mathrm{s})]$} & $10 \mathrm{X}$ & 12.0 & 11.2 & $0.85^{(1)}$ \\
\hline N598 & M33 & 1 & 31 & 03.0 & $30 \quad 2354$ & 133.6 & -31.3 & $\mathrm{Sc}(\mathrm{s})$ & $5 \mathrm{~A}$ & 61.6 & 38.9 & 0.90 \\
\hline N628 & M74 & 1 & 34 & 00.6 & 153136 & 138.6 & -45.7 & $\mathrm{Sc}(\mathrm{s})$ & $5 \mathrm{~A}$ & 10.2 & 9.5 & 17.22 \\
\hline N660 & & 1 & 40 & 21.0 & $13 \quad 2318$ & 141.6 & -47.4 & {$[\mathrm{Sa}]$} & $1 \mathrm{~B}$ & 9.1 & 4.1 & $4^{(2)}$ \\
\hline N891 & & 2 & 19 & 24.6 & $42 \quad 07 \quad 12$ & 140.4 & -17.4 & $\mathrm{Sb}$ & $5 \mathrm{~A}$ & 13.5 & 2.8 & 15.58 \\
\hline N925 & & 2 & 24 & 16.8 & $33 \quad 2124$ & 144.9 & -25.2 & $\mathrm{SBc}(\mathrm{s})$ & $7 \mathrm{X}$ & 9.8 & 6.0 & 15.84 \\
\hline N1023 & & 2 & 37 & 16.2 & 385054 & 145.0 & -19.1 & $\mathrm{SBO}_{1}(5)$ & $-2 \mathrm{~B}$ & 8.7 & 3.3 & 17.90 \\
\hline A $0237-34$ & Fornax Sys. & 2 & 37 & 50.4 & -344424 & 237.3 & -65.7 & {$[\mathrm{EOp}]$} & -5 & 19.9 & 13.8 & $0.16^{(1)}$ \\
\hline N 1097 & & 2 & 44 & 11.4 & -302906 & 226.9 & -64.7 & $\mathrm{RSBbc}(\mathrm{rs})$ & $3 \mathrm{~B}$ & 9.3 & 6.6 & 25.68 \\
\hline $\mathrm{N} 1291$ & & 3 & 15 & 28.8 & $-41 \quad 1830$ & 247.6 & -57.0 & $\mathrm{SBa}$ & $\mathrm{OB}$ & 10.5 & 9.1 & 14.76 \\
\hline $\mathrm{N} 1313$ & & 3 & 17 & 38.9 & -664042 & 283.4 & -44.6 & $\mathrm{SBc}(\mathrm{s})$ & $7 \mathrm{~B}$ & 8.5 & 6.6 & 5.22 \\
\hline N1365 & & 3 & 31 & 42.0 & $\begin{array}{lll}-36 & 18 & 18\end{array}$ & 238.0 & -54.6 & $\mathrm{SBb}(\mathrm{s})$ & $3 B$ & 9.8 & 5.5 & 31.24 \\
\hline I 342 & & 3 & 41 & 57.6 & 675624 & 138.2 & 10.6 & {$[\mathrm{~S}(\mathrm{rs}) \mathrm{cd}]$} & $6 \mathrm{X}$ & 17.8 & 17.4 & $4.68^{(1)}$ \\
\hline N1448 & & 3 & 42 & 52.8 & $-44 \quad 4800$ & 251.5 & -51.4 & Sc: & $6 \mathrm{~A}$ & 8.1 & 1.8 & 20.76 \\
\hline N1560 & & 4 & 27 & 03.6 & $\begin{array}{lll}71 & 46 & 12\end{array}$ & 138.4 & 16.0 & {$[\mathrm{Sd}(\mathrm{s})]$} & $7 \mathrm{~A}$ & 9.8 & 2.0 & $4.68^{(1)}$ \\
\hline A0524-69 & LMC & 5 & 24 & 00.0 & -694800 & 280.5 & -32.9 & $\mathrm{SBm}$ & $9 \mathrm{~B}$ & 645.6 & 549.5 & 0.057 \\
\hline $\mathrm{N} 2403$ & & 7 & 32 & 03.0 & $65 \quad 4242$ & 150.6 & 29.2 & $\mathrm{Sc}(\mathrm{s})$ & $6 \mathrm{X}$ & 17.8 & 11.0 & 3.57 \\
\hline N2683 & & 8 & 49 & 34.8 & 333630 & 190.5 & 38.8 & $\mathrm{Sb}$ & $3 \mathrm{~A}$ & 9.3 & 2.5 & 7.98 \\
\hline N2841 & & 9 & 18 & 34.8 & $\begin{array}{lll}51 & 11 & 18\end{array}$ & 166.9 & 44.1 & $\mathrm{Sb}$ & $3 \mathrm{~A}$ & 8.1 & 3.8 & 14.28 \\
\hline N2903 & & 9 & 29 & 20.4 & $21 \quad 43 \quad 12$ & 208.7 & 44.5 & $\mathrm{Sc}(\mathrm{s})$ & $4 \mathrm{X}$ & 12.6 & 6.6 & 9.44 \\
\hline N2997 & & 9 & 43 & 27.6 & -305736 & 262.6 & 16.8 & $\mathrm{Sc}(\mathrm{s})$ & $5 \mathrm{X}$ & 8.1 & 6.4 & 15.98 \\
\hline N3031 & M81 & 9 & 51 & 30.0 & $\begin{array}{lll}69 & 18 & 18\end{array}$ & 142.1 & 40.9 & $\mathrm{Sb}(\mathrm{r})$ & $2 \mathrm{~A}$ & 25.7 & 14.1 & 3.57 \\
\hline N3034 & M82 & 9 & 51 & 40.8 & 695454 & 141.4 & 40.6 & Amorphous & $0:$ & 11.2 & 4.6 & 3.57 \\
\hline N3109 & D236 & 10 & 00 & 46.8 & $-25 \quad 5448$ & 262.1 & 23.1 & $\mathrm{Sm}$ & $9 \mathrm{~B}$ & 14.4 & 3.5 & 2.58 \\
\hline N3115 & & 10 & 02 & 44.4 & -072830 & 247.8 & 36.8 & $\mathrm{~S}_{1}(7)$ & -3 & 8.3 & 3.2 & 8.74 \\
\hline A $1005+12$ & Leo I & 10 & 05 & 46.2 & $12 \quad 3312$ & 226.0 & 49.1 & [E3] & -4 & 10.7 & 8.3 & $0.12^{(1)}$ \\
\hline N3198 & & 10 & 16 & 52.2 & 454800 & 171.2 & 54.8 & $\mathrm{Sc}(\mathrm{rs})$ & $5 \mathrm{~B}$ & 8.3 & 3.7 & 14.04 \\
\hline $\mathrm{I} 2574$ & D81 & 10 & 24 & 40.2 & 684006 & 140.2 & 43.6 & {$[\mathrm{Sm}(\mathrm{s})]$} & $9 \mathrm{X}$ & 12.3 & 5.9 & $3.50^{(1)}$ \\
\hline N3521 & & 11 & 03 & 15.6 & 01412 & 255.5 & 52.8 & $\mathrm{Sb}(\mathrm{s})$ & $4 \mathrm{X}$ & 9.5 & 5.0 & 12.54 \\
\hline N3556 & & 11 & 08 & 36.6 & $55 \quad 5642$ & 148.3 & 56.3 & $\mathrm{Sc}(\mathrm{s})$ & $6 \mathrm{~B}$ & 8.3 & 2.5 & 15.80 \\
\hline N3621 & & 11 & 15 & 50.4 & $-3232 \quad 24$ & 281.2 & 26.1 & $\mathrm{Sc}(\mathrm{s})$ & $7 \mathrm{~A}$ & 10.0 & 6.5 & 8.70 \\
\hline N3623 & M65 & 11 & 16 & 18.6 & 132154 & 241.3 & 64.2 & $\mathrm{Sa}(\mathrm{s})$ & $1 \mathrm{X}$ & 10.0 & 3.3 & 13.50 \\
\hline N3627 & M66 & 11 & 17 & 38.4 & $13 \quad 1548$ & 242.0 & 64.4 & $\mathrm{Sb}(\mathrm{s})$ & $3 \mathrm{X}$ & 8.7 & 4.4 & 11.86 \\
\hline N3628 & & 11 & 17 & 39.6 & 135206 & 240.8 & 64.8 & $\mathrm{Sbc}$ & 3 & 14.8 & 3.6 & 14.38 \\
\hline
\end{tabular}


TABLE $1-$ Continued

\begin{tabular}{|c|c|c|c|c|c|c|c|c|c|c|c|}
\hline $\begin{array}{c}\text { RC2 } \\
\text { Name } \\
(1) \\
\end{array}$ & $\begin{array}{c}\text { Other } \\
\text { Name } \\
(2)\end{array}$ & $\begin{array}{r}\text { R.A } \\
(3) \\
\end{array}$ & A. $(195$ & $\begin{array}{c}\text { 50) Dec. } \\
(4)\end{array}$ & $\begin{array}{c}l \\
(5)\end{array}$ & $\begin{array}{c}b \\
(6)\end{array}$ & $\begin{array}{c}\text { Type } \\
(7)\end{array}$ & $\begin{array}{c}T \\
(8) \\
\end{array}$ & $\begin{array}{l}D_{25} \\
(9)\end{array}$ & $\begin{array}{c}d_{25} \\
(10)\end{array}$ & $\begin{array}{c}\text { Distance } \\
(\mathrm{Mpc}) \\
(11)\end{array}$ \\
\hline N3718 & & $11^{\mathrm{h}} 29^{\mathrm{m}}$ & ${ }^{n} 49^{8} .8$ & $53^{\circ} 20^{\prime} 42^{\prime \prime}$ & $147^{\circ} .0$ & $60^{\circ} .2$ & Sa pec? & 1 & 8.7 & 4.5 & 21.64 \\
\hline N4192 & M98 & 1211 & 15.6 & $15 \quad 1048$ & 265.4 & 75.0 & Sb: & $2 \mathrm{X}$ & 9.5 & 3.1 & 21.88 \\
\hline N4216 & & 1213 & 21.0 & 132524 & 270.5 & 73.7 & $\mathrm{Sb}(\mathrm{s})$ & $3 \mathrm{X}$ & 8.3 & 2.2 & 21.88 \\
\hline N4236 & & 1214 & 19.2 & 694500 & 127.4 & 47.4 & SBd & $8 B$ & 18.6 & 6.9 & 3.14 \\
\hline N4244 & & 1214 & 59.4 & $\begin{array}{lll}38 & 05 & 12\end{array}$ & 154.6 & 77.2 & Scd & $6 \mathrm{~A}$ & 16.2 & 2.5 & 4.98 \\
\hline N4258 & & 1216 & 29.4 & 473500 & 138.3 & 68.8 & $\mathrm{Sb}(\mathrm{s})$ & $4 X$ & 18.2 & 5.0 & 10.40 \\
\hline N4395 & & 1223 & 19.8 & 334930 & 162.1 & 81.5 & $\mathrm{Sd}$ & $9 \mathrm{~A}$ & 12.9 & 11.0 & 6.08 \\
\hline N4438 & & 1225 & 13.8 & 131706 & 280.3 & 74.8 & $\mathrm{Sb}$ & $0 A$ & 9.3 & 3.9 & 21.88 \\
\hline N4472 & M49 & 1227 & 14.4 & 81642 & 286.9 & 70.2 & $\mathrm{E} 1 / \mathrm{SO}_{1}(1)$ & -5 & 8.9 & 7.4 & 21.88 \\
\hline $\mathrm{N} 4517$ & & 1230 & 12.0 & 02318 & 292.7 & 62.6 & $\mathrm{Sc}$ & $6 \mathrm{~A}$ & 10.2 & 1.9 & 15.93 \\
\hline N4559 & & 1233 & 29.4 & $28 \quad 1406$ & 198.4 & 86.5 & $\mathrm{Sc}(\mathrm{s})$ & $6 \mathrm{X}$ & 10.5 & 4.9 & 15.42 \\
\hline N4565 & & 1233 & 51.6 & 261536 & 230.8 & 86.4 & $\mathrm{Sb}$ & $3 \mathrm{~A}$ & 16.2 & 2.8 & 23.42 \\
\hline N4569 & M90 & 1234 & 18.6 & $13 \quad 2624$ & 288.5 & 75.6 & $\operatorname{Sab}(\mathrm{s})$ & $2 X$ & 9.5 & 4.7 & 21.88 \\
\hline N4594 & M104 & 1237 & 22.8 & -112100 & 298.5 & 51.1 & $\mathrm{Sa}^{+} / \mathrm{Sb}^{-}$ & $1 \mathrm{~A}$ & 8.9 & 4.1 & 17.46 \\
\hline N4631 & & 1239 & 40.8 & 324848 & 142.8 & 84.2 & $\mathrm{Sc}$ & $7 \mathrm{~B}$ & 15.1 & 3.3 & 12.12 \\
\hline N4656 & & 1241 & 31.8 & 322630 & 140.3 & 84.7 & $\mathrm{Im}$ & $9 \mathrm{~B}$ & 13.8 & 3.3 & 12.48 \\
\hline N4725 & & 1248 & 00.0 & 254630 & 295.1 & 88.4 & $\mathrm{Sb} / \mathrm{SBb}(\mathrm{r})$ & $2 X$ & 10.9 & 7.9 & 23.34 \\
\hline N4736 & M94 & 1248 & 31.8 & 412336 & 123.4 & 76.0 & $\mathrm{RSab}(\mathrm{s})$ & $2 \mathrm{~A}$ & 10.9 & 9.1 & 6.90 \\
\hline N4762 & & 1250 & 25.2 & 113006 & 304.3 & 74.1 & $\mathrm{SO}_{1}(10)$ & $-2 \mathrm{~B}$ & 8.7 & 1.6 & 21.88 \\
\hline $\mathrm{N} 4826$ & M64 & 1254 & 16.8 & 215706 & 315.7 & 84.4 & $\mathrm{Sab}(\mathrm{s})$ & $2 \mathrm{~A}$ & 9.3 & 5.4 & 7.00 \\
\hline N4945 & & 1302 & 31.8 & $-49 \quad 1200$ & 305.3 & 13.3 & $\mathrm{Sc}$ & $6 \mathrm{~B}$ & 19.9 & 4.4 & 6.92 \\
\hline N5033 & & 1311 & 08.4 & $36 \quad 5148$ & 98.1 & 79.4 & $\operatorname{Sbc}(\mathrm{s})$ & $5 \mathrm{~A}$ & 10.5 & 5.6 & 17.94 \\
\hline N5055 & M63 & 1313 & 35.4 & $42 \quad 1748$ & 106.0 & 74.3 & $\operatorname{Sbc}(\mathrm{s})$ & $4 \mathrm{~A}$ & 12.3 & 7.6 & 11.00 \\
\hline N5102 & & 1319 & 07.2 & -362206 & 309.7 & 25.8 & $\mathrm{SO}_{1}(5)$ & $-3 \mathrm{~A}$ & 9.3 & 3.5 & 6.92 \\
\hline N5128 & Cen A & 1322 & 31.8 & -424530 & 309.5 & 19.4 & So+S pec & -2 & 18.2 & 14.5 & 6.92 \\
\hline N5170 & & 1327 & 07.2 & -174224 & 315.7 & 44.0 & $\mathrm{Sb}:$ & $5 \mathrm{~A}$ & 8.1 & 1.3 & 25.70 \\
\hline N5194 & M51 & 1327 & 45.6 & $\begin{array}{lll}47 & 27 & 18\end{array}$ & 104.9 & 68.6 & $\operatorname{Sbc}(\mathrm{s})$ & $4 \mathrm{~A}$ & 10.9 & 7.7 & 10.82 \\
\hline N5236 & M83 & 1334 & 10.2 & -293648 & 314.6 & 32.0 & $\mathrm{SBc}(\mathrm{s})$ & $5 \mathrm{X}$ & 11.2 & 10.2 & 9.92 \\
\hline N5457 & M101 & 1401 & 27.6 & 543536 & 102.0 & 59.8 & $\mathrm{Sc}(\mathrm{s})$ & $6 \mathrm{X}$ & 26.9 & 26.3 & 7.59 \\
\hline $\mathrm{A} 1508+67$ & $\mathrm{U} \mathrm{Mi}$ & 1508 & 12.0 & 672300 & 105.0 & 44.8 & [E4] & -5 & 32.3 & 20.9 & $0.090^{(1)}$ \\
\hline N5907 & & 1514 & 36.6 & 563024 & 91.6 & 51.1 & $\mathrm{Sc}$ & $5 \mathrm{~A}$ & 12.3 & 1.8 & 15.58 \\
\hline $\mathrm{A} 1719+57$ & Draco Sys. & 1719 & 15.2 & 575730 & 86.4 & 34.7 & {$[\mathrm{E} 0 \mathrm{p}]$} & -5 & 39.8 & 24.5 & $0.095^{(1)}$ \\
\hline N6744 & & 1905 & 01.7 & $\begin{array}{lll}-63 & 56 & 18\end{array}$ & 332.2 & -26.1 & $\operatorname{Sbc}(\mathrm{r})$ & $4 \mathrm{X}$ & 15.5 & 10.2 & 13.26 \\
\hline N6822 & D209 & .1942 & 07.1 & $-14 \quad 5542$ & 25.3 & -18.4 & $\operatorname{Im}$ & $10 \mathrm{~B}$ & 10.2 & 9.5 & 0.68 \\
\hline N6946 & & 2033 & 47.9 & 595900 & 95.7 & 11.7 & $\mathrm{Sc}(\mathrm{s})$ & $6 \mathrm{X}$ & 10.9 & 9.7 & 6.72 \\
\hline $\mathrm{I}^{2} 201^{\mathrm{c}}$ & & 2217 & 55.0 & $-46 \quad 1700$ & 350.2 & -54.9 & SBcd & $6 \mathrm{~B}$ & 8.5 & 4.3 & 14.56 \\
\hline N7331 & & 2234 & 47.2 & 340930 & 93.7 & -20.7 & $\mathrm{Sb}(\mathrm{rs})$ & $4 \mathrm{~A}$ & 10.7 & 4.0 & 22.28 \\
\hline N7640 & & 2319 & 43.1 & $40 \quad 3412$ & 105.2 & -18.9 & $\operatorname{Sbc}(\mathbf{s}):$ & $5 \mathrm{~B}$ & 10.7 & 2.5 & 13.38 \\
\hline N7793 & & 2355 & 15.5 & -325206 & 4.5 & -77.2 & $\operatorname{Sd}(\mathrm{s})$ & $8 \mathrm{~A}$ & 9.1 & 6.6 & 6.62 \\
\hline A2359-15 & WLM & 2359 & 23.9 & -154436 & 75.8 & -73.6 & {$[\operatorname{Im}(\mathbf{s})]$} & $10 \mathrm{~B}$ & 10.2 & 4.2 & $1.60^{(1)}$ \\
\hline
\end{tabular}

${ }^{\mathrm{a}} \mathrm{A} 1110+22$ ( = Leo B), a dE5 pec galaxy with an RC2 diameter of 14.4, was not observed in the IRAS mission.

${ }^{b}$ Distance sources: RSA; (1) Kraan-Korteweg and Tammann 1979; (2) Huchra et al. 1983.

'Optical position from RSA.

scans of the IRAS survey detector array covering small areas of the sky (typically $1.5 \times 0.5$ ) centered on the targeted objects.

For the 36 galaxies of the sample for which pointed observations were not performed, maps were constructed from co-added all-sky survey data. The coverage of these fields was a minimum of four survey scans (or 2 hours confirming scans). These data were processed in the same way as the pointed observation data.
In the following, the in-scan direction refers to the scanning direction of the IRAS telescope and the cross-scan direction refers to the direction orthogonal to the scanning direction.

\section{a) Data Processing}

The various processing steps involved in constructing total flux surface brightness maps and point-source filtered maps from the detector data streams are described in detail in the IRAS Explanatory Supplement (1985, hereafter IRAS Supple- 
ment) and the Pointed Observation Products Guide (Young et al. 1985, hereafter POG). Here, we describe the techniques used to remove residual photometric artifacts (intensity stripes) and background emission from the surface brightness maps.

\section{i) Detector Baseline Corrections}

Baseline variations in the detector data on the order of 10-15 times the data noise level resulted in discontinuities in the apparent intensity of the surface brightness maps orthogonal to the telescope scanning direction (e.g., Habing et al. 1984). This striping was corrected by comparing the data streams of adjacent wavelength band detectors of the detector array. The differences between adjacent spatial data samples of the paired detectors were histogrammed and the mode, or, if that failed, the median, of the differences was identified and used as an estimate of the offset between the detectors. This process was continued across the focal-plane detector array, producing a cross-scan offset profile for each band. The profiles were adjusted to zero mean and subtracted from the data. This technique also removed gross cross-scan field gradients in the data, along with the baseline offsets.

\section{ii) Background Removal}

A first estimate of the underlying background level in the surface brightness maps due to diffuse zodiacal and Galactic emission was taken to be the median of the map pixel values. This value was subtracted from the maps. Residual field gradients as large as 20 times the noise level remained in the maps. These were removed using one of two techniques. The first technique was similar to the de-stripping algorithm described above. The median differences of the pixel values in adjacent columns across the map were calculated, adjusted to zero mean, and removed. The process was then applied to the pixel rows of the map.

For maps with complicated backgrounds an alternate technique produced better results. A linear least-squares fit was computed for each pixel column of the map. The fits excluded the pixels of source emission with intensity values above a specified threshold. The parameters of the fitted lines, smoothed over the entire map, defined a surface that was removed.

The background removal processing resulted in maps in which the variation of the remaining background emission was typically less than $\sim 3$ times the map noise level over the entire field covered by the map. A few of the galaxies were embedded in patches of Galactic infrared "cirrus" emission (Low et al. 1984), and for these an estimate of the local background, taken to be the mean intensity within an outlying annular aperture centered on the galaxy image, was subtracted from the galaxy aperture measurements.

\section{b) Point-Source Filtered Maps}

The data were also processed to produce point-source filtered maps. In this processing, described in detail in the POG, a digital zero-sum bandpass filter centered on the IRAS point-source spatial frequency was applied to the spatially binned co-added detector data. This processing, which suppressed extended structure, resulted in a more sensitive measurement of the flux densities of unresolved sources.

\section{c) Total Flux Density Measurements}

The final maps were produced by co-adding the individual pointed observation or all-sky survey scan maps of the galaxy fields. The mean noise levels $(1 \sigma)$ of the $12,25,60$, and 100 $\mu \mathrm{m}$ surface brightness maps constructed from pointed observations are 4.6, 6.6, 3.8, and $7.2 \mathrm{mJy} \operatorname{arcmin}^{-2}$ (or 18, 26, 30 , and $110 \mathrm{mJy}$ per beam), respectively. The mean noise levels of the maps constructed from survey data are 11, 16, 8.5 , and $11 \mathrm{mJy} \operatorname{arcmin}^{-2}(45,64,68$, and $160 \mathrm{mJy}$ per beam), respectively.

Total flux densities were obtained from either the pointsource filtered maps or the surface brightness maps, depending on the spatial extent observed for the galaxy sources. For the sources for which a large infrared extent was not obvious, radial profiles of the galaxy surface brightness images along the IRAS in-scan and cross-scan directions were compared with the profiles of the star IRC +10011 , a representative $I R A S$ point source. The mean half-power width of the pointsource in-scan profile, measured from both co-added pointed observation and co-added survey data maps, is approximately 0.8 for the 12 and $25 \mu \mathrm{m}$ maps, 1'.6 for the $60 \mu \mathrm{m}$ maps, and $3^{\prime}$ for the $100 \mu \mathrm{m}$ maps. The cross-scan resolution (FWHM) is approximately $5^{\prime}$ for each wavelength map. (Note that in co-added maps composed of observations with a large range of scanning angles, unique in-scan and cross-scan directions do not exist. For these maps, the orientation of the profile cuts was based on the dominant scanning direction as determined by the shape of the sources. Typically, the component observations of a co-added map had scanning angles that coincided to within $\sim 20^{\circ}$.)

While the point-source filtered maps provide the best flux density measurements of unresolved sources, the point-source filtering can result in a significant underestimation of the integrated flux density of slightly extended sources. For this reason, the point-source filtered map measurements were taken as total flux densities only for sources with a pointlike surface brightness image at all detected wavelengths. If a source appeared extended in any one of the surface brightness wavelength maps, the total flux density at all detected wavelengths was obtained from the surface brightness maps. An exception was for faint 12 and $25 \mu \mathrm{m}$ detections with marginal surface brightness map flux density measurements $(<0.2 \mathrm{Jy})$. For these, the flux densities extracted from the more sensitive point-source filtered maps are reported.

Total flux densities were obtained from the surface brightness maps by summing the map pixel intensity values within the $25 \mathrm{mJy} \operatorname{arcmin}^{-2}(\sim 3-6 \sigma)$ brightness level contour of the galaxy image. If 3 times the map noise level was brighter than $25 \mathrm{mJy} \operatorname{arcmin}^{-2}$, the brighter $3 \sigma$ threshold was used. The selection of $25 \mathrm{mJy} \operatorname{arcmin}^{-2}$ as the brightness limit for determining the galaxy total infrared emission is somewhat arbitrary, since it is based on the overall sensitivity of the $I R A S$ data, along with the adequate removal of background emission from the maps using the techniques described above. However, the agreement between the optical size and the infrared $60 \mu \mathrm{m}$ size of the largest infrared extended galaxies to this brightness level (see $\S \mathrm{VI} b$ ) implies that the bulk of the emission is contained within the selected brightness limit. Furthermore, the small contribution to the total emission of the low-level infrared emission observed in the outer regions 
of the galaxies is consistent with the adopted surface brightness cutoff.

\section{d) Upper Limits and Uncertainties}

For galaxies not detected in a wavelength band, the reported upper limit is 3 times the noise level of the point-source filtered wavelength map for pointlike sources, or 3 times the noise level of the surface brightness wavelength map, integrated over the IRAS wavelength band beam, for extended sources. The surface brightness map $3 \sigma$ limits are reported for galaxies not detected in any band.

A more appropriate limit for a nondetection of an extended source may be the sum of the noise pixels of the surface brightness wavelength map over the observed infrared extent of the galaxy. Similarly, the nondetection of low-level diffuse emission at 12 and $25 \mu \mathrm{m}$ for a galaxy observed as more extended at 60 and $100 \mu \mathrm{m}$ could result in an underestimation of the "total" emission at the shorter wavelengths. Both possible underestimations assume an extended infrared emission model of galaxies in which the distribution of the diffuse emission at the various IRAS wavelengths is similar. The data analysis to determine the validity of this model has been deferred to Paper II, and the faint 12 and $25 \mu \mathrm{m}$ total flux density measurements and upper limits reported in this cata$\log$ for extended galaxies should be viewed with caution.

The photometric accuracy of these measurements is compromised by uncertainties in the frequency response of the detectors as a function of source strength and undersampling due to dead and noisy detectors. While the undersampling can cause large integrated flux density errors ( $\sim 25 \%$ or more) in the surface brightness maps for pointlike sources observed along tracks of the detector array containing the aberrant detectors, the uncertainties improve to $15 \%$ or better for sources observed either along the detector array prime track (i.e., targeted objects of pointed observations) or along a mixture of detector tracks (i.e., co-added survey data). For extended sources with half-power widths of $4^{\prime}$ or more (i.e., on the order of the cross-scan detector size) the estimated uncertainties are $\sim 15 \%$ (POG). The flux density uncertainties of point sources extracted from co-added pointed observation and survey data point-source filtered maps, determined by comparing the flux densities of standard stars to PSC values, are $\sim 10 \%$.

Co-added survey data surface brightness maps were constructed for a subset of the galaxies with pointed observations, including the LMC and the SMC. Except for the faintest 12 and $25 \mu \mathrm{m}$ sources ( $<2 \mathrm{Jy}$ ), the flux densities obtained from the lower sensitivity survey data maps were in agreement, within the uncertainties, with the values obtained from the pointed observation maps. The survey data map flux densities of the fainter sources were as much as 50\% lower than the pointed observation map measurements, depending on the depth of the survey coverage.

\section{THE CATALOG}

\section{a) Total Flux Densities}

Table 2 lists the "total" IRAS flux densities of the sample galaxies obtained from co-added maps and the "point-source" flux densities of these galaxies reported in the PSC. The column entries are described below.

Column (1)._-Galaxy name.

Column (2).-Observation type. "PO" denotes that the galaxy maps were constructed from pointed observation data; "SS" denotes that the maps were constructed from all-sky survey scan data.

Column (3). - Spatial extent code. A letter code has been assigned to indicate the degree of spatial extent evident in the IRAS surface brightness images of the galaxies and from which type of IRAS maps the galaxy total flux densities or upper limits were obtained. The code is given below.

E-The galaxy was observed as an extended source in one or more of the surface brightness wavelength maps; the total flux densities or upper limits were obtained from the surface brightness maps.

$\mathrm{P}$ - The galaxy was observed as an unresolved point source at all detected wavelengths; the total flux densities or upper limits were obtained from the point-source filtered maps.

$\mathrm{N}$ - The galaxy was not detected; the reported upper limits are 3 times the noise level of the surface brightness maps integrated over the IRAS beam.

Columns (4)-(7).-IRAS total flux density, $f_{\nu}$, at 12,25 , 60 , and $100 \mu \mathrm{m}$ in janskys $\left(10^{-26} \mathrm{~W} \mathrm{~m}^{-2} \mathrm{~Hz}^{-1}\right)$ measured from co-added maps, as described in $\S$ III $c$. The flux density of a faint extended source obtained from a point-source filtered map is followed by a "P." A colon following an entry denotes a larger flux density uncertainty because of poor background removal, the low sensitivity of a co-added survey data map, or for the reason listed in the notes to the table. (As in the PSC, flux densities have been calculated assuming an input energy distribution with $f_{v}$ proportional to $\nu^{-1}$.)

Column (8).-Designation of the positionally associated IRAS point-source detection listed in the PSC. For galaxies associated with multiple PSC entries (cf. Table IIIA.2 in the Cataloged Galaxies Observed in the IRAS Survey [1985, hereafter Extragalactic Catalog]), the entry listed is the detection nearest the galaxy optical center. PSC flux densities are not reported for the SMC and the LMC; both galaxies generated many PSC sources.

Columns (9)-(12)._IRAS "point-source" flux densities as listed in the PSC (Version 1.0). A colon following an entry denotes a moderate quality flux density as described in the IRAS Supplement (§ V.H.5).

Special comments for several of the galaxies are presented in the notes to the table.

\section{b) Global Emission Properties}

Table 3 reports color-corrected total flux densities at 12,25 , 60 , and $100 \mu \mathrm{m}$, along with the global emission of the galaxies at optical, near-infrared, and radio wavelengths compiled from the literature. The IRAS color corrections were calculated using a two-temperature thermal model fit to the measured flux densities or limits. The model is described below. The column entries of Table 3 are described below. 


\begin{tabular}{|c|c|c|c|c|c|c|c|c|c|c|c|}
\hline \multicolumn{12}{|c|}{$\begin{array}{c}\text { TABLE } 2 \\
\text { IRAS FLUX DENSITIES }\end{array}$} \\
\hline \multirow[b]{2}{*}{$\begin{array}{c}\text { Name } \\
(1)\end{array}$} & \multirow[b]{2}{*}{$\begin{array}{c}\mathrm{Obs}^{\mathrm{b}} \\
(2)\end{array}$} & \multirow[b]{2}{*}{$\begin{array}{c}\operatorname{Ext}^{c} \\
(3)\end{array}$} & \multicolumn{4}{|c|}{ Total Flux Density (Jy) } & \multirow[b]{2}{*}{$\begin{array}{c}\text { IRAS Name } \\
(8)\end{array}$} & \multicolumn{4}{|c|}{ PSC Flux Density (Jy) } \\
\hline & & & $\begin{array}{c}12 \mu \mathrm{m} \\
(4) \\
\end{array}$ & $\begin{array}{c}25 \mu \mathrm{m} \\
(5)\end{array}$ & $\begin{array}{c}60 \mu \mathrm{m} \\
(6)\end{array}$ & $\begin{array}{c}100 \mu \mathrm{m} \\
(7)\end{array}$ & & $\begin{array}{c}12 \mu \mathrm{m} \\
(9)\end{array}$ & $\begin{array}{c}25 \mu \mathrm{m} \\
(10)\end{array}$ & $\begin{array}{c}60 \mu \mathrm{m} \\
(11)\end{array}$ & $\begin{array}{c}100 \mu \mathrm{m} \\
(12)\end{array}$ \\
\hline N45 & SS & $\mathrm{E}$ & $<0.12$ & $<0.17$ & 1.62 & 4.99 & $00115-2327$ & $<0.25$ & $<0.25$ & $<0.45$ & 2.75 \\
\hline N55 & $\mathrm{PO}$ & $\mathrm{E}$ & 1.34 & 6.25 & 77.00 & 174.09 & $00125-3928$ & $0.56:$ & 1.77 & 30.96 & 73.33: \\
\hline N134 & SS & $\mathrm{E}$ & 1.77 & 2.67 & 17.23 & 61.18 & $00278-3331$ & 1.39 & 1.97 & 19.02 & 53.33 \\
\hline N147 & PO & $\mathrm{N}$ & $<0.05$ & $<0.06$ & $<0.08$ & $<0.36$ & & $\ldots$ & $\ldots$ & $\ldots$ & $\ldots$ \\
\hline N185 & PO & $\mathrm{P}$ & 0.04 & $<0.03$ & 0.31 & 1.93 & $00362+4803$ & $<0.29$ & $<0.25$ & $<0.44$ & 2.11 \\
\hline N205 & PO & $\mathrm{E}$ & $0.15 \mathrm{P}$ & $0.19 \mathrm{P}$ & 0.60 & 3.78 & $00376+4124$ & $<0.25$ & $<0.24$ & $<0.59$ & 3.10 \\
\hline M31 & $\mathrm{PO}$ & $\mathrm{E}$ & 163.23 & 107.71 & 536.18 & 2928.40 & $00400+4059$ & 1.15: & $0.75:$ & 7.53 & 14.37: \\
\hline $\mathrm{N} 247$ & SS & $\mathrm{E}$ & $<0.12$ & $<0.16$ & 7.93 & 27.32 & $00446-2101$ & $<0.94$ & $<0.25$ & $<0.78$ & 6.07 \\
\hline N253 & SS & $\mathrm{E}$ & 55.84 & 155.65 & 998.73 & 1861.67 & $00450-2533$ & 20.52 & 117.08 & 758.62 & 1044.83 \\
\hline SMC & $\mathrm{PO}^{(1)}$ & $\mathrm{E}$ & 67.03 & 270.18 & 6688.91 & 15021.93 & & $\ldots$ & $\ldots$ & $\ldots$ & $\ldots$ \\
\hline N300 & SS & $\mathrm{E}$ & $0.53:$ & $0.64:$ & 23.08 & 74.45 & $00523-3756$ & $<0.99$ & $<0.78$ & $<1.10$ & 8.43 \\
\hline I1613 & $\mathrm{PO}$ & $\mathrm{E}$ & $<0.06$ & $<0.14$ & 1.42 & 3.69 & $01025+0153$ & $<1.56$ & $<0.29$ & 1.01 & 2.64 \\
\hline M33 & $\mathrm{PO}$ & $\mathrm{E}$ & 32.69 & 40.26 & 419.65 & 1256.43 & $01310+3024$ & 0.49 & $<0.37$ & 9.93 & $<23.79$ \\
\hline N628 & SS & $\mathrm{E}$ & 2.07 & 1.90 & 20.86 & 65.64 & $01340+1532$ & $<0.25$ & $<0.40$ & 2.95 & 11.78 \\
\hline N660 & SS & $\mathrm{P}$ & 2.31 & 7.05 & 67.27 & 104.89 & $01403+1323$ & 2.02 & 7.12 & 64.99 & 102.38 \\
\hline N891 & PO & $\mathrm{E}$ & 5.66 & 7.78 & 61.10 & 198.63 & $02193+4207$ & 0.93 & 0.85 : & 34.08 & 146.28 \\
\hline N925 & $\mathrm{PO}$ & $\mathrm{E}$ & 0.26 & 0.66 & 7.65 & 26.68 & $02243+3321$ & $<0.25$ & $<0.25$ & 2.82 & 11.90 \\
\hline $\mathrm{N} 1023$ & SS & $\mathrm{P}$ & 0.16 & $<0.09$ & $<0.13$ & $<0.30$ & & $\ldots$ & $\ldots$ & $\ldots$ & $\ldots$ \\
\hline Fornax Sys. & SS & $\mathrm{N}$ & $<0.09$ & $<0.11$ & $<0.15$ & $<0.43$ & & $\ldots$ & $\ldots$ & $\ldots$ & $\ldots$ \\
\hline N1097 & $\mathrm{PO}$ & $\mathrm{E}$ & 2.88 & 7.70 & 46.73 & 116.34 & $02441-3029$ & 1.85 & 5.83 & 45.49 & 82.53 \\
\hline $\mathrm{N} 1291$ & $\mathrm{PO}$ & $\mathrm{E}$ & $0.18 \mathrm{P}$ & $0.17 \mathrm{P}$ & 1.76 & 10.13 & $03154-4117$ & $<0.28$ & $<0.24$ & 1.68 & 5.56 \\
\hline N1313 & $\mathrm{PO}$ & $\mathrm{E}$ & 0.95 & 3.49 & 35.97 & 92.00 & $03176-6640$ & $<0.25$ & 0.57 & 10.23 & 30.15 \\
\hline N1365 & $\mathrm{PO}$ & $\mathrm{E}$ & 4.42 & 13.07 & 84.20 & 185.40 & $03317-3618$ & 3.22 & 11.13 & 77.75 & 139.94 \\
\hline $\mathrm{I} 342$ & $\mathrm{PO}$ & $\mathrm{E}$ & 23.66 & 45.20 & 255.96 & 661.68 & $03419+6756$ & 3.80 & 18.76 & 85.15 & 125.97 \\
\hline N1448 & $\mathrm{PO}$ & $P$ & 0.89 & 1.08 & 9.92 & 34.07 & $03428-4448$ & 0.64 & 0.69 & 7.92 & 26.22 \\
\hline N1560 & $\mathrm{PO}$ & $\mathrm{E}$ & $<0.05$ & $<0.05$ & 2.15 & 5.32 & $04271+7146$ & $<0.25$ & $<0.25$ & 0.87 & 4.09 \\
\hline LMC & $\mathrm{PO}^{(1)}$ & $\mathrm{E}$ & 2781.90 & 7824.19 & 82917.00 & 184686.69 & & $\ldots$ & $\ldots$ & $\ldots$ & $\ldots$ \\
\hline N2403 & $\mathrm{PO}$ & $\mathrm{E}$ & 3.34 & 6.29 & 51.55 & 148.49 & $07321+6543$ & $<0.28$ & 1.30 & 11.46 & 54.65 \\
\hline N2683 & $\mathrm{PO}$ & $\mathrm{E}$ & 0.93 & 0.52 & 8.33 & 34.02 & $08495+3336$ & $<0.25$ & $<0.25$ & 4.79 & 24.02 \\
\hline N2841 & $\mathrm{PO}$ & $\mathrm{E}$ & 0.90 & 0.83 & 4.41 & 24.21 & $09185+5111$ & $<0.25$ & $<0.25$ & 2.41 & 13.51 \\
\hline $\mathrm{N} 2903$ & $\mathrm{PO}$ & $\mathrm{E}$ & 5.00 & 7.64 & 52.38 & 147.36 & $09293+2143$ & 0.86 & 2.33 & 27.93 & 102.64 \\
\hline $\mathrm{N} 2997^{\mathrm{d}}$ & $\mathrm{PO}$ & $\mathrm{E}$ & 3.13 & 5.06 & 32.28 & 85.14 & & $\ldots$ & $\ldots$ & $\ldots$ & $\ldots$ \\
\hline M81 & $\mathrm{PO}$ & $\mathrm{E}$ & 5.86 & 5.42 & 44.73 & 174.02 & $09514+6918$ & 0.67 & 0.75 & 6.88 & 25.21 \\
\hline M82 & $\mathrm{PO}$ & $\mathrm{E}$ & 66.61 & 285.03 & 1271.32 & 1351.09 & $09517+6954$ & 53.21 & 273.98 & 1167.76 & 1145.11 \\
\hline N3109d & $\mathrm{PO}$ & $\mathrm{E}$ & $<0.04$ & $0.07 \mathrm{P}$ & 3.41 & 7.97 & & $\ldots$ & $\ldots$ & $\ldots$ & $\ldots$ \\
\hline N3115 & SS & $\mathrm{P}$ & 0.19 & $<0.11$ & $<0.13$ & $<0.30$ & & $\ldots$ & $\ldots$ & $\ldots$ & $\ldots$ \\
\hline Leo I & $\mathrm{PO}$ & $\mathrm{N}$ & $<0.04$ & $<0.10$ & $<0.06$ & $<0.23$ & & $\ldots$ & $\ldots$ & $\ldots$ & $\ldots$ \\
\hline N3198 & $\mathrm{SS}$ & $\mathrm{E}$ & 0.34 & 0.61 & 6.46 & 17.69 & $10168+4548$ & $<0.25$ & 0.46 & 3.66 & 14.33 \\
\hline $\mathrm{I} 2574$ & $\mathrm{PO}$ & $\mathrm{E}$ & $<0.05$ & $0.08 \mathrm{P}$ & 2.41 & 10.62: & $10251+6843$ & $<0.30$ & $<0.25$ & 1.09 & 2.55 \\
\hline N3521 & $\mathrm{SS}$ & $\mathrm{E}$ & 4.91 & 4.36 & 44.02 & 124.80 & $11032+0014$ & $<0.98$ & $0.92:$ & 26.97 & 83.74 \\
\hline N3556 & SS & $\mathrm{E}$ & 2.25 & 4.09 & 32.19 & 80.77 & $11085+5556$ & 0.61 & 1.80 & 23.26 & 60.13 \\
\hline N3621 & $\mathrm{PO}$ & $\mathrm{E}$ & 3.47 & 5.09 & 29.62 & 90.12 & $11158-3232$ & $0.60:$ & 0.71 & 13.66 & 50.74 \\
\hline N3623 & SS & $\mathrm{E}$ & $<0.12$ & $<0.21$ & 2.99 & 15.27 & $11163+1322$ & $<0.25$ & $<0.34$ & 2.03 & 12.92 \\
\hline N3627 & SS & $\mathrm{E}$ & 4.17 & 7.72 & 56.31 & 144.96 & $11176+1315$ & 0.72 : & 1.37: & 33.69: & 104.20 \\
\hline N3628 & SS & $\mathrm{E}$ & 3.08 & 5.30 & 48.51 & 122.17 & $11176+1351$ & 2.61 & 4.67: & 48.03 & 101.73 \\
\hline
\end{tabular}




\begin{tabular}{|c|c|c|c|c|c|c|c|c|c|c|c|}
\hline \multicolumn{12}{|c|}{ TABLE 2-Continued } \\
\hline \multirow[b]{2}{*}{$\begin{array}{c}\text { Name } \\
(1)\end{array}$} & \multirow[b]{2}{*}{$\begin{array}{c}\mathrm{Obs}^{\mathrm{b}} \\
(2)\end{array}$} & \multirow[b]{2}{*}{$\begin{array}{c}E^{2} t^{c} \\
(3)\end{array}$} & \multicolumn{4}{|c|}{ Total Flux Density (Jy) } & \multirow[b]{2}{*}{$\begin{array}{c}\text { IRAS Name } \\
(8)\end{array}$} & \multicolumn{4}{|c|}{ PSC Flux Density (Jy) } \\
\hline & & & $\begin{array}{c}12 \mu \mathrm{m} \\
(4) \\
\end{array}$ & $\begin{array}{c}25 \mu \mathrm{m} \\
(5)\end{array}$ & $\begin{array}{c}60 \mu \mathrm{m} \\
(6)\end{array}$ & $\begin{array}{c}100 \mu \mathrm{m} \\
(7)\end{array}$ & & $\begin{array}{c}12 \mu \mathrm{m} \\
(9)\end{array}$ & $\begin{array}{c}25 \mu \mathrm{m} \\
(10)\end{array}$ & $\begin{array}{c}60 \mu \mathrm{m} \\
(11) \\
\end{array}$ & $\begin{array}{c}100 \mu \mathrm{m} \\
(12)\end{array}$ \\
\hline N3718 & SS & $\mathrm{P}$ & 0.15 & 0.11 & 0.76 & 2.52 & $11298+5320$ & $<0.36$ & $<0.62$ & $<0.81$ & 2.81 \\
\hline N4192 & SS & $\mathrm{E}$ & $0.65:$ & $0.36:$ & 7.19 & 23.18 & $12112+1510$ & $<0.33$ & $<0.45$ & 5.02 & 18.38 \\
\hline $\mathrm{N} 4216$ & SS & $\mathrm{E}$ & $<0.12$ & $<0.20$ & 2.27 & 12.79 & $12133+1325$ & $<0.34$ & $<0.31$ & $<0.93$ & 7.42 \\
\hline $\mathrm{N} 4236$ & $\mathrm{PO}$ & $\mathrm{E}$ & $0.11 \mathrm{P}$ & $0.57 \mathrm{P}$ & 3.98 & 10.02 & $12140+6947$ & $<0.25$ & 0.29: & 1.60 & 3.93 \\
\hline $\mathrm{N} 4244$ & $\mathrm{PO}$ & $\mathrm{E}$ & $<0.05$ & $<0.07$ & 4.20 & 16.06 & $12150+3804$ & $<0.36$ & $<0.25$ & $<0.45$ & 2.94 \\
\hline $\mathrm{N} 4258^{\mathrm{d}}$ & $\mathrm{PO}$ & $\mathrm{E}$ & 2.25 & 2.81 & 21.60 & 78.39 & & $\ldots$ & $\ldots$ & $\ldots$ & $\ldots$ \\
\hline $\mathrm{N} 4395$ & SS & $\mathrm{E}$ & $<0.11$ & $0.17 \mathrm{P}$ & 4.21 & 12.90 & $12233+3348$ & $<0.25$ & $<0.25$ & 2.07 & 5.68 \\
\hline $\mathrm{N} 4438$ & SS & $\mathrm{P}$ & 0.17 & $<0.15$ & 4.28 & 12.05 & $12252+1317$ & $<0.27$ & $<0.27$ & 4.00 & 10.44 \\
\hline $\mathrm{N} 4472$ & SS & $\mathrm{N}$ & $<0.13$ & $<0.21$ & $<0.19$ & $<0.48$ & & $\ldots$ & $\ldots$ & $\ldots$ & $\ldots$ \\
\hline N4517 & SS & $\mathrm{E}$ & $0.61:$ & $0.53:$ & 6.92 & 20.20 & $12301+0023$ & $<0.31$ & 0.56 & 3.91 & 14.41 \\
\hline N4559 & SS & $\mathrm{E}$ & 0.49: & $0.73:$ & 9.69 & 27.05 & $12334+2814$ & $<0.40$ & 0.43 & 5.25 & 17.45 \\
\hline N4565 & $\mathrm{PO}$ & $\mathrm{E}$ & 1.53 & 1.70 & 9.83 & 47.23 & $12338+2615$ & $<0.90$ & $0.58:$ & 5.94 & 24.69: \\
\hline N4569 & SS & $E$ & $0.75:$ & 1.28: & 9.19 & 27.33 & $12343+1326$ & $<0.35$ & 0.89 & 7.08 & 22.72 \\
\hline N4594 & SS & $\mathrm{E}$ & $0.74:$ & $0.50:$ & 4.26 & 22.86 & $12373-1120$ & $<0.57$ & $0.43:$ & 3.12 & 11.72 \\
\hline N4631 & $\mathrm{PO}$ & $\mathrm{E}$ & 5.48 & 9.65 & 82.90 & 208.66 & $12396+3249$ & 1.82 & 3.01 & 51.15 & 118.60 \\
\hline N4656 & $\mathrm{PO}$ & $\mathrm{E}$ & $0.10 \mathrm{P}$ & $0.30 \mathrm{P}$ & 5.90 & 11.46 & $12415+3226$ & $<0.69$ & $<0.34$ & 2.17 & 6.08 \\
\hline N4725 & SS & $\mathrm{E}$ & $0.32:$ & $0.20 \mathrm{P}$ & 4.18 & 20.79 & $12480+2547$ & $<0.59$ & $<0.25$ & 0.75 & $<7.00$ \\
\hline N4736 & $\mathrm{PO}$ & $\mathrm{E}$ & 4.77 & 6.83 & 62.41 & 135.34 & $12485+4123$ & 2.79 & 3.50 & 55.70 & 103.78 \\
\hline N4762 & SS & $\mathrm{P}$ & 0.14 & $<0.15$ & $<0.15$ & $<0.35$ & & $\ldots$ & $\ldots$ & $\ldots$ & $\ldots$ \\
\hline N4826 & SS & $\mathrm{P}$ & 1.71 & 2.00 & 33.86 & 77.38 & $12542+2157$ & 1.83 & 2.57 & 36.89 & 74.47: \\
\hline N4945 & $\mathrm{PO}$ & $\mathrm{E}$ & 23.65 & 43.28 & 588.11 & 1415.50 & $13025-4911$ & 3.65 & 14.32 & 388.05 & 684.01 \\
\hline N5033 & SS & $\mathrm{E}$ & 1.38 & 1.77 & 17.20 & 51.05 & $13111+3651$ & 0.78 & 1.06 & 13.08 & 43.55 \\
\hline N5055 & $\mathrm{PO}$ & $\mathrm{E}$ & 5.56 & 7.00 & 40.02 & 157.74 & $13135+4217$ & 1.21: & 1.15 & 27.64 & 99.84 \\
\hline N5102 & SS & $\mathrm{E}$ & $<0.10$ & $<0.14$ & 0.82 & 2.73 & $13191-3622$ & $<0.25$ & $<0.25$ & 0.82 & 2.55 \\
\hline N5128 & $\mathrm{PO}$ & $\mathrm{E}$ & 23.03 & 30.74 & 217.57 & 501.20 & $13225-4245$ & 11.20 & 15.07 & 171.12 & 335.73 \\
\hline N5170 & SS & $\mathrm{P}$ & 0.15 & $<0.16$ & 1.08 & 4.84 & $13271-1742$ & $<0.25$ & $<0.31$ & 1.02 & 3.59 \\
\hline M51 & $\mathrm{PO}$ & $\mathrm{E}$ & 11.02: & 17.47: & 108.68: & 292.08: & $13277+4727$ & 1.37 & 2.40 & 31.68 & 121.42 \\
\hline M83 & $\mathrm{PO}^{(2)}$ & $E$ & 26.28 & 47.72 & 266.03 & 638.63 & $13341-2936$ & 4.72 & 19.61 & 103.25 & 212.05 \\
\hline M101 & $\mathrm{PO}$ & $\mathrm{E}$ & 6.20 & 11.78 & 88.04 & 252.84 & $14013+5435$ & $<0.52$ & 0.35 & 3.83 & 29.90 \\
\hline $\mathrm{U} \mathrm{Mi}$ & $\mathrm{PO}$ & $\mathrm{N}$ & $<0.04$ & $<0.05$ & $<0.07$ & $<0.24$ & & $\ldots$ & $\ldots$ & $\ldots$ & $\ldots$ \\
\hline N5907 & $\mathrm{PO}$ & $\mathrm{E}$ & 1.22 & 1.35 & 8.78 & 45.76 & $15146+5629$ & 0.90 & 0.94 & 9.89 & 35.13 \\
\hline Draco Sys. & $\mathrm{PO}$ & $\mathrm{N}$ & $<0.06$ & $<0.08$ & $<0.10$ & $<0.30$ & $17185+5756$ & $<0.25$ & $<0.25$ & 0.65 & 1.11 \\
\hline $\mathrm{N} 6744$ & $\mathrm{PO}$ & $\mathrm{E}$ & 2.86 & 4.18 & 22.21 & 85.80 & $19047-6357$ & $<0.25$ & $<0.25$ & 2.02 & 16.94 \\
\hline N6822 & $\mathrm{PO}$ & $\mathrm{E}$ & 0.25 & 2.46 & 47.63: & 95.42: & $19421-1455$ & $<0.83$ & $<0.33$ & 1.91 & $<11.44$ \\
\hline N6946 & $\mathrm{PO}$ & $\mathrm{E}$ & 12.13 & 21.18 & 136.69 & 344.37 & $20338+5958$ & 2.17 & 6.56 & 52.07 & 126.37 \\
\hline I5201 & SS & $\mathrm{E}$ & $0.15 \mathrm{P}$ & $<0.10$ & 1.42 & 3.31 & $22179-4617$ & $<0.25$ & $<0.25$ & 0.41: & 3.11 \\
\hline N7331 & SS & $\mathrm{E}$ & 3.36 & 4.20 & 35.29 & 115.07 & $22347+3409$ & $<0.46$ & $<0.25$ & 19.02: & 80.89 \\
\hline N7640 & SS & $\mathrm{E}$ & $0.16 \mathrm{P}$ & $<0.13$ & 3.70 & 11.45 & $23197+4034$ & $<0.25$ & $<0.24$ & 1.70 & 7.28 \\
\hline N7793 & $\mathrm{PO}^{(2)}$ & $E$ & 1.54 & 2.09 & 19.62 & 56.34 & $23552-3252$ & $<0.30$ & $<0.39$ & 5.59 & 32.54 \\
\hline WLM & SS & $\mathrm{E}$ & $<0.12$ & $<0.20$ & 0.32 & 1.04 & & $\ldots$ & $\ldots$ & $\ldots$ & $\ldots$ \\
\hline
\end{tabular}

${ }^{a}$ Flux densities have been calculated assuming an input energy distribution proportional to $\nu^{-1}$. Version 1.0 PSC values are reported.

${ }^{b}$ Observation type: Maps were constructed from pointed observations (PO) or all-sky survey scans (SS). (1) LMC and SMC data are mean measurements from two sets of PO maps and one set of SS maps for each galaxy. (2) Maps constructed from single PO data.

${ }^{c}$ Extent code key: E-galaxy was observed as an extended source; the total flux densities were obtained from surface brightness maps. Entries followed by $\mathrm{P}$ were measured from point-source filtered maps. P-galaxy was observed as an "unresolved" source; the total flux densities were obtained from point-source filtered maps. N-galaxy was not detected.

${ }^{d}$ N2997, N3109, and N4258 were not covered in the all-sky survey but were the targets of pointed observations.

\section{REMARKS TO TABLE 2}

N45.- Nearby background galaxies detected at $3 \sigma$ level in the 60 and $100 \mu \mathrm{m}$ maps.

N147. - Heavy cirrus in field.

N185. - Heavy cirrus in field; $100 \mu \mathrm{m}$ source possibly confused due to cirrus.

$S M C$. - Integrated emission within $3^{\circ}$ of $0051.00-7306.0$; the 12 and $25 \mu \mathrm{m}$ emission of the foreground source NGC 104 has been removed (cf. Schwering and Israel 1987).

I1613. - Prominent emission from cluster of $\mathrm{H}$ II regions northeast of the nucleus and low-level emission coincident with optical knots southeast of the nucleus. 


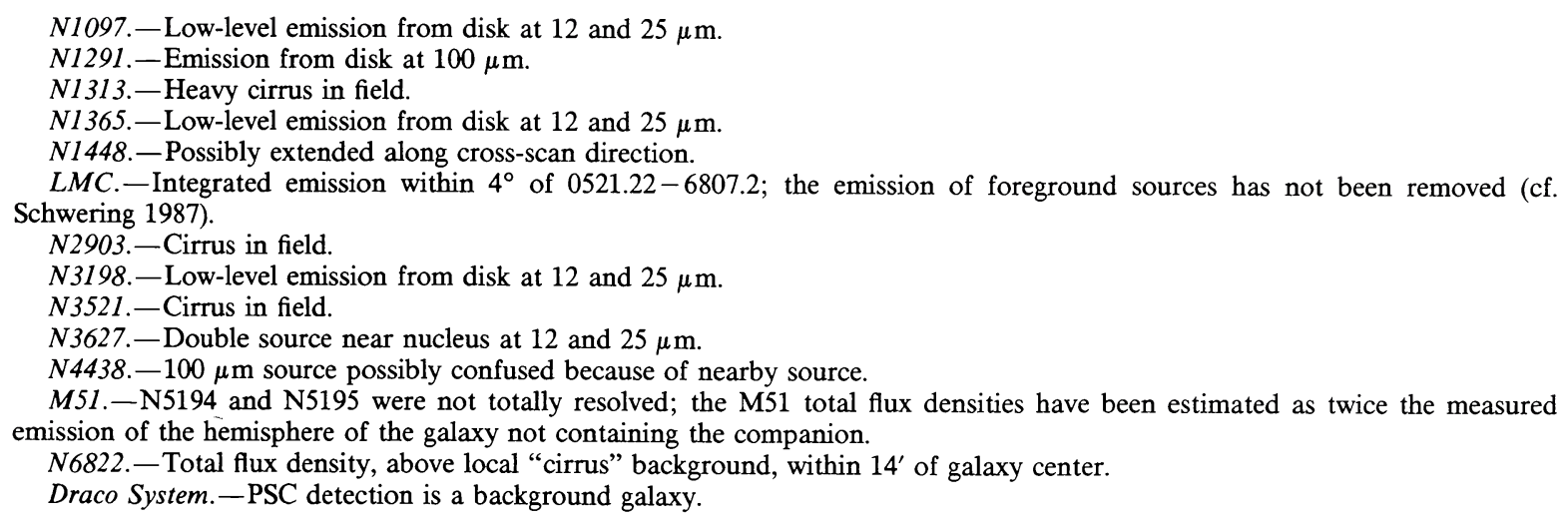

Column (1).-Galaxy name.

Column (2). $-B_{T}^{0}$, the total blue magnitude corrected for Galactic and internal extinction. The RC2 magnitudes have been recalculated using the correction formulae of $\mathrm{RC} 2$ and the Galactic extinction estimates of Burstein and Heiles (1984).

Columns $(3)-(4) .-(B-V)_{T}^{0}$ and $(U-B)_{T}^{0}$, integrated color indices corrected for Galactic and internal extinction. The RC2 colors have been recalculated using the correction formulae of RC2 and the Galactic extinction estimates of Burstein and Heiles (1984).

Column (5).-Magnitude in the near-infrared $H$ band (1.65 $\mu \mathrm{m})$ as listed in Aaronson et al. (1982) and the sources cited therein. The magnitudes were corrected for Galactic extinction and refer to an aperture-to-diameter ratio $\log (A / D)=$ -0.5 .

Columns (6)-(9).-IRAS color-corrected total flux densities at $12,25,60$, and $100 \mu \mathrm{m}$.

Column (10).- Integrated flux density in H I $21 \mathrm{~cm}$ line emission as listed in Aaronson et al. (1982), Huchtmeier et al. (1983), and the sources cited therein.

Column (11). - Mean integrated radio continuum flux density at $1400 \mathrm{MHz}$ as listed in RC2, Hummel (1980), and the sources cited therein.

Column (12). - Integrated flux density in CO from Sanders and Young (1986) and Verter (1985).

\section{c) Integrated Infrared Properties}

Since a single-temperature model cannot generally fit $I R A S$ galaxy data (Extragalactic Catalog), a two-temperature model was used to estimate the total flux of the mid- and far-infrared emission of the galaxies. Both the warm and the cold component energy distributions of the model were Planck curves with an emissivity law proportional to $\lambda^{-1}$. The two distributions, convolved with the spectral response of the four IRAS bandpasses (IRAS Supplement, Table II.C.5), were iteratively fitted to the measured flux densities or limits reported in Table 2. While measurable flux densities at both 60 and 100 $\mu \mathrm{m}$ were requirements for using the model, if only upper limits at 12 and $25 \mu \mathrm{m}$ were available these values were used. For an upper limit, the model fit was to one-half the $3 \sigma$ limit value with an uncertainty set equal to one-half the limit value. An uncertainty of $15 \%$ was assigned to measured flux densities. The mean correction was $14 \%,-15 \%, 4 \%$, and $4 \%$ for the $12,25,60$, and $100 \mu \mathrm{m}$ flux densities, respectively.
The total fluxes of the warm and cold components of the model, and the total flux of the combined components, were obtained by integrating the two final fitted curves over all wavelengths. Warm and cold component dust temperatures were also derived from the fits. ${ }^{5}$

The model results are reported in Table 4 . The fluxes were converted to luminosities using the distances listed in Table 1. The column entries are the following:

Column (1).- - Galaxy name.

Column (2). $-I R_{T}$, the total flux $\left(\mathrm{W} \mathrm{m}^{-2}\right)$ of the combined warm and cold component distributions of the two-temperature model.

Column (3). $-L\left(I R_{T}\right), I R_{T}$ converted to luminosity in solar units.

Column (4). $-I R_{C} / I R_{T} \equiv f_{C, T}$, the ratio of the total flux of the cold component distribution of the two-temperature model to the total flux of the combined distributions of the model.

Column (5). $-F_{\mathrm{IR}}$, the far-infrared flux $\left(\mathrm{W} \mathrm{m} \mathrm{m}^{-2}\right)$, an estimate of the total flux between 42.5 and $122.5 \mu \mathrm{m}$, defined in the Extragalactic Catalog as

$$
F_{\text {IR }}=1.26\left(2.58 f_{\nu}(60)+f_{\nu}(100)\right) \times 10^{-14},
$$

where $f_{\nu}(60)$ and $f_{\nu}(100)$ are the flux densities at the two far-infrared wavelengths as reported in Table 2 (see also Helou et al. 1987).

Column (6). $-L\left(F_{\mathrm{IR}}\right), F_{\mathrm{IR}}$ converted to luminosity in solar units.

Column (7). $-F_{\mathrm{IR}} / I R_{C} \equiv f_{\mathrm{FIR}, C}$, the ratio of the 42.5-122.5 $\mu \mathrm{m}$ flux to the total flux of the cold component distribution of the two-temperature model.

\footnotetext{
${ }^{5}$ While the two-temperature thermal model provides a better fit to the IRAS flux densities of galaxies, one should be cautious when applying physical interpretations to the total fluxes and dust temperatures derived from this simplified model. The true energy distribution of galaxies at mid- and far-infrared wavelengths is poorly understood (e.g., the relative contribution at the IRAS wavelengths of nonequilibrium emission from small dust grains). The derived quantities of the model are useful for statistical analyses intercomparing the infrared properties of the sample galaxies.

Note that the color-correction, total flux, and infrared temperature derivations may be affected by the sensitivity of the 12 and $25 \mu \mathrm{m}$ wavelength bands. As discussed in $\S \mathrm{III} d$, the reported 12 and $25 \mu \mathrm{m}$ flux densities (or limits) of faint extended sources may underestimate the total emission by excluding the contribution of low-level diffuse emission of the galaxy disk.
} 
Column (8). $-I R_{T} / F_{B}{ }^{*} \equiv f_{T, B}$, the ratio of the total flux of the combined distributions of the two-temperature model to the blue-light flux corrected for Galactic and internal reddening. Here, the blue-light flux is defined as $F_{B}{ }^{*}=\nu f_{\nu}$ at 0.44 $\mu \mathrm{m}$ (de Jong et al. 1984; Soifer et al. 1984) and differs from the commonly used "in-band" blue-light flux.

Column (9). $-F_{\mathrm{IR}} / F_{B}{ }^{*} \equiv f_{\mathrm{FIR}, B}$, the ratio of the 42.5-122.5 $\mu \mathrm{m}$ flux to the blue-light flux.
Column (10). $-T_{W}$, the dust temperature of the warm component. A colon following an entry means that the 12 or 25 $\mu \mathrm{m}$ flux density was an upper limit or both values were upper limits.

Column (11). $-T_{C}$, the dust temperature of the cold component.

The last three lines of the table list the median, the mean, and the standard deviation from the mean of the column

TABLE 3

GLOBAL EMISSION PROPERTIES

\begin{tabular}{|c|c|c|c|c|c|c|c|c|c|c|c|}
\hline \multirow[b]{2}{*}{$\begin{array}{c}\text { Name } \\
(1)\end{array}$} & \multirow[b]{2}{*}{$\begin{array}{l}B_{T}^{o} \\
(2) \\
\end{array}$} & \multirow[b]{2}{*}{$\begin{array}{c}(B-V)_{0} \\
(3)\end{array}$} & \multirow[b]{2}{*}{$\begin{array}{c}(U-B)_{\circ} \\
(4)\end{array}$} & \multirow[b]{2}{*}{$\begin{array}{c}1.65 \mu \mathrm{m} \\
(5)\end{array}$} & \multicolumn{4}{|c|}{ Color-corrected (Jy) } & \multirow[b]{2}{*}{$\begin{array}{c}\mathrm{HI} \\
(10)\end{array}$} & \multirow[b]{2}{*}{$\begin{array}{c}21 \mathrm{~cm} \\
(11)\end{array}$} & \multirow[b]{2}{*}{$\begin{array}{l}\mathrm{CO} \\
(12) \\
\end{array}$} \\
\hline & & & & & $\begin{array}{c}12 \mu \mathrm{m} \\
(6) \\
\end{array}$ & $\begin{array}{c}25 \mu \mathrm{m} \\
(7) \\
\end{array}$ & $\begin{array}{c}60 \mu \mathrm{m} \\
(8)\end{array}$ & $\begin{array}{c}100 \mu \mathrm{m} \\
(9)\end{array}$ & & & \\
\hline N45 & 10.93 & 0.65 & -0.07 & 9.65 & $\ldots$ & $\cdots$ & 1.71 & 5.21 & 236 & $\cdots$ & $\ldots$ \\
\hline N55 & 7.27 & $\ldots$ & $\ldots$ & $\ldots$ & 1.65 & 6.06 & 83.84 & 179.82 & $2450^{(3)}$ & $700^{(4)}$ & $\ldots$ \\
\hline $\mathrm{N} 134$ & 10.59 & 0.79 & 0.20 & 7.58 & 2.06 & 2.26 & 17.49 & 64.11 & 154 & $\ldots$ & $\ldots$ \\
\hline $\mathrm{N} 147$ & $9.69^{(1)}$ & 0.78 & $\ldots$ & $\ldots$ & $\ldots$ & $\ldots$ & $\ldots$ & $\ldots$ & $\ldots$ & $<50$ & $\ldots$ \\
\hline N185 & 9.29 & 0.72 & $\ldots$ & $\ldots$ & 0.03 & $\ldots$ & 0.28 & 2.03 & $36^{(3)}$ & $<50$ & $\ldots$ \\
\hline $\mathrm{N} 205$ & 8.71 & 0.81 & $\ldots$ & $\ldots$ & 0.17 & 0.16 & 0.52 & 3.97 & $\ldots$ & $<50$ & $\ldots$ \\
\hline M31 & 3.68 & 0.76 & 0.36 & 0.91 & 164.33 & 82.42 & 489.85 & 3080.75 & $\ldots$ & 8000 & $\ldots$ \\
\hline N247 & 8.99 & 0.55 & $\ldots$ & 7.69 & $\ldots$ & $\ldots$ & 8.23 & 28.60 & 740 & $<50$ & $\ldots$ \\
\hline N253 & 7.61 & 0.87 & $\ldots$ & 4.74 & 68.34 & 142.52 & 1095.99 & 1906.87 & $\ldots$ & 5500 & 23000 \\
\hline SMC & 2.39 & 0.41 & $\ldots$ & $\ldots$ & 82.58 & 255.73 & 7337.85 & 15505.51 & $\ldots$ & $67600^{(4)}$ & $\ldots$ \\
\hline N300 & 8.50 & $\ldots$ & $\ldots$ & $\ldots$ & 0.60 & 0.53 & 24.20 & 77.81 & $2010^{(3)}$ & $\ldots$ & $\ldots$ \\
\hline $\mathrm{I} 1613$ & 9.94 & 0.59 & $\ldots$ & $\ldots$ & $\ldots$ & $\ldots$ & 1.53 & 3.83 & $500^{(3)}$ & $<50$ & $\ldots$ \\
\hline M33 & 5.92 & 0.47 & -0.16 & 4.38 & 37.03 & 33.29 & 442.56 & 1310.68 & $\ldots$ & 3100 & $\ldots$ \\
\hline N628 & 9.60 & 0.55 & $\ldots$ & $\ldots$ & 2.23 & 1.51 & 21.85 & 68.57 & $650^{(3)}$ & 200 & $\ldots$ \\
\hline N660 & 11.08 & 0.74 & $\ldots$ & $\ldots$ & 2.83 & 6.53 & 74.66 & 106.39 & $150^{(3)}$ & 390 & $\ldots$ \\
\hline N891 & 10.05 & 0.73 & 0.09 & 6.84 & 6.52 & 6.53 & 63.30 & 207.70 & 225 & 770 & 6800 \\
\hline N925 & 10.17 & 0.49 & $\ldots$ & 8.74 & 0.32 & 0.60 & 7.83 & 27.94 & 307 & 65 & $\ldots$ \\
\hline N1023 & 9.86 & 0.92 & 0.47 & $\ldots$ & $\ldots$ & $\ldots$ & $\ldots$ & $\ldots$ & $45^{(3)}$ & $<50$ & $\ldots$ \\
\hline Fornax Sys. & 9.02 & $\ldots$ & $\ldots$ & $\ldots$ & $\ldots$ & $\ldots$ & $\ldots$ & $\ldots$ & $\ldots$ & $\ldots$ & $\ldots$ \\
\hline N1097 & 10.05 & 0.96 & $\cdots$ & $\cdots$ & 3.52 & 7.02 & 49.94 & 120.68 & $137^{(3)}$ & $540^{(4)}$ & $\cdots$ \\
\hline N1291 & 9.37 & 0.92 & 0.43 & $\ldots$ & 0.20 & 0.14 & 1.60 & 10.66 & $72^{(3)}$ & $200^{(4)}$ & $\ldots$ \\
\hline N1313 & 9.24 & $\ldots$ & $\ldots$ & $\ldots$ & 1.17 & 3.30 & 38.59 & 95.49 & $440^{(3)}$ & $\ldots$ & $\ldots$ \\
\hline N1365 & 9.93 & 0.57 & 0.02 & 7.20 & 5.42 & 12.05 & 91.22 & 191.37 & 159 & $616^{(4)}$ & $\ldots$ \\
\hline $\mathrm{I} 342$ & 7.75 & $\ldots$ & $\ldots$ & $\ldots$ & 28.27 & 39.53 & 272.65 & 687.25 & $4908^{(3)}$ & $2500^{(4)}$ & 35000 \\
\hline N1448 & 10.77 & $\ldots$ & $\ldots$ & 8.38 & 1.01 & 0.89 & 10.20 & 35.67 & 134 & $\ldots$ & $\ldots$ \\
\hline N1560 & 11.07 & 0.45 & $\ldots$ & 9.31 & $\ldots$ & $\ldots$ & 2.35 & 5.51 & 191 & $<50^{(4)}$ & $\ldots$ \\
\hline LMC & 0.31 & 0.47 & $\ldots$ & $\ldots$ & 3405.97 & 7157.10 & 90480.32 & 190632.61 & $\ldots$ & $616600^{(4)}$ & $\ldots$ \\
\hline $\mathrm{N} 2403$ & 8.52 & 0.42 & $\ldots$ & 6.45 & 3.99 & 5.49 & 54.43 & 154.75 & 1459 & 300 & $\ldots$ \\
\hline N2683 & 10.01 & 0.77 & 0.17 & 6.96 & 0.90 & 0.39 & 8.31 & 35.73 & 85 & 90 & $\ldots$ \\
\hline N2841 & 9.83 & 0.79 & 0.35 & 6.90 & 0.97 & 0.66 & 4.02 & 25.47 & 190 & 95 & $\ldots$ \\
\hline N2903 & 9.20 & 0.57 & 0.00 & 6.56 & 5.83 & 6.50 & 55.42 & 153.46 & 187 & 400 & $4900^{(5)}$ \\
\hline N2997 & 10.00 & $\ldots$ & $\ldots$ & $\ldots$ & 3.68 & 4.33 & 34.41 & 88.48 & $173^{(3)}$ & $708^{(4)}$ & $\ldots$ \\
\hline M81 & 7.39 & 0.85 & $\ldots$ & 4.38 & 6.33 & 4.32 & 44.82 & 182.65 & 266 & 380 & $\ldots$ \\
\hline M82 & 8.89 & 0.78 & $\ldots$ & $\ldots$ & 82.06 & 278.17 & 1375.27 & 1340.50 & $700^{(3)}$ & 8000 & 42000 \\
\hline N3109 & $9.69^{(1)}$ & $\ldots$ & $\ldots$ & $\ldots$ & $\ldots$ & 0.07 & 3.74 & 8.24 & $1280^{(3)}$ & 210 & $\ldots$ \\
\hline N3115 & 9.86 & 0.91 & 0.53 & $\ldots$ & $\ldots$ & $\ldots$ & $\ldots$ & $\ldots$ & $\ldots$ & $<50$ & $\ldots$ \\
\hline Leo I & 11.02 & 0.95 & $\ldots$ & $\ldots$ & $\ldots$ & $\ldots$ & $\ldots$ & $\ldots$ & $\ldots$ & $\ldots$ & $\ldots$ \\
\hline N3198 & 10.66 & 0.47 & $\ldots$ & 8.71 & 0.40 & 0.53 & 6.90 & 18.40 & 206 & $<100$ & $\ldots$ \\
\hline $\mathrm{I} 2574$ & 10.68 & 0.39 & $\ldots$ & 10.07 & $\ldots$ & 0.07 & 2.36 & 11.16 & 499 & $<70$ & $\ldots$ \\
\hline N3521 & 9.41 & 0.77 & $\ldots$ & 6.51 & 5.25 & 3.46 & 46.86 & 129.96 & 282 & 430 & $\ldots$ \\
\hline N3556 & 10.23 & 0.51 & -0.08 & $\ldots$ & 2.67 & 3.55 & 34.63 & 83.78 & $144^{(3)}$ & 293 & $\ldots$ \\
\hline N3621 & $9.48^{(1)}$ & $\ldots$ & $\ldots$ & 7.40 & 4.03 & 4.31 & 30.88 & 94.08 & 668 & $420^{(4)}$ & $\ldots$ \\
\hline N3623 & 9.74 & 0.80 & 0.32 & 6.76 & $\ldots$ & $\ldots$ & 2.82 & 16.07 & 26 & $<50$ & $\ldots$ \\
\hline N3627 & 9.40 & 0.63 & 0.17 & 6.70 & 4.97 & 6.72 & 60.30 & 150.51 & 61 & 500 & 8300 \\
\hline N3628 & 9.64 & 0.68 & $\ldots$ & 6.97 & 3.64 & 4.57 & 52.27 & 126.72 & 276 & 512 & 10000 \\
\hline
\end{tabular}


TABLE 3-Continued

\begin{tabular}{|c|c|c|c|c|c|c|c|c|c|c|c|}
\hline \multirow[b]{2}{*}{$\begin{array}{c}\text { Name } \\
(1)\end{array}$} & \multirow[b]{2}{*}{$\begin{array}{l}B_{T}^{o} \\
(2) \\
\end{array}$} & \multirow[b]{2}{*}{$\begin{array}{c}(B-V) \text { 。 } \\
(3)\end{array}$} & \multirow[b]{2}{*}{$\begin{array}{c}(U-B)_{\circ} \\
(4)\end{array}$} & \multirow[b]{2}{*}{$\begin{array}{c}1.65 \mu \mathrm{m} \\
(5)\end{array}$} & \multicolumn{4}{|c|}{ Color-corrected (Jy) } & \multirow[b]{2}{*}{$\begin{array}{c}\mathrm{HI} \\
(10)\end{array}$} & \multirow[b]{2}{*}{$\begin{array}{c}21 \mathrm{~cm} \\
(11)\end{array}$} & \multirow[b]{2}{*}{$\begin{array}{l}\mathrm{CO} \\
(12)\end{array}$} \\
\hline & & & & & $\begin{array}{c}12 \mu \mathrm{m} \\
(6)\end{array}$ & $\begin{array}{c}25 \mu \mathrm{m} \\
(7)\end{array}$ & $\begin{array}{c}60 \mu \mathrm{m} \\
(8)\end{array}$ & $\begin{array}{c}100 \mu \mathrm{m} \\
(9)\end{array}$ & & & \\
\hline N3718 & 11.02 & 0.68 & $\ldots$ & $\ldots$ & 0.15 & 0.09 & 0.79 & 2.64 & $\ldots$ & $<50$ & $\ldots$ \\
\hline N4192 & 10.34 & 0.67 & $\ldots$ & 7.77 & 0.63 & 0.27 & 7.53 & 24.23 & 83 & 90 & $\ldots$ \\
\hline N4216 & 10.43 & 0.88 & 0.43 & $\ldots$ & $\ldots$ & $\ldots$ & 2.08 & 13.46 & $23^{(3)}$ & $<50$ & $\cdots$ \\
\hline $\mathrm{N} 4236$ & 9.55 & 0.30 & $\ldots$ & 9.08 & 0.13 & 0.56 & 4.23 & 10.40 & 476 & $<50$ & $\ldots$ \\
\hline N4244 & 9.85 & 0.29 & $\ldots$ & 8.75 & $\ldots$ & $\ldots$ & 4.27 & 16.85 & 418 & $<50$ & $\cdots$ \\
\hline N4258 & 8.66 & 0.61 & $\ldots$ & 6.11 & 2.56 & 2.33 & 21.91 & 82.18 & 506 & 820 & $\ldots$ \\
\hline $\mathrm{N} 4395$ & 10.63 & 0.53 & $\cdots$ & $\ldots$ & $\ldots$ & 0.16 & 4.44 & 13.46 & $301^{(3)}$ & $<70$ & $\ldots$ \\
\hline N4438 & 10.48 & 0.75 & $\ldots$ & $\ldots$ & 0.15 & $\ldots$ & 4.59 & 12.54 & $4^{(3)}$ & 125 & $\ldots$ \\
\hline $\mathrm{N} 4272$ & 9.30 & 0.94 & $\ldots$ & $\ldots$ & $\ldots$ & $\ldots$ & $\ldots$ & $\ldots$ & $<4^{(3)}$ & 250 & $\ldots$ \\
\hline N4517 & 10.45 & 0.58 & $\ldots$ & $\ldots$ & 0.65 & 0.42 & 7.35 & 21.05 & $103^{(3)}$ & 130 & $\ldots$ \\
\hline N4559 & 9.96 & 0.37 & $\ldots$ & 8.34 & 0.57 & 0.62 & 10.34 & 28.15 & 314 & 180 & $\ldots$ \\
\hline N4565 & 9.64 & 0.68 & $\ldots$ & 6.70 & 1.71 & 1.39 & 9.30 & 49.68 & 242 & 100 & $\ldots$ \\
\hline N4569 & 9.91 & 0.68 & 0.24 & $\ldots$ & 0.89 & 1.10 & 9.64 & 28.51 & $12^{(3)}$ & 90 & $\ldots$ \\
\hline N4594 & 8.86 & 0.88 & $\ldots$ & $\ldots$ & 0.75 & 0.38 & 3.94 & 24.05 & $\ldots$ & 300 & $\ldots$ \\
\hline N4631 & 9.20 & 0.41 & $\ldots$ & $\ldots$ & 6.49 & 8.35 & 89.26 & 216.44 & $639^{(3)}$ & 1300 & $\ldots$ \\
\hline N4656 & 10.16 & 0.29 & $\ldots$ & $\ldots$ & 0.12 & 0.28 & 6.54 & 11.75 & $393^{(3)}$ & 118 & $\ldots$ \\
\hline $\mathrm{N} 4725$ & 9.80 & 0.71 & $\ldots$ & 7.06 & 0.32 & 0.15 & 3.97 & 21.87 & 100 & $<100$ & $\ldots$ \\
\hline N4736 & 8.78 & 0.74 & 0.15 & $\ldots$ & 5.51 & 5.76 & 68.36 & 139.52 & $62^{(3)}$ & 270 & $\ldots$ \\
\hline $\mathrm{N} 4762$ & 10.77 & 0.82 & 0.33 & $\ldots$ & $\ldots$ & $\ldots$ & $\ldots$ & $\ldots$ & $\ldots$ & $<50$ & $\ldots$ \\
\hline $\mathrm{N} 4826$ & 9.00 & 0.76 & 0.14 & 6.10 & 1.91 & 1.64 & 37.11 & 79.93 & 56 & 90 & $\ldots$ \\
\hline N4945 & 8.15 & $\ldots$ & $\ldots$ & $\ldots$ & 28.09 & 37.53 & 639.41 & 1465.39 & $\ldots$ & $6600^{(4)}$ & $\ldots$ \\
\hline N5033 & 10.38 & 0.49 & $\ldots$ & 7.50 & 1.57 & 1.47 & 18.15 & 53.24 & 178 & 200 & $\ldots$ \\
\hline N5055 & 9.13 & 0.69 & $\ldots$ & 6.30 & 6.33 & 5.81 & 39.70 & 165.61 & 372 & 409 & $\ldots$ \\
\hline N5102 & 10.06 & 0.63 & 0.22 & $\ldots$ & $\ldots$ & $\ldots$ & 0.85 & 2.86 & $89^{(3)}$ & $\ldots$ & $\ldots$ \\
\hline N5128 & 7.42 & 0.86 & $\ldots$ & $\ldots$ & 26.37 & 25.69 & 236.32 & 518.10 & $\cdots$ & $1300000^{(4)}$ & $\ldots$ \\
\hline N5170 & $11.01^{(1)}$ & $\ldots$ & $\ldots$ & 8.38 & 0.14 & $\ldots$ & 1.05 & 5.09 & 87 & $\ldots$ & $\ldots$ \\
\hline M51 & 8.83 & 0.57 & $\ldots$ & $\ldots$ & 11.25 & 12.61 & 84.95 & 192.56 & $230^{(3)}$ & 1500 & 16000 \\
\hline M83 & 8.03 & $\ldots$ & $\ldots$ & $\ldots$ & 31.24 & 41.47 & 285.97 & 661.47 & $1600^{(3)}$ & 1650 & $\ldots$ \\
\hline M101 & 8.19 & 0.46 & $\ldots$ & $\ldots$ & 7.40 & 10.29 & 92.86 & 263.48 & $3900^{(3)}$ & 850 & 11000 \\
\hline $\mathrm{U} \mathrm{Mi}$ & $11.6^{(2)}$ & $\ldots$ & $\ldots$ & $\ldots$ & $\ldots$ & $\cdots$ & $\cdots$ & $\cdots$ & $\ldots$ & $\cdots$ & $\cdots$ \\
\hline N5907 & 10.31 & 0.61 & $\ldots$ & 7.58 & 1.36 & 1.10 & 8.14 & 48.14 & 242 & 114 & $\ldots$ \\
\hline Draco Sys. & $11.9^{(2)}$ & $\ldots$ & $\ldots$ & $\ldots$ & $\ldots$ & $\ldots$ & $\ldots$ & $\ldots$ & $\ldots$ & $\ldots$ & $\ldots$ \\
\hline N6744 & 8.74 & $\ldots$ & $\ldots$ & $\ldots$ & 3.32 & 3.53 & 22.07 & 90.05 & $975^{(3)}$ & $\ldots$ & $\ldots$ \\
\hline N6822 & 8.46 & $\ldots$ & $\ldots$ & $\ldots$ & 0.29 & 2.59 & 58.59 & 196.71 & $2150^{(3)}$ & $<50$ & $\ldots$ \\
\hline N6946 & 7.97 & 0.43 & $\ldots$ & $\ldots$ & 14.36 & 18.31 & 146.54 & 357.29 & $839^{(3)}$ & 1500 & 21000 \\
\hline I5201 & 11.06 & $\ldots$ & $\ldots$ & $\ldots$ & 0.12 & $\ldots$ & 1.56 & 3.42 & $\ldots$ & $\ldots$ & $\ldots$ \\
\hline N7331 & 9.67 & 0.69 & 0.12 & 6.44 & 3.82 & 3.48 & 36.59 & 120.33 & 212 & 486 & $\ldots$ \\
\hline N7640 & 10.41 & 0.32 & $\ldots$ & 8.97 & 0.14 & $\ldots$ & 3.91 & 11.95 & $360^{(3)}$ & $\ldots$ & $\ldots$ \\
\hline N7793 & 9.59 & 0.56 & -0.12 & 7.89 & 1.77 & 1.75 & 20.80 & 58.70 & 271 & $\ldots$ & $\ldots$ \\
\hline WLM & 10.94 & 0.29 & -0.32 & $\ldots$ & $\ldots$ & $\ldots$ & 0.32 & 1.09 & $285^{(3)}$ & $\ldots$ & $\ldots$ \\
\hline
\end{tabular}

Notes.-Optical values have been corrected using the Galactic reddening estimates of Burstein and Heiles 1984. Units: $1.65 \mu \mathrm{m}$ (mag); $\mathrm{H} \mathrm{I}\left(\mathrm{Jy} \mathrm{km} \mathrm{s}^{-1}\right) ; 21 \mathrm{~cm}^{(\mathrm{mJy})}$; $\mathrm{CO}\left(\mathrm{mJy} \mathrm{km} \mathrm{s}^{-1}\right)$.

SourCES.-Optical: RC2; (1) RSA; (2) Kraan-Korteweg and Tammann 1979. $1.65 \mu \mathrm{m}$ : Aaronson et al. 1982. H I: Aaronson et al. 1982; (3) Huchtmeier et al. 1983. $21 \mathrm{~cm}$ continuum: Hummel 1980; (4) RC2. CO: Sanders and Young 1986; (5) Verter 1985.

entries. The warm component dust temperature statistics exclude the values determined using a limit.

\section{d) Far-Infrared Diameters}

The far-infrared isophotal diameter of the largest infrared extended galaxies least affected by the IRAS beam was obtained from the $60 \mu \mathrm{m}$ surface brightness map. The diameter was measured along the galaxy optical major axis to the 25 mJy $\operatorname{arcmin}^{-2}$ isophotal brightness level. Table 5 reports the results. The column entries are:

Column (1).-Gàlaxy name.

Column (2).- The RC2 morphological classification $T$ index and bar parameter letter.

Column (3). $-D_{\mathrm{IR}}$, the $60 \mu \mathrm{m}$ isophotal diameter.

Column (4). $-D_{\mathrm{IR}} / D_{B}$, the ratio of the $60 \mu \mathrm{m}$ isophotal diameter to the blue-light isophotal major diameter statistically corrected for Galactic extinction. The corrected blue diameter was calculated using the interpolation formula 
adopted from RC2:

$$
\log D_{B}=\log D_{25}+A_{B}(0.12-0.007 T),
$$

where $A_{B}$ is the Galactic reddening from Burstein and Heiles (1984) and $T$ is the RC2 morphological classification $T$ index.

Column (5).- $-\left(A_{e} / D\right)_{\mathrm{IR}}$, the ratio of the "effective" aperture diameter to the isophotal diameter of the $60 \mu \mathrm{m}$ emission. As in $\mathrm{RC} 2$, the $60 \mu \mathrm{m}$ emission effective aperture is defined here as the circular aperture, centered on the galaxy image, from which one-half of the galaxy total $60 \mu \mathrm{m}$ emission was measured.

Column (6).- $\left(A_{e} / D\right)_{B}$, the ratio of the effective aperture diameter of the isophotal major diameter of the blue-light emission. Both diameters were obtained from RC2.

Uncertain infrared diameter measurements, possibly affected by the beam or by confusion due to local "cirrus" emission, are followed by a colon. The last two lines report the mean and standard deviation of the column entries.

TABLE 4

INTEGRATED INFRARED PROPERTIES

\begin{tabular}{|c|c|c|c|c|c|c|c|c|c|c|}
\hline $\begin{array}{c}\text { Name } \\
(1)\end{array}$ & 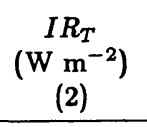 & $\begin{array}{c}L\left(I R_{T}\right) \\
\left(L_{\odot}\right) \\
(3)\end{array}$ & $\begin{array}{r}f_{C, T} \\
(4) \\
\end{array}$ & $\begin{array}{c}F_{\mathrm{IR}} \\
\left(\mathrm{W} \mathrm{m}^{-2}\right) \\
(5)\end{array}$ & $\begin{array}{c}L\left(F_{\mathrm{IR}}\right) \\
\left(L_{\odot}\right) \\
(6) \\
\end{array}$ & $\begin{array}{r}f_{\mathrm{FIR}, C} \\
(7) \\
\end{array}$ & $\begin{array}{r}f_{T, B} \\
(8) \\
\end{array}$ & $\begin{array}{r}f_{\mathrm{FIR}, B} \\
(9) \\
\end{array}$ & $\begin{array}{l}T_{W} \\
(\mathrm{~K}) \\
(10) \\
\end{array}$ & $\begin{array}{l}T_{C} \\
(\mathrm{~K}) \\
(11)\end{array}$ \\
\hline $\mathrm{N} 45$ & $2.04 \mathrm{E}-13$ & $7.25 \mathrm{E}+08$ & 0.897 & $1.16 \mathrm{E}-13$ & $4.10 \mathrm{E}+08$ & 0.631 & 0.201 & 0.114 & 16.1: & 00.7 \\
\hline & & & 0.875 & & $1.47 \mathrm{E}+09$ & & 0.257 & & & 34.1 \\
\hline N134 & $2.87 \mathrm{E}-12$ & $9.13 \mathrm{E}+10$ & 0.779 & $1.33 \mathrm{E}-12$ & $4.23 \mathrm{E}+10$ & 0.595 & 2.064 & 0.956 & 210.3 & 28.5 \\
\hline N185 & $6 E-14$ & $1.59 \mathrm{E}+06$ & 0.818 & $3.44 \mathrm{E}-14$ & $5.73 \mathrm{E}+05$ & 0.440 & 0.021 & 0.007 & 406.2: & 23.7 \\
\hline N205 & $8 E-13$ & & 0.755 & $6.71 \mathrm{E}-14$ & $1.12 \mathrm{E}+06$ & 0.428 & 0.026 & 0.009 & 224.0 & 23.2 \\
\hline M31 & & & 0.685 & $5.43 \mathrm{E}-11$ & $9.05 \mathrm{E}+08$ & 0.473 & 0.207 & 0.067 & 295.6 & 24.6 \\
\hline N247 & & & 0.980 & $6.02 \mathrm{E}-13$ & $1.88 \mathrm{E}+08$ & 0.602 & 0.168 & 0.099 & 224.4: & 29.2 \\
\hline $\mathrm{N} 253$ & & & 0.735 & $5.59 \mathrm{E}-11$ & $3.04 \mathrm{E}+10$ & 0.741 & 4.742 & 2.583 & 172.8 & 36.8 \\
\hline & & & 0.934 & & & & 0.235 & 0.153 & 156.5 & 34.4 \\
\hline N 300 & $2.90 \mathrm{E}-12$ & & & & $3.04 \mathrm{E}+08$ & 0.619 & 0.304 & 0.177 & 230.4 & 29.9 \\
\hline 13 & 13 & $41 E+06$ & 0.913 & $27 E-14$ & +06 & 0.671 & 0.060 & 0.037 & 183.0: & 32.4 \\
\hline & & & 08 & & 08 & & 0.556 & 87 & & \\
\hline & 12 & 10 & 0.785 & 12 & +10 & & 0.885 & 5 & & .1 \\
\hline $\mathrm{N} \epsilon$ & -12 & -10 & 0.802 & -12 & +10 & & 6.569 & 9 & & 0.1 \\
\hline & -12 & +10 & 0.787 & -12 & -10 & & 4.031 & 962 & & 9.6 \\
\hline & & 09 & 0.890 & -13 & -09 & 0.600 & 0.535 & 0.285 & 17 & 3.8 \\
\hline & & 11 & 0.758 & -12 & +10 & 0.687 & 2.502 & 1.304 & 174.1 & 32.5 \\
\hline 1 & & +09 & 0.875 & -13 & +09 & 0.460 & 0.107 & 0.043 & 251.8 & 24.3 \\
\hline $\mathrm{N}$ & 12 & 09 & 0.858 & -12 & $E+09$ & 0.678 & 0.829 & 0.483 & 158.5 & 32.4 \\
\hline N1365 & 9. & & 0.759 & -12 & +11 & 0.714 & 3.665 & 1.985 & 169.1 & 34.2 \\
\hline I342 & תח & 10 & 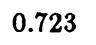 & -11 & $4 \mathrm{E}+10$ & 0. & 1.785 & 0.875 & 194.0 & 32.1 \\
\hline & & & & & & & 1.309 & 88 & & 29.0 \\
\hline & 13 & 08 & 0.962 & -13 & & & 0.234 & 3 & & 33.2 \\
\hline $\mathrm{LI}$ & 9 & & 0.839 & -09 & & & 0.471 & & & .4 \\
\hline & 12 & & 0.8 & -12 & & & 0.724 & & & 0.9 \\
\hline 3 & 12 & & 0 & 13 & -09 & & 0.662 & & & 7.4 \\
\hline $\mathrm{N} 2$ & -12 & 7.8 & 68 & 13 & 09 & 1 & 0.442 & & & .5 \\
\hline N2 & -12 & 10 & 0.751 & -12 & 09 & 5 & 1.444 & 0.711 & 20 & 1.2 \\
\hline N2997 & -12 & +10 & 0.733 & -12 & +10 & 71 & 1.800 & 86 & 20 & 32.0 \\
\hline M81 & $7 E-12$ & $3.29 \mathrm{~F}$ & 0.774 & -12 & $1.45 \mathrm{E}+09$ & 0.569 & 0.312 & 0.138 & 254.2 & 27.7 \\
\hline & & & 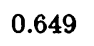 & & & & 17.602 & 8.760 & 3 & 47.6 \\
\hline N3109 & -13 & +07 & 0.968 & -13 & $4.39 \mathrm{E}+07$ & 0.691 & 0.099 & 0.066 & 152.3: & 34.0 \\
\hline N31 & -13 & +09 & 0.834 & -13 & $2.67 \mathrm{E}+09$ & 0.661 & 0.602 & 0.332 & 198.5 & 31.7 \\
\hline I 2574 & & & & -13 & $8.12 \mathrm{E}+07$ & 0.535 & 0.320 & 0.166 & 164.6: & 26.6 \\
\hline N35 & 2 & 3. & & -12 & $1.48 \mathrm{E}+10$ & 0.651 & 1.495 & 0.728 & 259.5 & 31.3 \\
\hline N35 & & 3.0 & & & $1.61 \mathrm{E}+10$ & 0.682 & 2.011 & 1.065 & 197.6 & 32.7 \\
\hline N362 & -12 & & & & +09 & & 1.171 & & & 30.2 \\
\hline N3623 & $09 E-13$ & & & & & & 0.200 & & & 25.3 \\
\hline N3627 & $7.03 E-12$ & & & & +10 & & 1.689 & & & 32.3 \\
\hline N3628 & $5.74 \mathrm{E}-12$ & $3.71 \mathrm{E}+10$ & 0.798 & -12 & $2.01 \mathrm{E}+10$ & 0.680 & 1.721 & 0.933 & 5 & 2.7 \\
\hline N3718 & & & & & & & & & & 0.7 \\
\hline $\mathrm{N} 4192$ & $1.07 \mathrm{E}-12$ & $1.60 \mathrm{E}+10$ & 0.793 & $5.26 \mathrm{E}-13$ & $7.87 \mathrm{E}+09$ & 0.619 & 0.612 & 0.300 & 325.1 & 29.9 \\
\hline $\mathrm{N} 4216$ & $5.26 \mathrm{E}-13$ & $7.87 \mathrm{E}+09$ & 0.957 & $2.35 \mathrm{E}-13$ & $3.51 \mathrm{E}+09$ & 0.467 & 0.326 & 0.146 & 202.9: & 24.5 \\
\hline $\mathrm{N} 4236$ & $4.59 \mathrm{E}-13$ & $1.41 \mathrm{E}+08$ & 0.810 & $2.56 \mathrm{E}-13$ & $7.88 \mathrm{E}+07$ & 0.688 & 0.126 & 0.070 & 143.7 & 32.2 \\
\hline N4244 & $5.99 \mathrm{E}-13$ & $4.64 \mathrm{E}+08$ & 0.986 & $3.39 E-13$ & $2.63 \mathrm{E}+08$ & 0.574 & 0.218 & 0.123 & 219.5: & 28.1 \\
\hline
\end{tabular}


TABLE 4-Continued

\begin{tabular}{|c|c|c|c|c|c|c|c|c|c|c|}
\hline $\begin{array}{c}\text { Name } \\
(1)\end{array}$ & $\begin{array}{c}I R_{T} \\
\left(\mathrm{~W} \mathrm{~m}^{-2}\right) \\
(2)\end{array}$ & $\begin{array}{c}L\left(I R_{T}\right) \\
\left(L_{\odot}\right) \\
(3) \\
\end{array}$ & $\begin{array}{r}f_{C, T} \\
(4) \\
\end{array}$ & $\begin{array}{c}F_{\mathrm{IR}} \\
\left(\mathrm{W} \mathrm{\textrm {m } ^ { - 2 }}\right) \\
(5)\end{array}$ & $\begin{array}{c}L\left(F_{\mathrm{IR}}\right) \\
\left(L_{\odot}\right) \\
(6) \\
\end{array}$ & $\begin{array}{r}f_{\mathrm{FIR}, C} \\
(7) \\
\end{array}$ & $\begin{array}{r}f_{T, B} \\
(8)\end{array}$ & $\begin{array}{r}f_{\mathrm{FIR}, B} \\
(9)\end{array}$ & $\begin{array}{l}T_{W} \\
(\mathrm{~K}) \\
(10)\end{array}$ & $\begin{array}{l}T_{C} \\
(\mathrm{~K}) \\
(11) \\
\end{array}$ \\
\hline $\mathrm{N} 4258$ & $3.63 \mathrm{E}-12$ & $1.23 \mathrm{E}+10$ & 0.791 & $1.69 \mathrm{E}-12$ & $5.71 \mathrm{E}+09$ & 0.589 & 0.441 & 0.205 & 225.5 & 28.4 \\
\hline $\mathrm{N} 4395$ & $5.02 \mathrm{E}-13$ & $5.80 \mathrm{E}+08$ & 0.943 & $2.99 \mathrm{E}-13$ & $3.46 \mathrm{E}+08$ & 0.632 & 0.374 & 0.223 & 167.1: & 30.4 \\
\hline N4438 & $5.15 \mathrm{E}-13$ & $7.71 \mathrm{E}+09$ & 0.868 & $2.91 \mathrm{E}-13$ & $4.35 \mathrm{E}+09$ & 0.651 & 0.334 & 0.189 & 382.0: & 31.6 \\
\hline N4517 & $9.38 \mathrm{E}-13$ & $7.44 \mathrm{E}+09$ & 0.794 & $4.79 \mathrm{E}-13$ & $3.80 \mathrm{E}+09$ & 0.644 & 0.592 & 0.303 & 262.2 & 31.0 \\
\hline N4559 & $1.18 \mathrm{E}-12$ & $8.73 E+09$ & 0.852 & $6.56 \mathrm{E}-13$ & $4.87 \mathrm{E}+09$ & 0.655 & 0.473 & 0.264 & 212.1 & 31.5 \\
\hline N4565 & $2.30 \mathrm{E}-12$ & $3.94 \mathrm{E}+10$ & 0.782 & $9.15 \mathrm{E}-13$ & $1.57 \mathrm{E}+10$ & 0.509 & 0.688 & 0.274 & 235.7 & 25.6 \\
\hline N4569 & $1.28 \mathrm{E}-12$ & $1.92 \mathrm{E}+10$ & 0.780 & $6.43 E-13$ & $9.62 \mathrm{E}+09$ & 0.642 & 0.494 & 0.247 & 201.5 & 30.5 \\
\hline N4594 & $1.13 \mathrm{E}-12$ & $1.07 \mathrm{E}+10$ & 0.789 & $4.27 \mathrm{E}-13$ & $4.06 \mathrm{E}+09$ & 0.479 & 0.165 & 0.062 & 292.3 & 24.8 \\
\hline $\mathrm{N} 4631$ & $9.91 \mathrm{E}-12$ & $4.55 \mathrm{E}+10$ & 0.789 & $5.32 \mathrm{E}-12$ & $2.44 \mathrm{E}+10$ & 0.681 & 1.980 & 1.063 & 199.9 & 32.7 \\
\hline N4656 & $5.14 \mathrm{E}-13$ & $2.50 \mathrm{E}+09$ & 0.904 & $3.36 \mathrm{E}-13$ & $1.64 \mathrm{E}+09$ & 0.723 & 0.249 & 0.163 & 171.9 & 36.6 \\
\hline $\mathrm{N} 4725$ & $8.98 \mathrm{E}-13$ & $1.53 \mathrm{E}+10$ & 0.883 & $3.98 \mathrm{E}-13$ & $6.77 \mathrm{E}+09$ & 0.502 & 0.312 & 0.138 & 303.9 & 25.6 \\
\hline N4736 & $6.93 \mathrm{E}-12$ & $1.03 \mathrm{E}+10$ & 0.760 & $3.73 \mathrm{E}-12$ & $5.56 \mathrm{E}+09$ & 0.709 & 0.941 & 0.507 & 216.0 & 34.8 \\
\hline $\mathrm{N} 4826$ & $3.54 \mathrm{E}-12$ & $5.43 \mathrm{E}+09$ & 0.841 & $2.08 \mathrm{E}-12$ & $3.18 \mathrm{E}+09$ & 0.696 & 0.589 & 0.345 & 234.7 & 34.2 \\
\hline N4945 & $6.28 \mathrm{E}-11$ & $9.40 \mathrm{E}+10$ & 0.855 & $3.70 \mathrm{E}-11$ & $5.53 \mathrm{E}+10$ & 0.688 & 4.772 & 2.806 & 198.1 & 33.4 \\
\hline N5033 & $2.34 \mathrm{E}-12$ & $2.36 \mathrm{E}+10$ & 0.800 & $1.20 \mathrm{E}-12$ & $1.21 \mathrm{E}+10$ & 0.641 & 1.388 & 0.712 & 223.7 & 30.7 \\
\hline N5055 & $7.69 \mathrm{E}-12$ & $2.91 \mathrm{E}+10$ & 0.756 & $3.29 \mathrm{E}-12$ & $1.24 \mathrm{E}+10$ & 0.566 & 1.441 & 0.616 & 224.7 & 27.5 \\
\hline N5102 & $1.17 \mathrm{E}-13$ & $1.76 \mathrm{E}+08$ & 0.851 & $6.11 \mathrm{E}-14$ & $9.14 \mathrm{E}+07$ & 0.611 & 0.052 & 0.027 & 216.4: & 29.4 \\
\hline N5128 & $2.70 \mathrm{E}-11$ & $4.05 \mathrm{E}+10$ & 0.708 & $1.34 \mathrm{E}-11$ & $2.00 \mathrm{E}+10$ & 0.699 & 1.048 & 0.519 & 220.9 & 33.9 \\
\hline N5170 & $2.33 E-13$ & $4.81 \mathrm{E}+09$ & 0.778 & $9.61 \mathrm{E}-14$ & $1.98 \mathrm{E}+09$ & 0.531 & 0.246 & 0.102 & 329.7: & 26.5 \\
\hline M51 & $1.05 \mathrm{E}-11$ & $3.85 \mathrm{E}+10$ & 0.669 & $4.91 \mathrm{E}-12$ & $1.80 \mathrm{E}+10$ & 0.697 & 1.493 & 0.697 & 209.4 & 33.4 \\
\hline M83 & $3.42 \mathrm{E}-11$ & $1.05 \mathrm{E}+11$ & 0.703 & $1.67 \mathrm{E}-11$ & $5.13 \mathrm{E}+10$ & 0.694 & 2.327 & 1.135 & 197.5 & 33.1 \\
\hline M101 & $1.18 \mathrm{E}-11$ & $2.12 \mathrm{E}+10$ & 0.791 & $6.05 \mathrm{E}-12$ & $1.09 \mathrm{E}+10$ & 0.651 & 0.926 & 0.476 & 194.3 & 30.9 \\
\hline N5907 & $2.17 \mathrm{E}-12$ & $1.65 \mathrm{E}+10$ & 0.816 & $8.62 \mathrm{E}-13$ & $6.54 \mathrm{E}+09$ & 0.486 & 1.206 & 0.479 & 236.1 & 25.0 \\
\hline N6744 & $4.17 \mathrm{E}-12$ & $2.29 \mathrm{E}+10$ & 0.757 & $1.80 \mathrm{E}-12$ & $9.91 \mathrm{E}+09$ & 0.571 & 0.545 & 0.236 & 212.6 & 27.6 \\
\hline N6822 & $7.23 \mathrm{E}-12$ & $1.04 \mathrm{E}+08$ & 0.950 & $4.21 \mathrm{E}-12$ & $6.08 \mathrm{E}+07$ & 0.613 & 0.730 & 0.425 & 122.1 & 29.3 \\
\hline N6946 & $1.75 \mathrm{E}-11$ & $2.47 \mathrm{E}+10$ & 0.736 & $8.78 \mathrm{E}-12$ & $1.24 \mathrm{E}+10$ & 0.682 & 1.125 & 0.565 & 200.2 & 32.5 \\
\hline I5201 & $2.08 \mathrm{E}-13$ & $1.38 \mathrm{E}+09$ & 0.612 & $8.79 \mathrm{E}-14$ & $5.82 \mathrm{E}+08$ & 0.691 & 0.230 & 0.097 & 463.0: & 34.0 \\
\hline N7331 & $5.34 \mathrm{E}-12$ & $8.28 \mathrm{E}+10$ & 0.788 & $2.60 \mathrm{E}-12$ & $4.03 E+10$ & 0.617 & 1.644 & 0.800 & 225.6 & 29.6 \\
\hline N7640 & $4.73 \mathrm{E}-13$ & $2.73 \mathrm{E}+09$ & 0.889 & $2.65 \mathrm{E}-13$ & $1.48 \mathrm{E}+09$ & 0.629 & 0.288 & 0.161 & 308.1: & 30.4 \\
\hline N7793 & $2.61 \mathrm{E}-12$ & $3.57 \mathrm{E}+09$ & 0.796 & $1.35 \mathrm{E}-12$ & $1.85 \mathrm{E}+09$ & 0.649 & 0.746 & 0.386 & 219.1 & 31.1 \\
\hline WLM & $6.03 \mathrm{E}-14$ & $4.82 \mathrm{E}+06$ & 0.628 & $2.35 \mathrm{E}-14$ & $1.88 \mathrm{E}+06$ & 0.621 & 0.060 & 0.023 & 202.9: & 29.1 \\
\hline Median & & $8.60 \mathrm{E}+09$ & 0.796 & & $4.35 \mathrm{E}+09$ & 0.642 & 0.592 & 0.295 & 212.1 & 30.7 \\
\hline Mean & & & 0.811 & & & 0.625 & & & 215.0 & 30.6 \\
\hline RMS & & & 0.086 & & & 0.079 & & & 42.7 & 3.9 \\
\hline
\end{tabular}

NOTES. $-f_{C, T} \equiv I R_{C} / I R_{T}$, the ratio of the total flux of the cold component distribution to the total infrared flux. $f_{\mathrm{FIR}, C} \equiv F_{\mathrm{IR}} / I R_{C}$, the ratio of the 42.5 to $122.5 \mu \mathrm{m}$ flux to the total flux of the cold component distribution. $f_{T, B} \equiv I R_{T} / F_{B}{ }^{*}$, the ratio of the total infrared flux to the blue light flux (see $\S \mathrm{IV} . \mathrm{C}$ ). $f_{\mathrm{FIR}, B} \equiv F_{\mathrm{IR}} / F_{B}{ }^{*}$.

\section{ATLAS OF INFRARED PROFILES AND MAPS}

\section{a) Profile Plots}

Radial infrared brightness profiles along the IRAS scanning direction are displayed in Figure 1 for all detected galaxies, excluding the SMC and LMC. (The profile plots of surface brightness images with peak intensities less than $6 \sigma$ are not displayed.) The profiles are centered on the optical center of the galaxies and are scaled by the peak intensity found along the cut. The position angle of the in-scan cuts is noted above each set of plots. The wavelength profile of IRC +10011 , a representative IRAS point source, is also displayed.

\section{b) IRAS Surface Brightness Maps}

The atlas plates (Figs. 2-33 [P1s. 136-168]) display optical light photographs and IRAS surface brightness contour maps of the catalog galaxies for which interesting spatial structure was observed. Table 6 lists the galaxies displayed. Except for M31, the orientation of the optical photographs and infrared maps is (equatorial) north to the top and east to the left. The orientation of the M31 images is marked on the optical photograph.

Color-coded IRAS images are displayed in Figure 34 (Plate 169) for M31 (Fig. 34a), the SMC (Fig. 34b), and the LMC (Fig. $34 c$ ). These images were produced by combining the 12 (coded blue), 60 (coded green), and $100 \mu \mathrm{m}$ (coded red) maps after smoothing the 12 and $60 \mu \mathrm{m}$ maps to the resolution of the $100 \mu \mathrm{m}$ map.

\section{i) Optical Photographs}

The optical photographs are reproductions of Palomar Sky Survey or ESO blue prints. The mosaics of the SMC and 


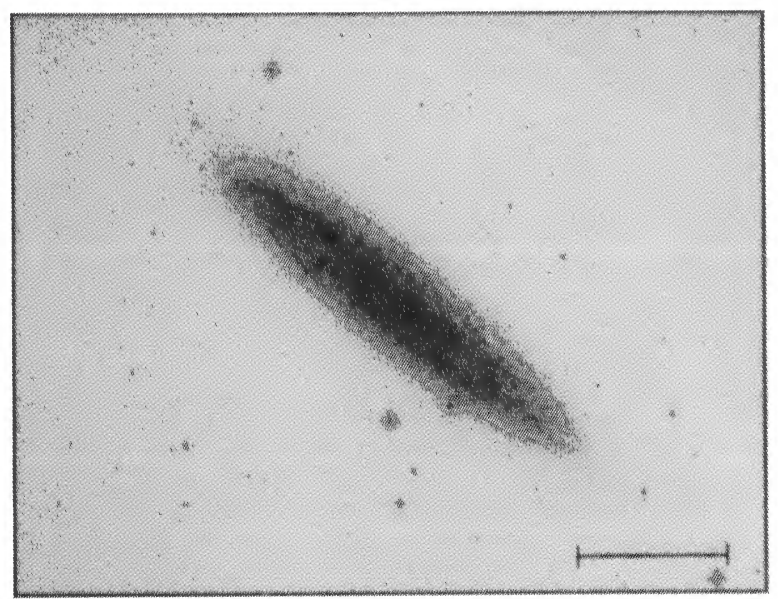

Blue Light

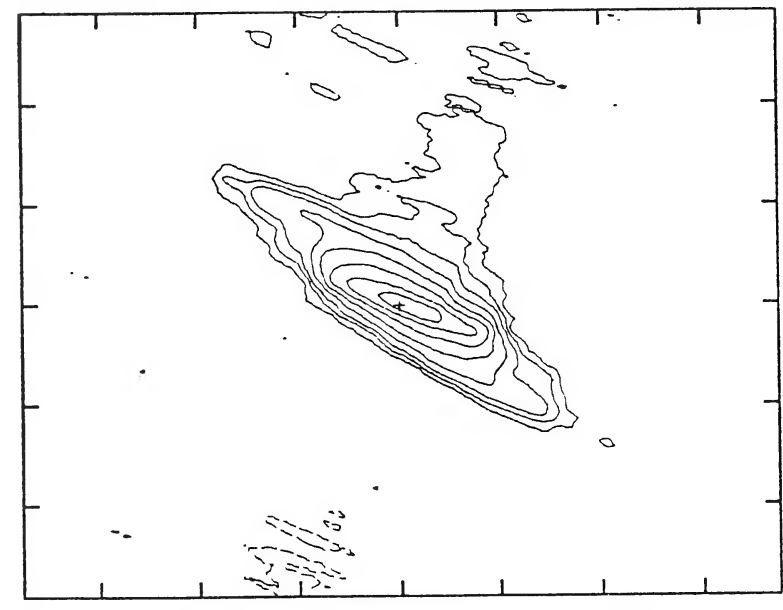

$12 \mu \mathrm{m}$

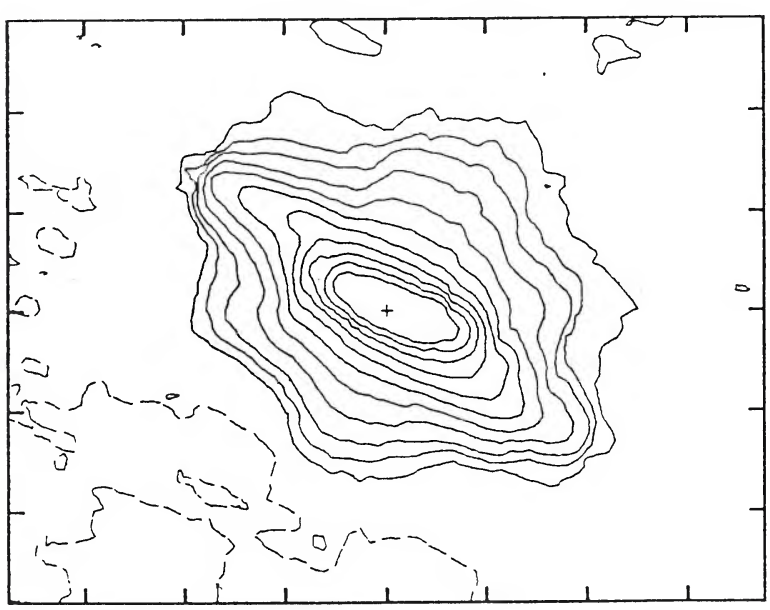

$60 \mu \mathrm{m}$

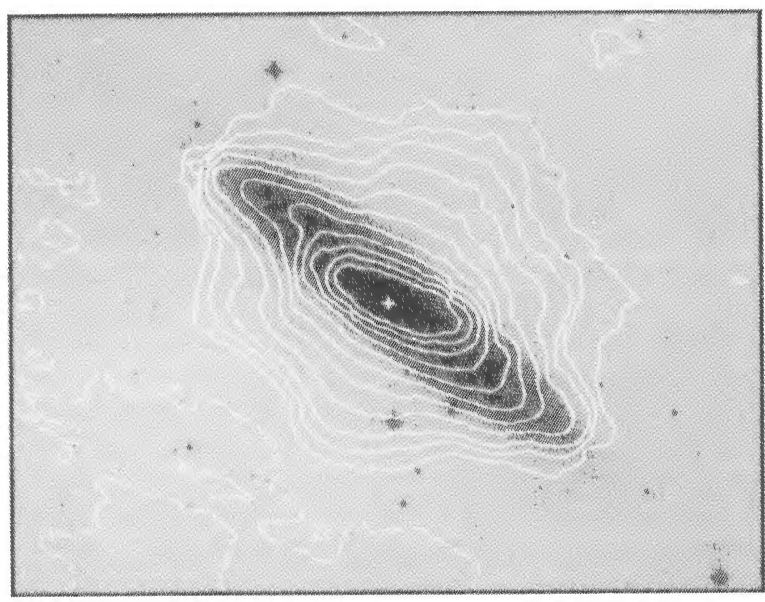

Blue and $60 \mu \mathrm{m}$
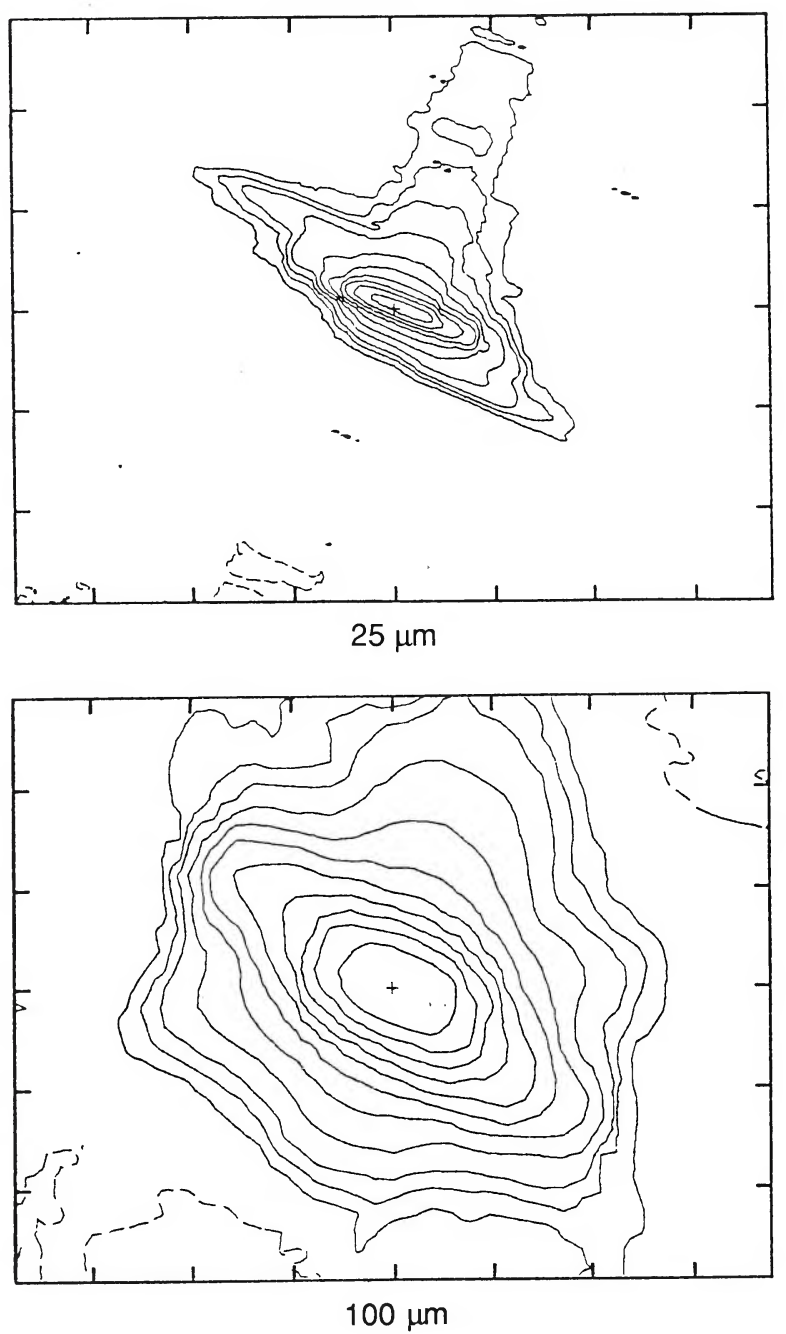

FIG. 5.-NGC 253. The tick marks on the infrared maps are spaced by 5:4. (Optical photographs reproduced by permission of the European Southern Observatory.)

Rice et al. (see 68, 102) 

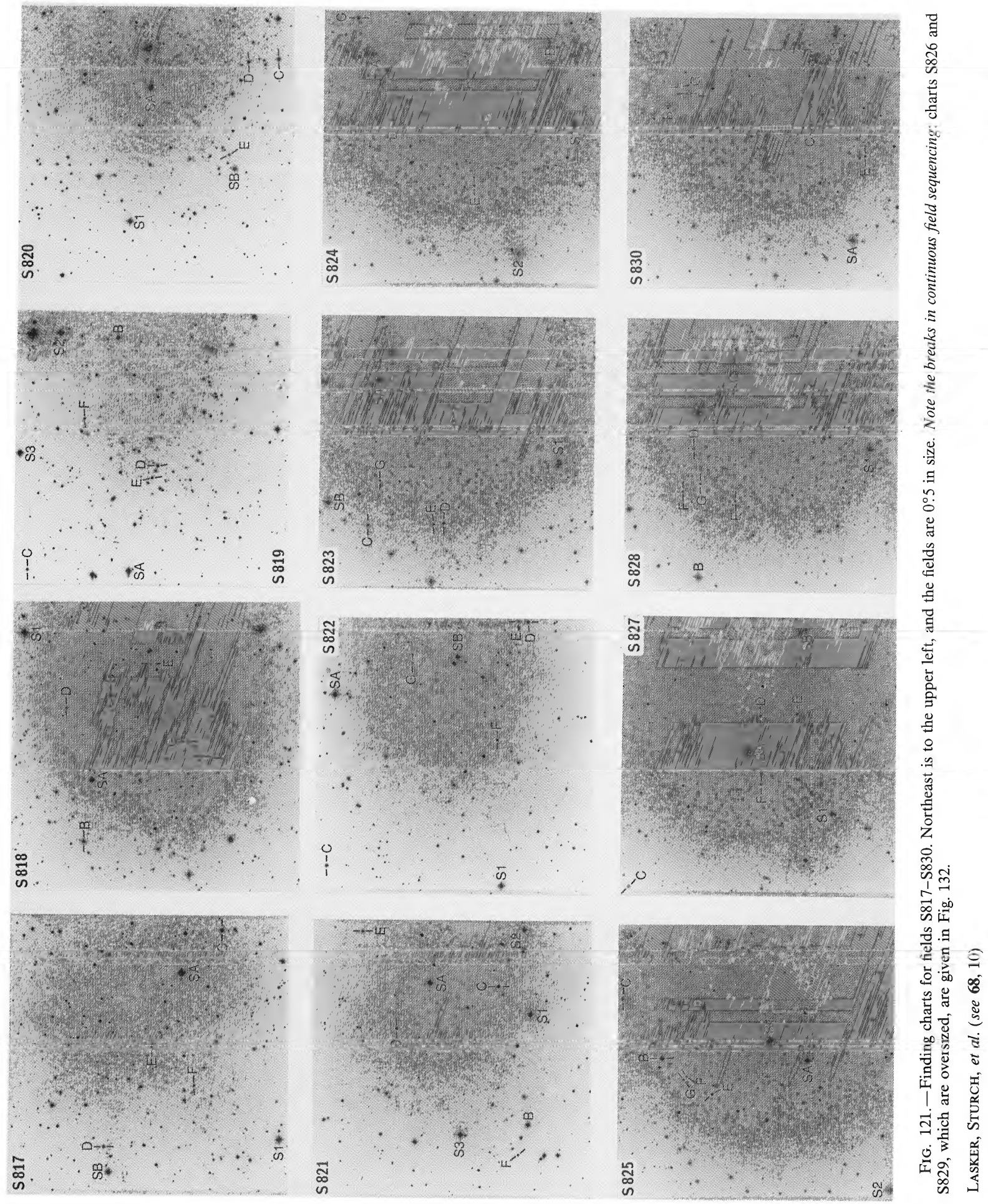


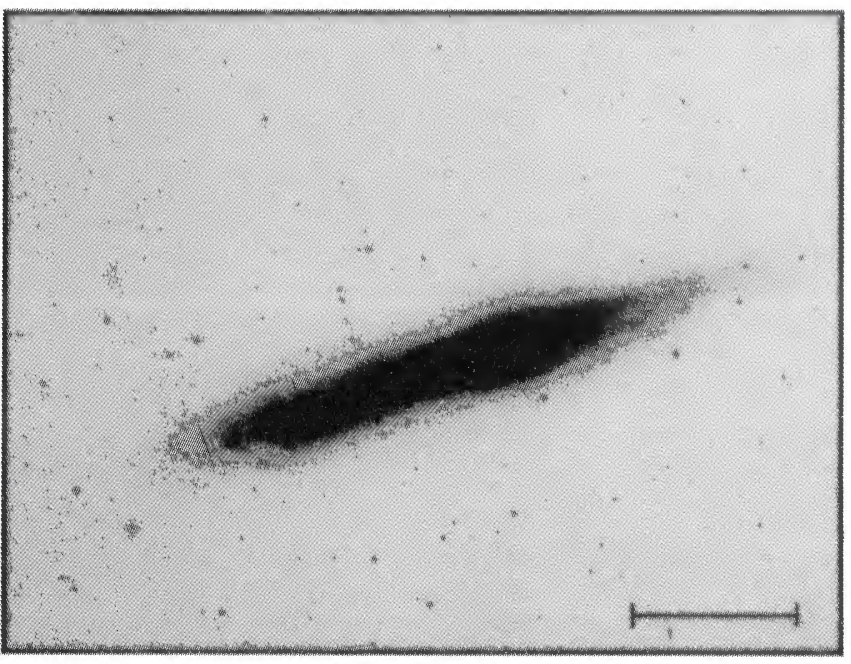

Blue Light

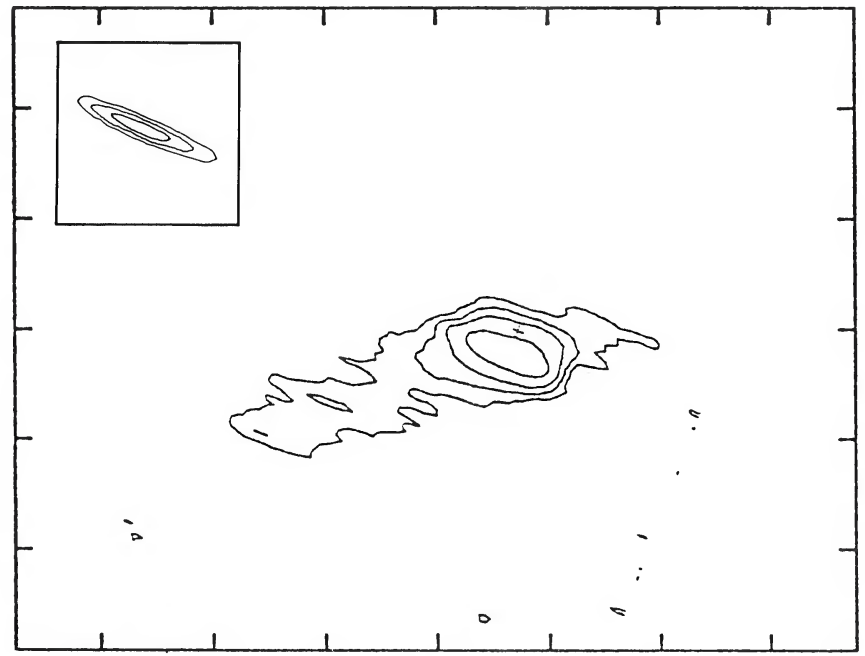

$12 \mu \mathrm{m}$

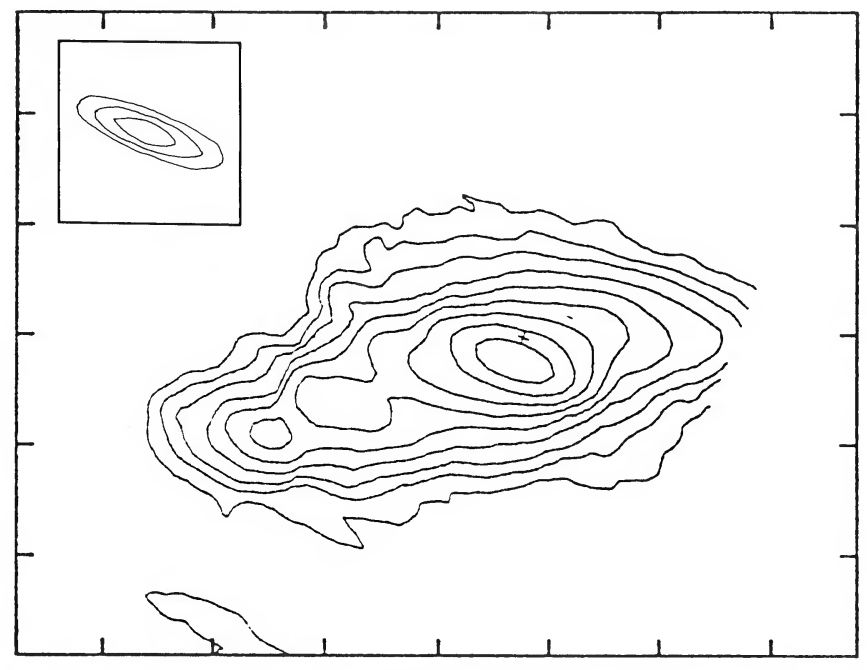

$60 \mu \mathrm{m}$

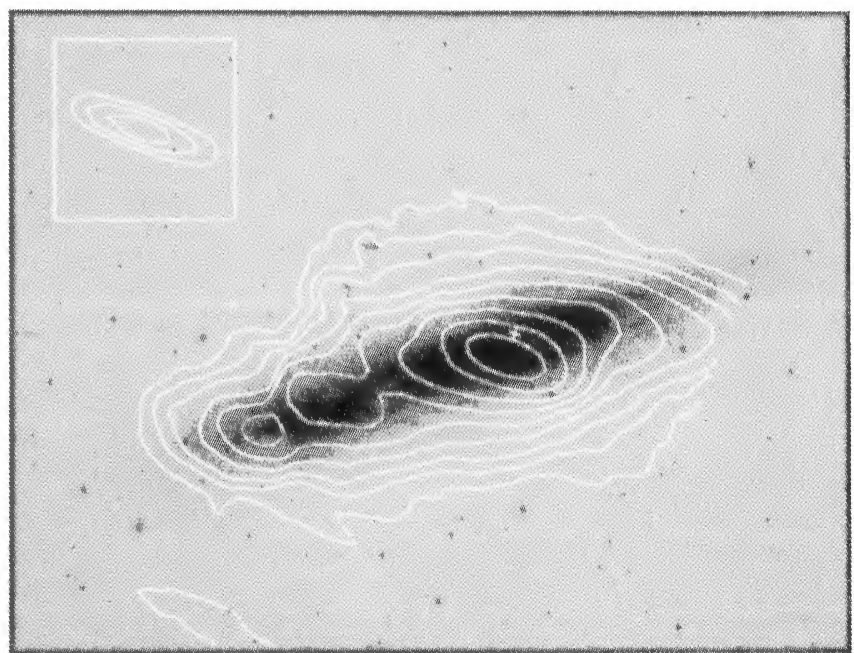

Blue and $60 \mu \mathrm{m}$

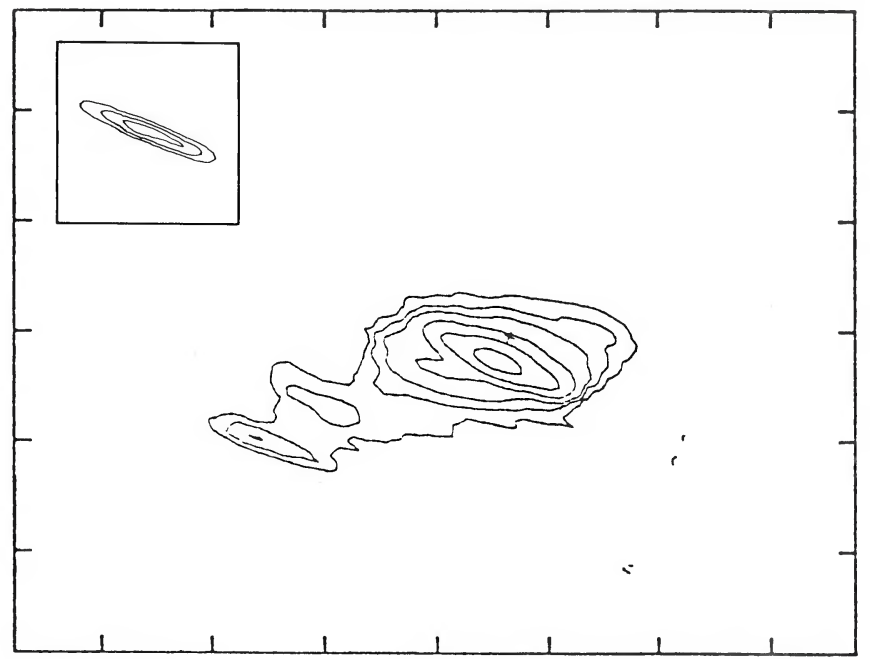

$25 \mu \mathrm{m}$

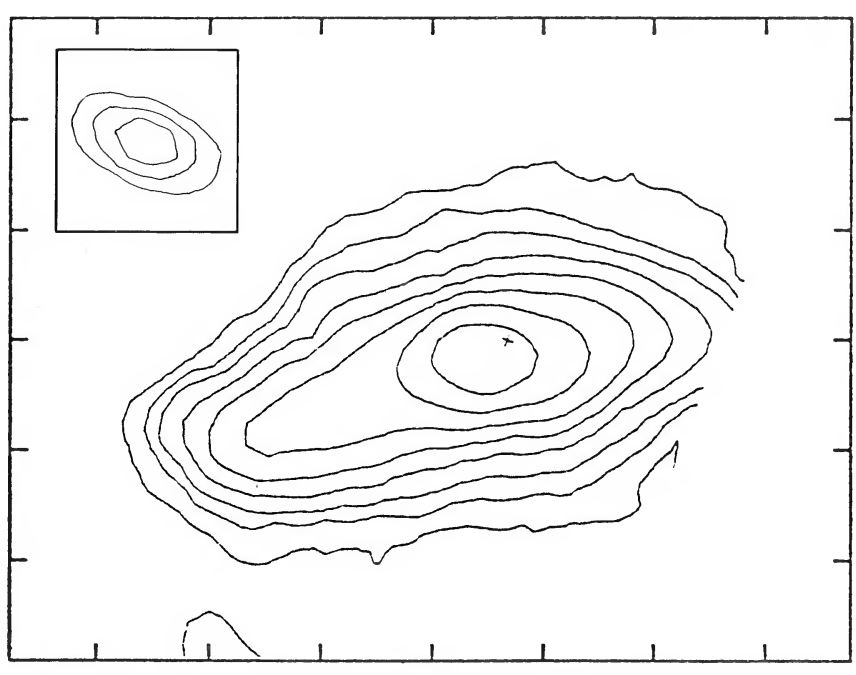

$100 \mu \mathrm{m}$

FIG. 2.-NGC 55. The tick marks on the infrared maps are spaced by 5'4. The boxed image in the upper left-hand corner of the contour maps is the IRAS beam represented by the $\frac{1}{4}, \frac{1}{2}$, and $\frac{3}{4}$ power brightness level contours of the star IRC +10011 , a representative IRAS point source. (Optical photographs reproduced by permission of the European Southern Observatory.)

RicE et al. (see 68, 102) 


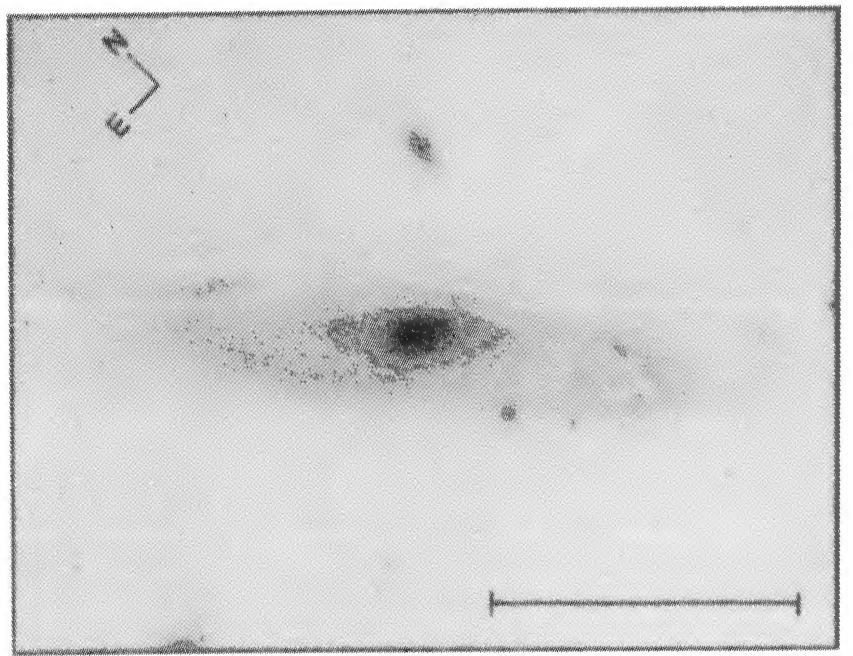

Blue Light

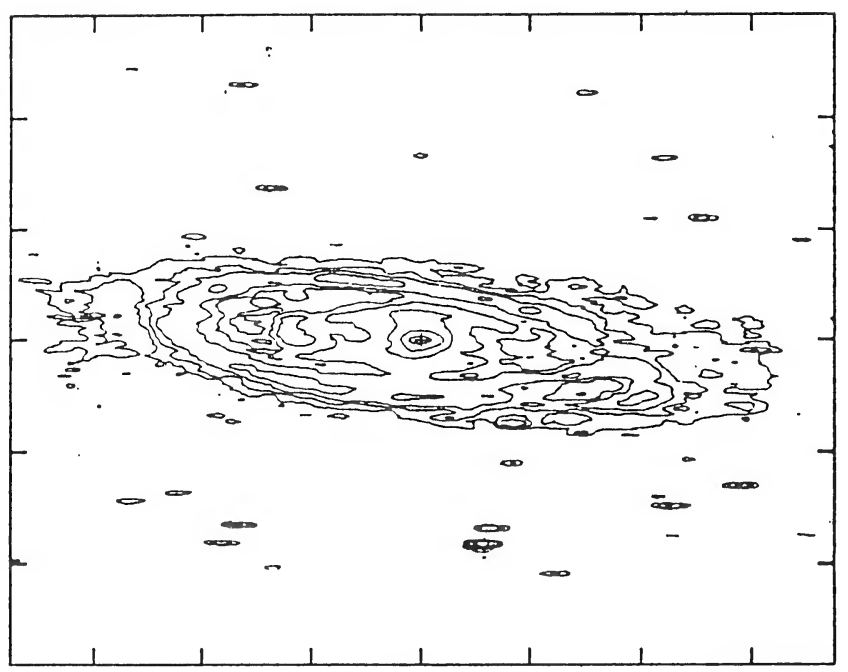

$12 \mu \mathrm{m}$

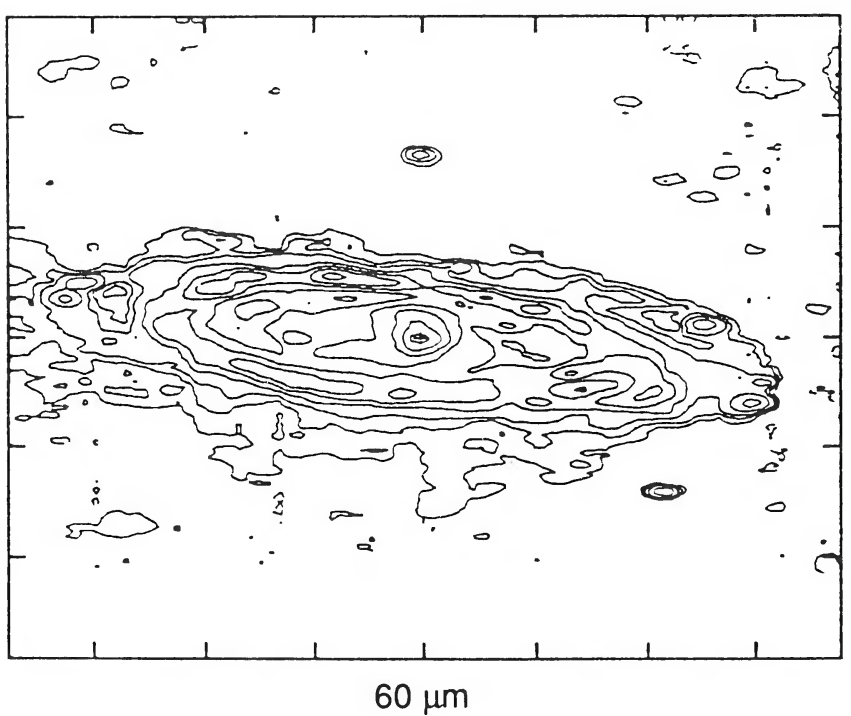

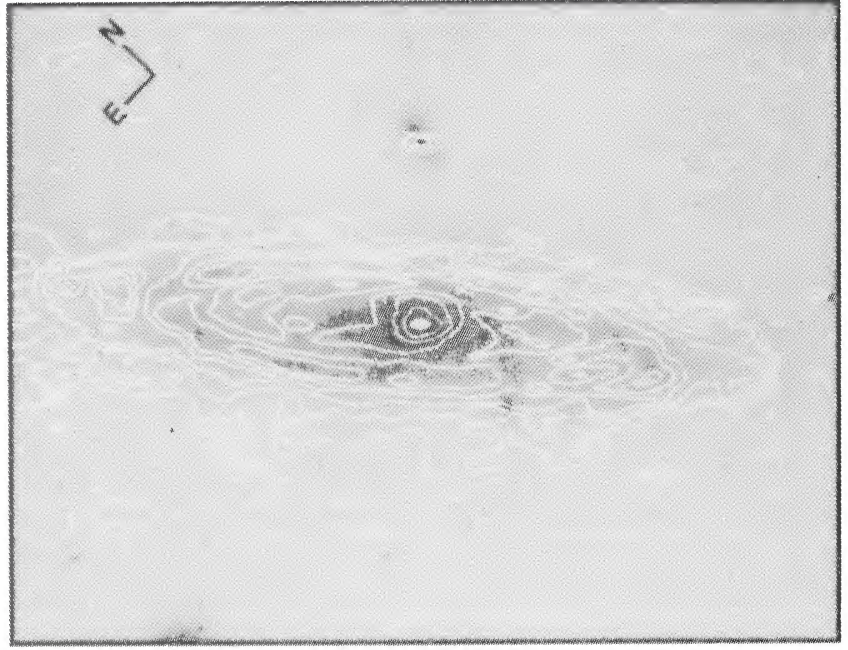

Blue and $60 \mu \mathrm{m}$

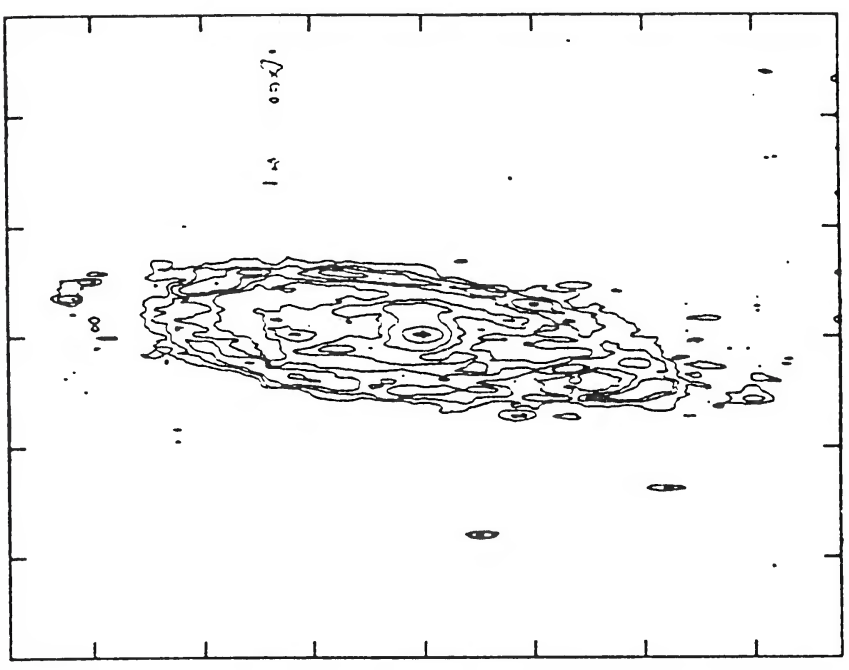

$25 \mu \mathrm{m}$

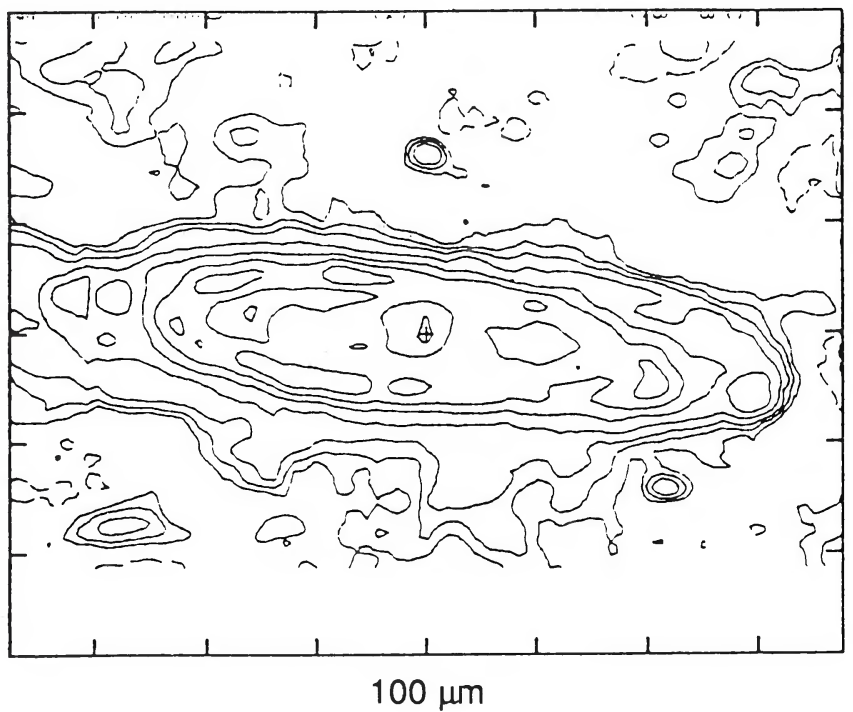

FIG. 3.-NGC 224. The scale marked on the photographs is $1^{\circ}$. The tick marks on the infrared maps are spaced by 21:7. (Optical photographs reproduced by permission of the California Institute of Technology. () 1960 National Geographic Society-Palomar Sky Survey.)

Rice et al. $($ see 68,102$)$ 


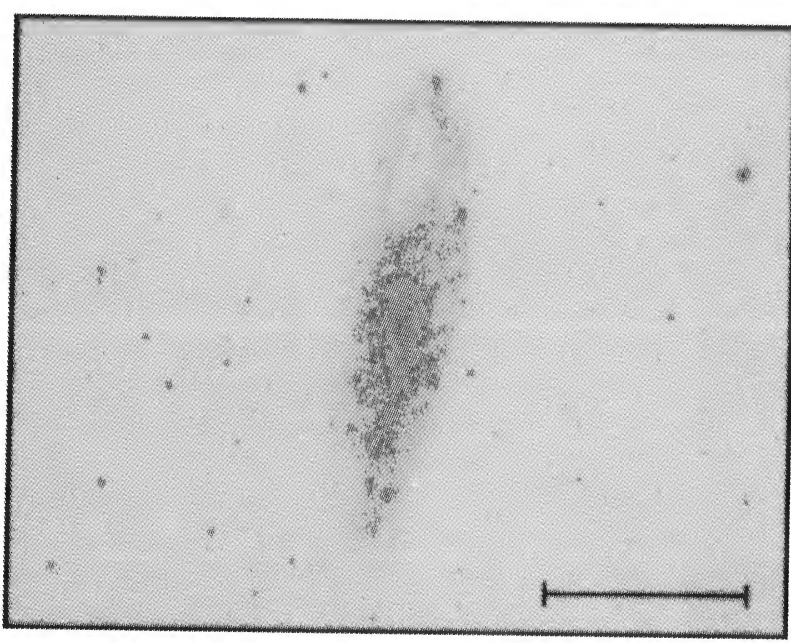

Blue Light
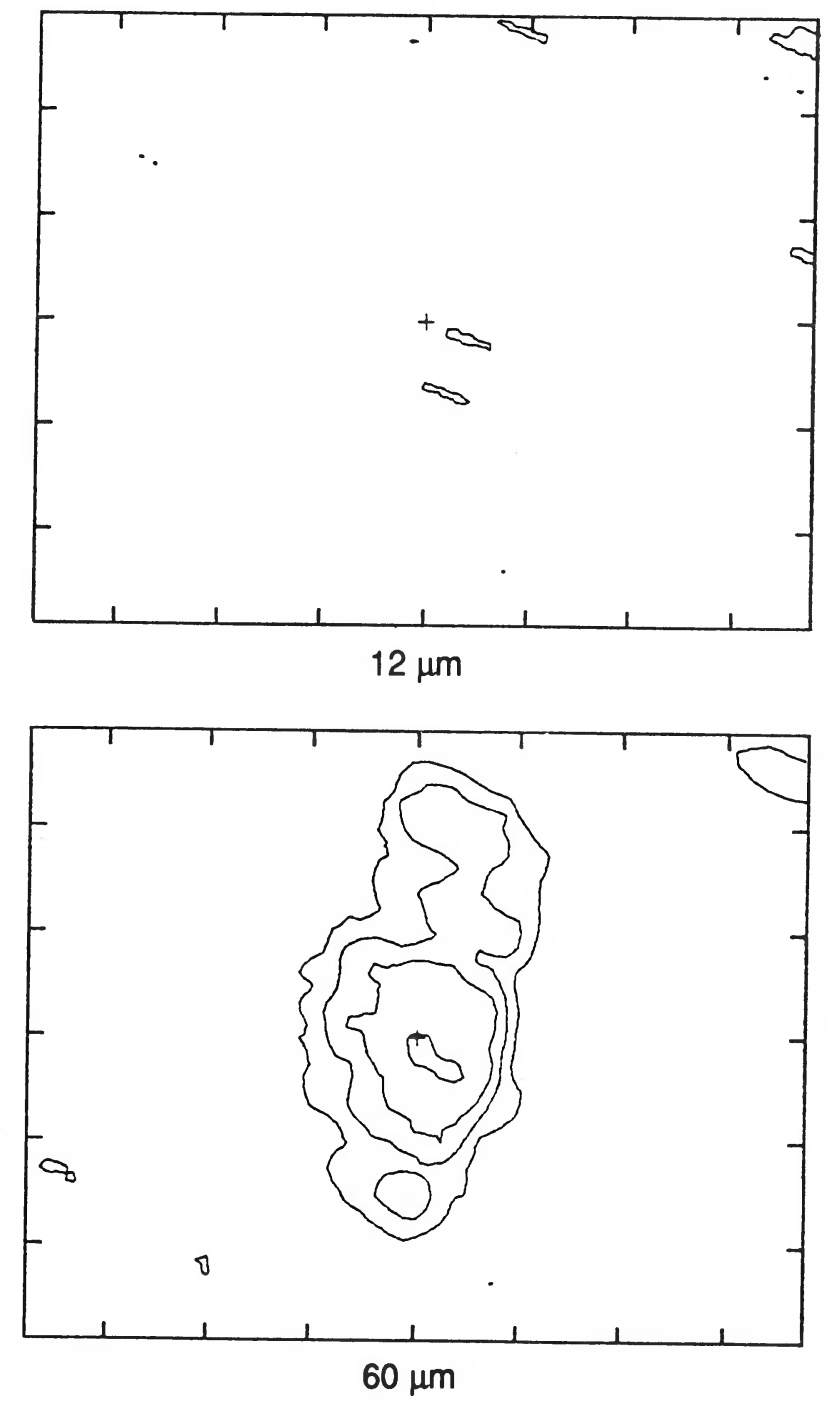

Blue and $60 \mu \mathrm{m}$
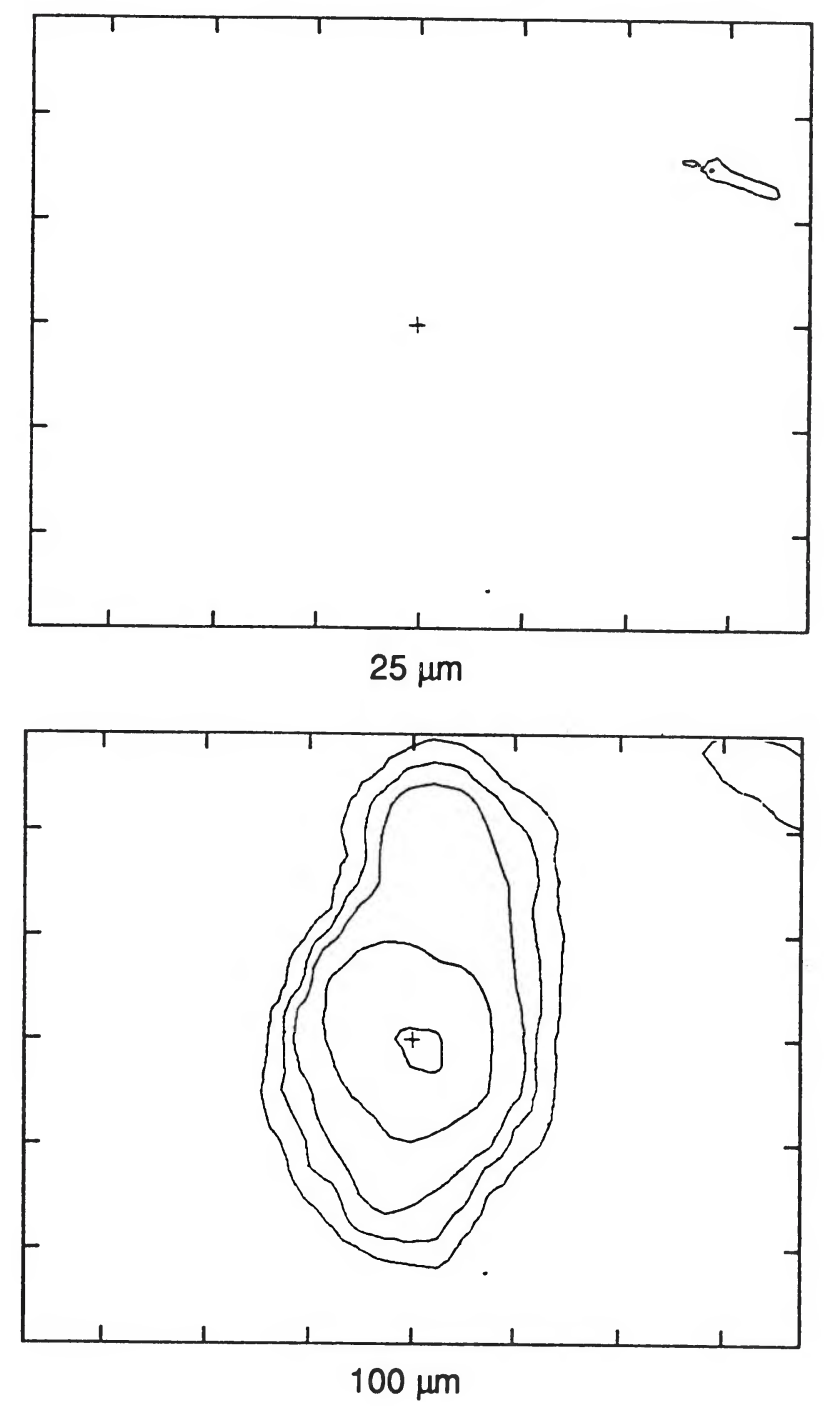

FIG. 4.-NGC 247. (Optical photographs reproduced by permission of the European Southern Observatory.)

RicE et al. (see 68, 102) 


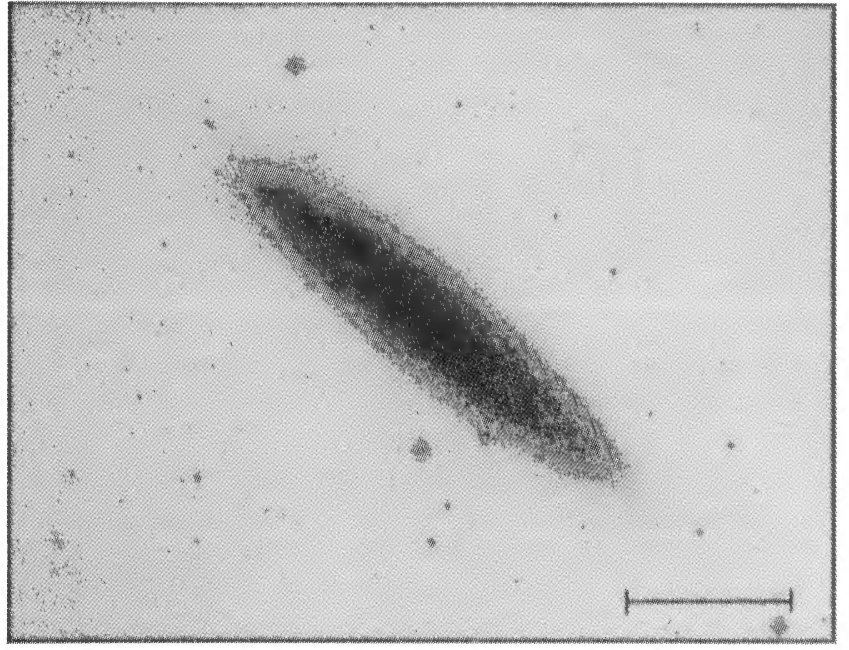

Blue Light
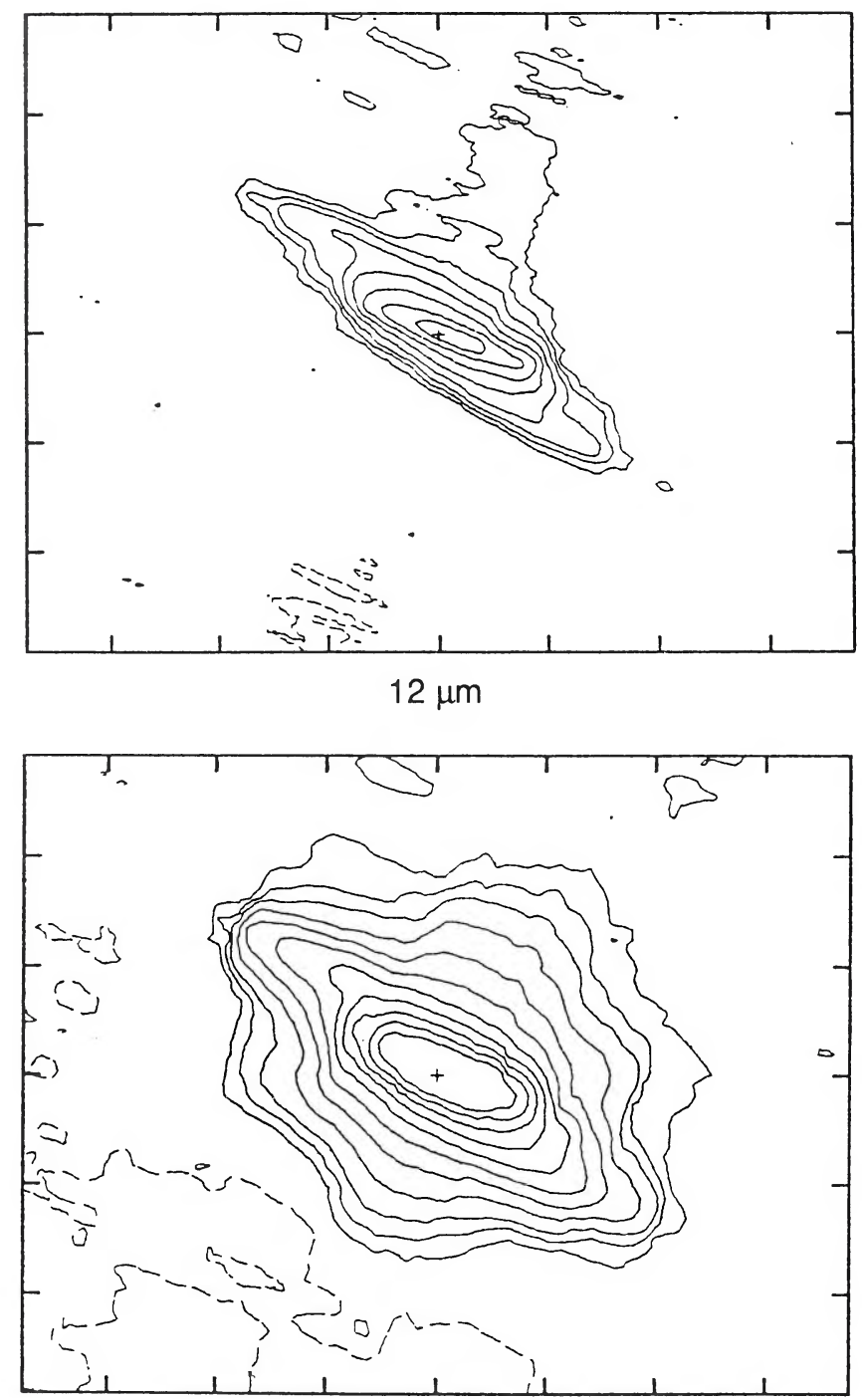

$60 \mu \mathrm{m}$

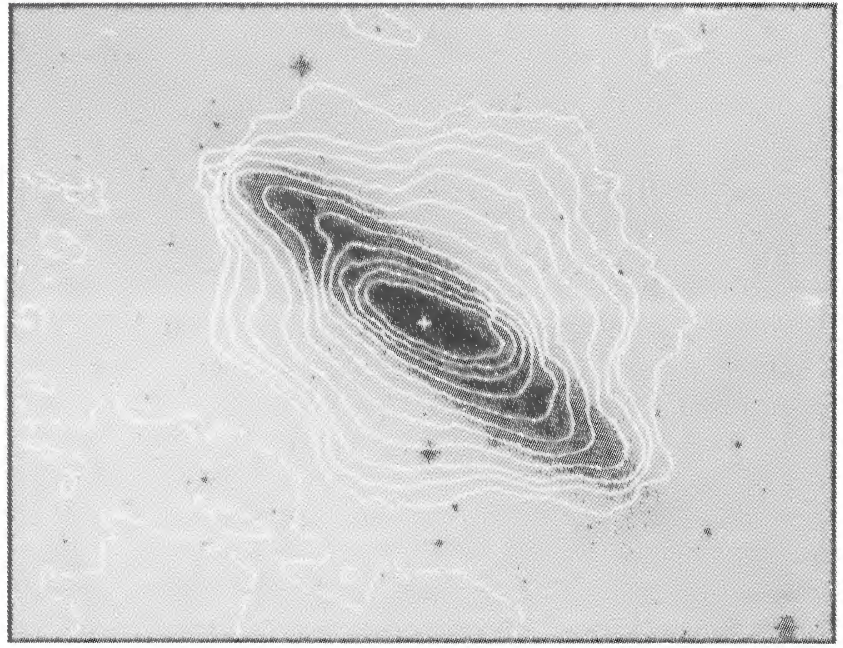

Blue and $60 \mu \mathrm{m}$

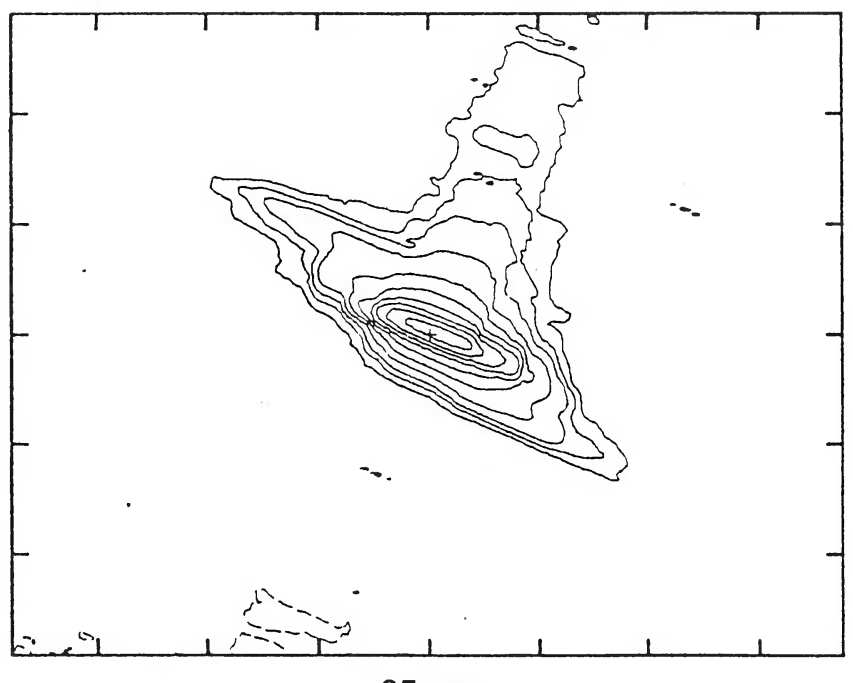

$25 \mu \mathrm{m}$

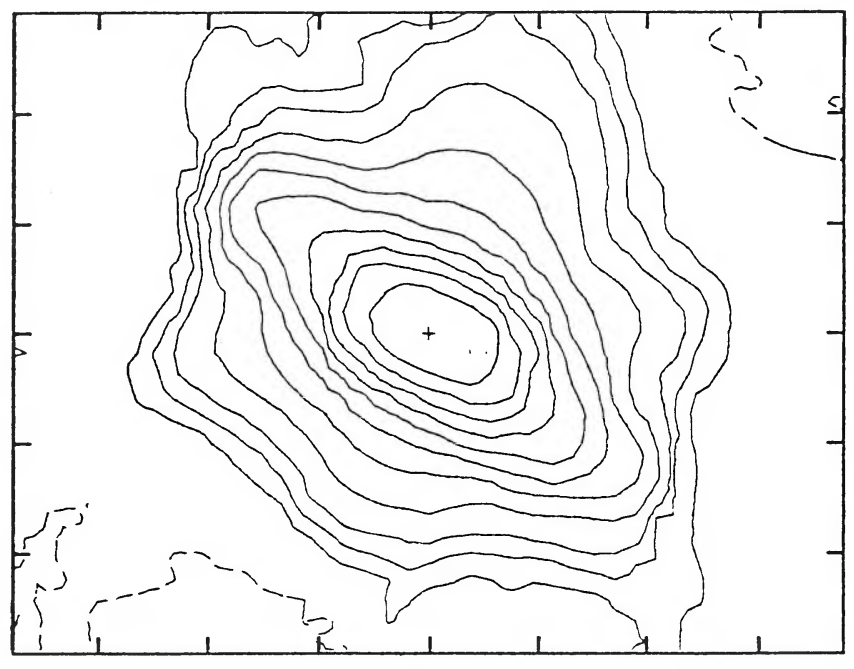

$100 \mu \mathrm{m}$

FIG. 5.- NGC 253. The tick marks on the infrared maps are spaced by 5.4. (Optical photographs reproduced by permission of the European Southern Observatory.)

Rice et al. (see 68, 102) 


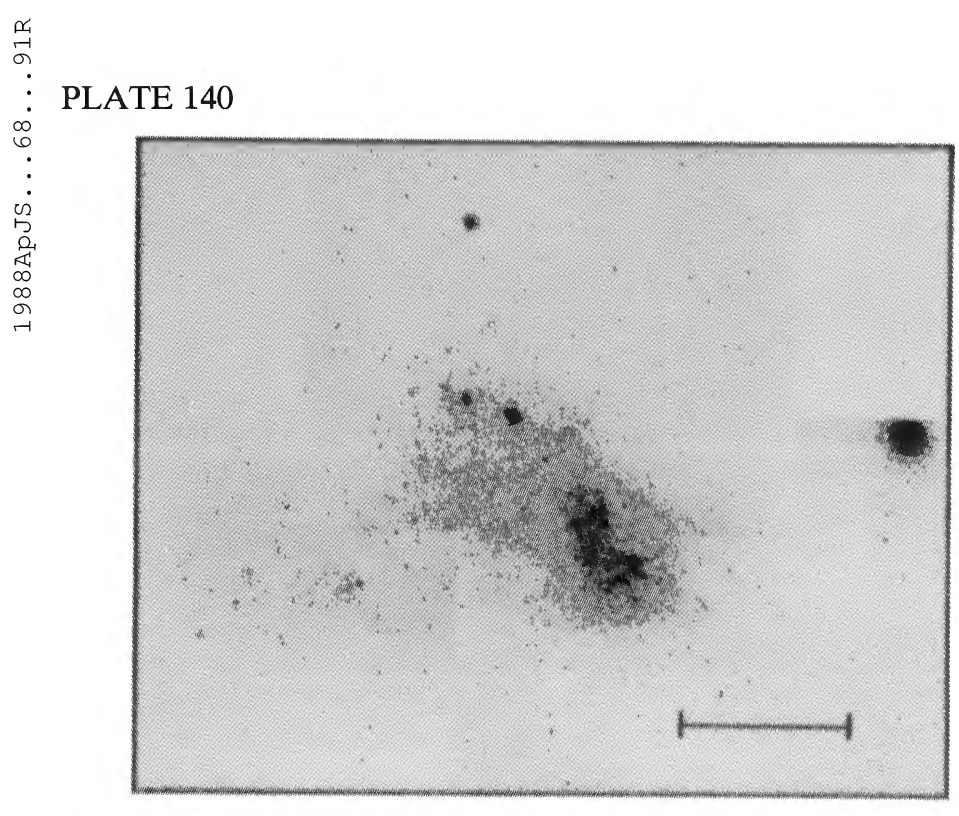

Red Light

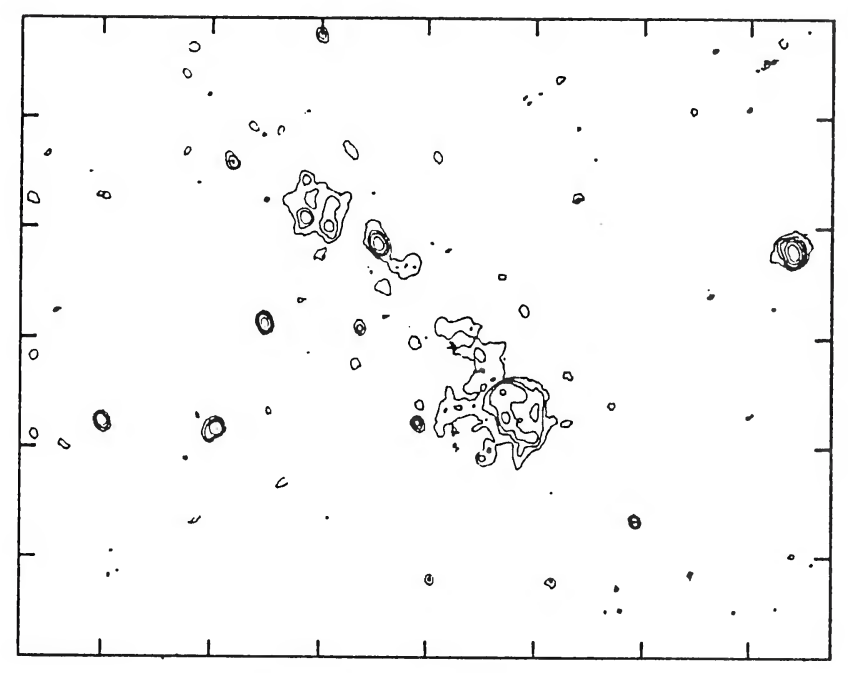

$12 \mu \mathrm{m}$

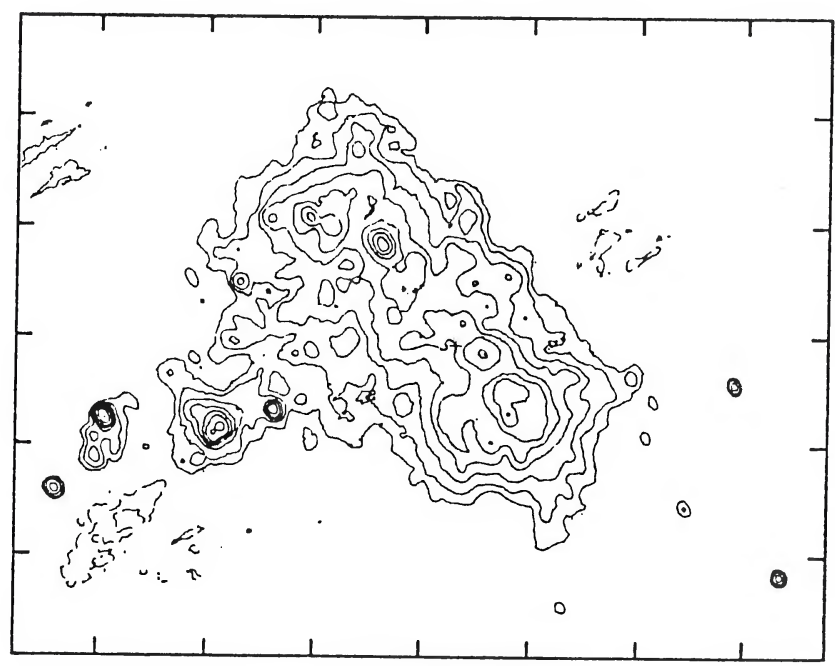

$60 \mu \mathrm{m}$

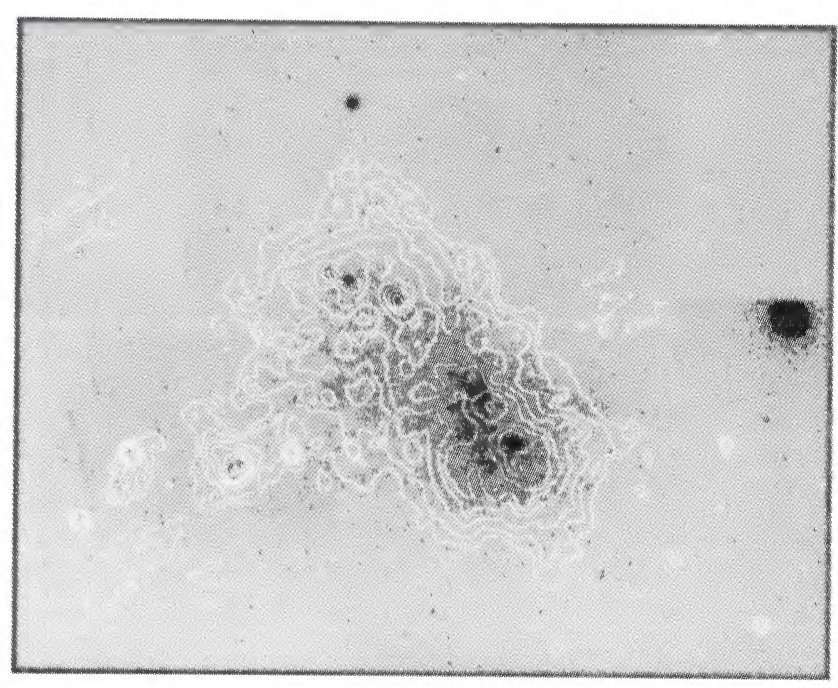

Red and $60 \mu \mathrm{m}$

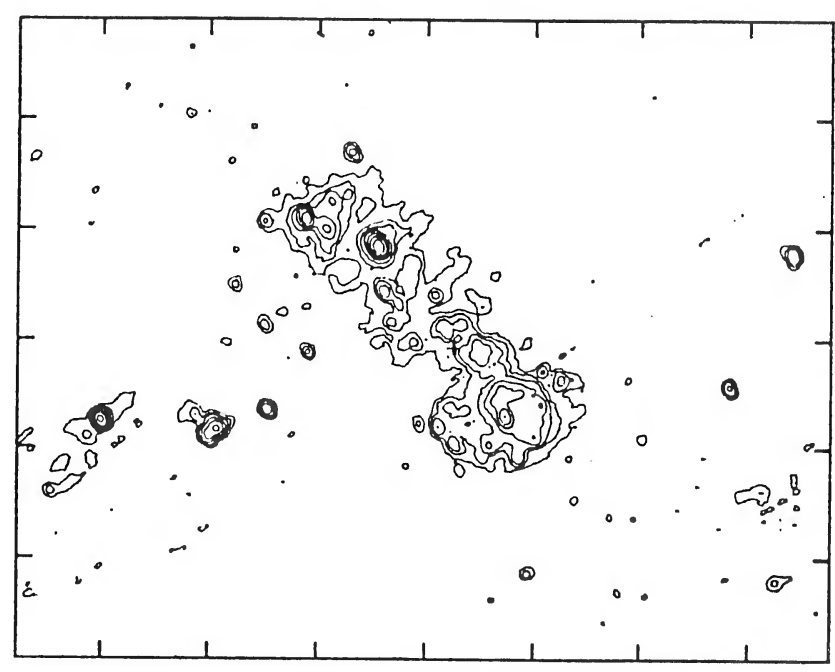

$25 \mu \mathrm{m}$

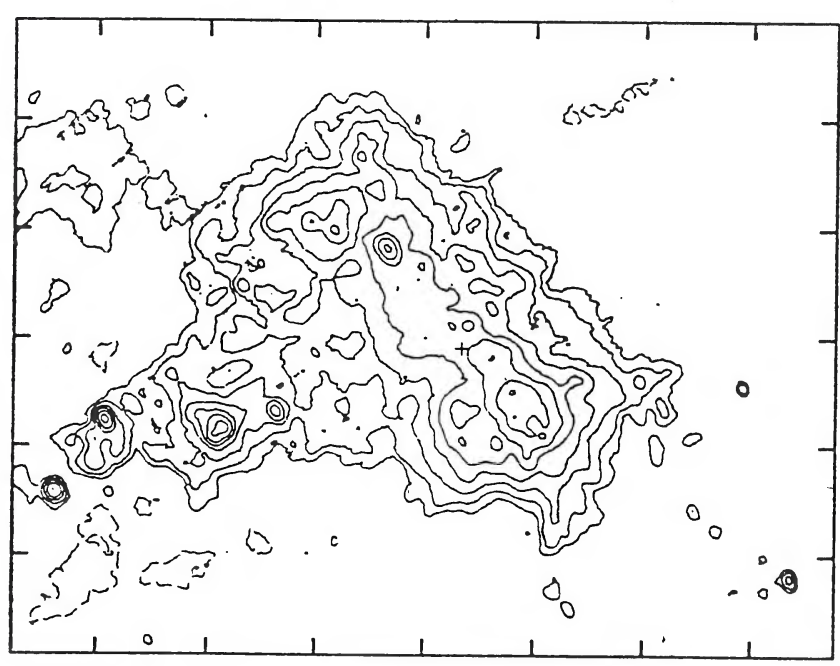

$100 \mu \mathrm{m}$

FIG. 6.-SMC. The scale marked on the photograph is $1^{\circ}$. The tick marks on the infrared map are spaced by $42^{\prime}$. The 12,25 , and $60 \mu \mathrm{m}$ maps have been smoothed to the resolution of the $100 \mu \mathrm{m}$ map. The contour levels of the 60 and $100 \mu \mathrm{m}$ maps are at $($ dashed $) 9 \sigma$ below the map mean background level and at (solid) $10 \sigma$ and with brighter contours scaled logarithmically by 2 from $10 \sigma$. (Optical photographs reproduced by permission of the Mount Wilson and Las Campanas Observatories, Carnegie Institution of Washington.) 


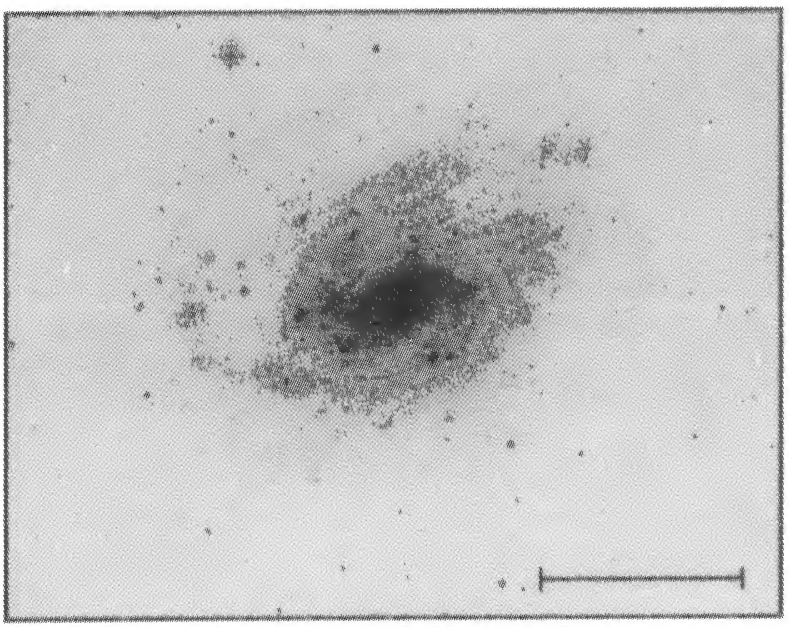

Blue Light

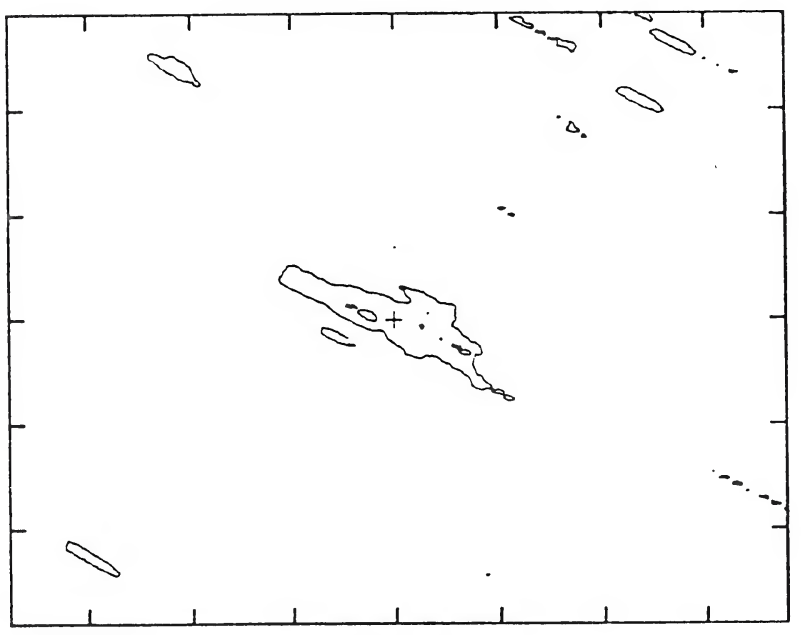

$12 \mu \mathrm{m}$

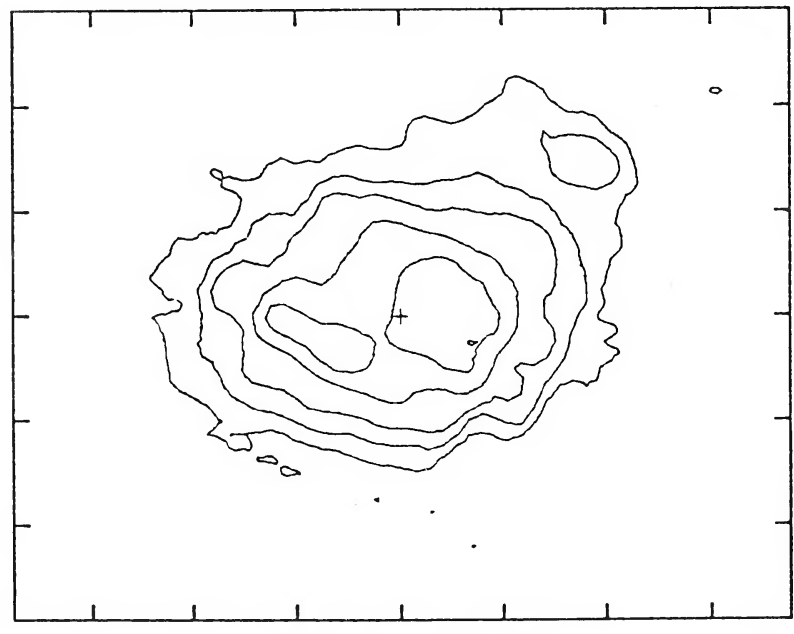

$60 \mu \mathrm{m}$

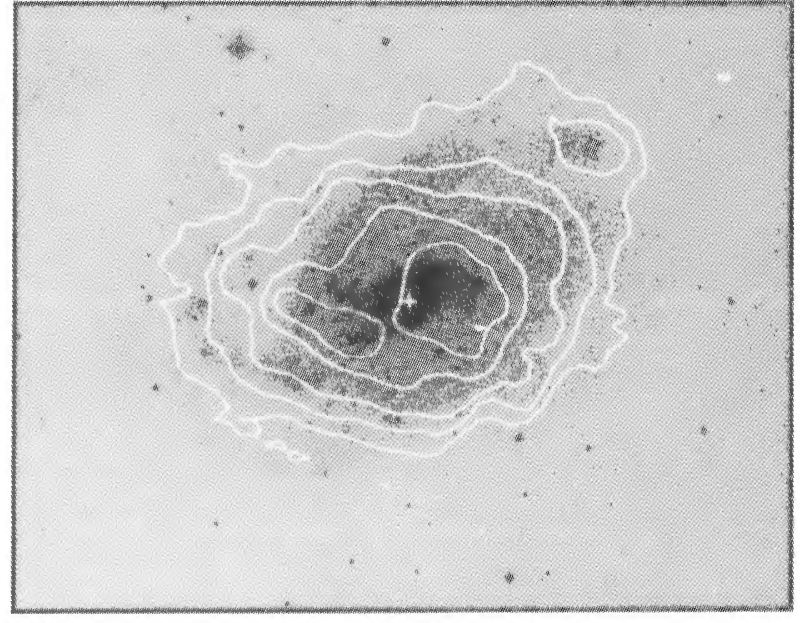

Blue and $60 \mu \mathrm{m}$

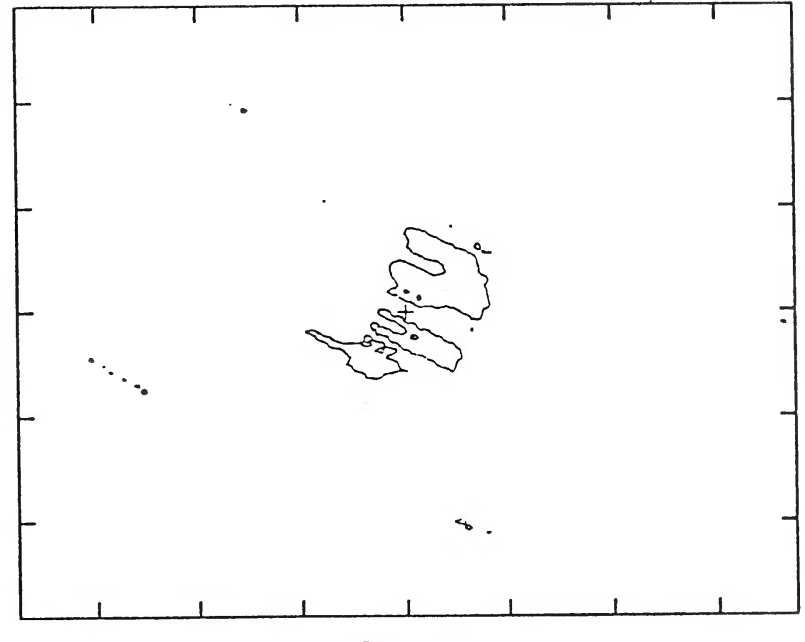

$25 \mu \mathrm{m}$

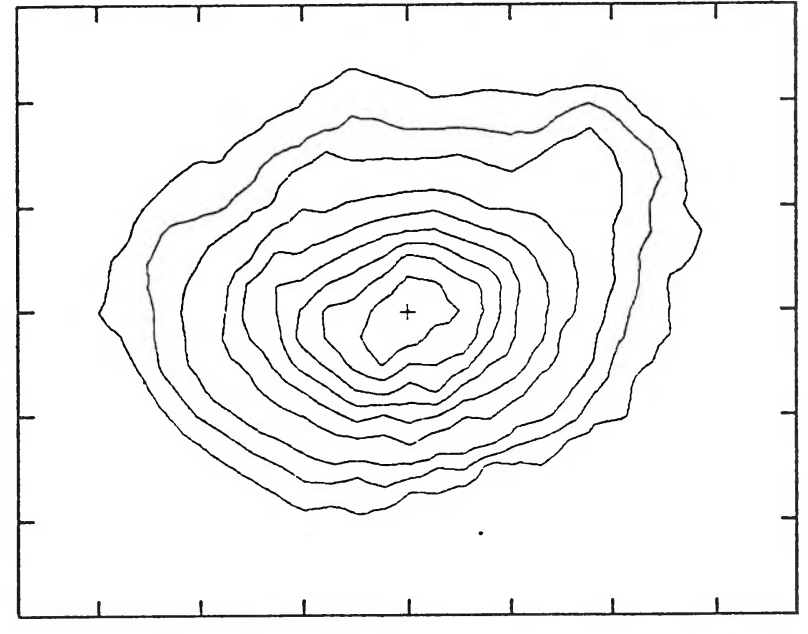

$100 \mu \mathrm{m}$

FIG. 7.-NGC 300. The map contour levels are at (dashed) 3,6, and $9 \sigma$ below the map mean background level and at (solid) 3, 6, and $10 \sigma$ with brighter levels at increments of $10 \sigma$. (Optical photographs reproduced by permission of the European Southern Observatory.)

RICE et al. (see 68, 102) 


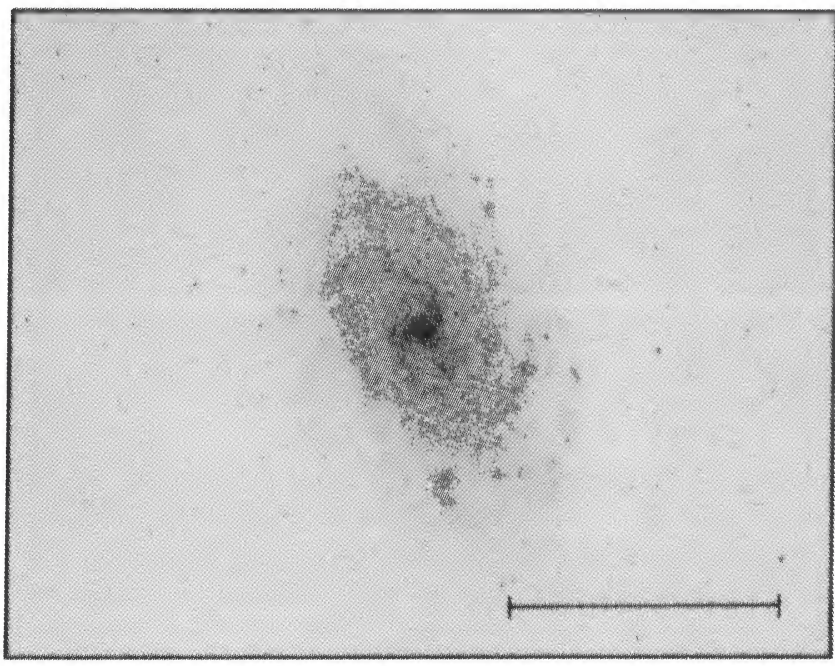

Blue Light

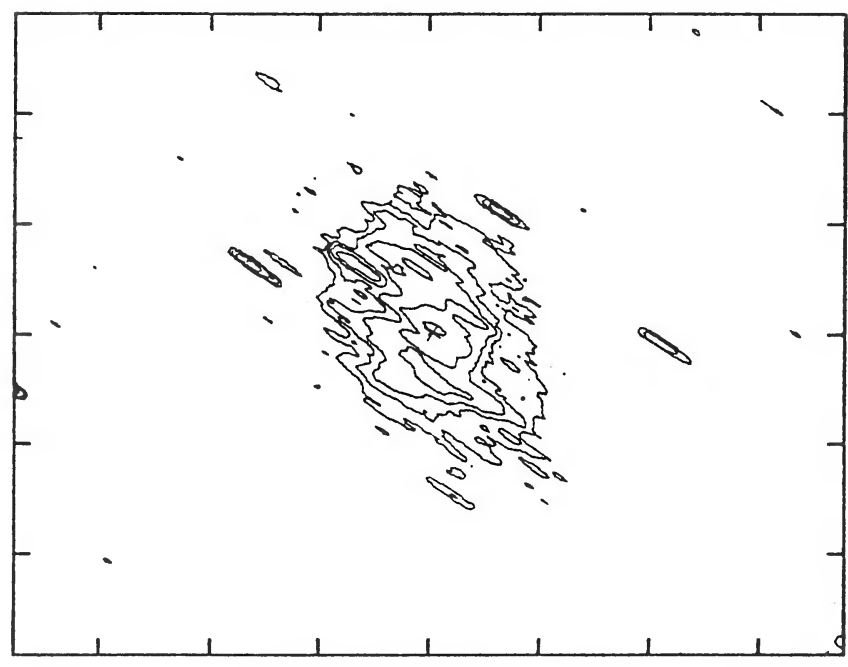

$12 \mu \mathrm{m}$

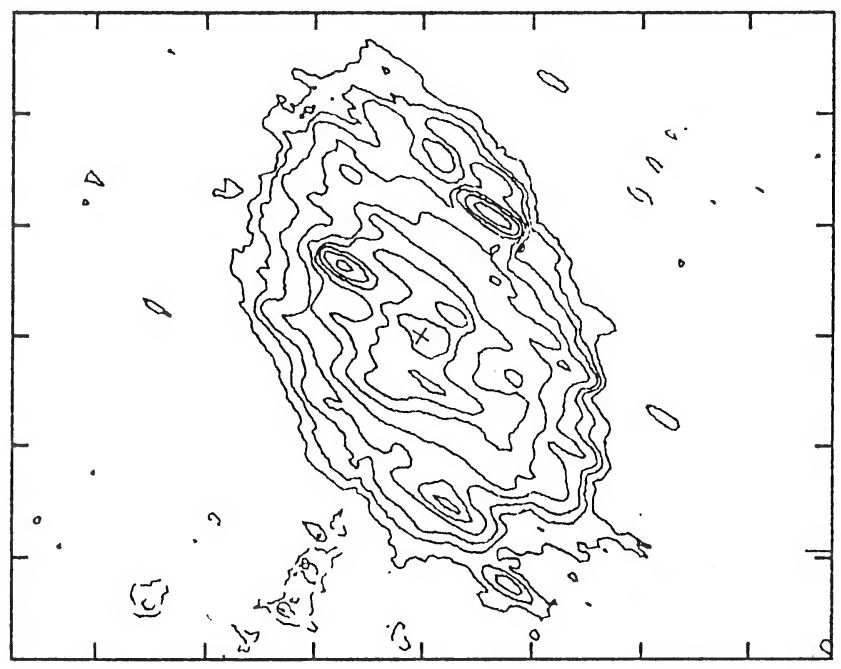

$60 \mu \mathrm{m}$

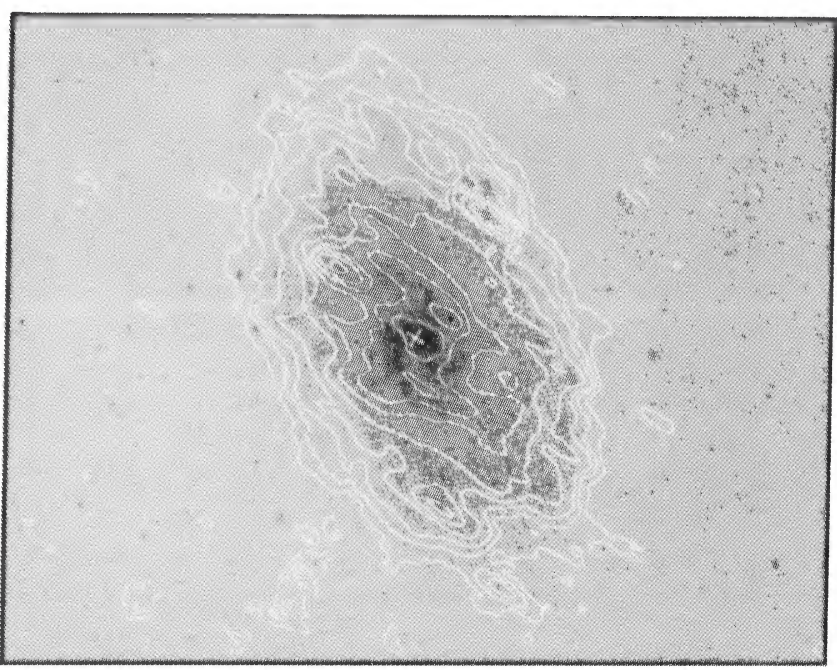

Blue and $60 \mu \mathrm{m}$

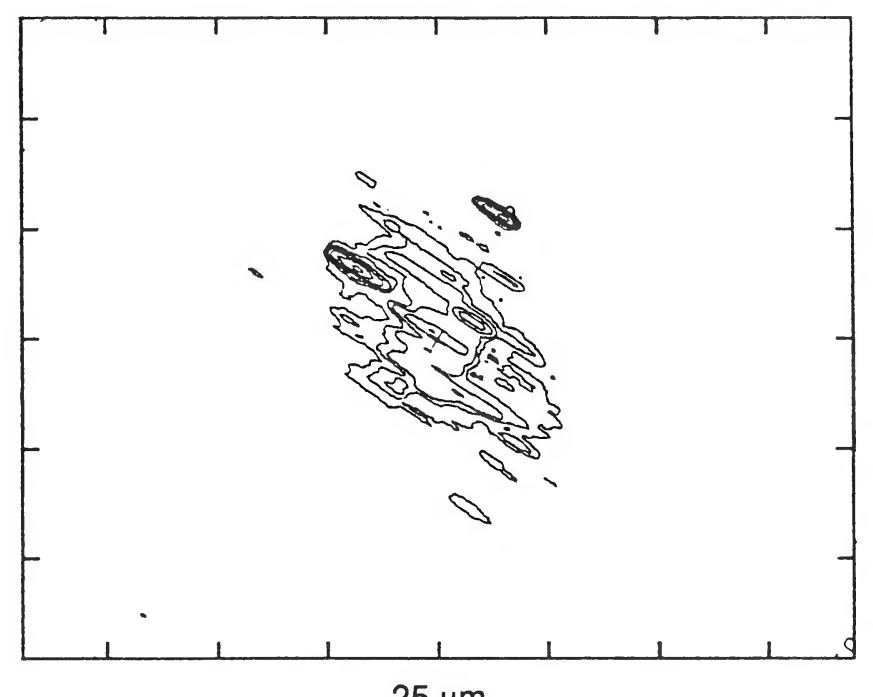

$25 \mu \mathrm{m}$

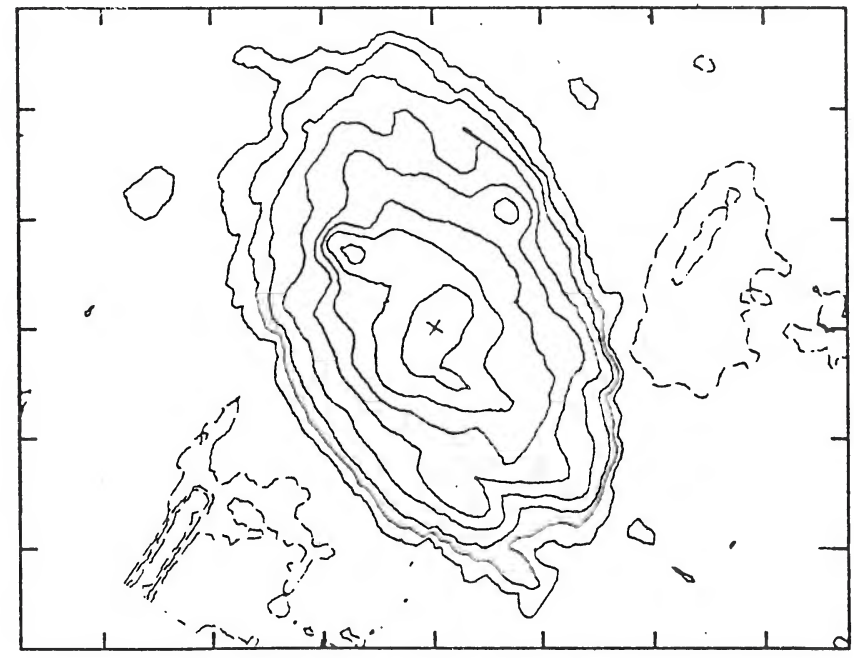

$100 \mu \mathrm{m}$

FIG. 8. - NGC 598. The scale marked on the photographs is $30^{\prime}$. The tick marks on the infrared maps are spaced by 12.4 . (Optical photographs reproduced by permission of the California Institute of Technology. (C) 1960 National Geographic Society-Palomar Sky Survey.)

RICE et al. (see 68, 102) 


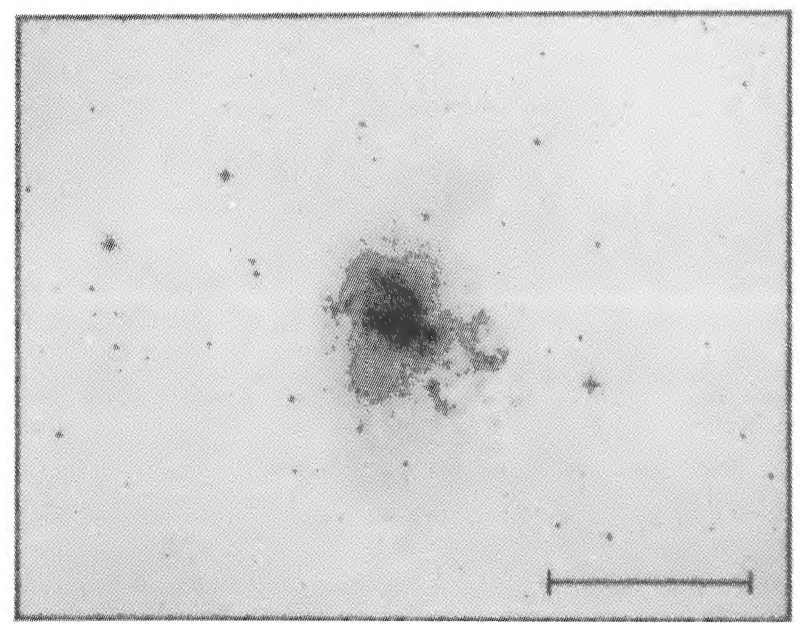

Blue Light

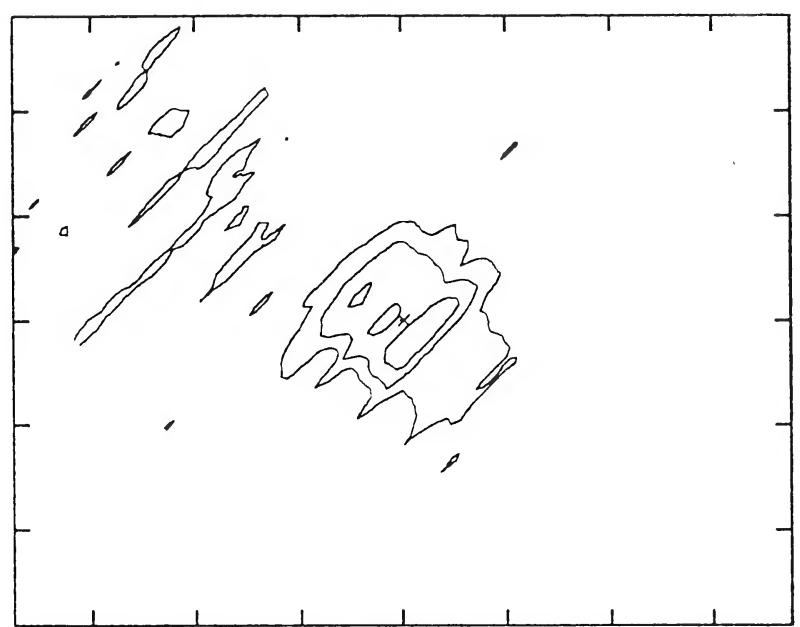

$12 \mu \mathrm{m}$

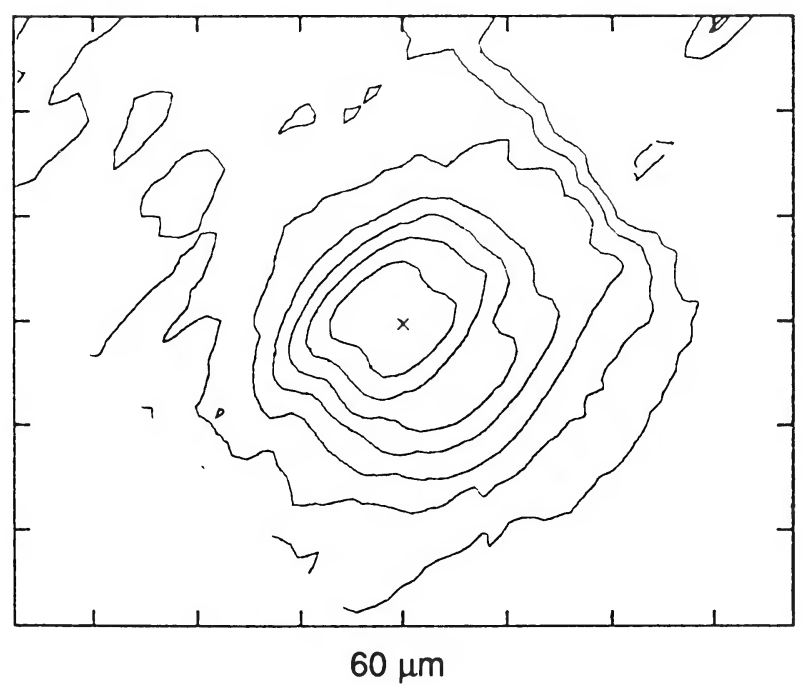

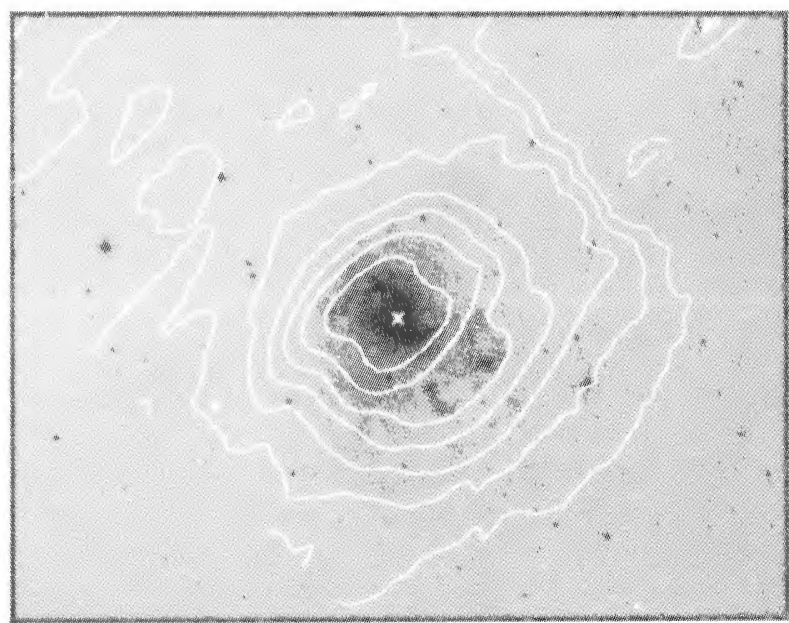

Blue and $60 \mu \mathrm{m}$
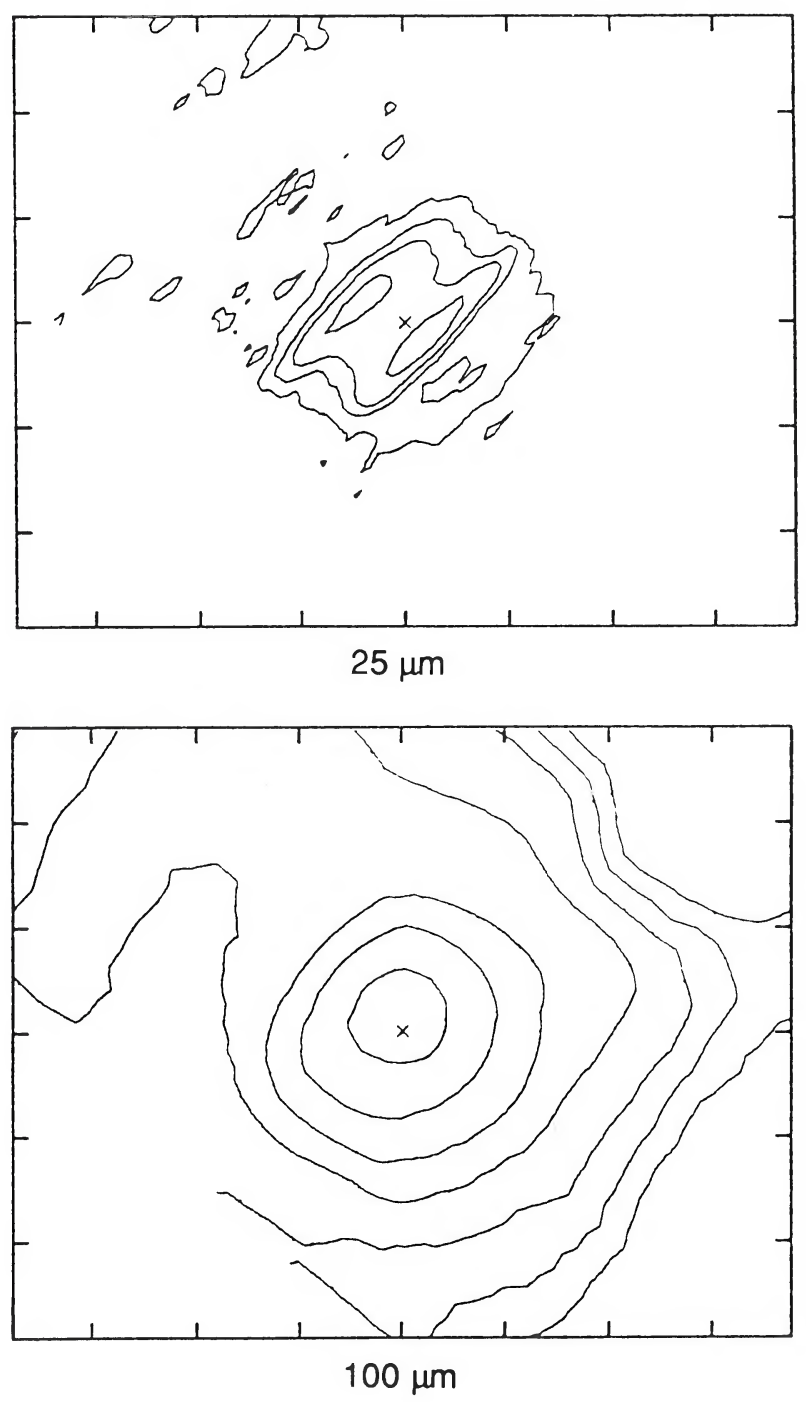

FIG. 9.-NGC 1313. (Optical photographs reproduced by permission of the European Southern Observatory.) RICE et al. (see 68, 102) 


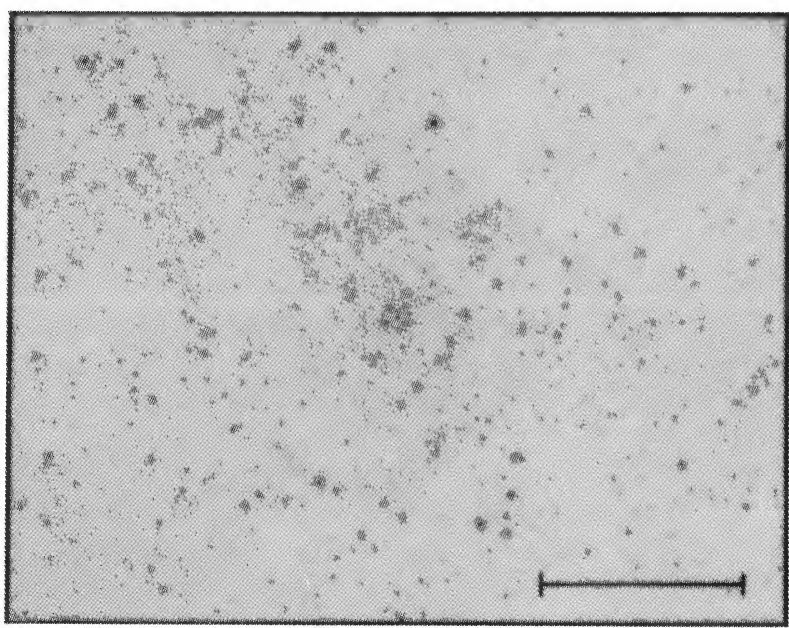

Blue Light
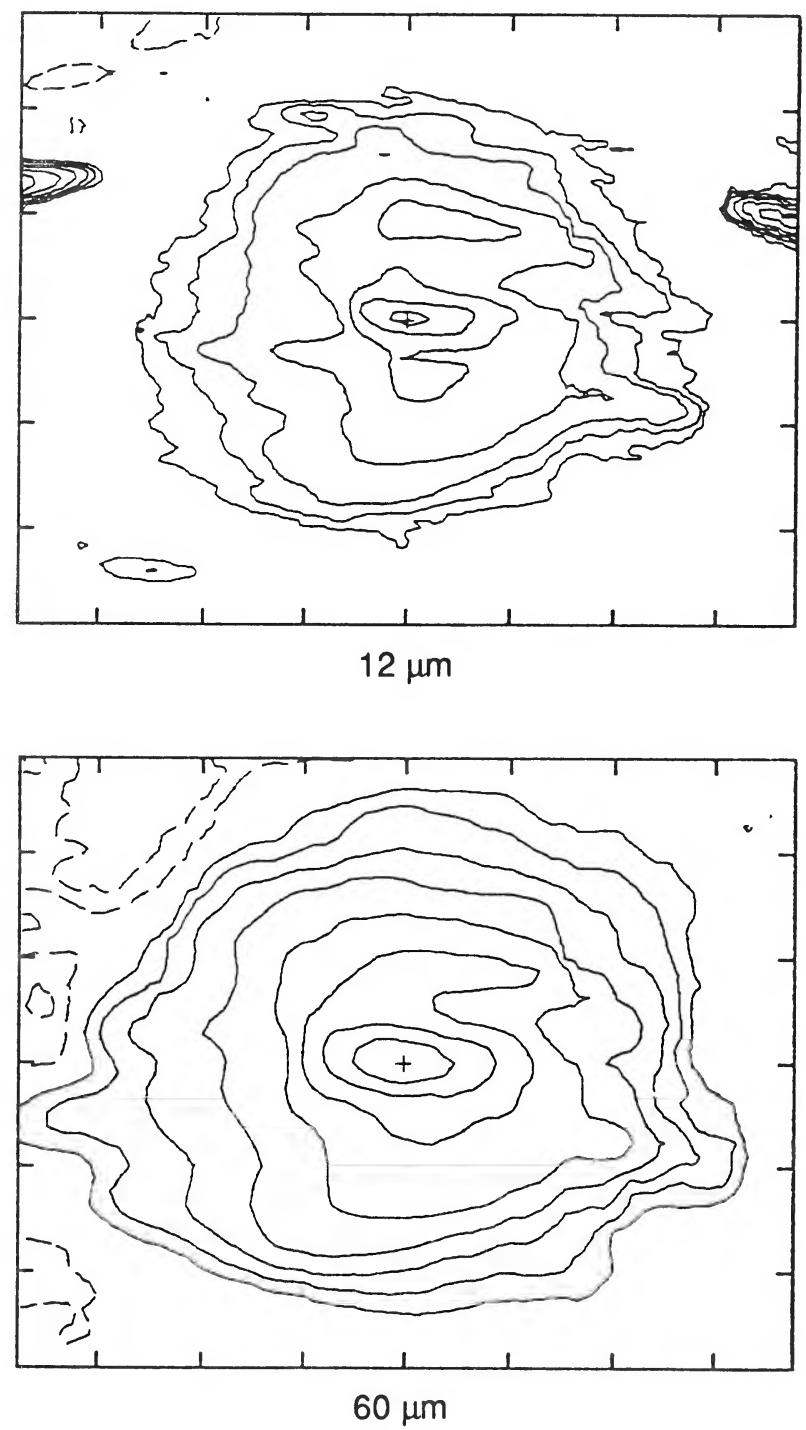

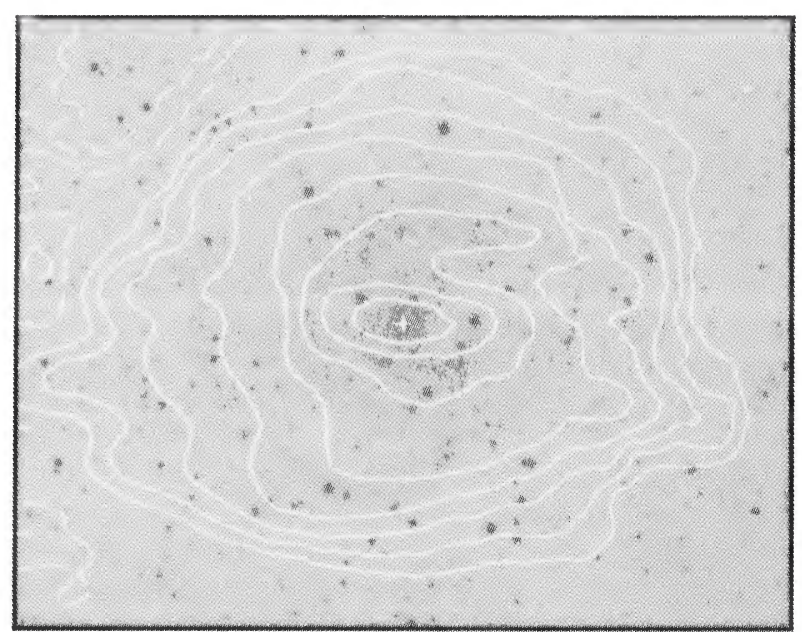

Blue and $60 \mu \mathrm{m}$

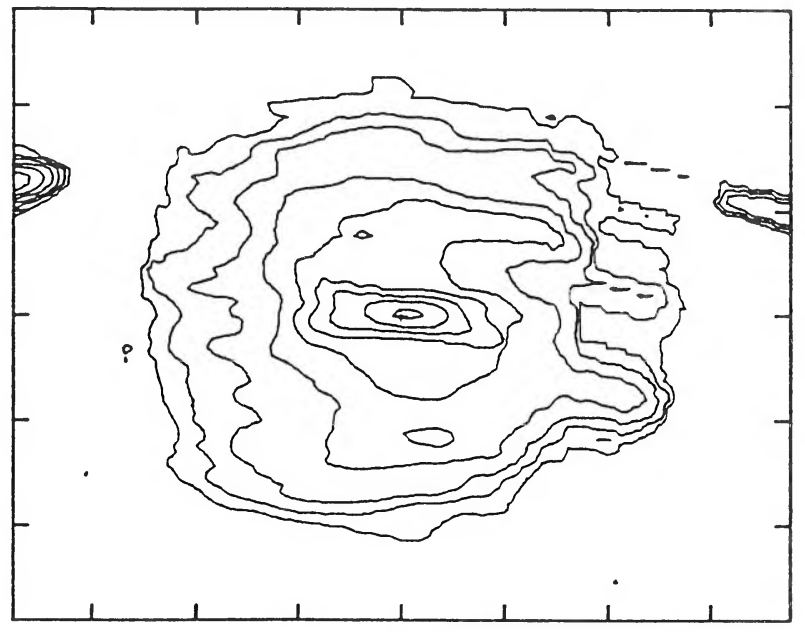

$25 \mu \mathrm{m}$

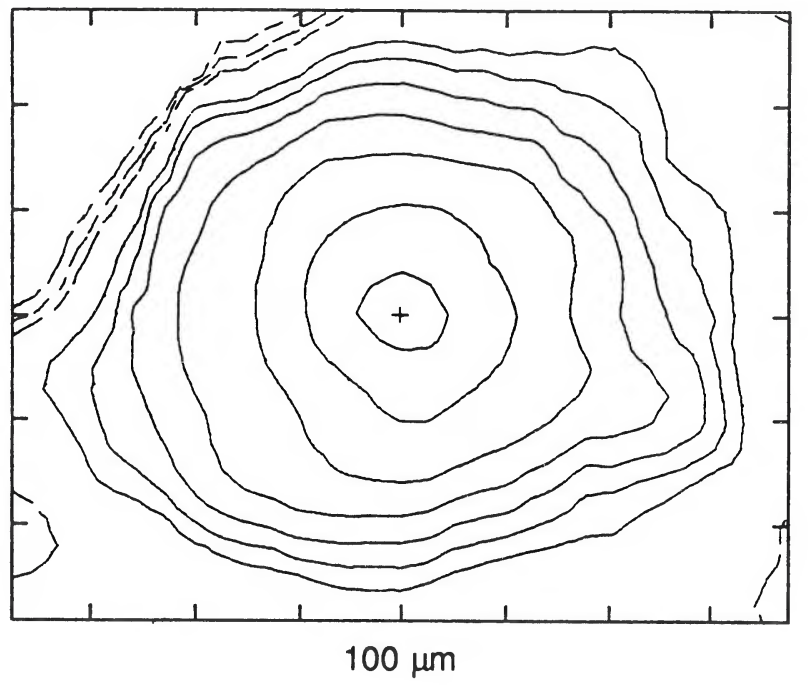

FIG. 10.-IC 342. The solid contour levels of the 60 and $100 \mu \mathrm{m}$ maps are at (dashed) 3, 6, and $9 \sigma$ below the map mean background level and at (solid) $10 \sigma$ and with brighter contours scaled logarithmically by 2 from $10 \sigma$. (Optical photographs reproduced by permission of the California Institute of Technology. (C) 1960 National Geographic Society-Palomar Sky Survey.)

RICE et al. (see 68, 102) 


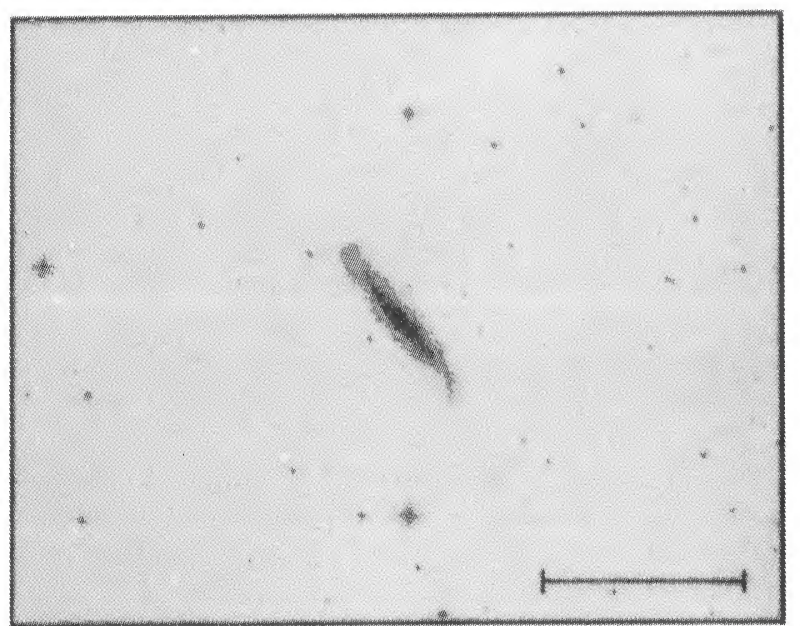

Blue Light

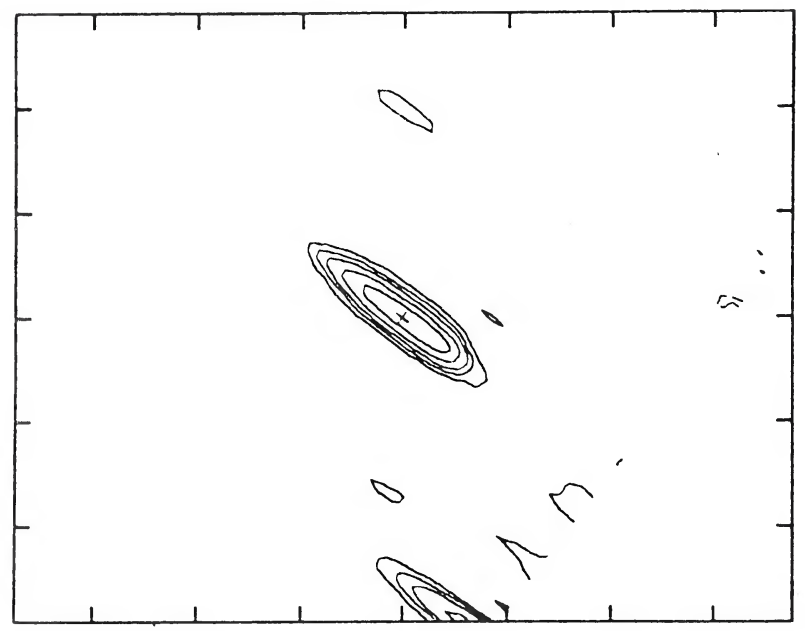

$12 \mu \mathrm{m}$

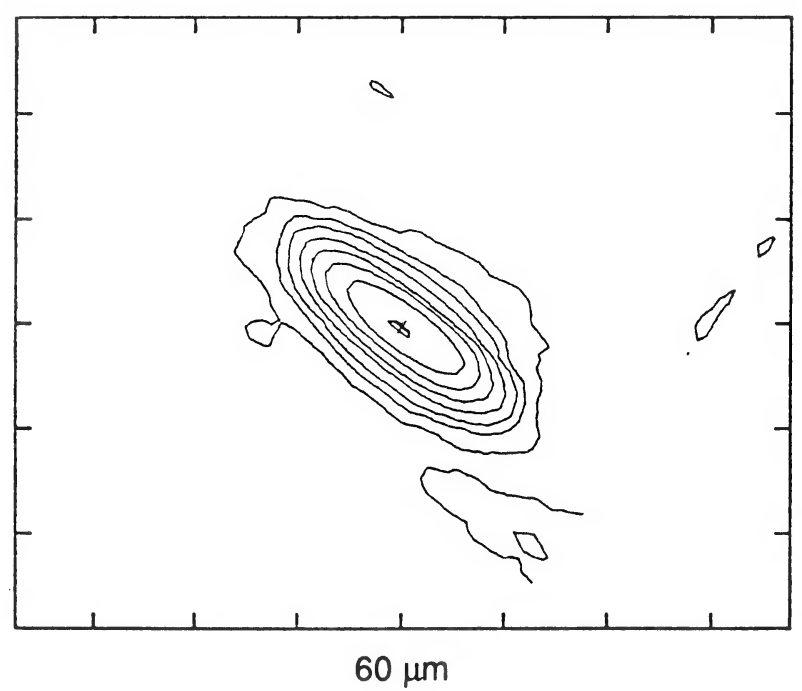

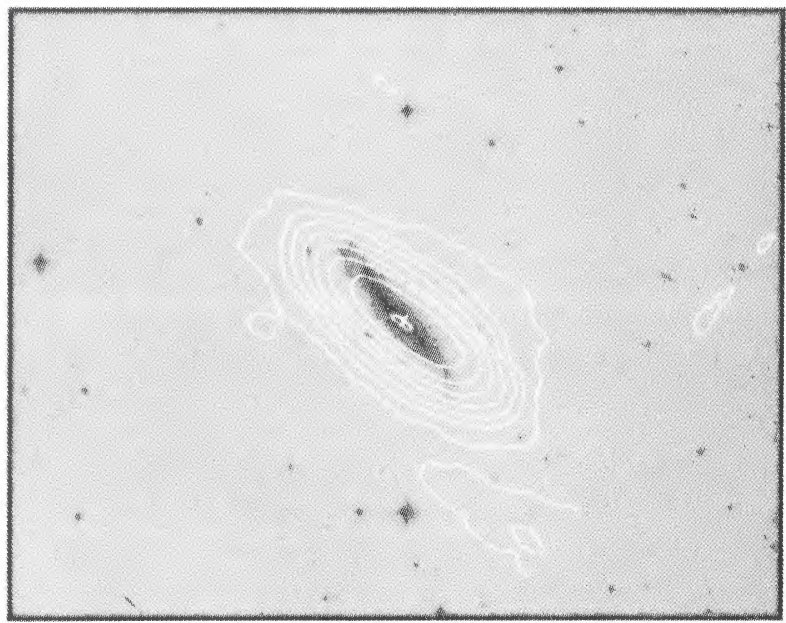

Blue and $60 \mu \mathrm{m}$

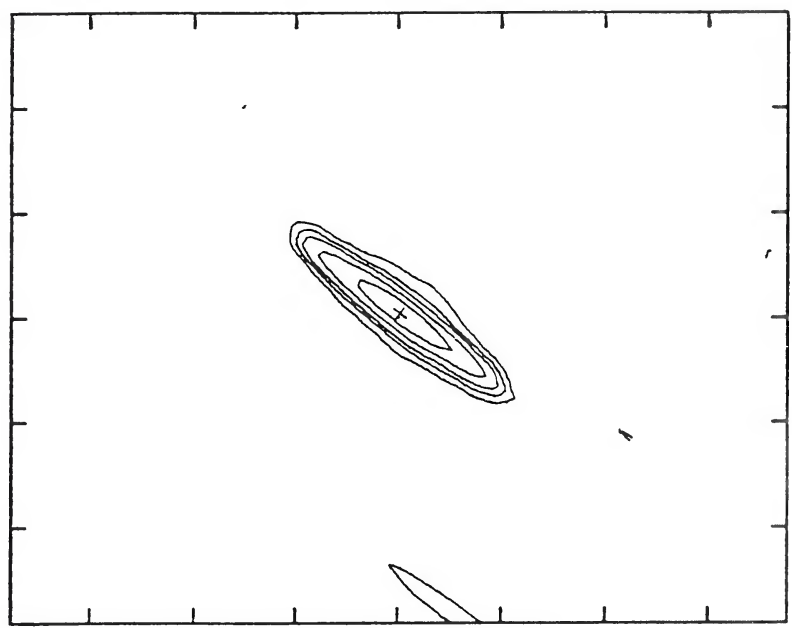

$25 \mu \mathrm{m}$

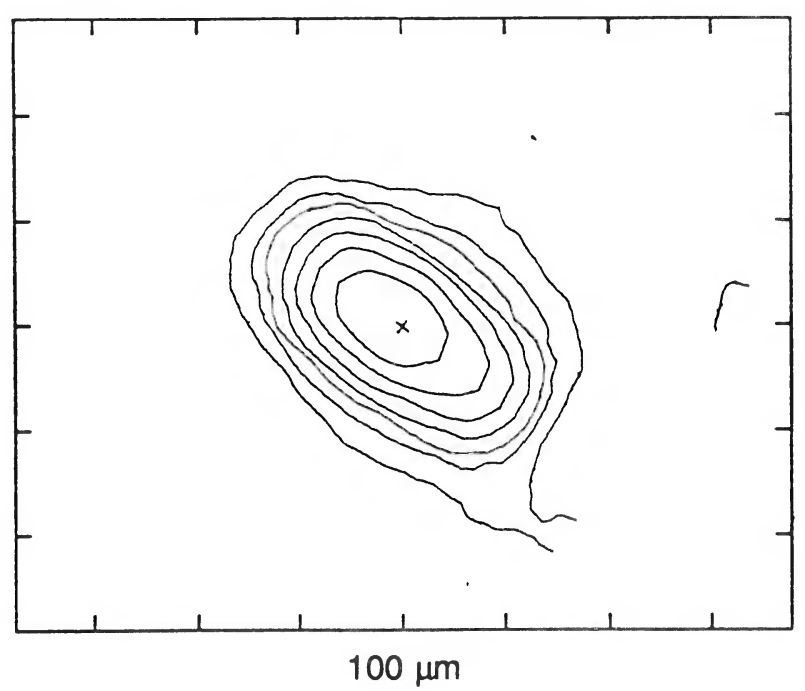

FIG. 11. - NGC 1448. (Optical photographs reproduced by permission of the European Southern Observatory.) RICE et al. (see 68, 102) 


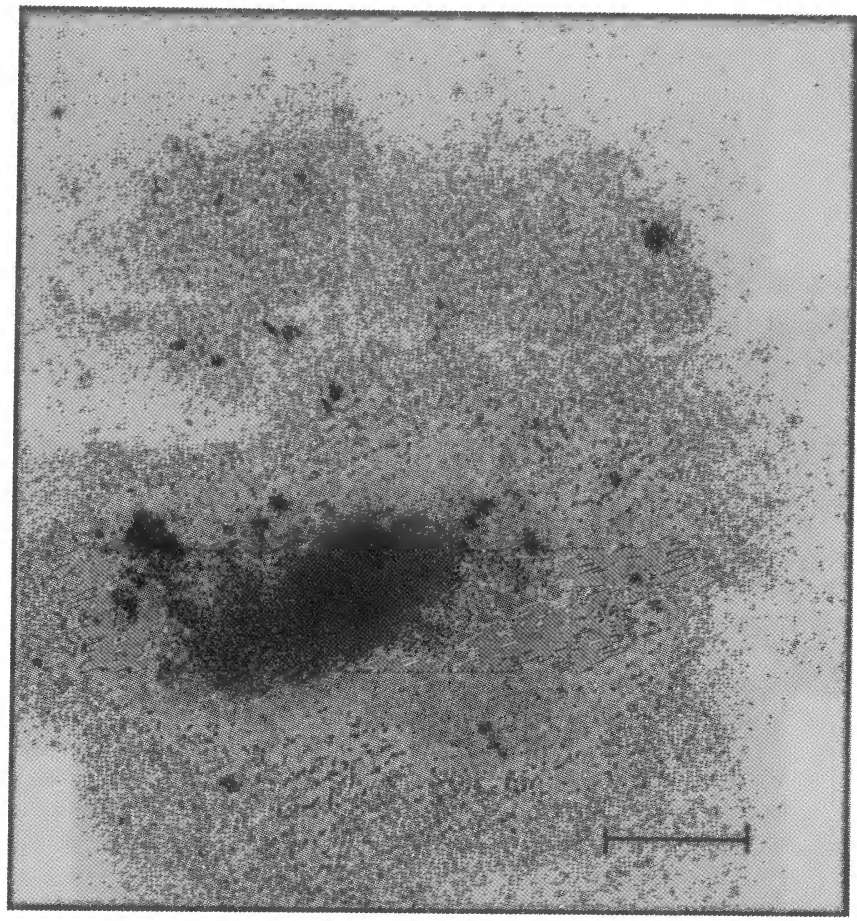

Red Light

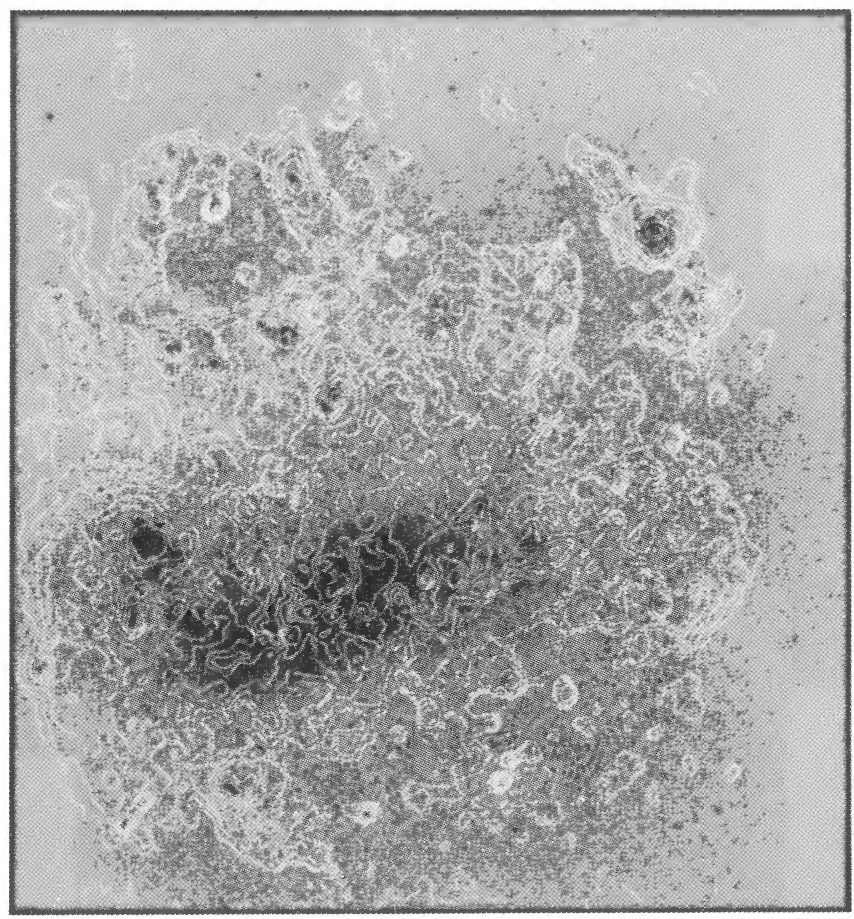

Red and $60 \mu \mathrm{m}$

FIG. 12.-LMC. The scale marked on the photograph is $1^{\circ}$. The tick marks on the infrared map are spaced by $61^{\prime}$. The 12,25 , and $60 \mu \mathrm{m}$ maps have been smoothed to the resolution of the $100 \mu \mathrm{m}$ map. The contour levels of the 60 and $100 \mu \mathrm{m}$ maps are at (dashed) $9 \sigma$ below the map mean background level and at (solid) $10 \sigma$ and with brighter contours scaled logarithmically by 2 from $10 \sigma$. (Optical photographs reproduced by permission of the Mount Wilson and Las Campanas Observatories, Carnegie Institution of Washington.)

Rice et al. (see 68, 102) 
PLATE 147

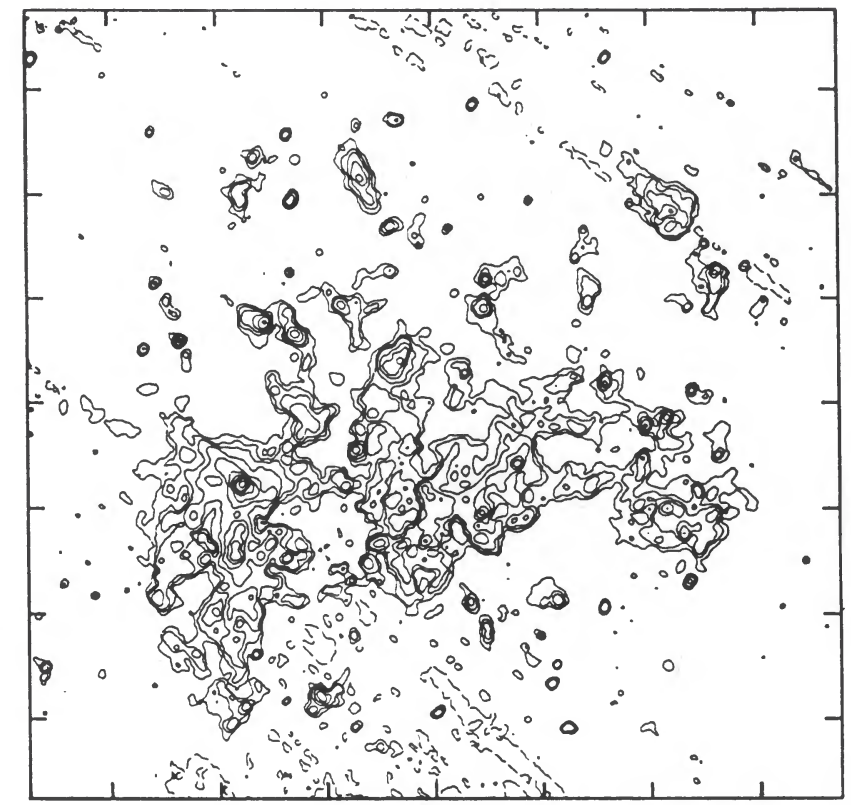

$12 \mu \mathrm{m}$

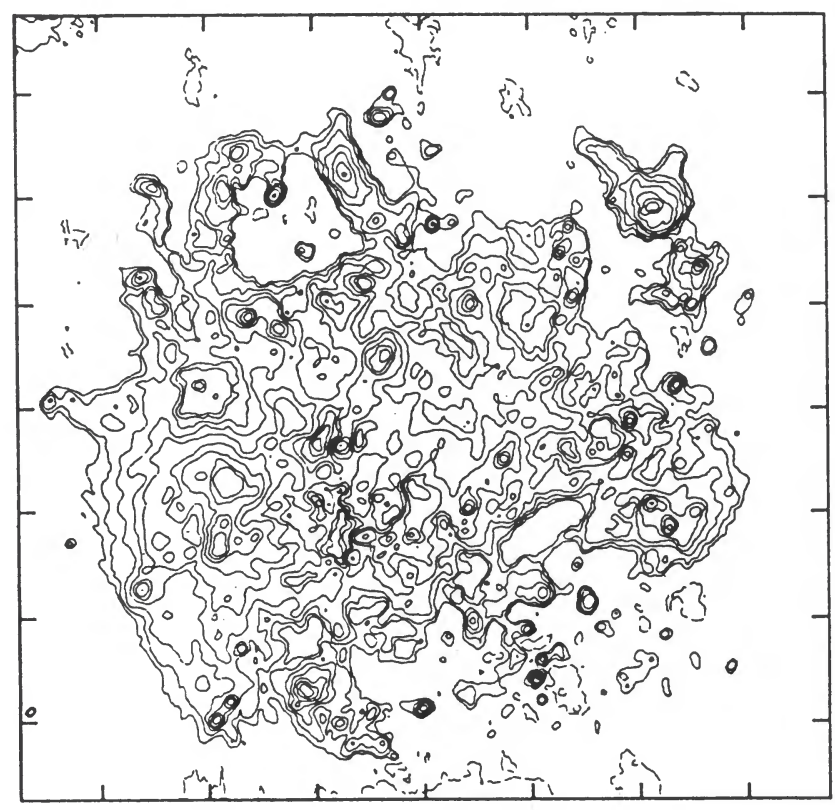

$60 \mu \mathrm{m}$

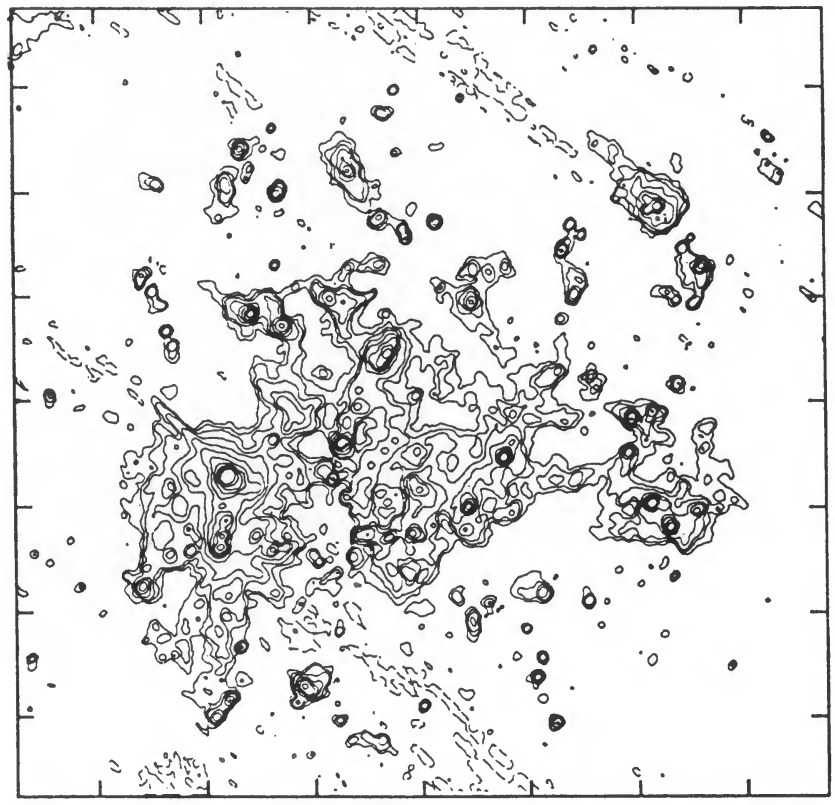

$25 \mu \mathrm{m}$

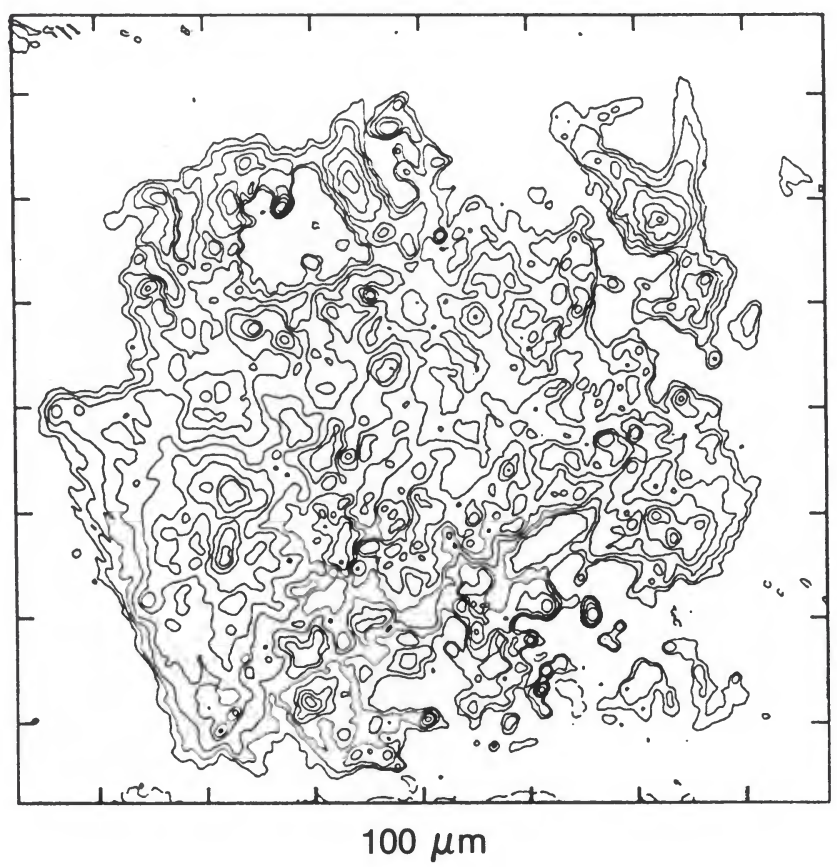

$100 \mu \mathrm{m}$

RICE et al. (see 68, 102)

FIG. 12-Continued 


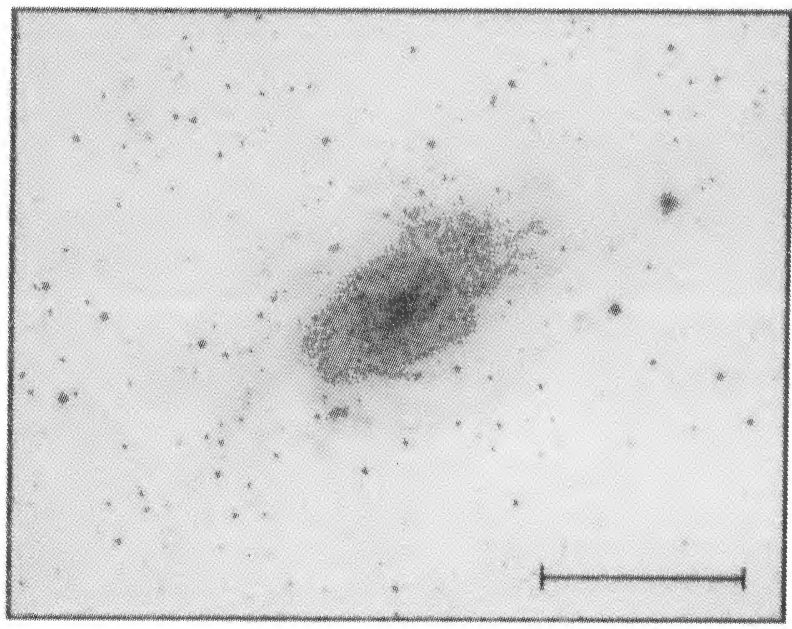

Blue Light
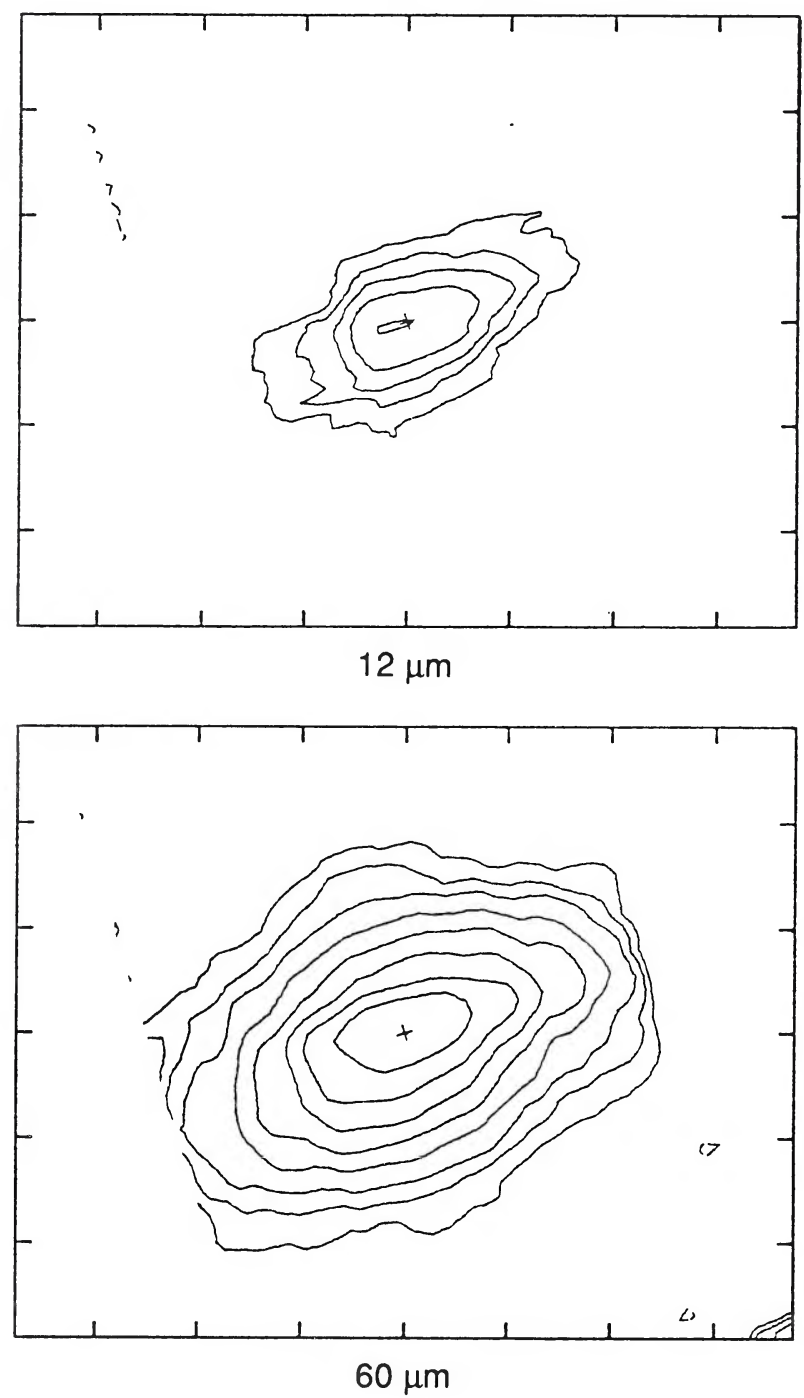

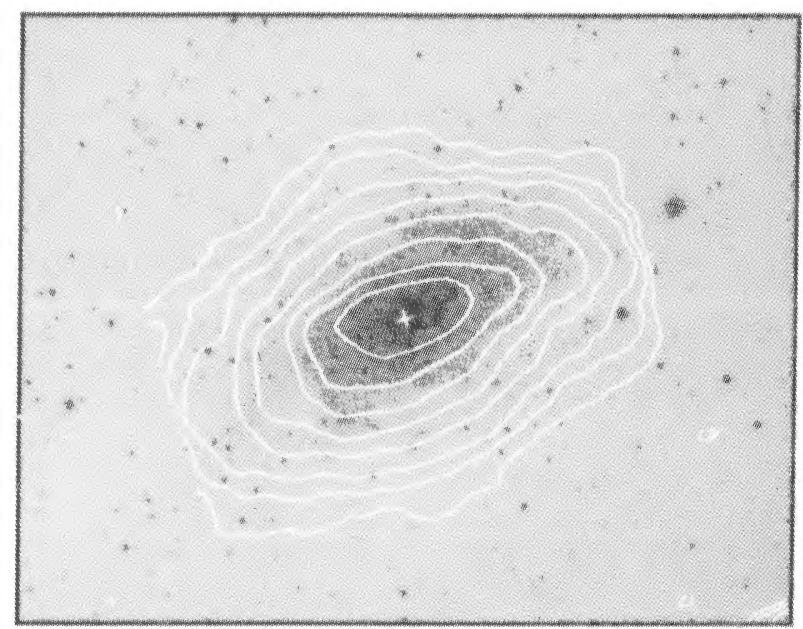

Blue and $60 \mu \mathrm{m}$

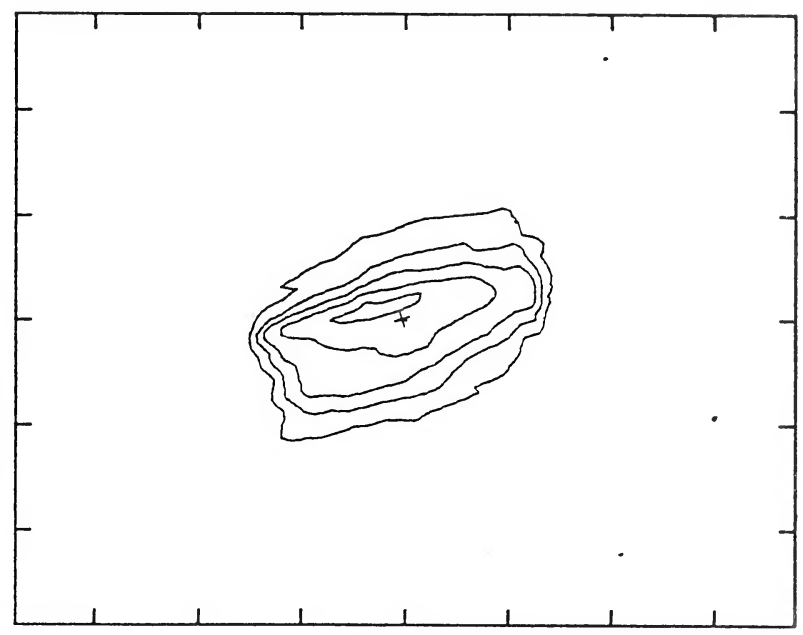

$25 \mu \mathrm{m}$

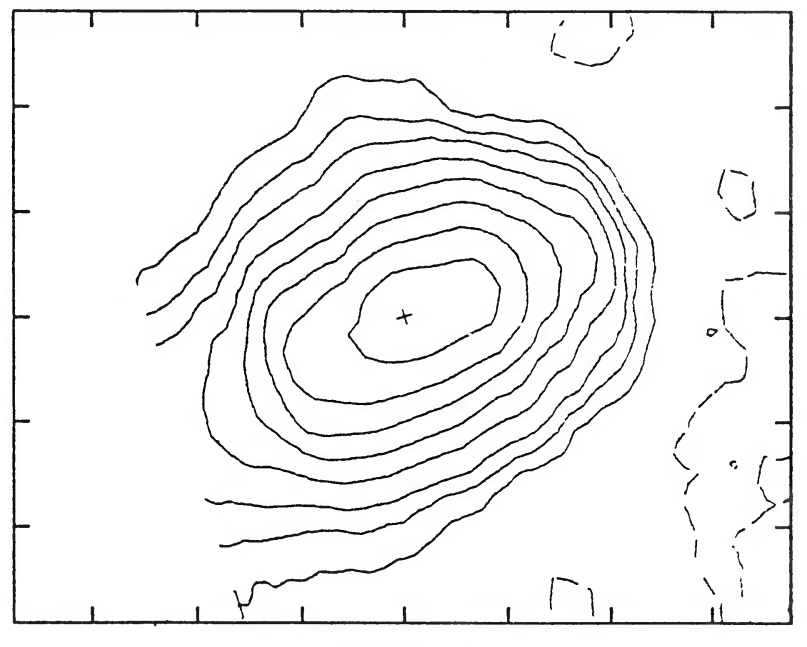

$100 \mu \mathrm{m}$

FIG. 13.-NGC 2403. (Optical photographs reproduced by permission of the California Institute of Technology. (c) 1960 National Geographic Society-Palomar Sky Survey.)

RICE et al. (see 68, 102) 


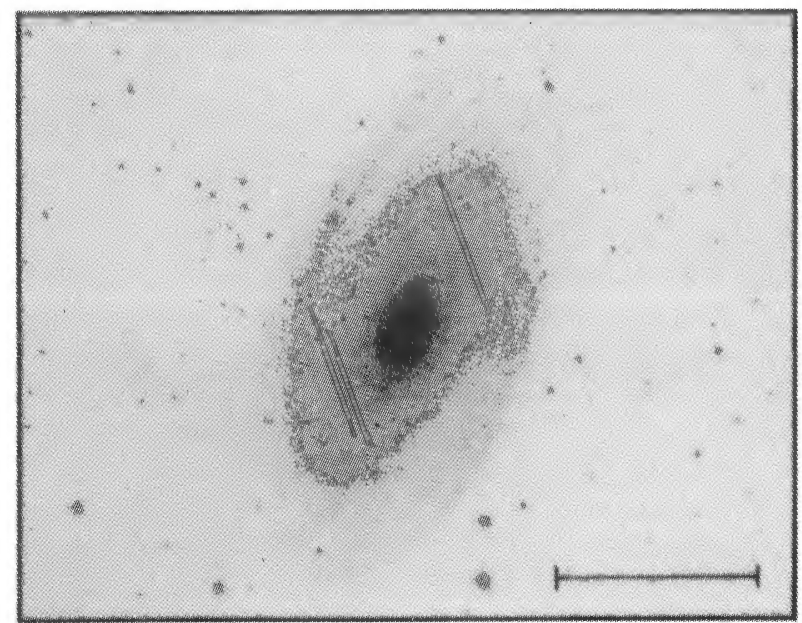

Blue Light
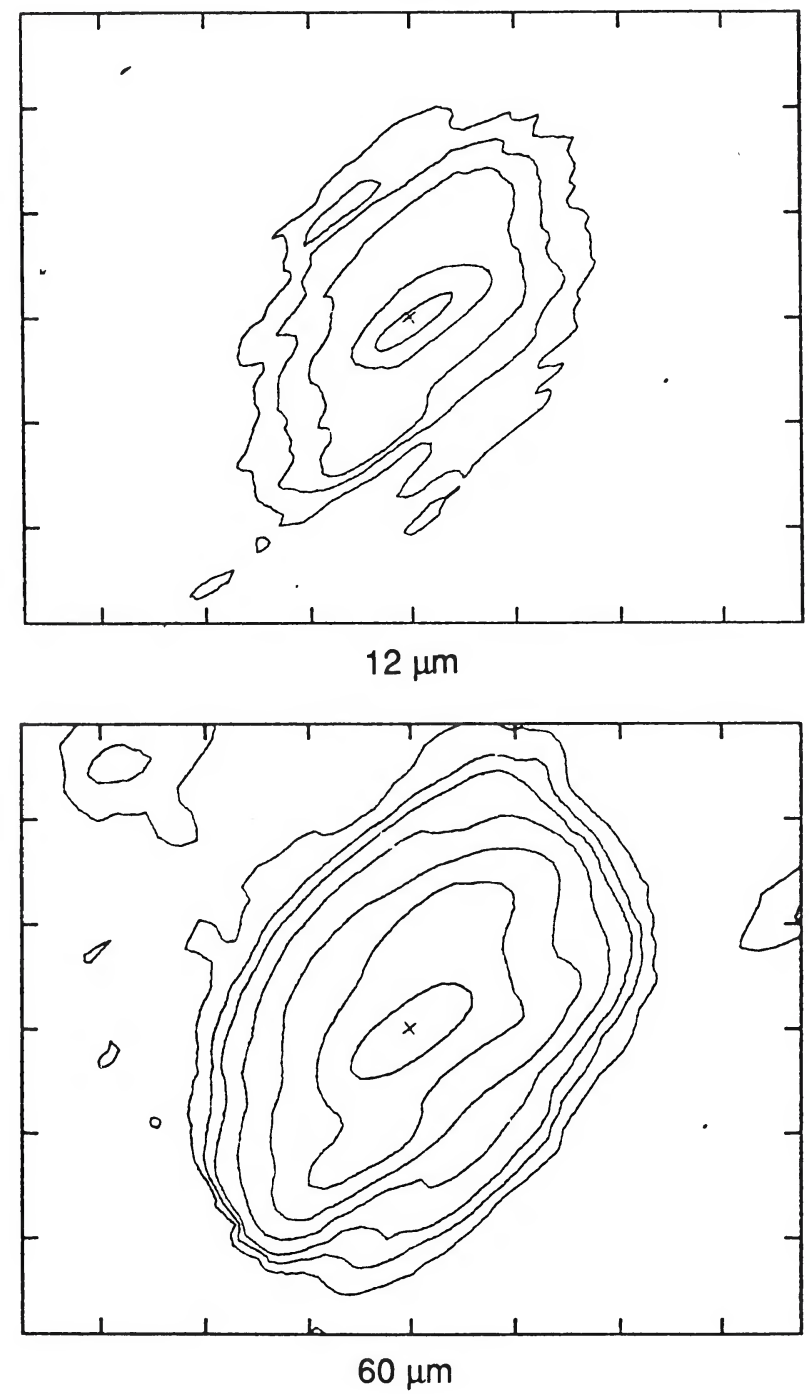

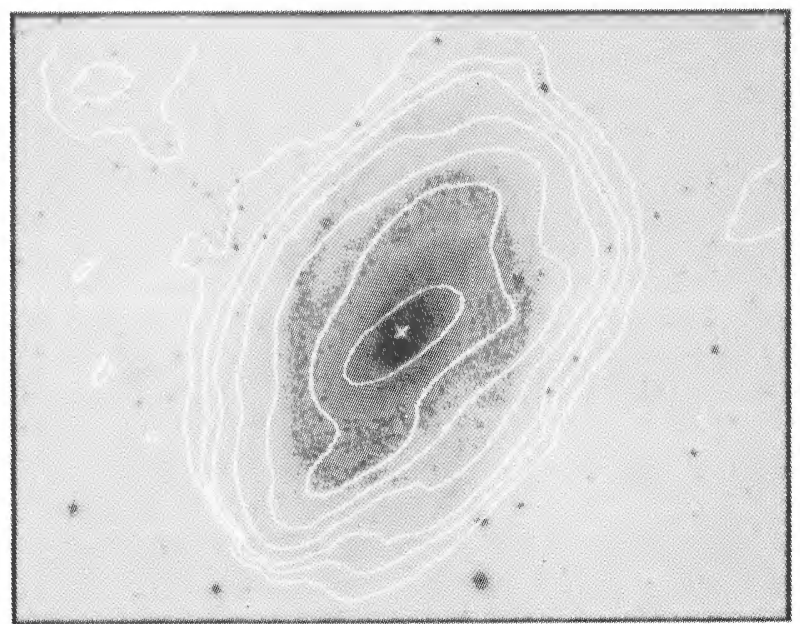

Blue and $60 \mu \mathrm{m}$
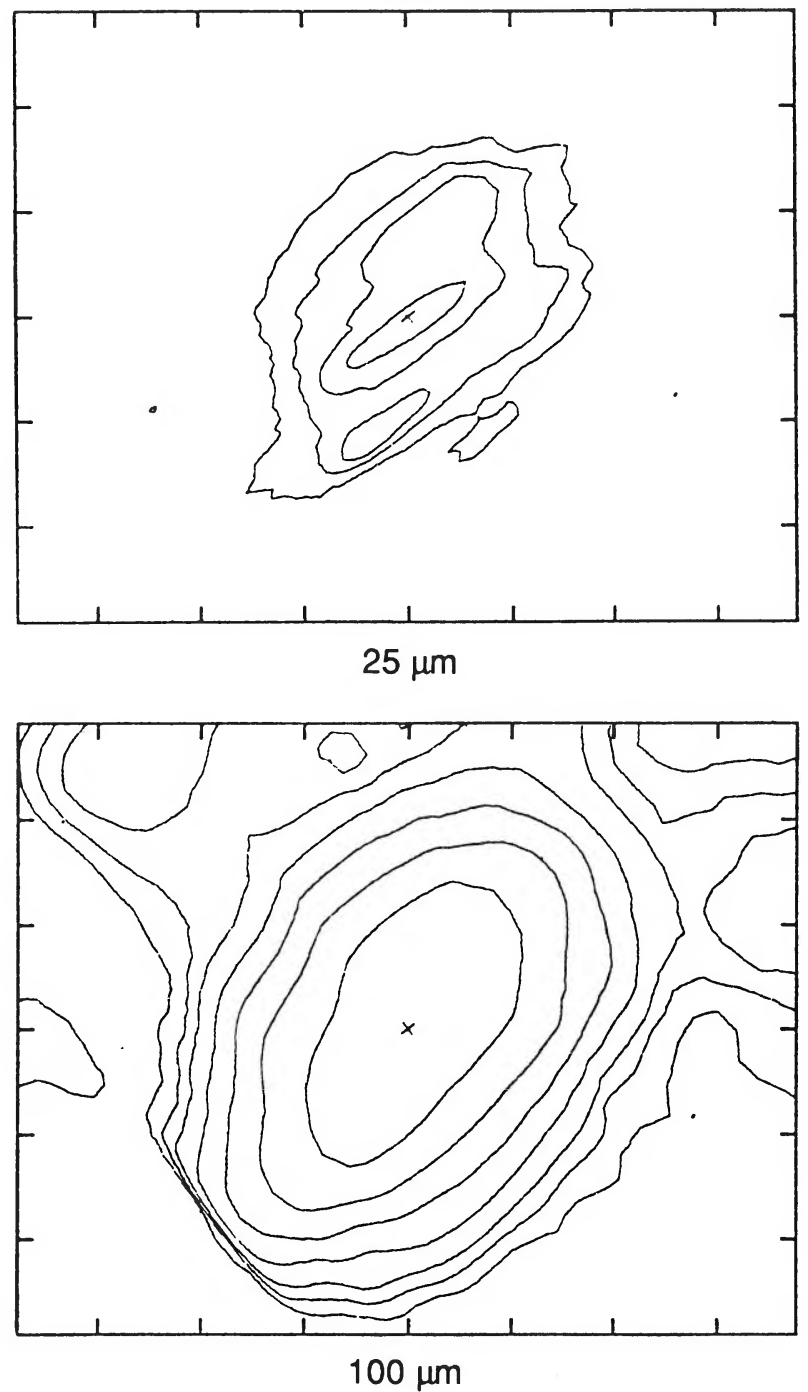

FIG. 14.- NGC 3031. (Optical photographs reproduced by permission of the California Institute of Technology. (c) 1960 National Geographic Society-Palomar Sky Survey.)

RICE et al. (see 68, 102) 


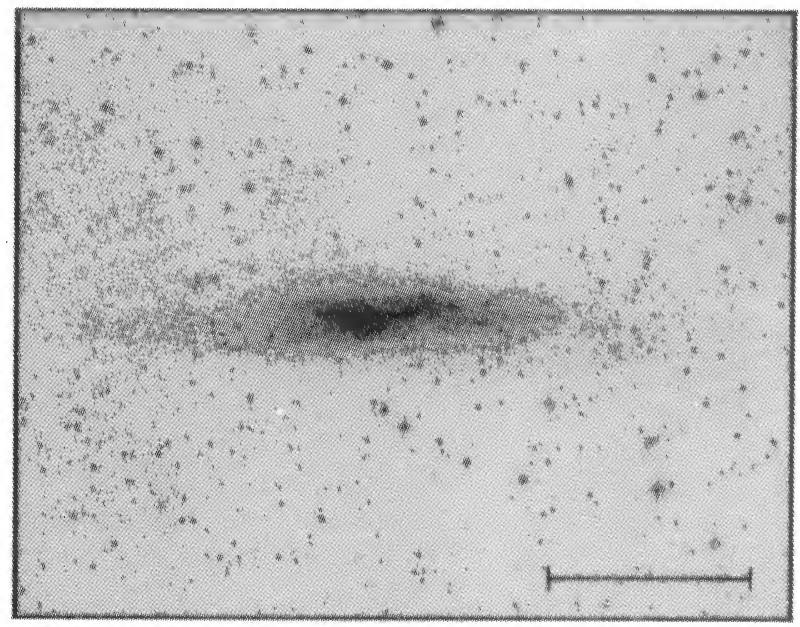

Blue Light

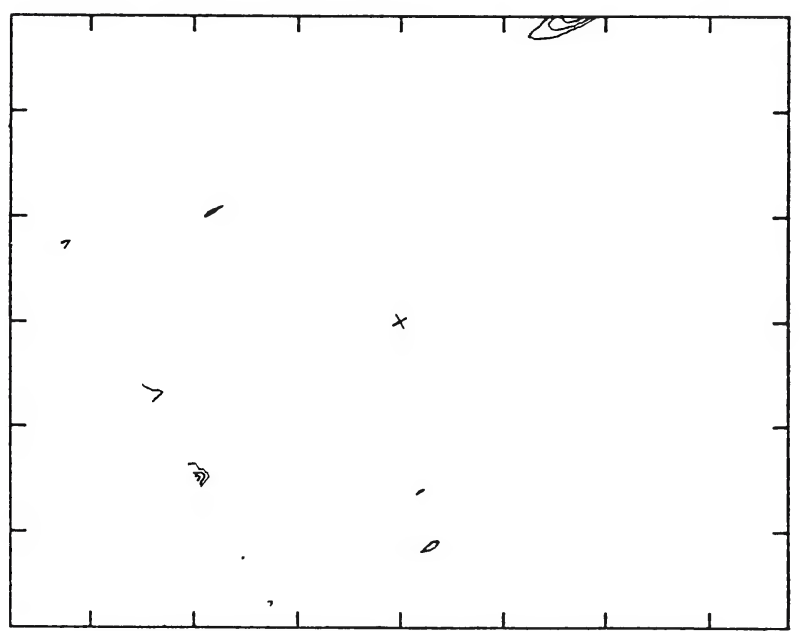

$12 \mu \mathrm{m}$

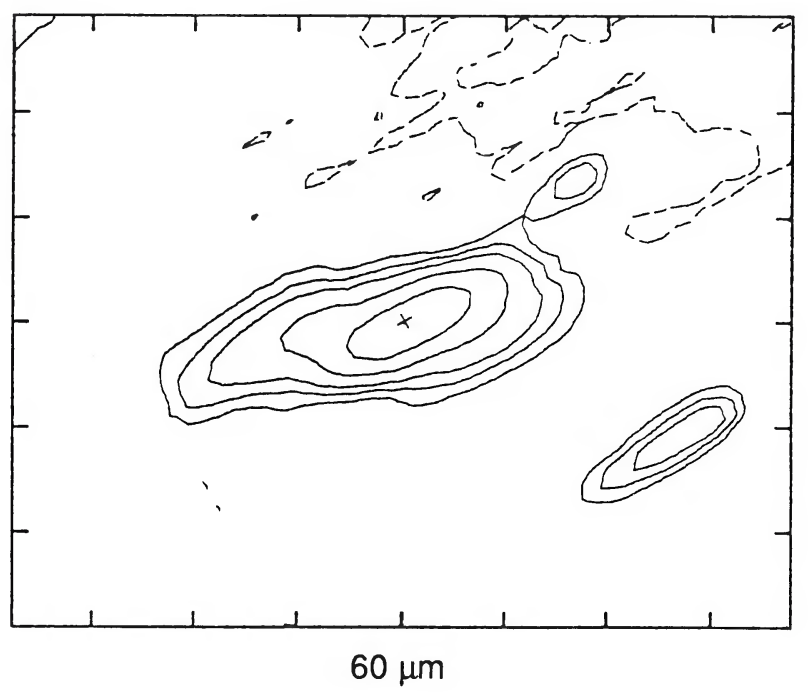

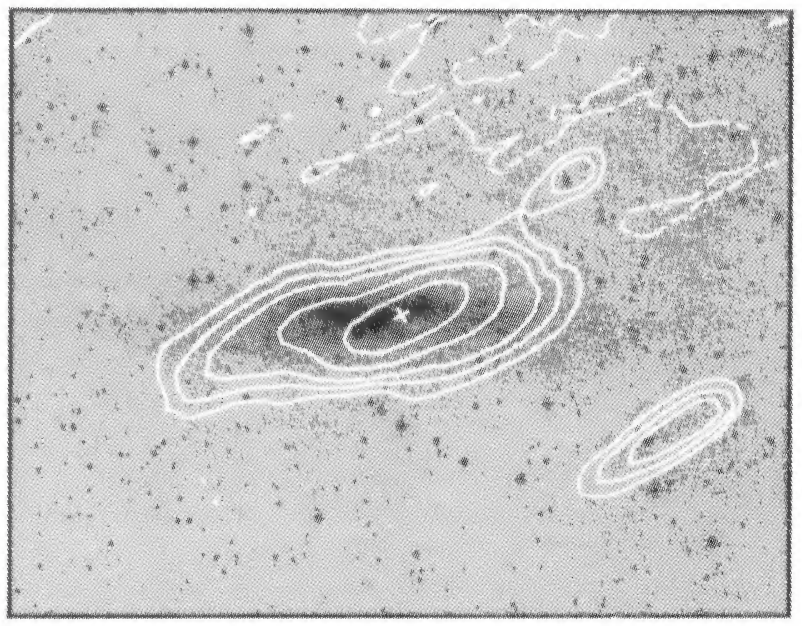

Blue and $60 \mu \mathrm{m}$

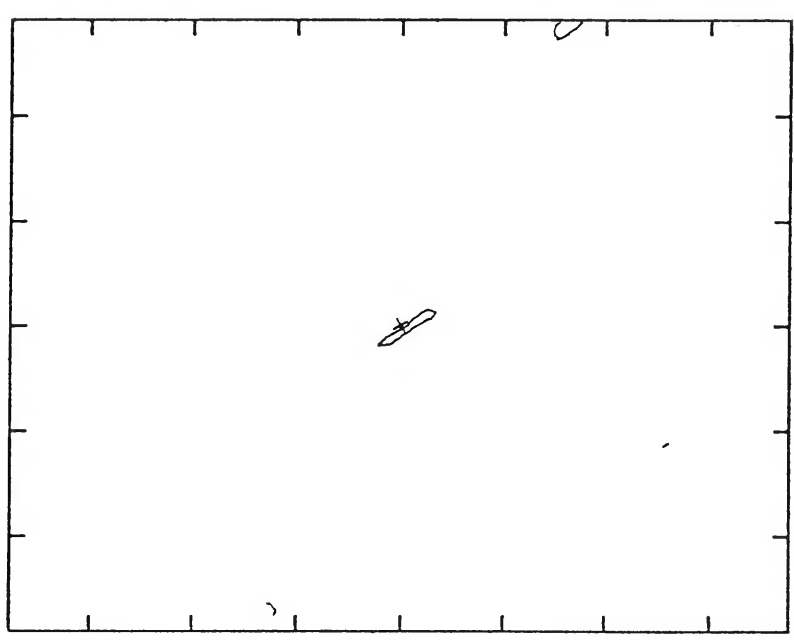

$25 \mu \mathrm{m}$

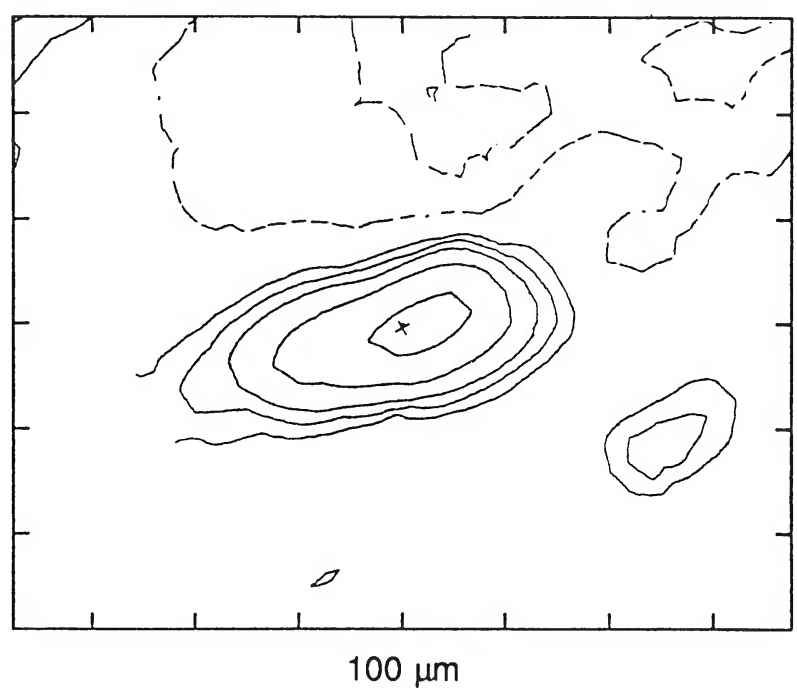

FIG. 15.-NGC 3109. (Optical photographs reproduced by permission of the European Southern Observatory.)

RICE et al. (see 68, 102) 


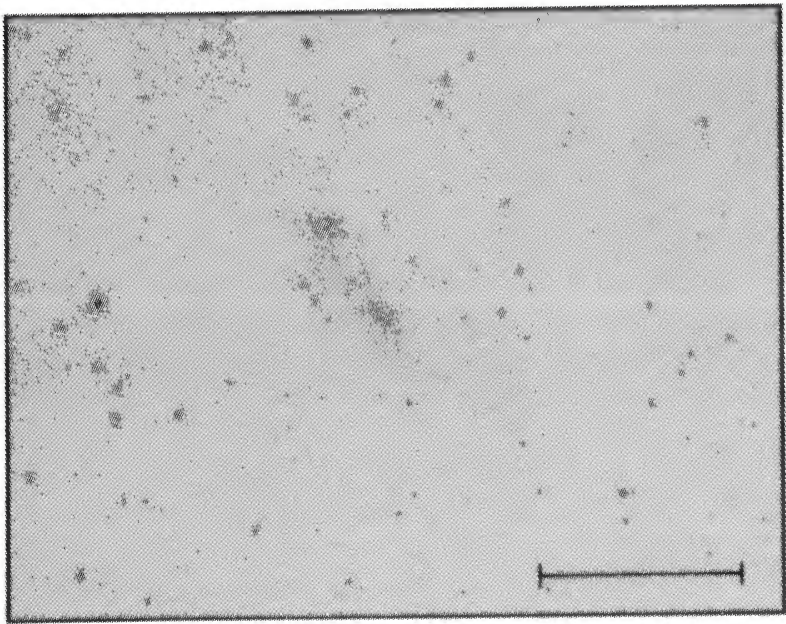

Blue Light

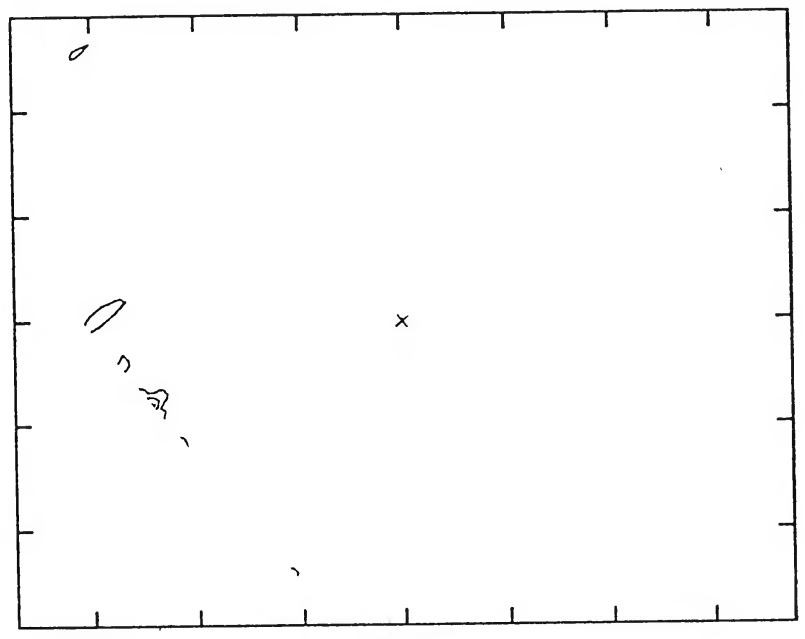

$12 \mu \mathrm{m}$

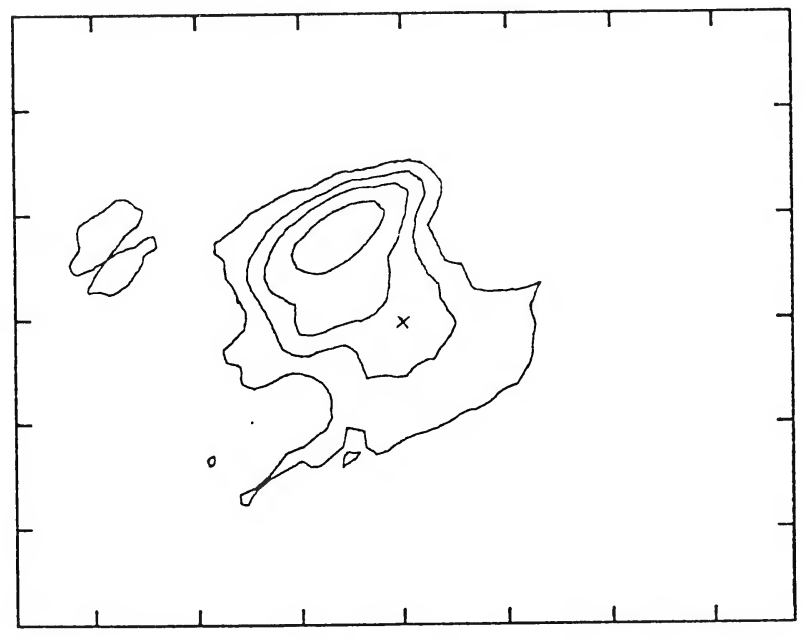

$60 \mu \mathrm{m}$

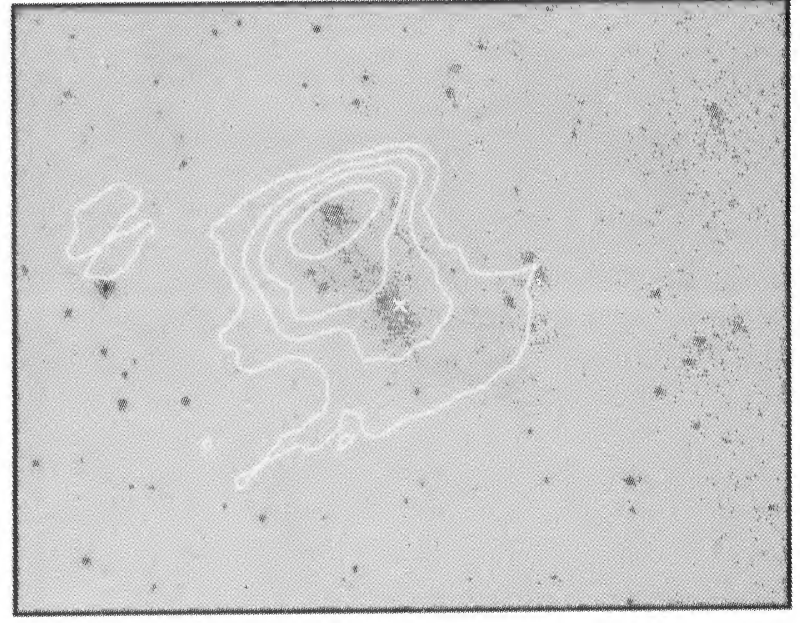

Blue and $60 \mu \mathrm{m}$
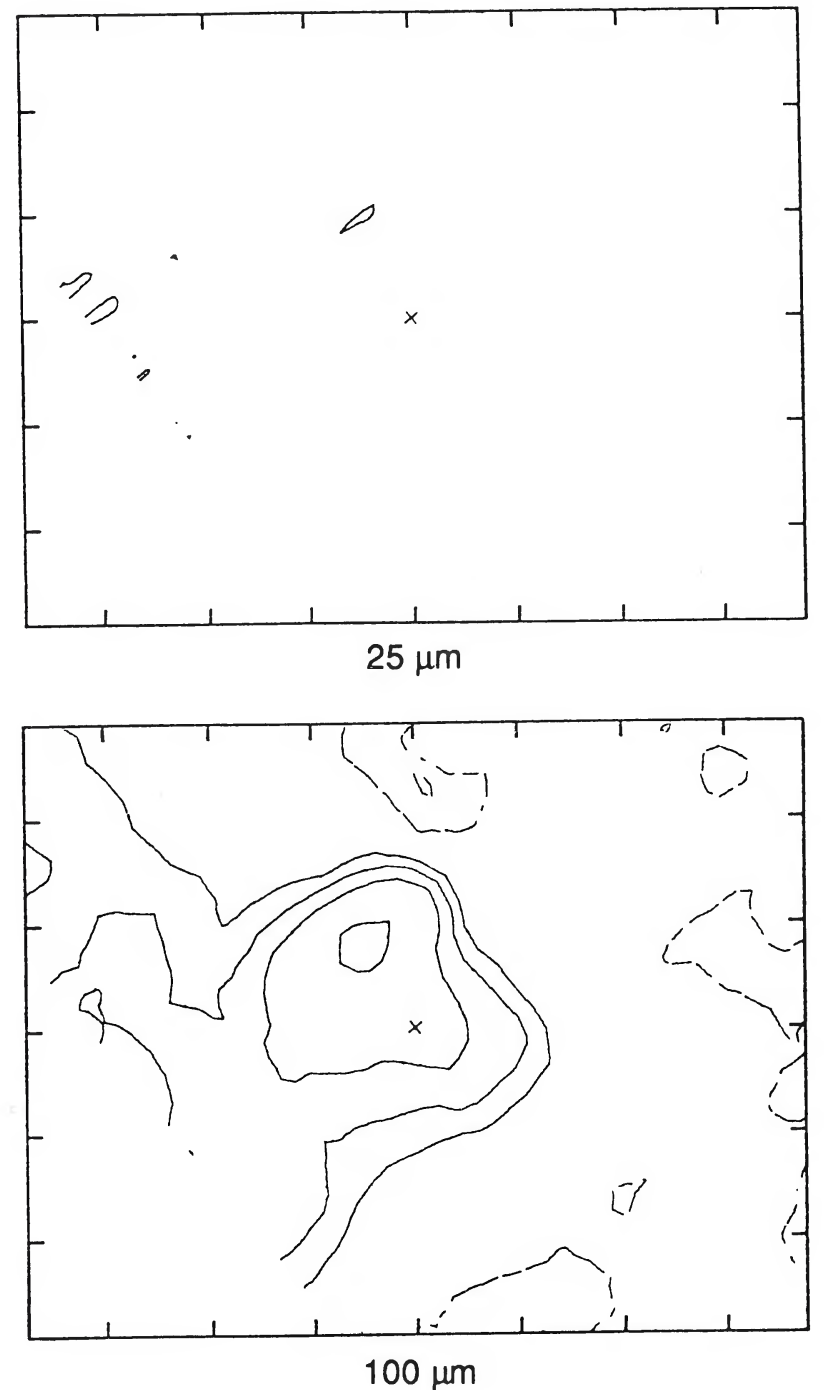

FIG. 16.-IC 2574. (Optical photographs reproduced by permission of the California Institute of Technology. (c) 1960 National Geographic Society-Palomar Sky Survey.)

Rice et al. (see 68, 102) 


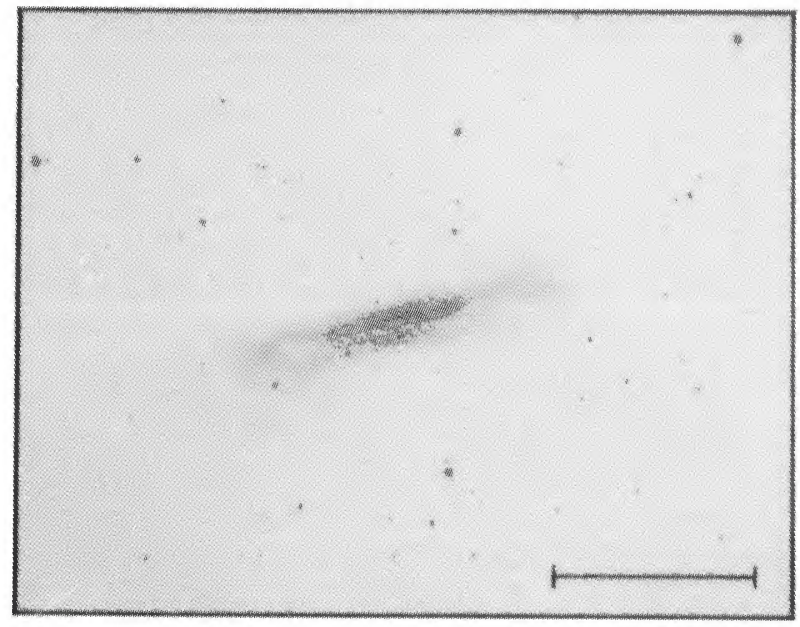

Blue Light

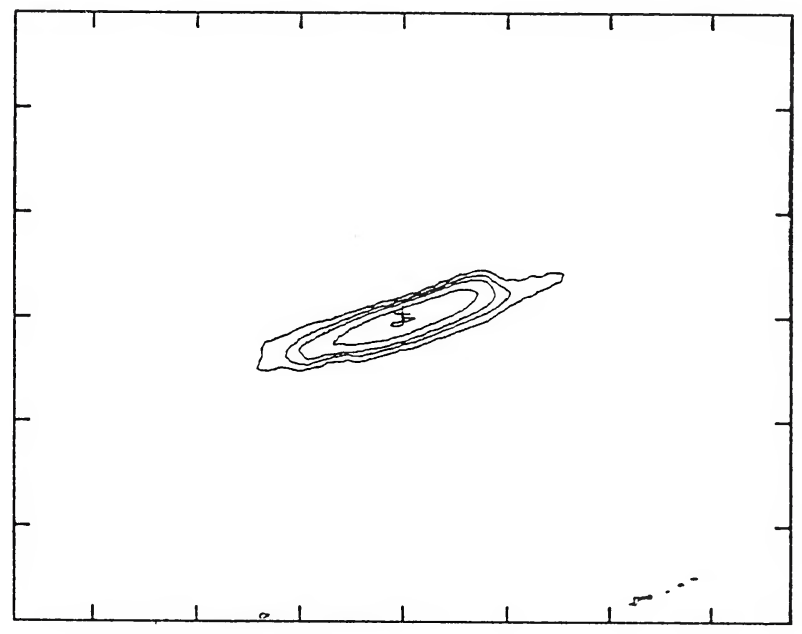

$12 \mu \mathrm{m}$

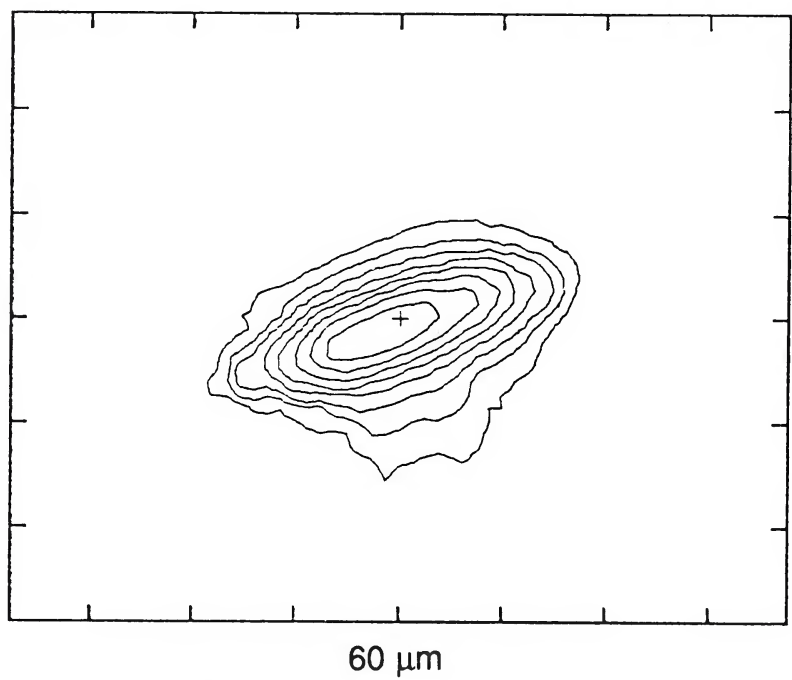

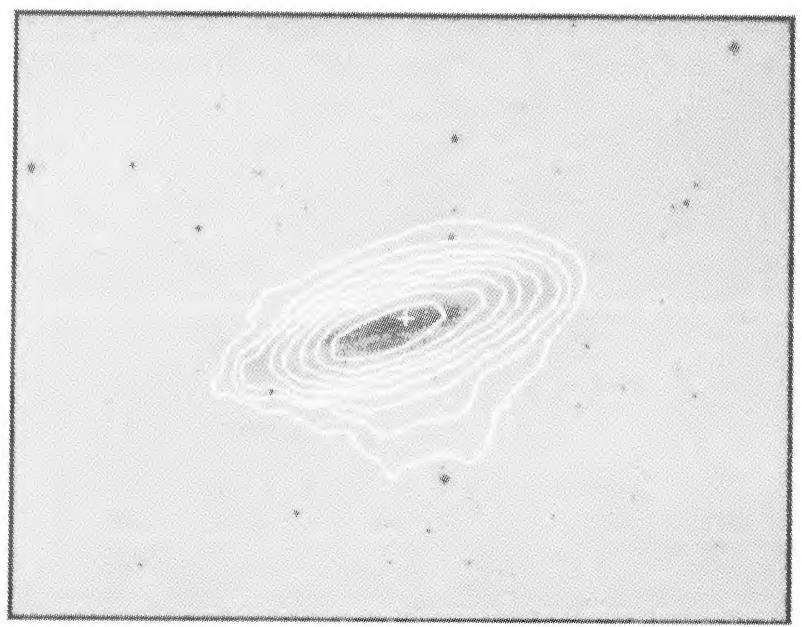

Blue and $60 \mu \mathrm{m}$

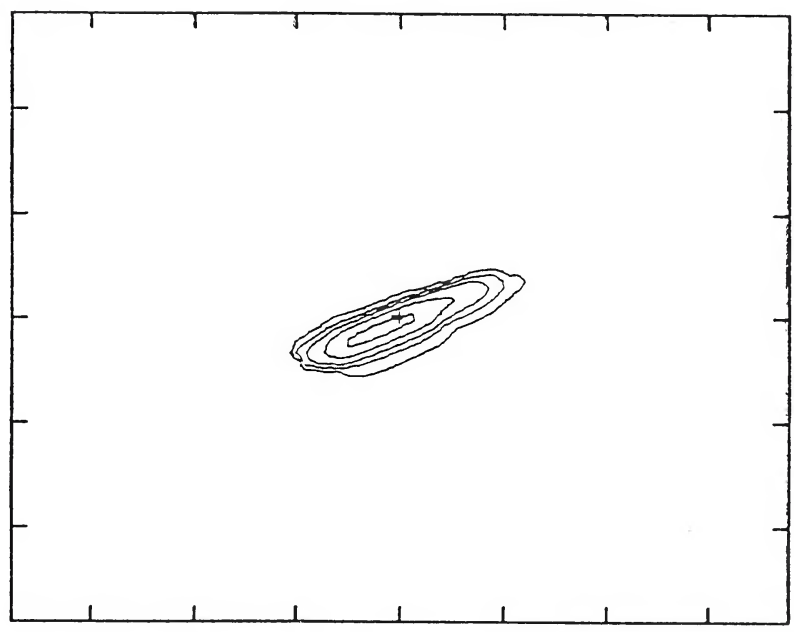

$25 \mu \mathrm{m}$

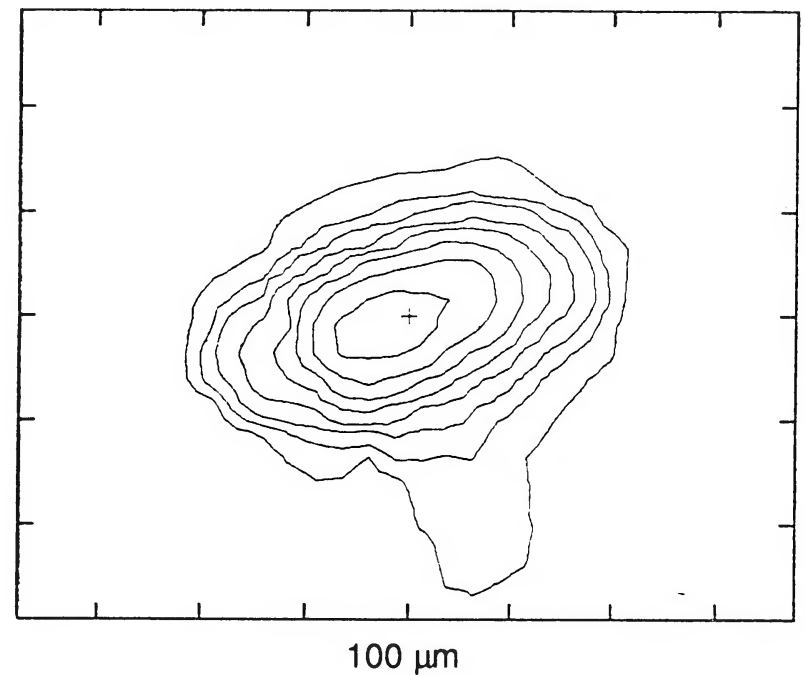

FIg. 17.-NGC 3628. (Optical photographs reproduced by permission of the California Institute of Technology. (C) 1960 National Geographic Society-Palomar Sky Survey.)

RicE et al. (see 68, 102) 


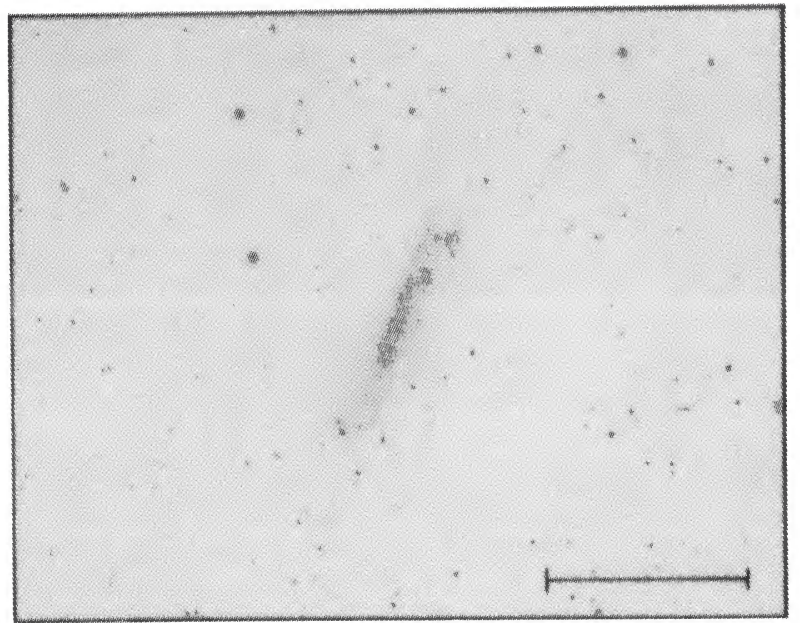

Blue Light

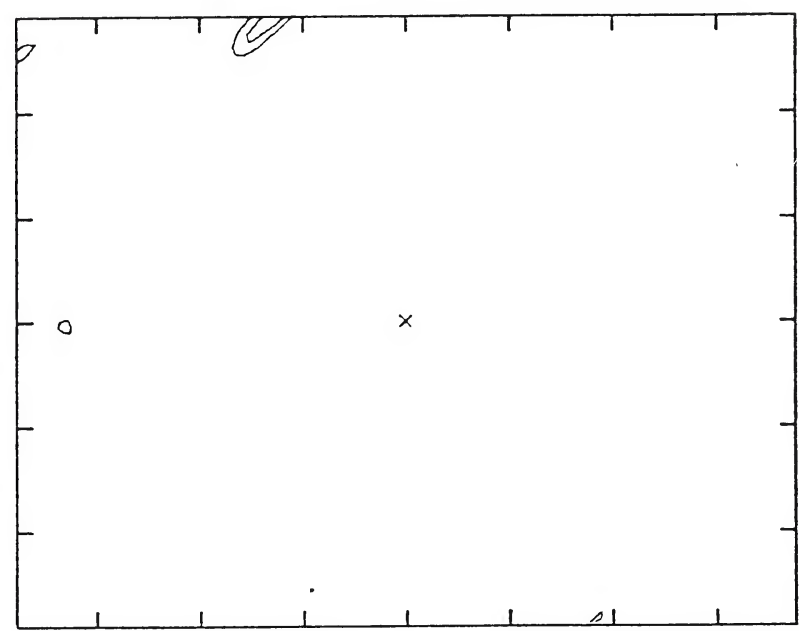

$12 \mu \mathrm{m}$

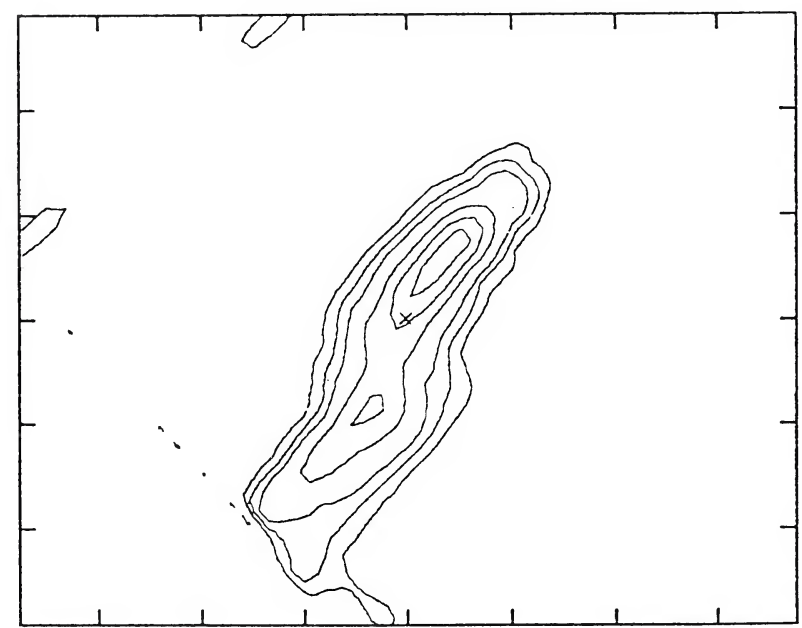

$60 \mu \mathrm{m}$

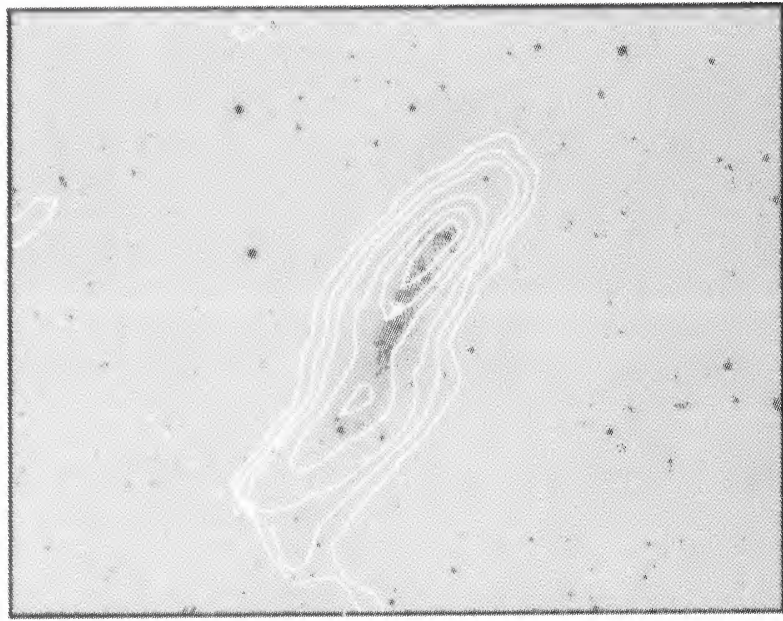

Blue and $60 \mu \mathrm{m}$

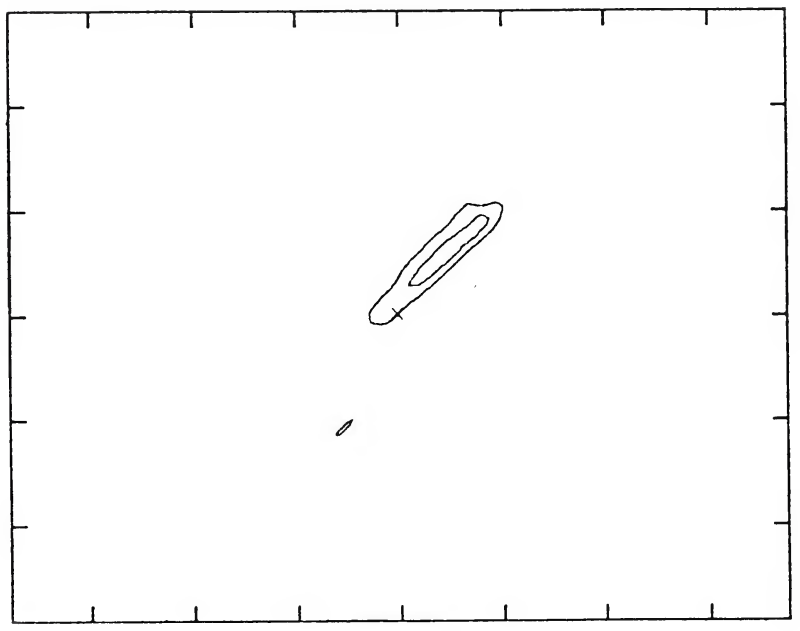

$25 \mu \mathrm{m}$

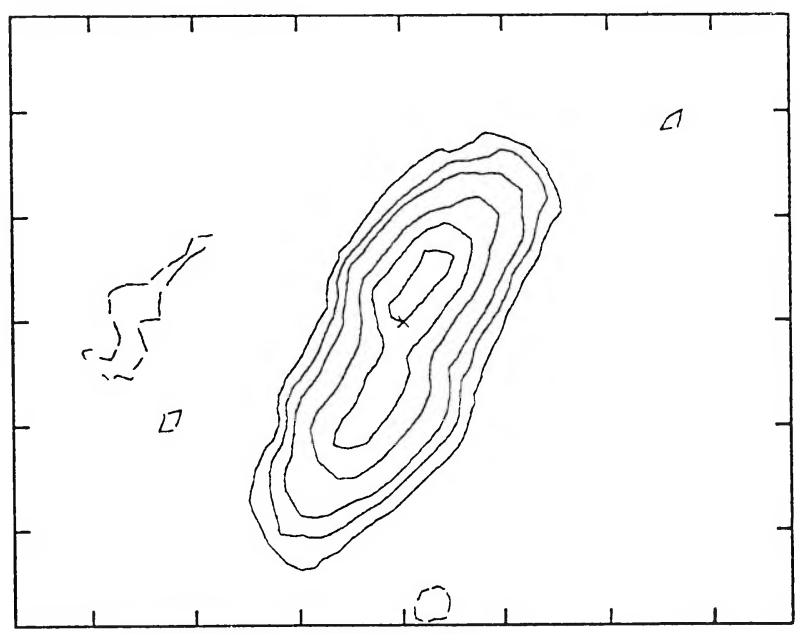

$100 \mu \mathrm{m}$

FIG. 18. - NGC 4236. The map contour levels are at (dashed) 3,6, and $9 \sigma$ below the map mean background level and at (solid) 3,6 , and $10 \sigma$ with brighter levels at increments of $10 \sigma$. (Optical photographs reproduced by permission of the California Institute of Technology. (C) 1960 National Geographic Society-Palomar Sky Survey.)

RICE et al. (see 68, 102) 


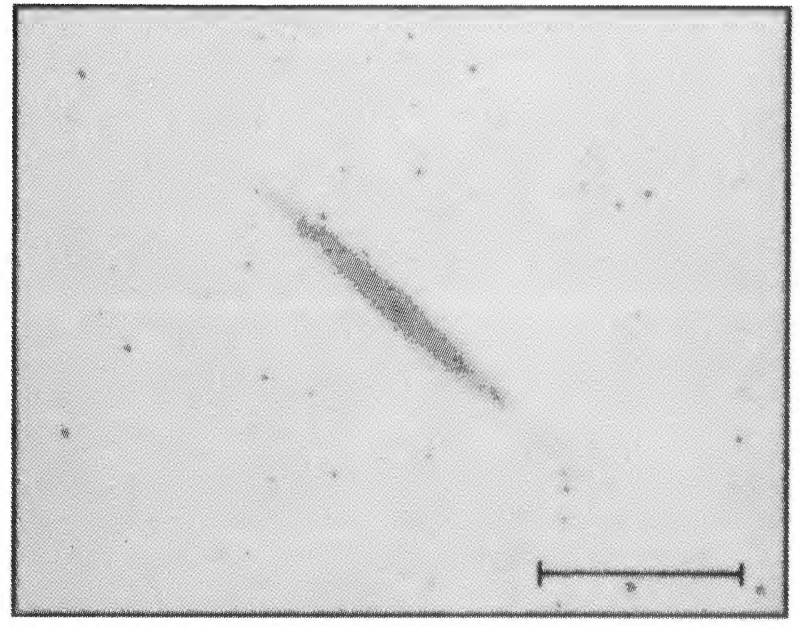

Blue Light

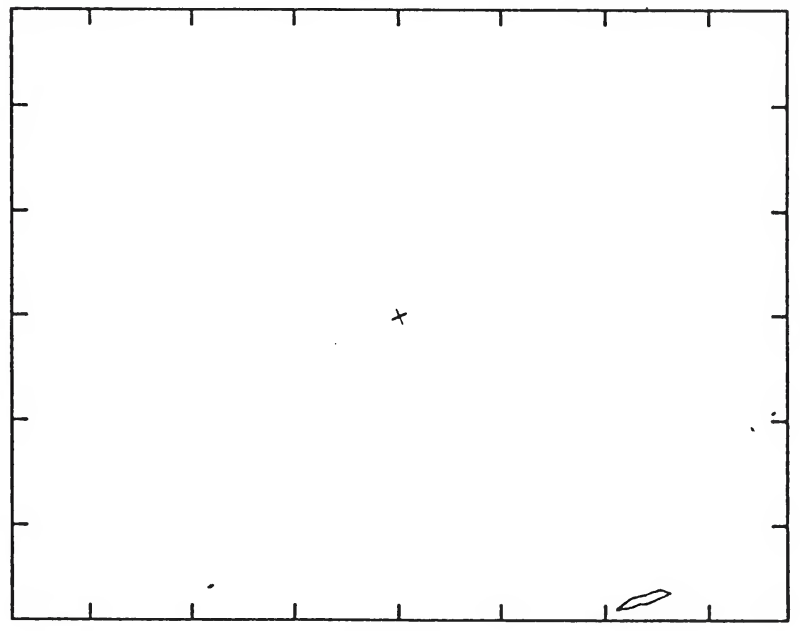

$12 \mu \mathrm{m}$

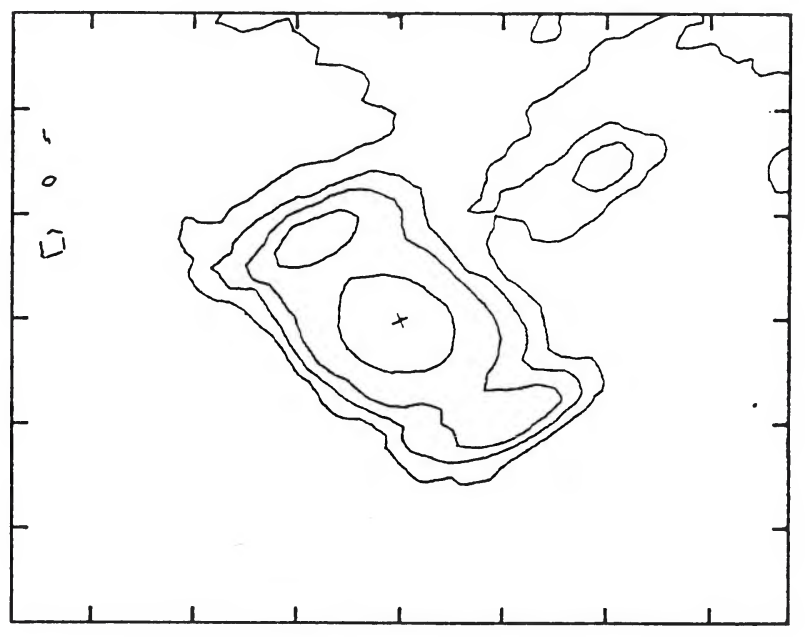

$60 \mu \mathrm{m}$

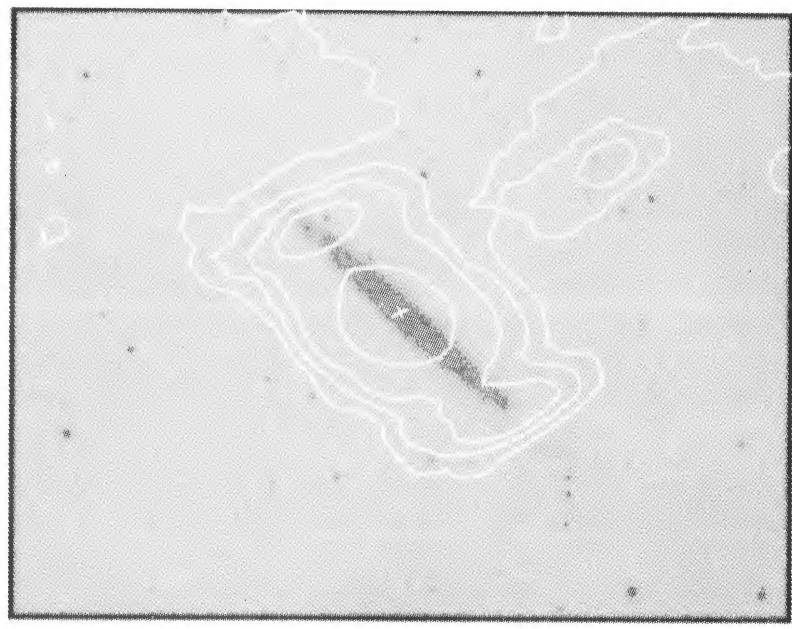

Blue and $60 \mu \mathrm{m}$

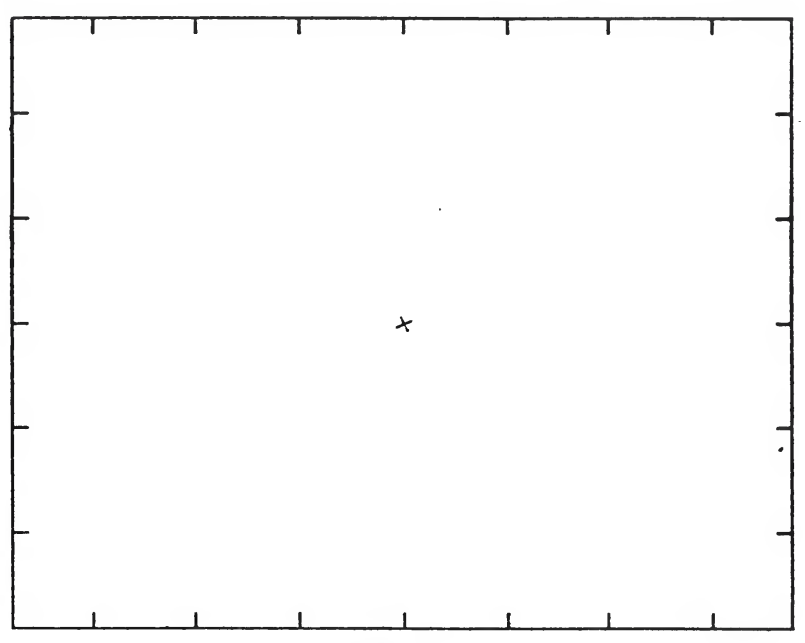

$25 \mu \mathrm{m}$

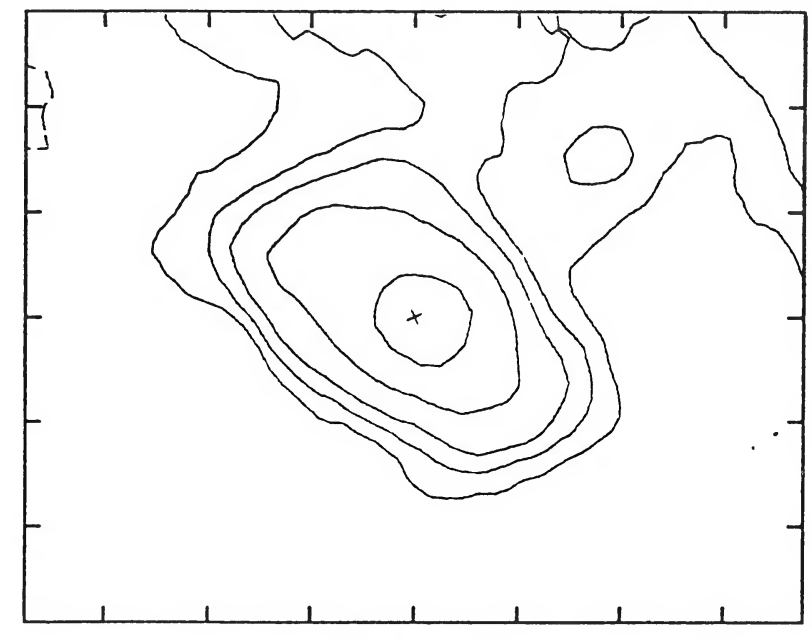

$100 \mu \mathrm{m}$

FIG. 19.-NGC 4244. (Optical photographs reproduced by permission of the California Institute of Technology. (c) 1960 National Geographic Society-Palomar Sky Survey.)

RICE et al. (see 68, 102) 


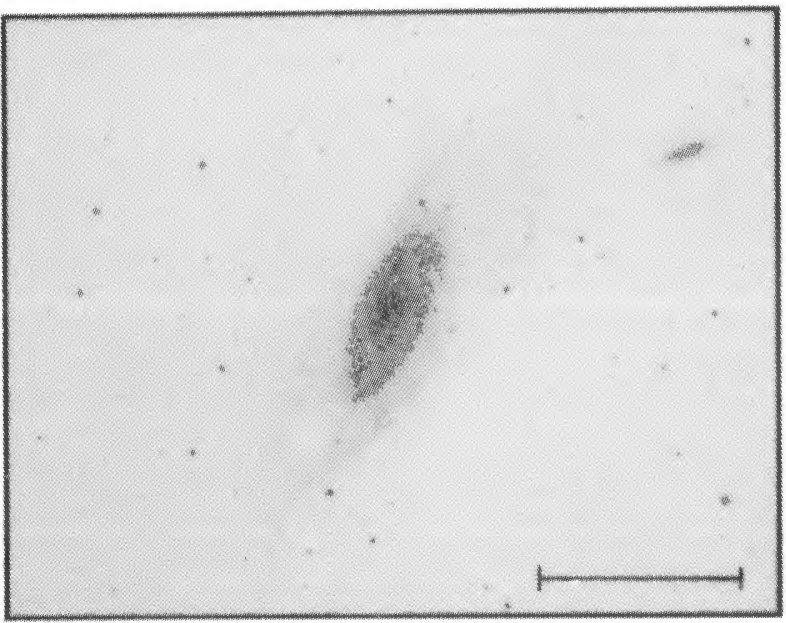

Blue Light
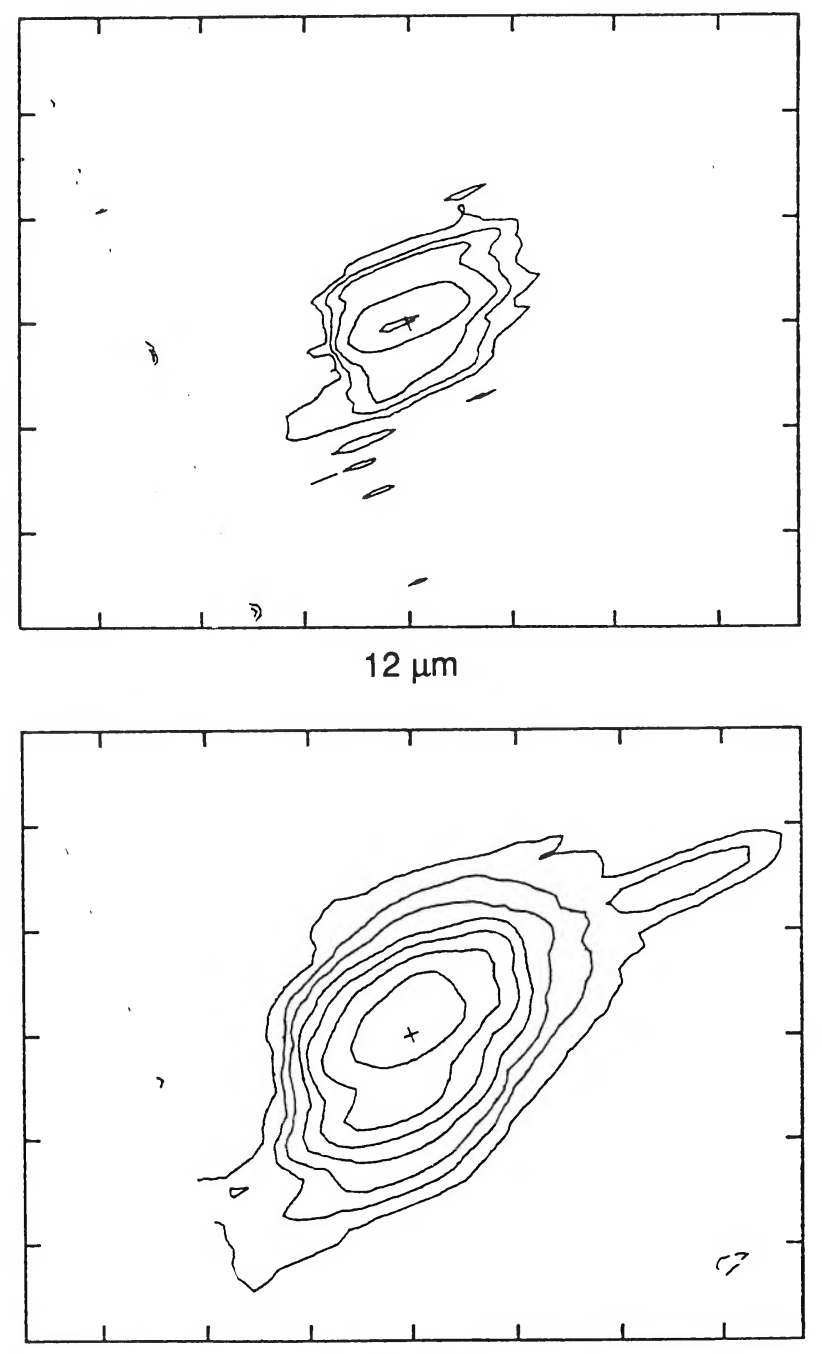

$60 \mu \mathrm{m}$

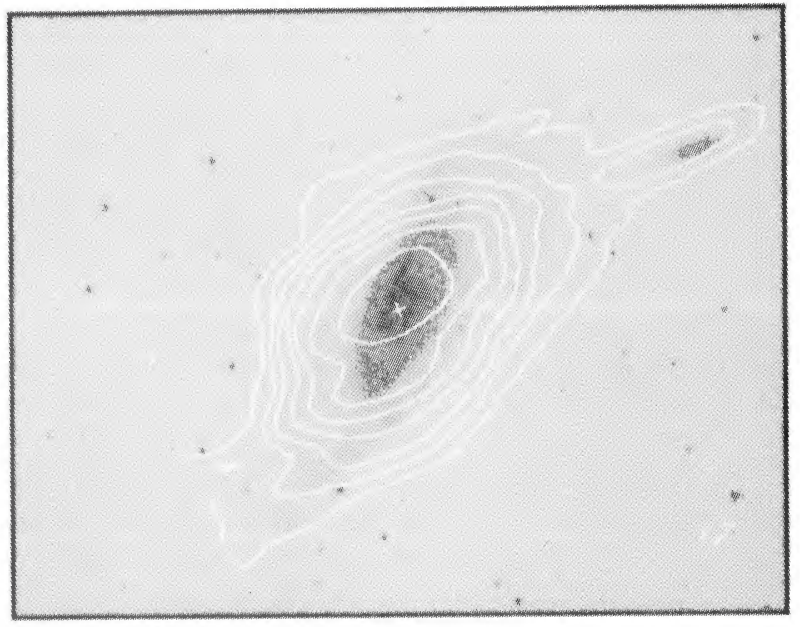

Blue and $60 \mu \mathrm{m}$

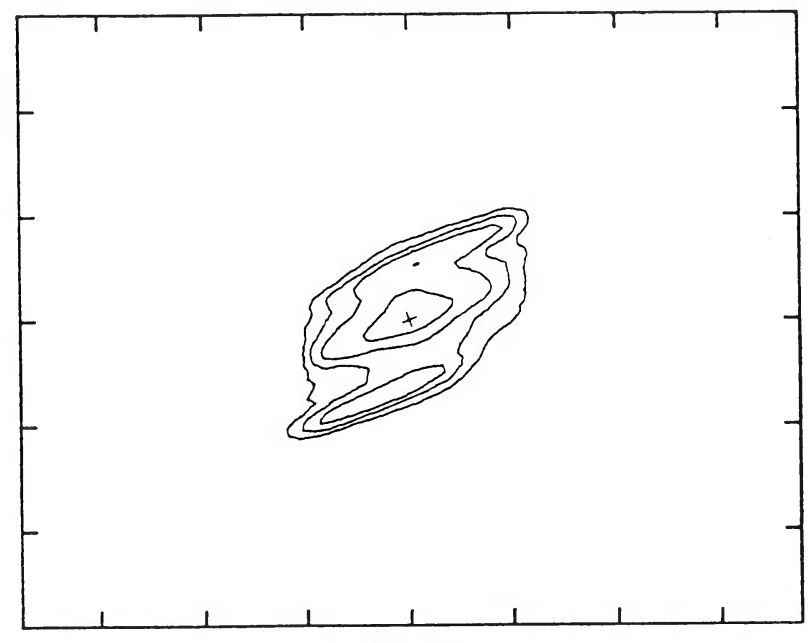

$25 \mu \mathrm{m}$

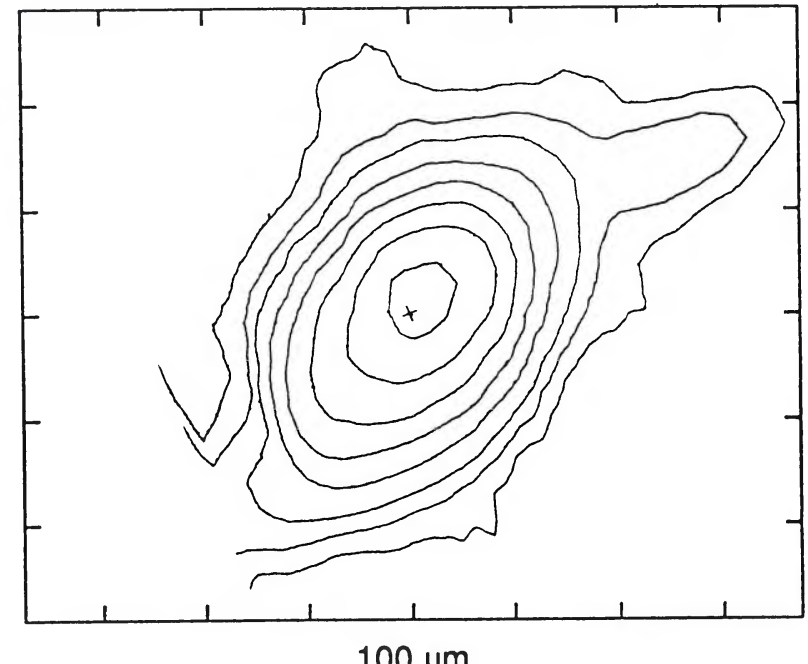

$100 \mu \mathrm{m}$

FIG. 20.-NGC 4258. (Optical photographs reproduced by permission of the California Institute of Technology. (1) 1960 National Geographic Society-Palomar Sky Survey.)

Rice et al. (see 68, 102) 


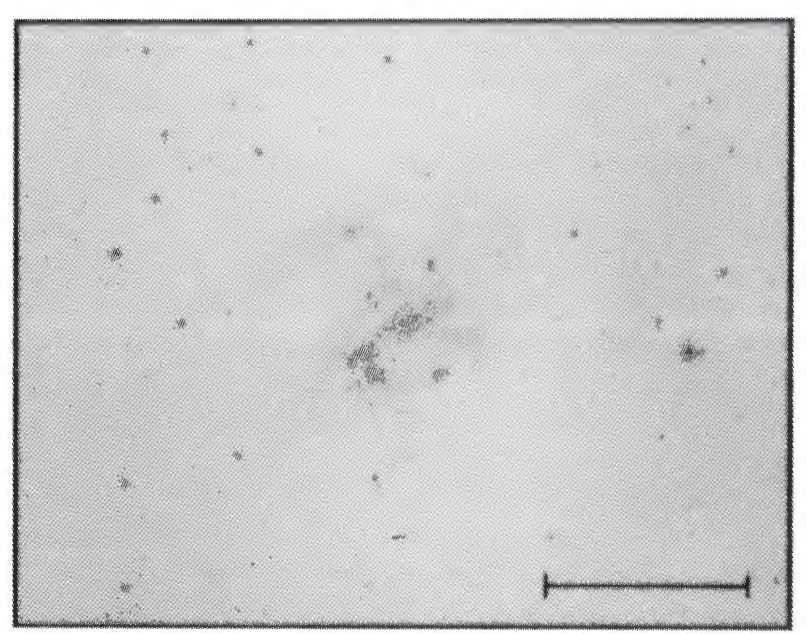

Blue Light

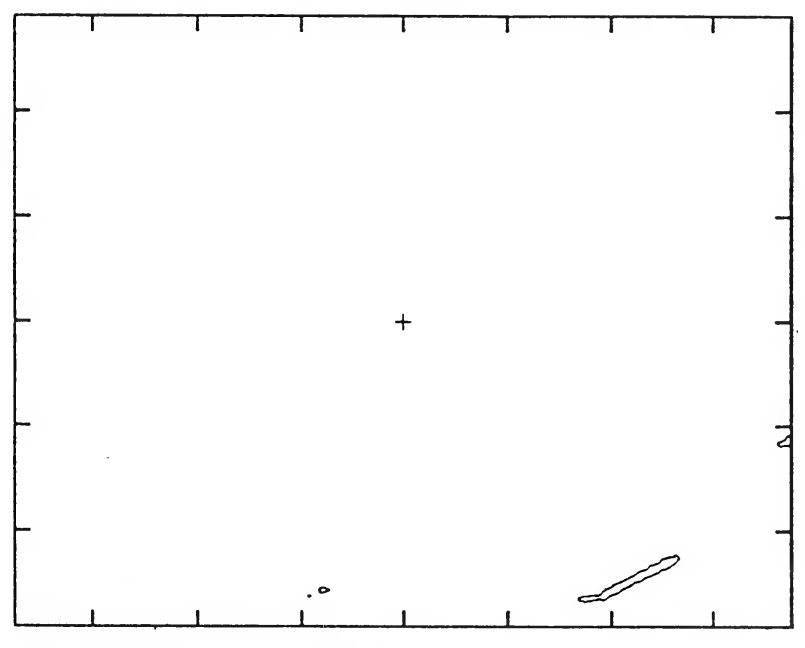

$12 \mu \mathrm{m}$

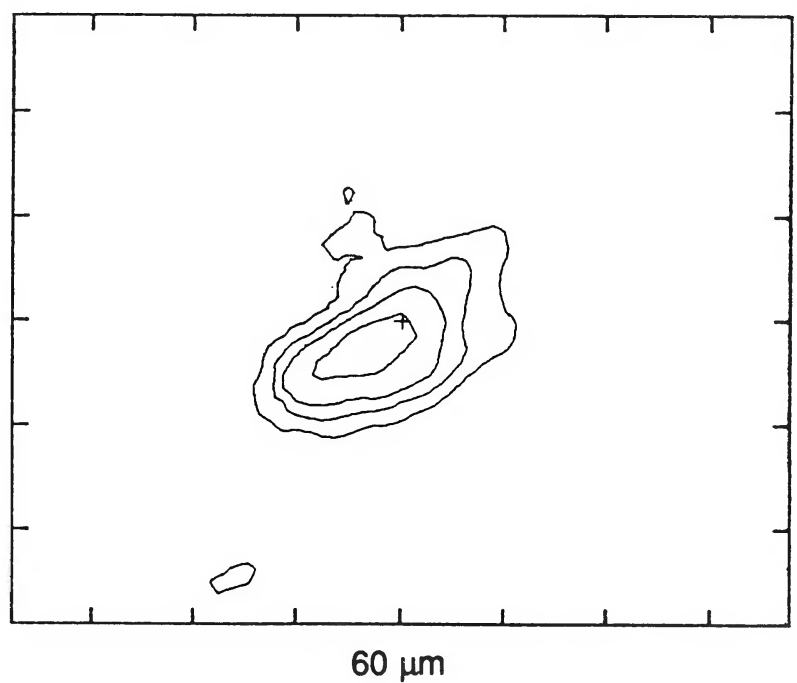

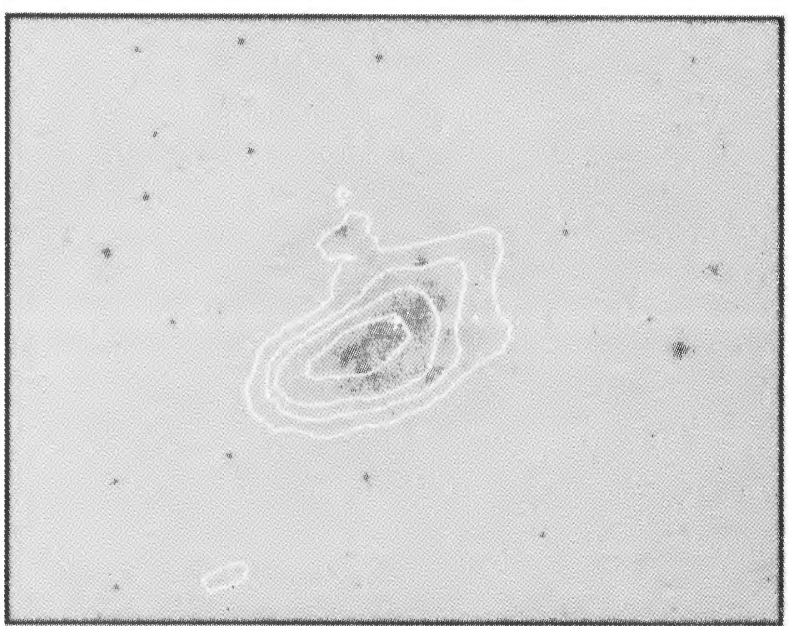

Blue and $60 \mu \mathrm{m}$
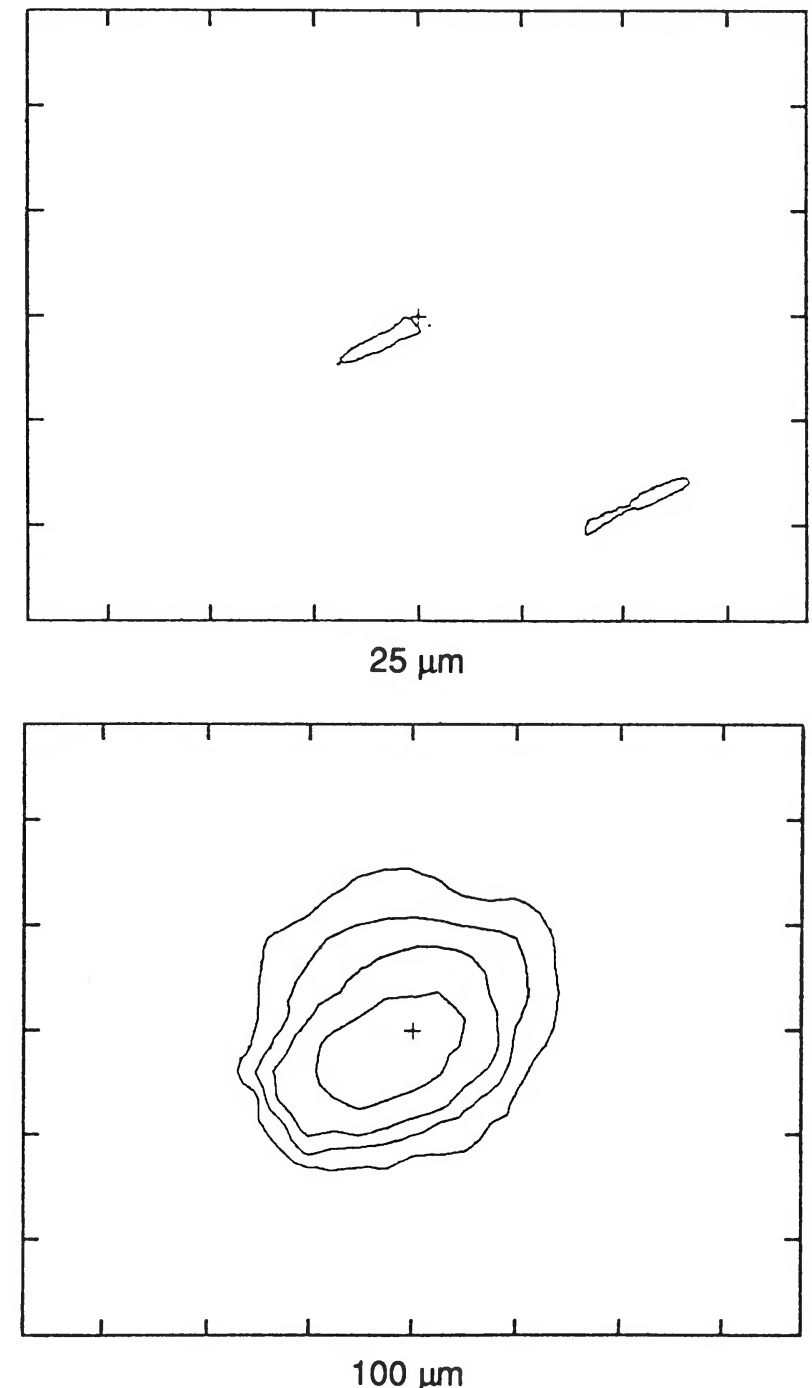

FIG. 21.-NGC 4395. (Optical photographs reproduced by permission of the California Institute of Technology. (C) 1960 National Geographic Society-Palomar Sky Survey.)

RICE et al. (see 68, 102) 


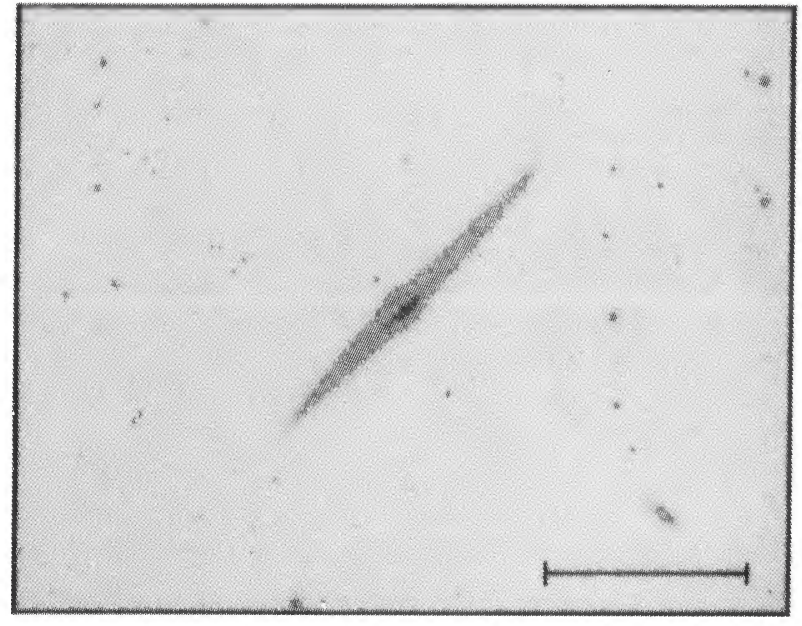

Blue Light
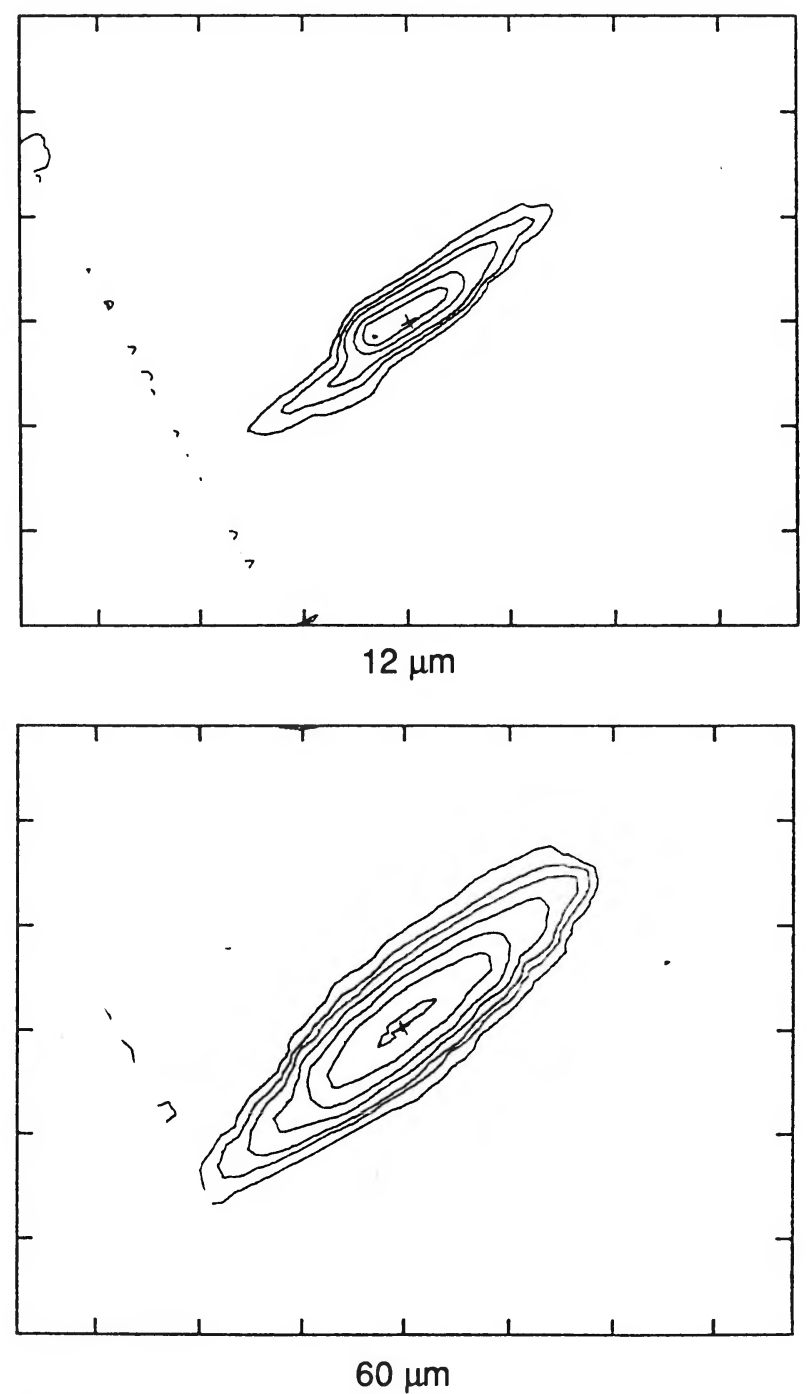

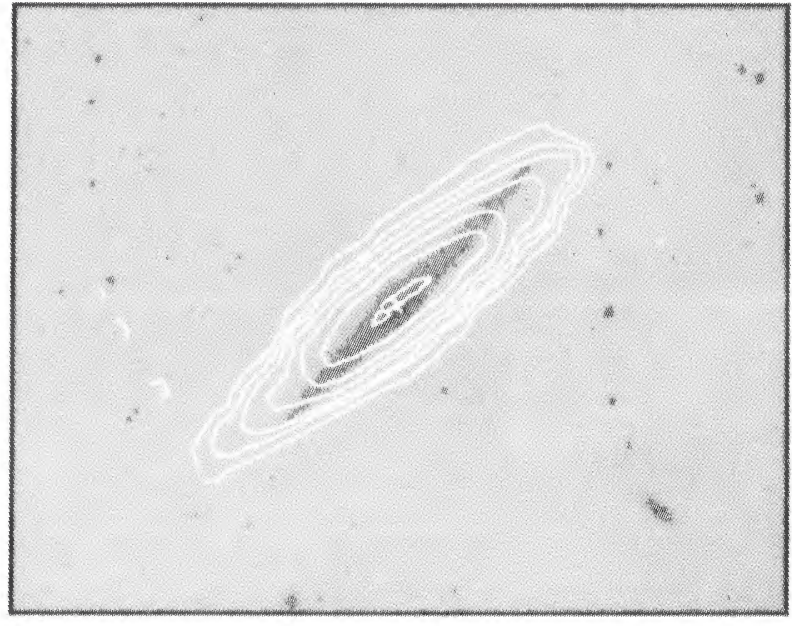

Blue and $60 \mu \mathrm{m}$
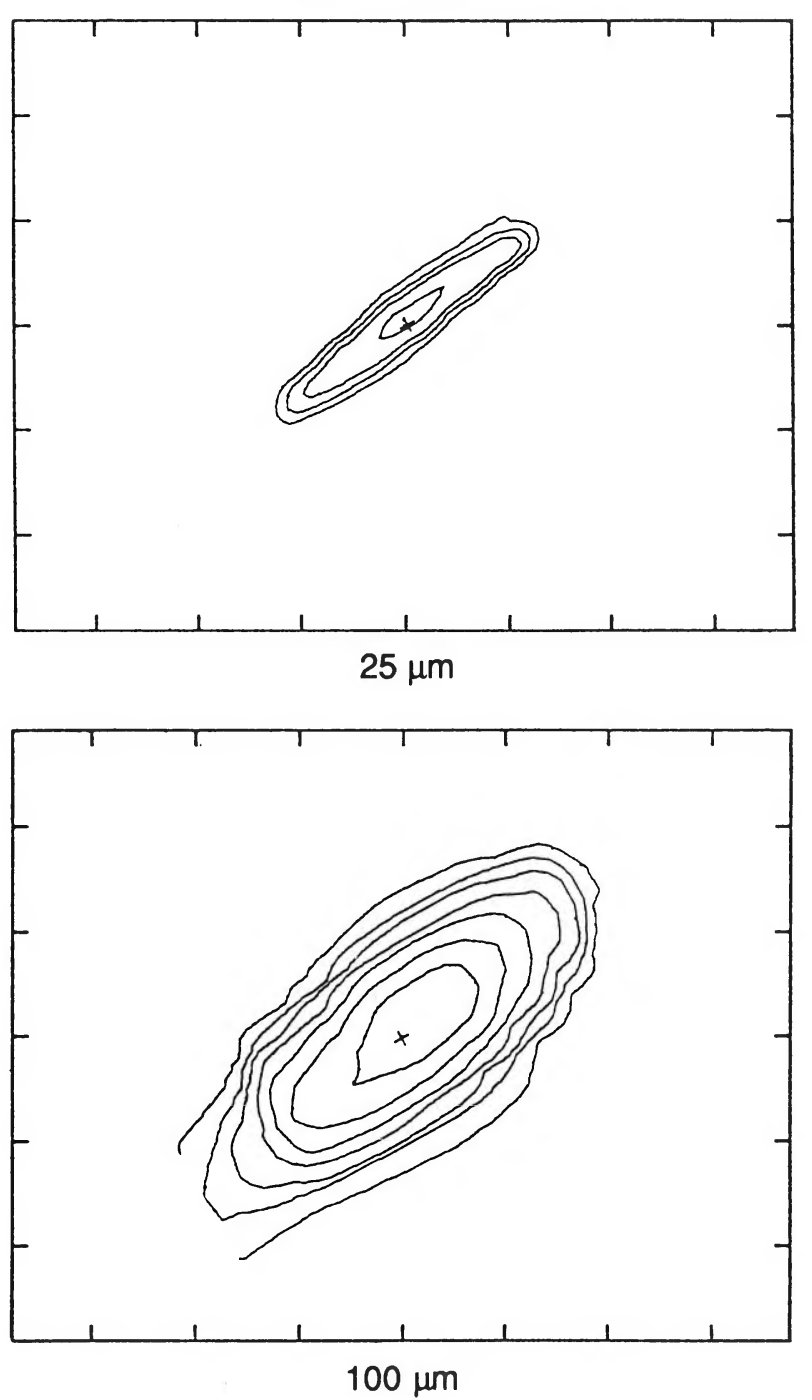

FIG. 22.-NGC 4565. For the 12 and $25 \mu \mathrm{m}$ maps, the contour levels are at (dashed) 3, 6, and $9 \sigma$ below the map mean background level and at (solid) 3, 6, and $10 \sigma$ with brighter levels at increments of $10 \sigma$. (Optical photographs reproduced by permission of the California Institute of Technology. (c) 1960 National Geographic Society-Palomar Sky Survey.)

RICE et al. (see 68, 102) 


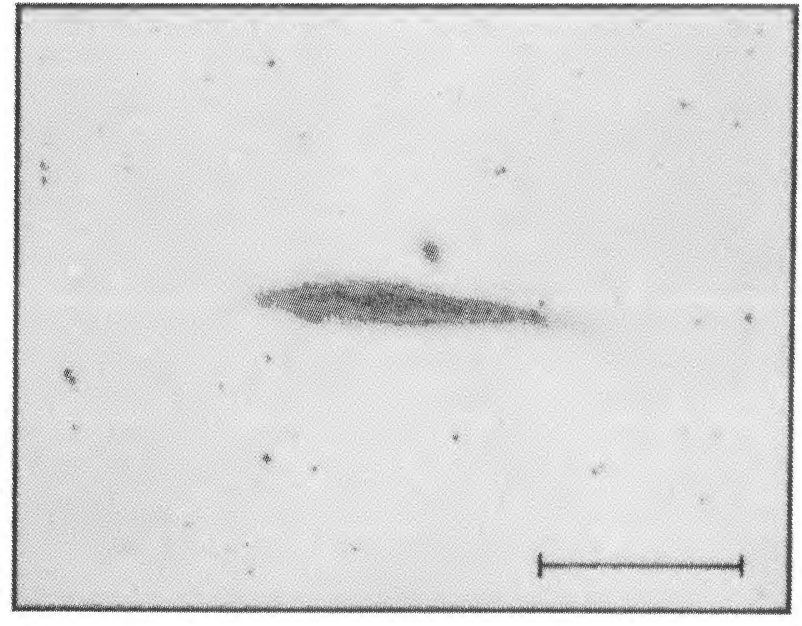

Blue Light
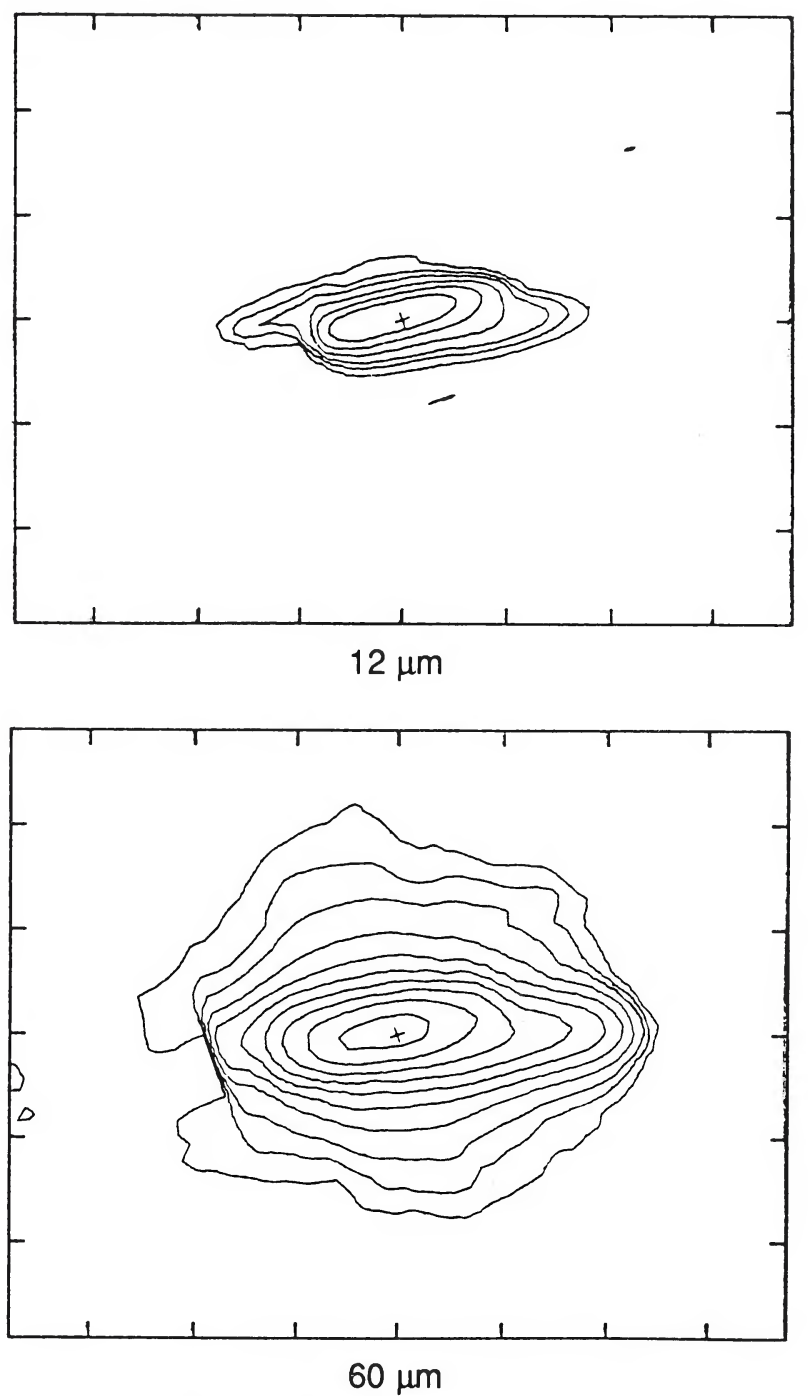

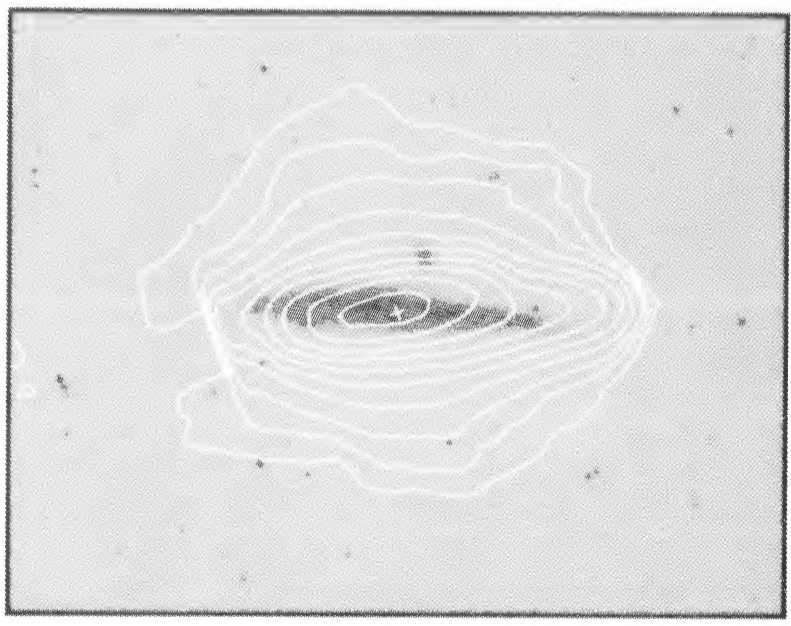

Blue and $60 \mu \mathrm{m}$
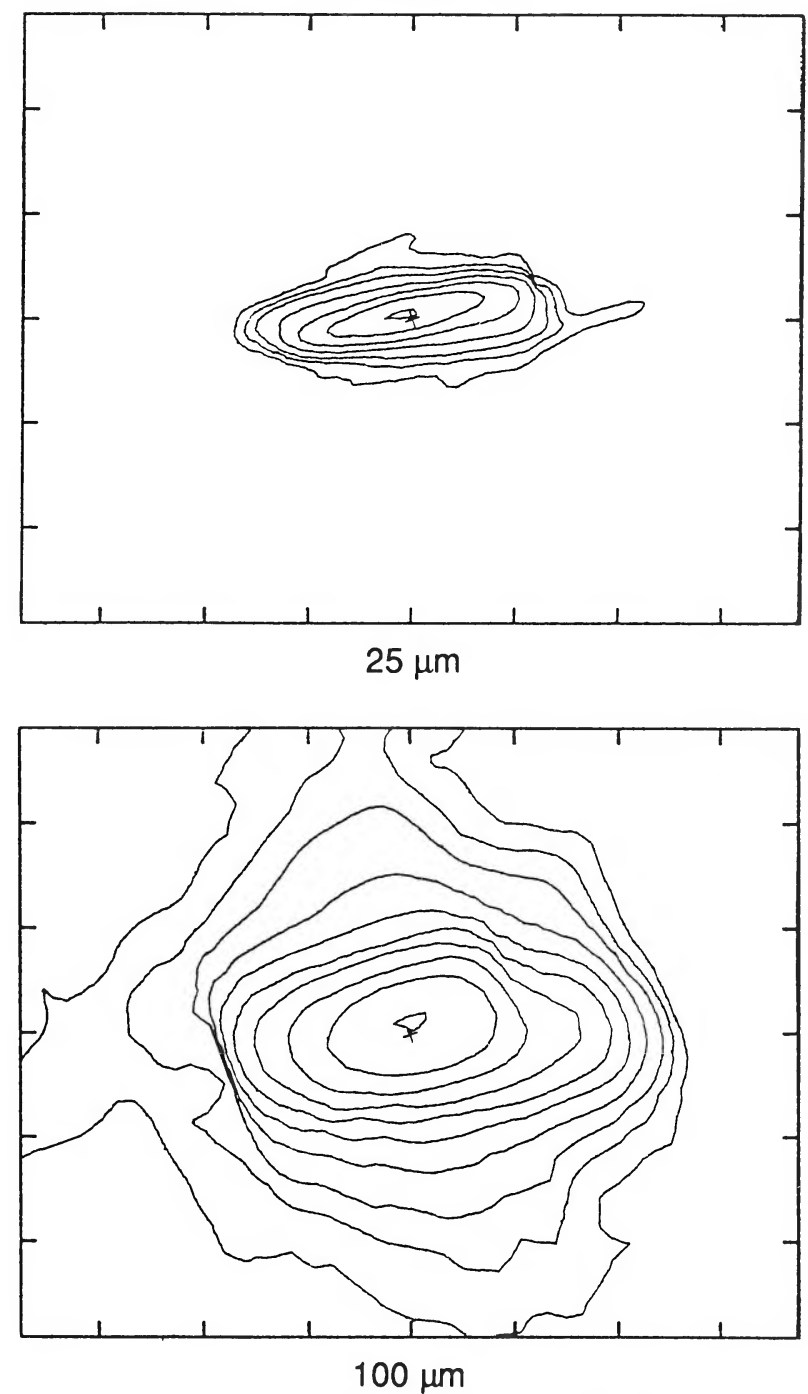

FIG. 23.-NGC 4631. (Optical photographs reproduced by permission of the California Institute of Technology. (c) 1960 National Geographic Society-Palomar Sky Survey.)

RicE et al. (see 68, 102) 


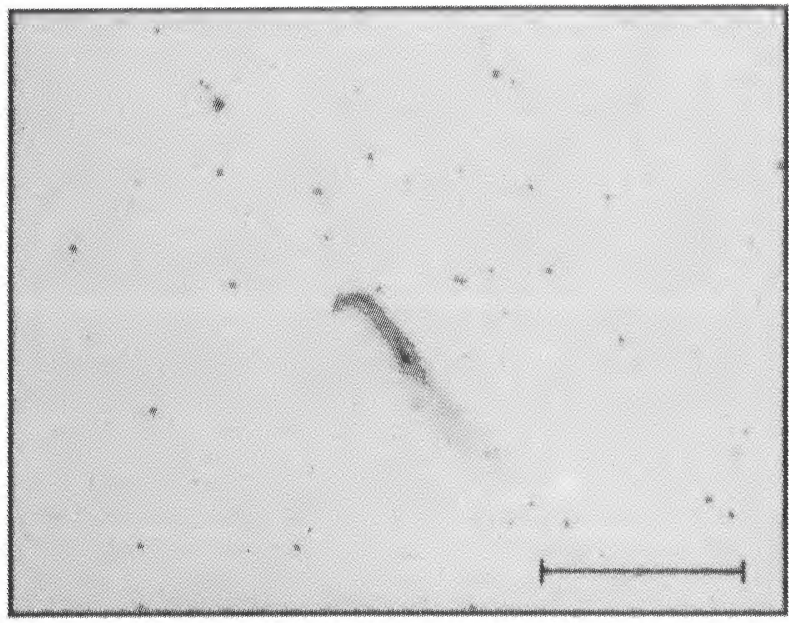

Blue Light

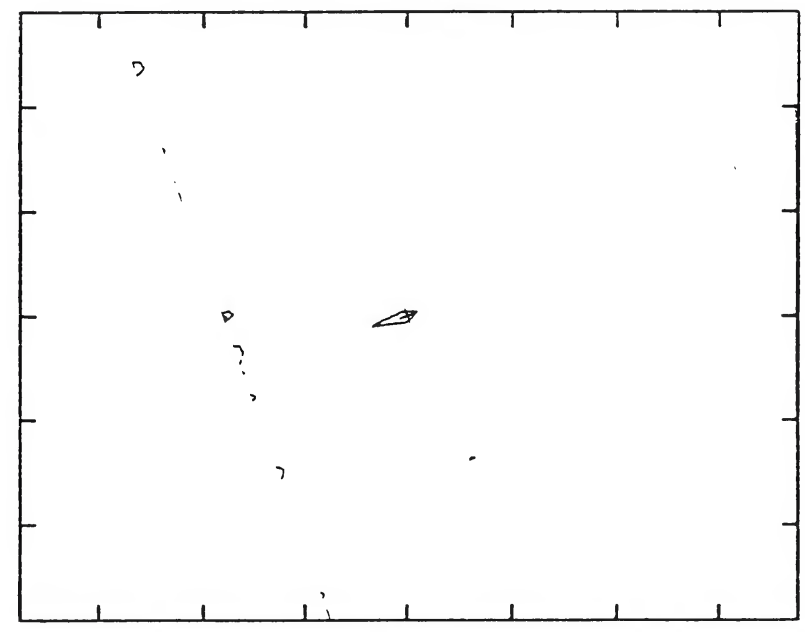

$12 \mu \mathrm{m}$

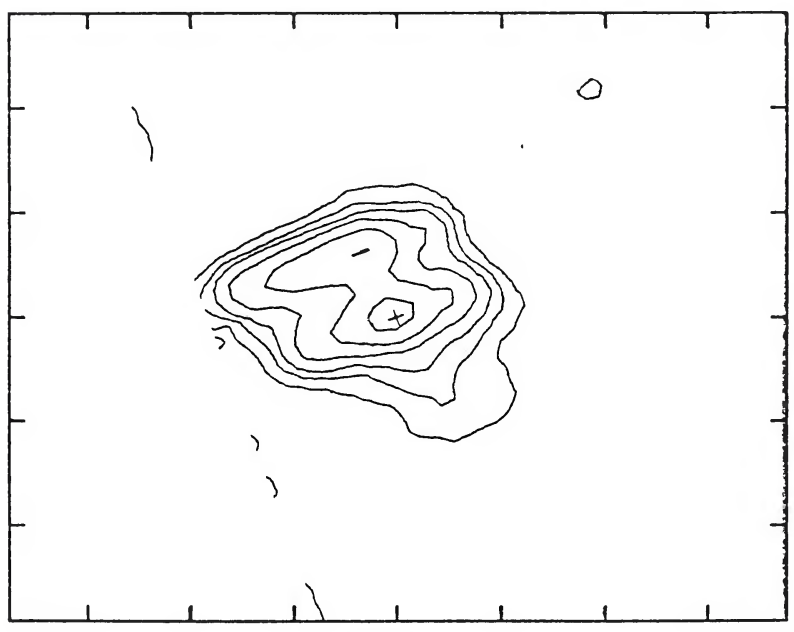

$60 \mu \mathrm{m}$

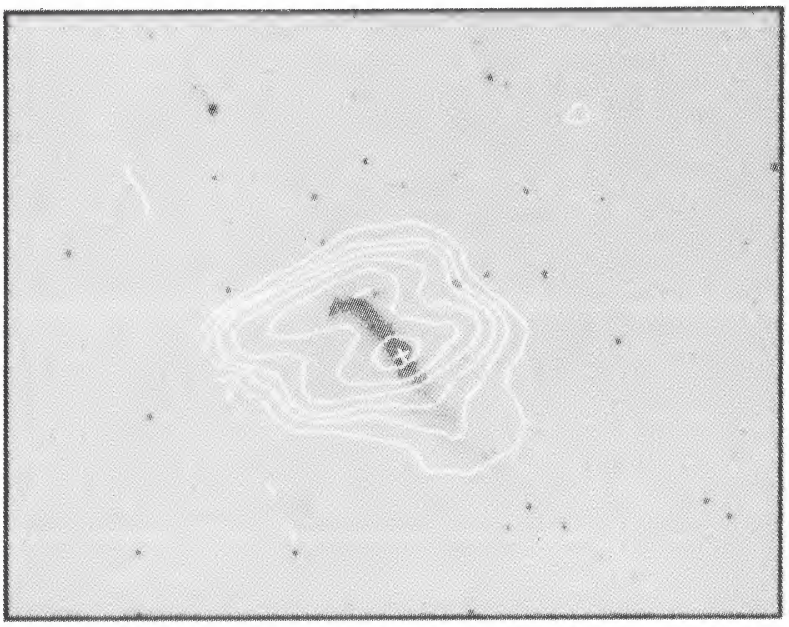

Blue and $60 \mu \mathrm{m}$
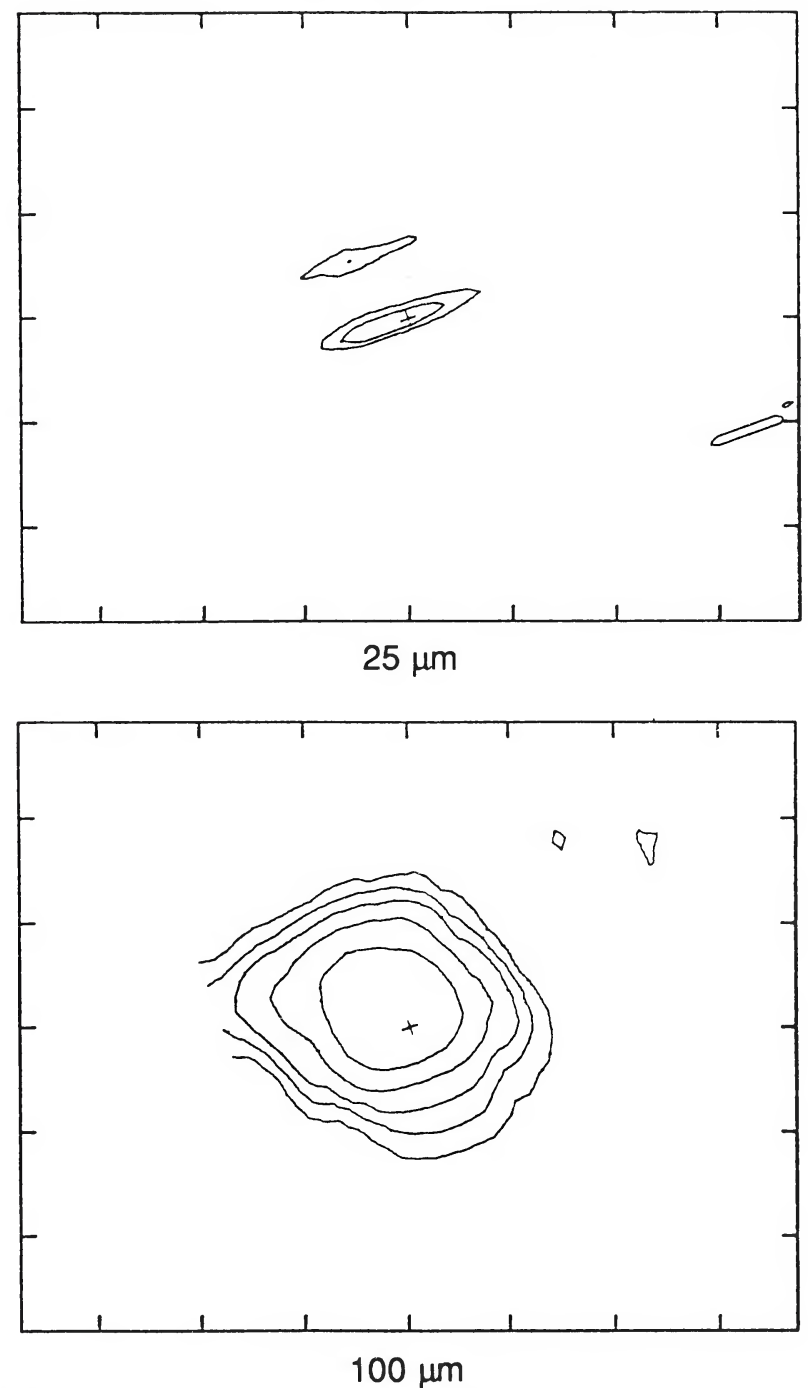

FIG. 24.- NGC 4656. (Optical photographs reproduced by permission of the California Institute of Technology. (c) 1960 National Geographic Society-Palomar Sky Survey.)

RICE et al. (see 68, 102) 


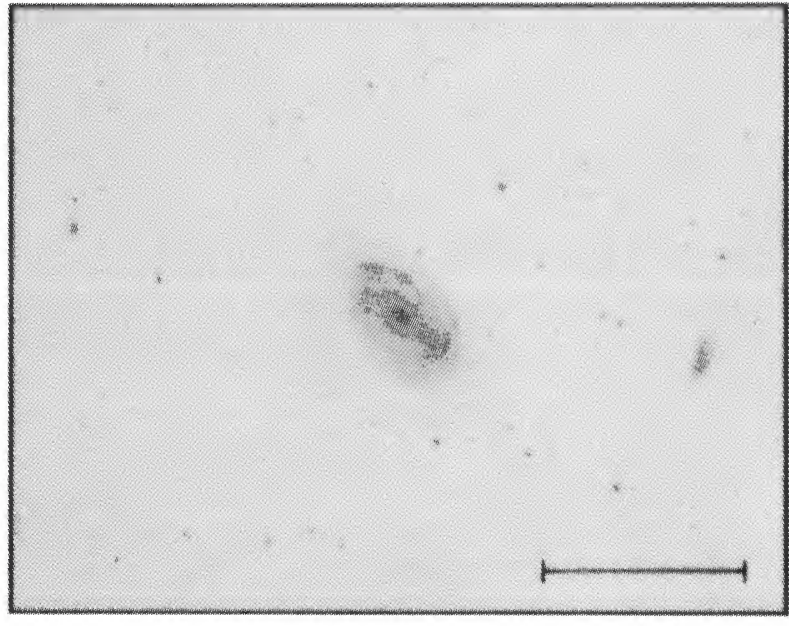

Blue Light
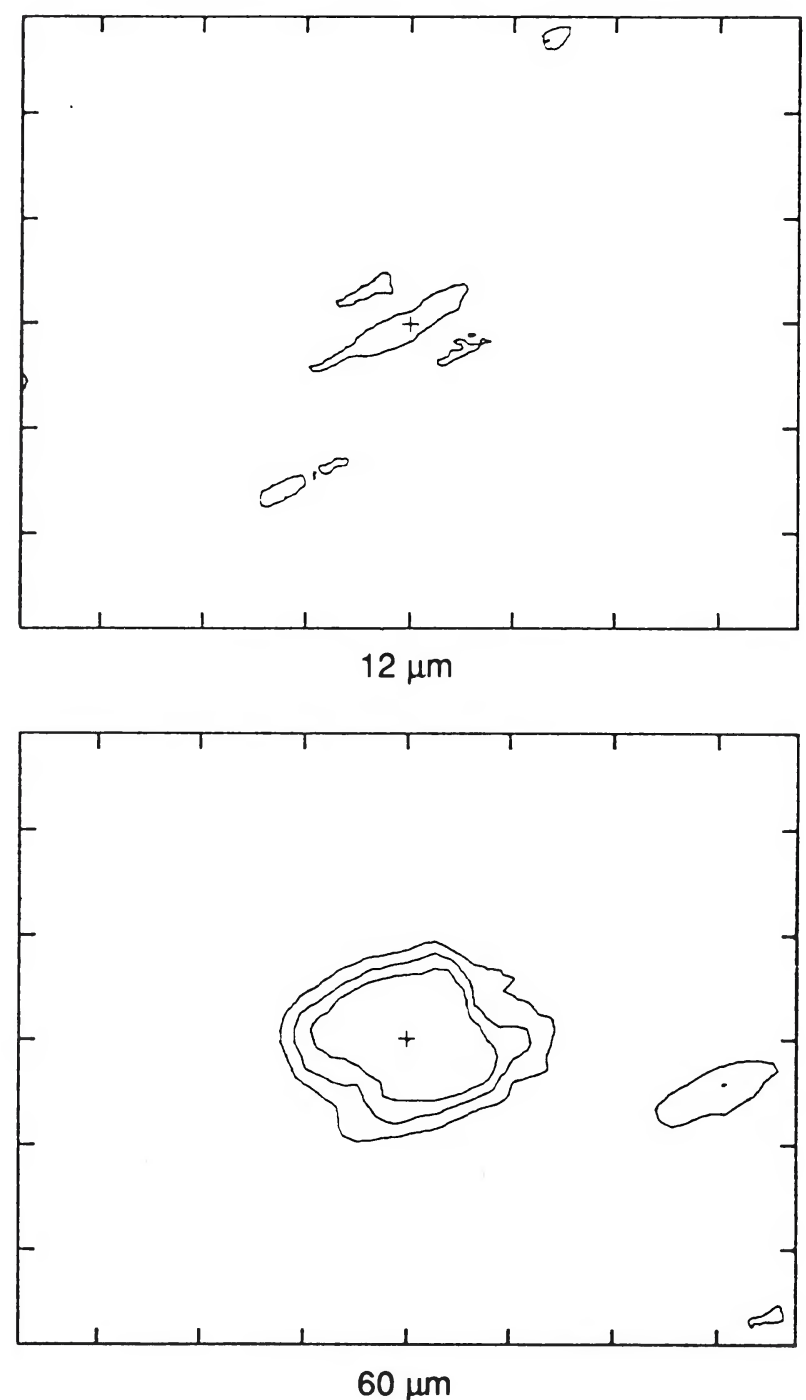

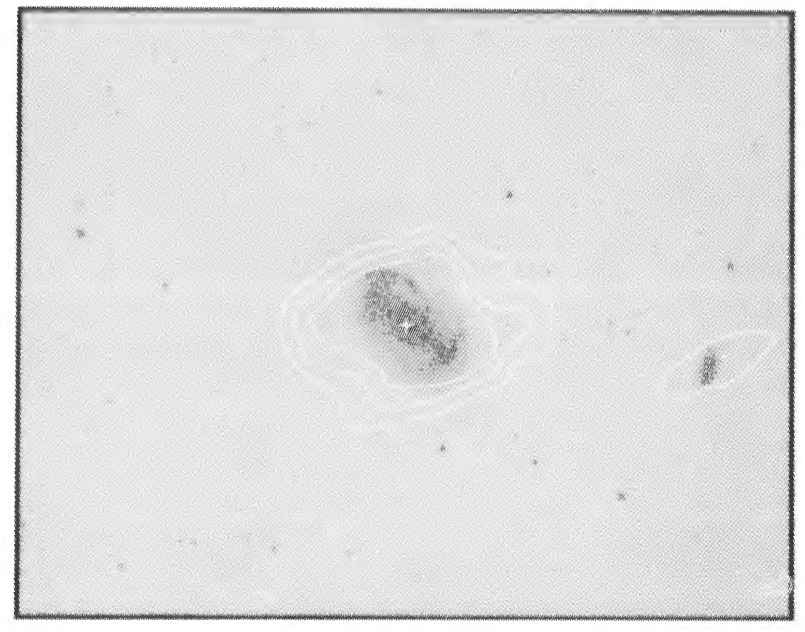

Blue and $60 \mu \mathrm{m}$
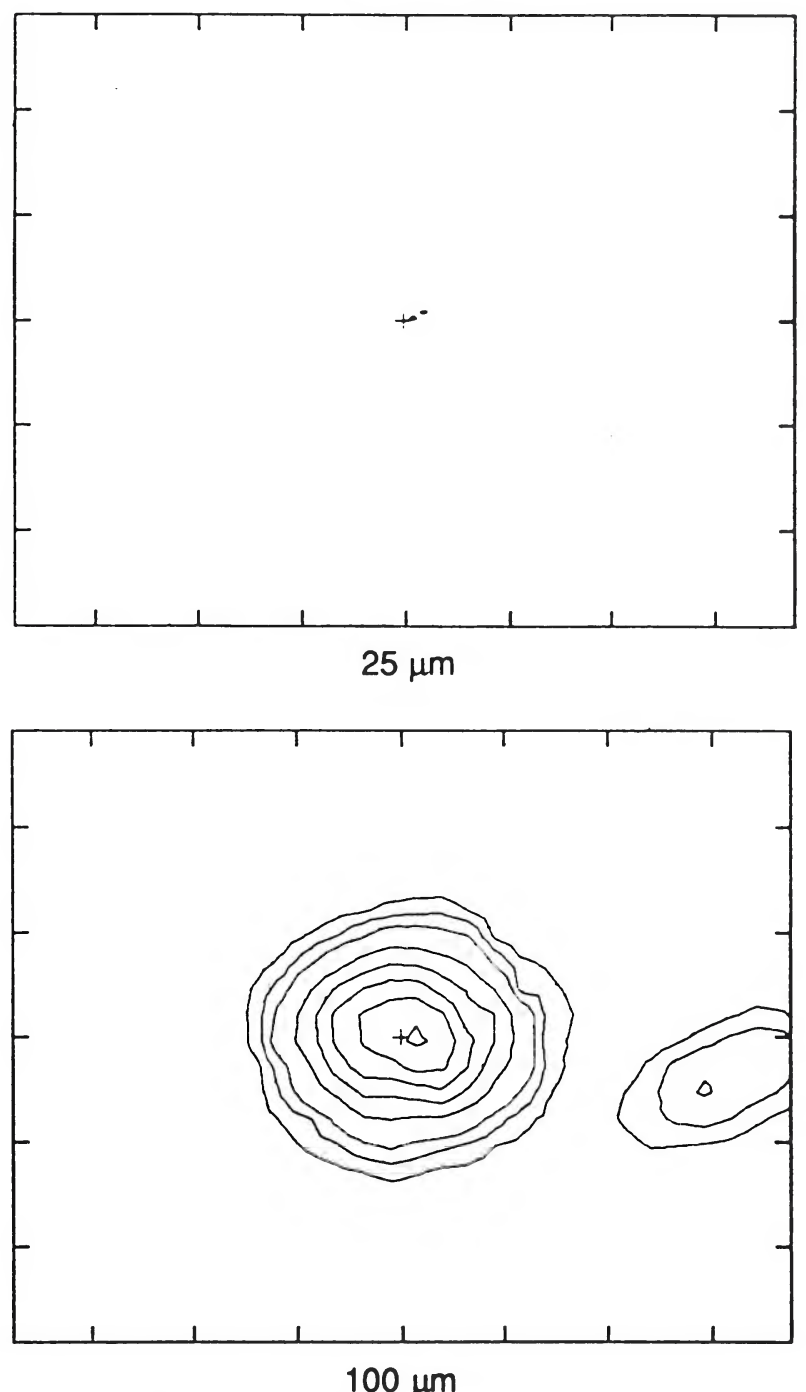

FIG. 25.-NGC 4725. The map contour levels are at (dashed) 3,6, and $9 \sigma$ below the map mean background level and at (solid) 3, 6 , and $10 \sigma$ with brighter levels at increments of $10 \sigma$. (Optical photographs reproduced by permission of the California Institute of Technology. (c) 1960 National Geographic Society-Palomar Sky Survey.)

Rice et al. (see 68, 102) 


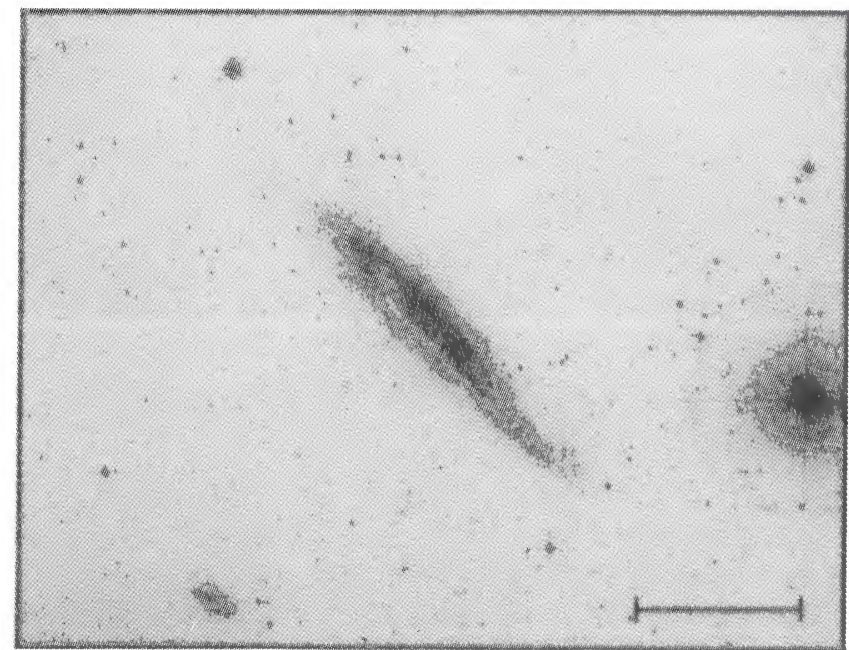

Blue Light
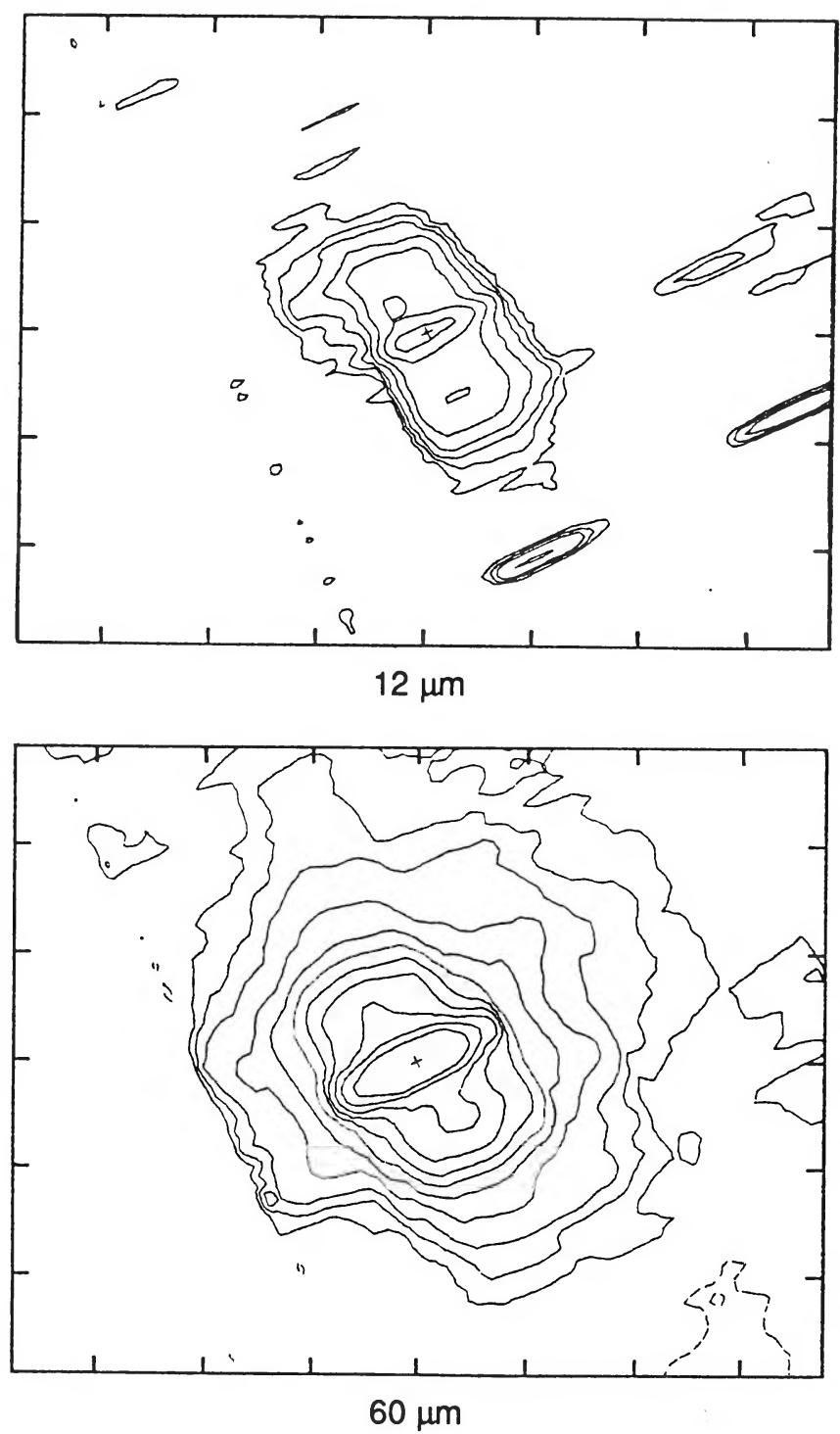

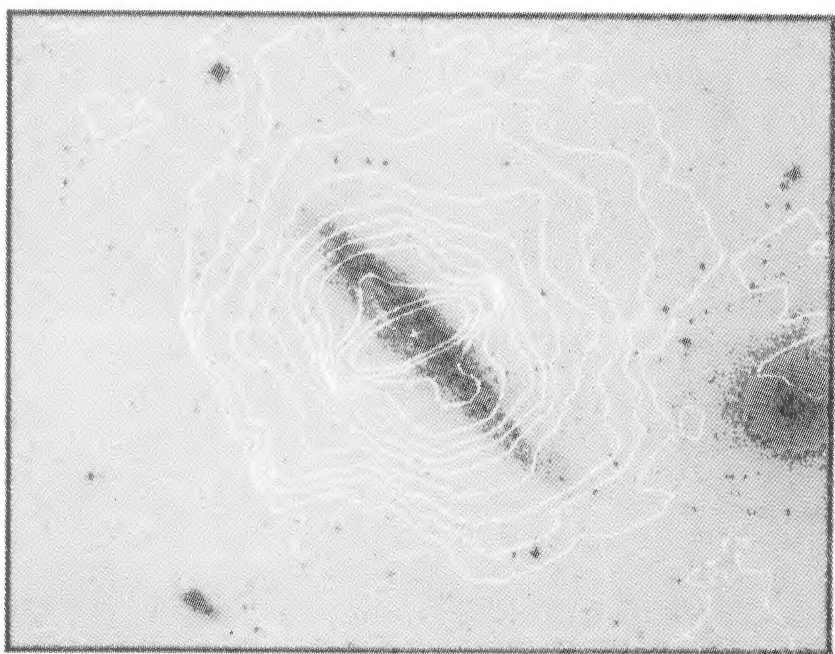

Blue and $60 \mu \mathrm{m}$

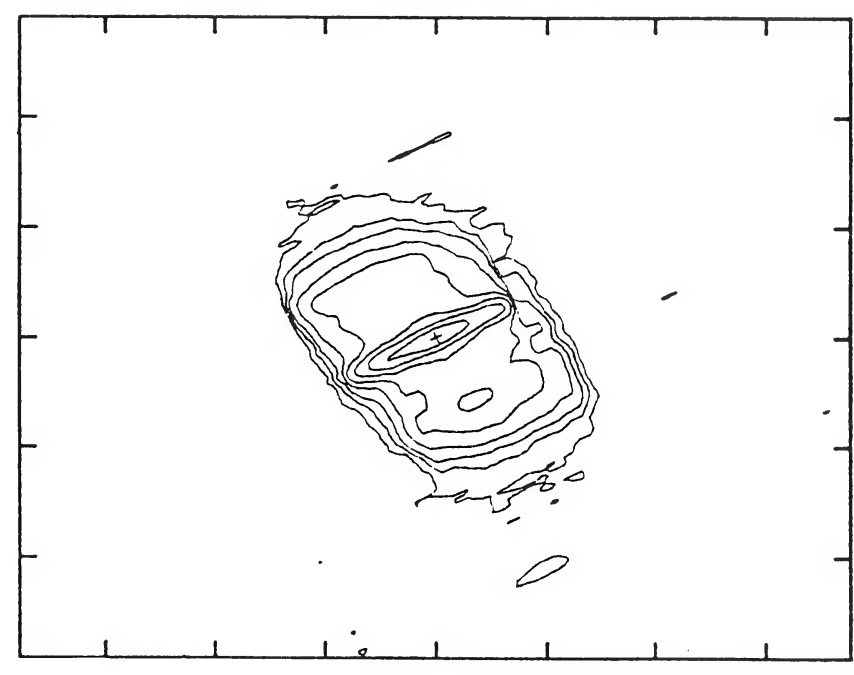

$25 \mu \mathrm{m}$

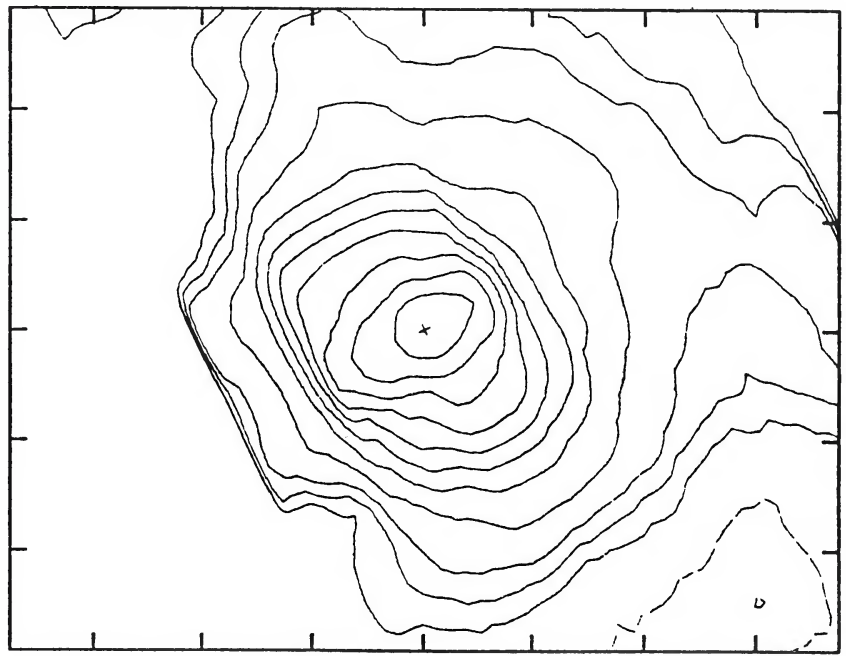

$100 \mu \mathrm{m}$

FIG. 26. - NGC 4945. The tick marks on the infrared maps are spaced by 5'4. (Optical photographs reproduced by permission of the European Southern Observatory.)

Rice et al. (see 68, 102) 


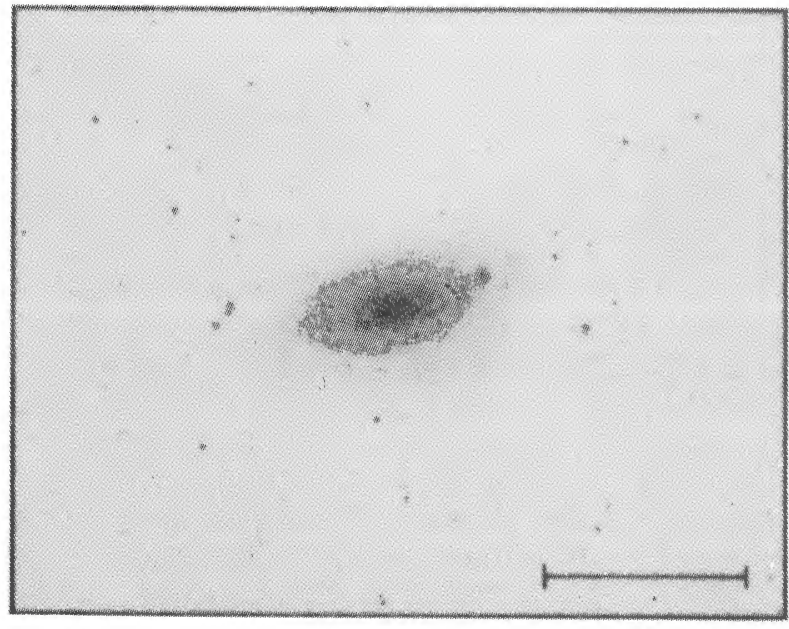

Blue Light
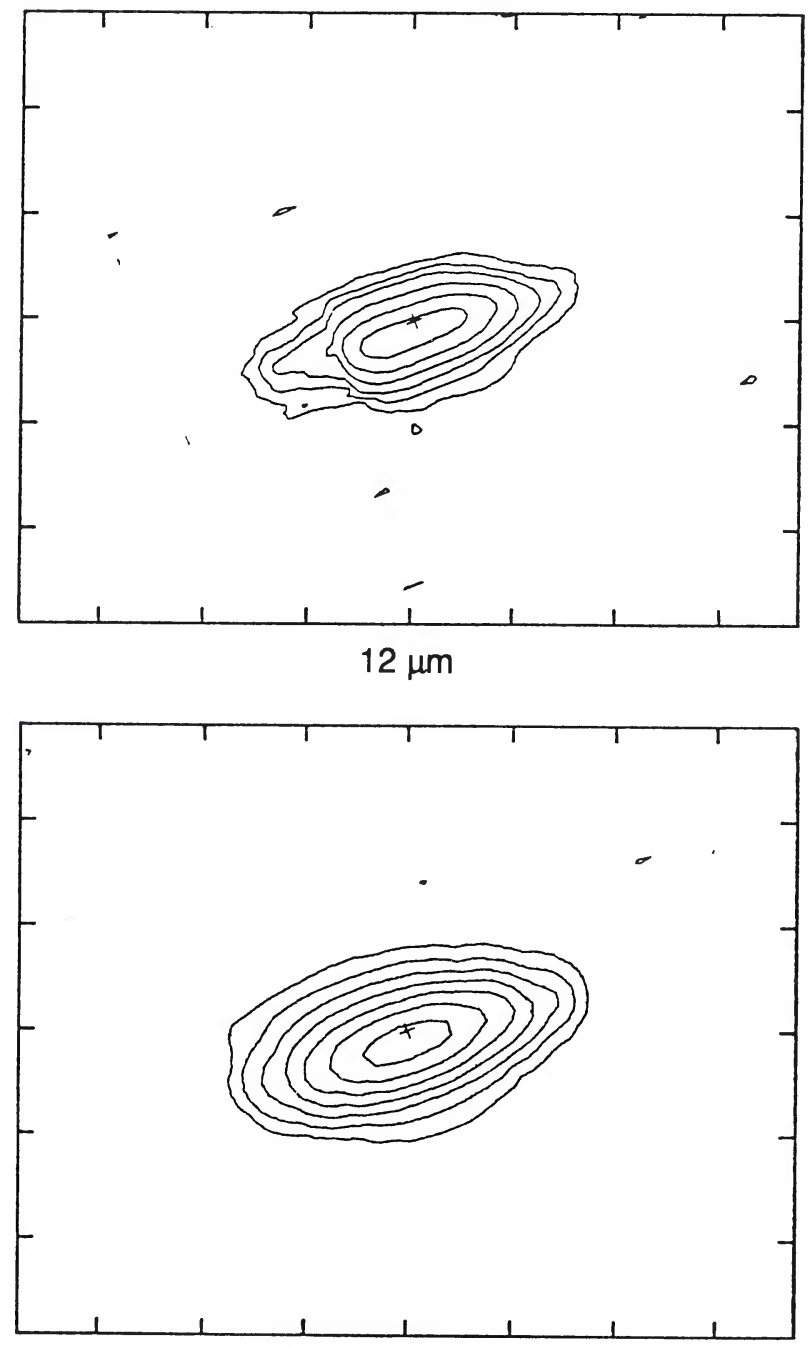

$60 \mu \mathrm{m}$

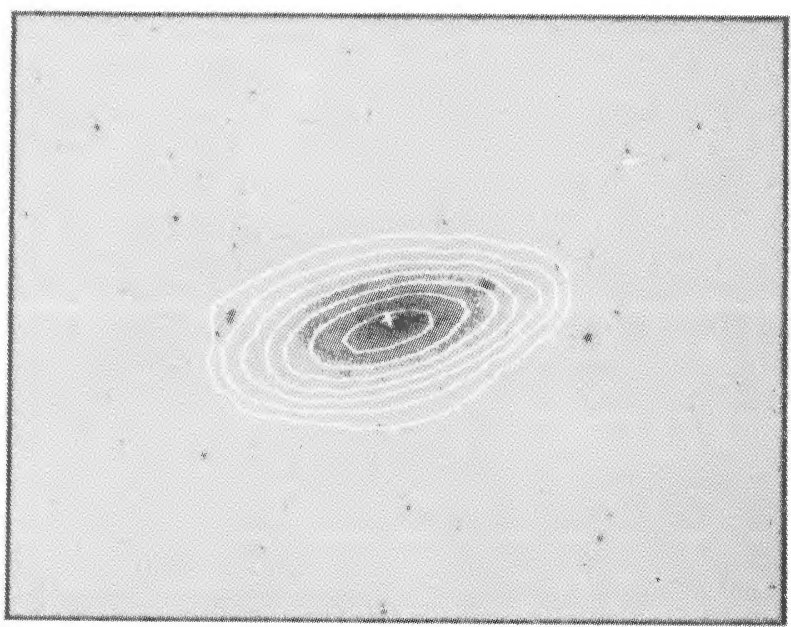

Blue and $60 \mu \mathrm{m}$
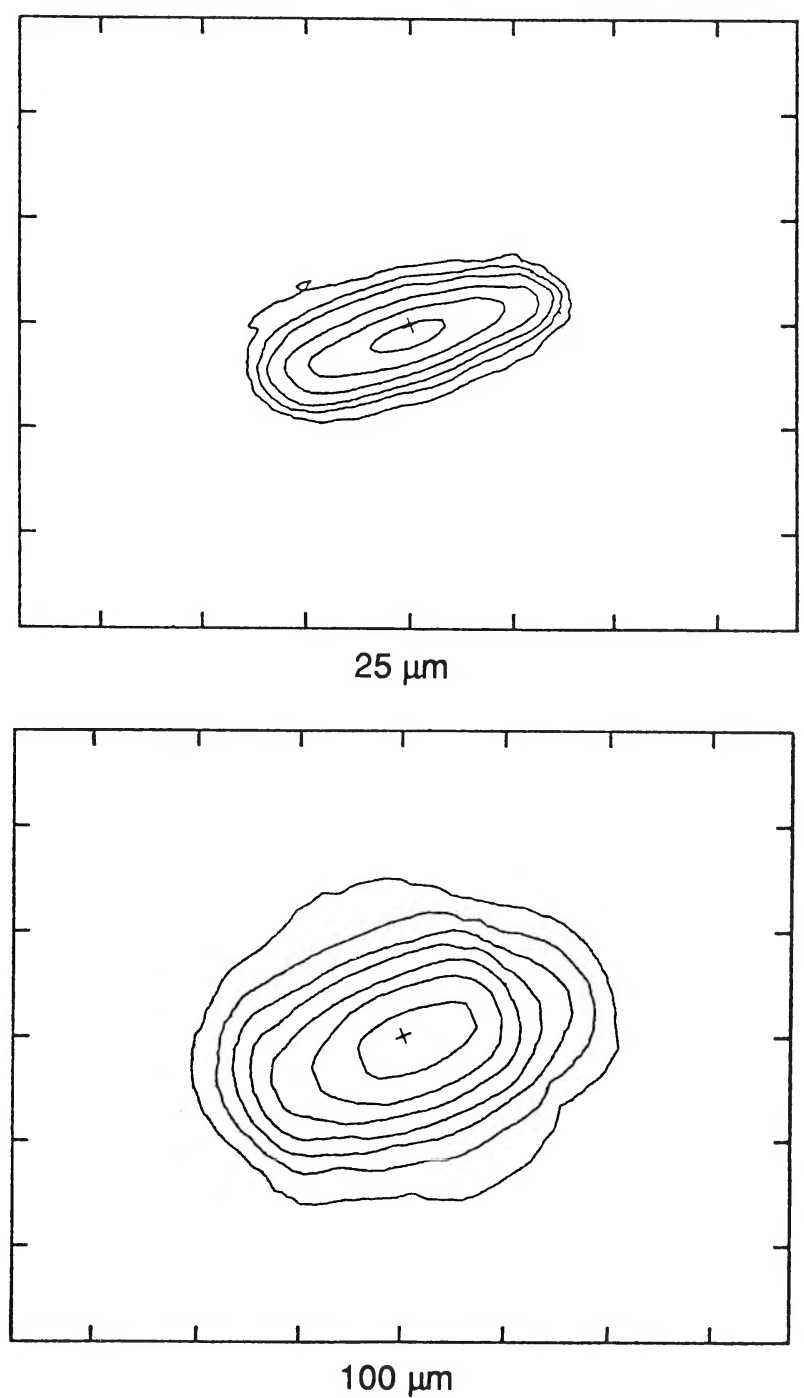

FIG. 27. - NGC 5055. The map contour levels are at (dashed) 3, 6, and $9 \sigma$ below the map mean background level and at (solid) $10 \sigma$ with brighter levels at increments of $10 \sigma$. (Optical photographs reproduced by permission of the California Institute of Technology. (c) 1960 National Geographic Society-Palomar Sky Survey.)

Rice et al. (see 68, 102) 


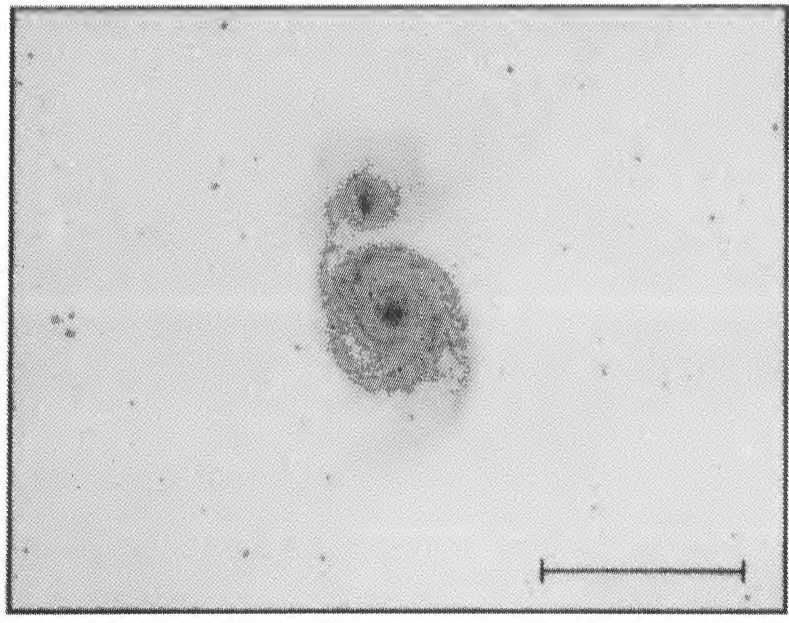

Blue Light
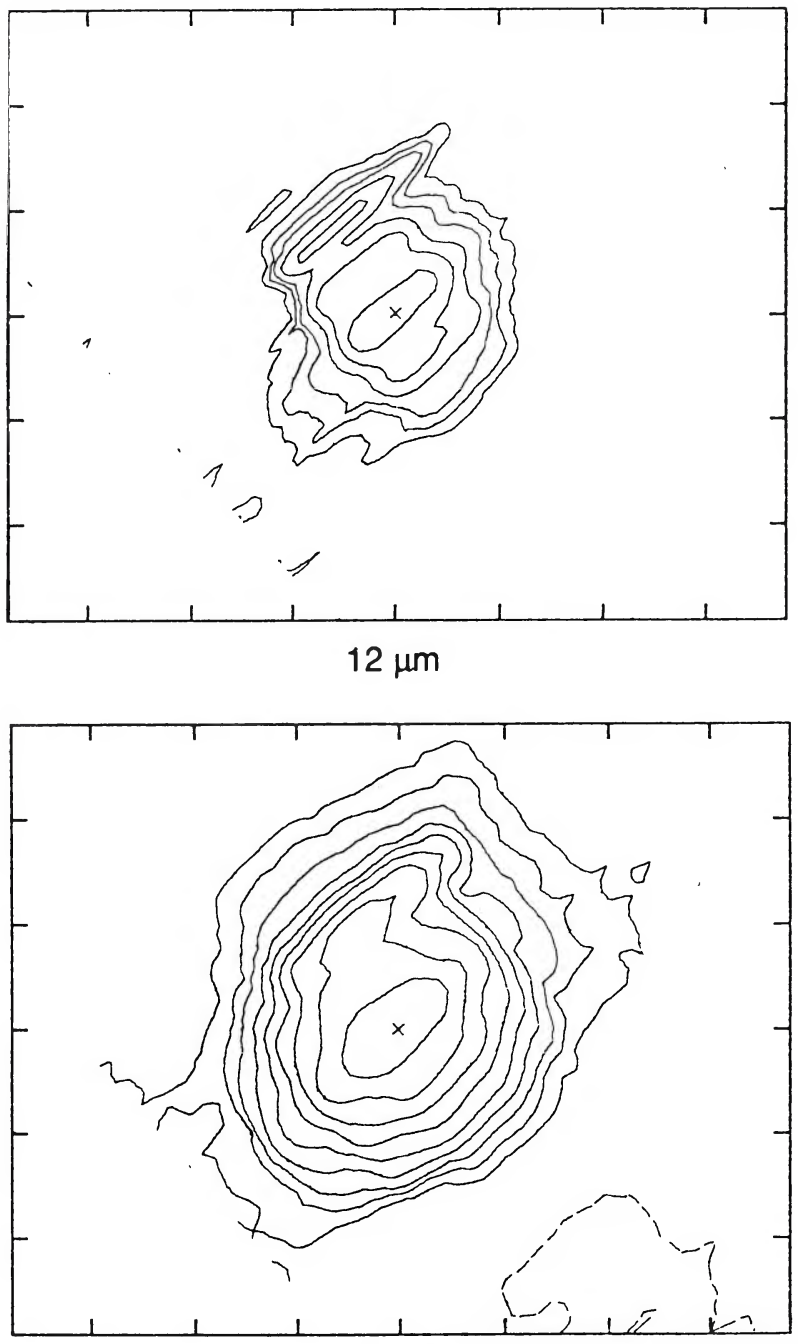

$60 \mu \mathrm{m}$

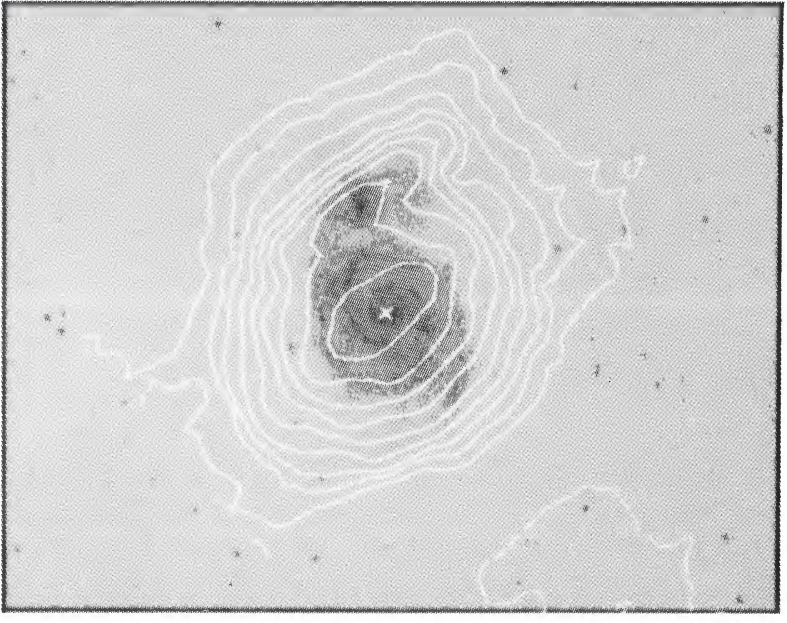

Blue and $60 \mu \mathrm{m}$

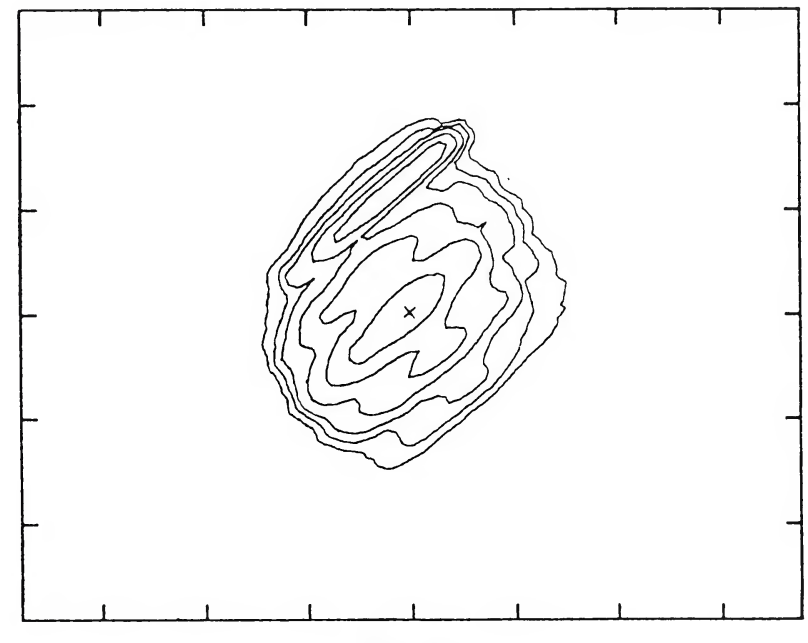

$25 \mu \mathrm{m}$

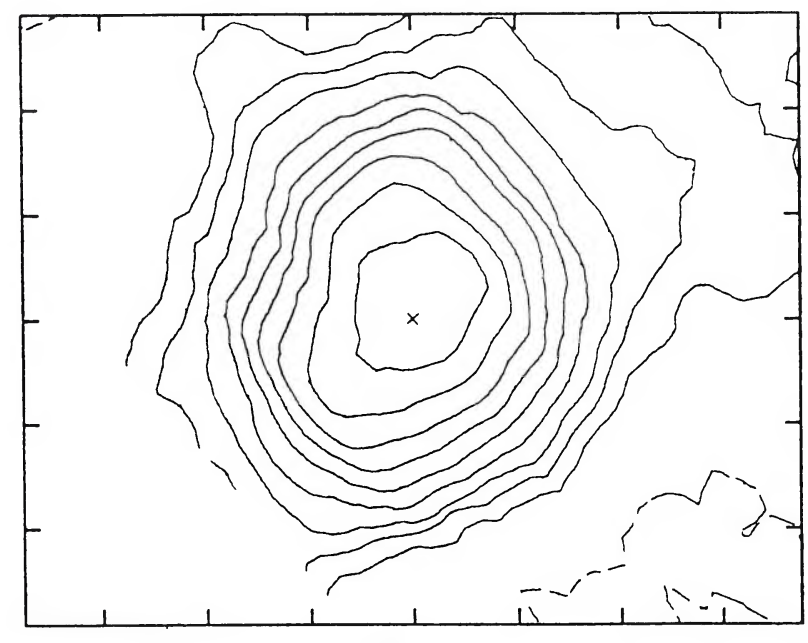

$100 \mu \mathrm{m}$

FIG. 28. - NGC 5194. (Optical photographs reproduced by permission of the California Institute of Technology. (C) 1960 National Geographic Society-Palomar Sky Survey.)

Rice et al. (see 68, 102) 
0
0
-1
$\vdots$
0
0
0
$\vdots$
0
2
0
1
0
0
0
0
-1

PLATE 164

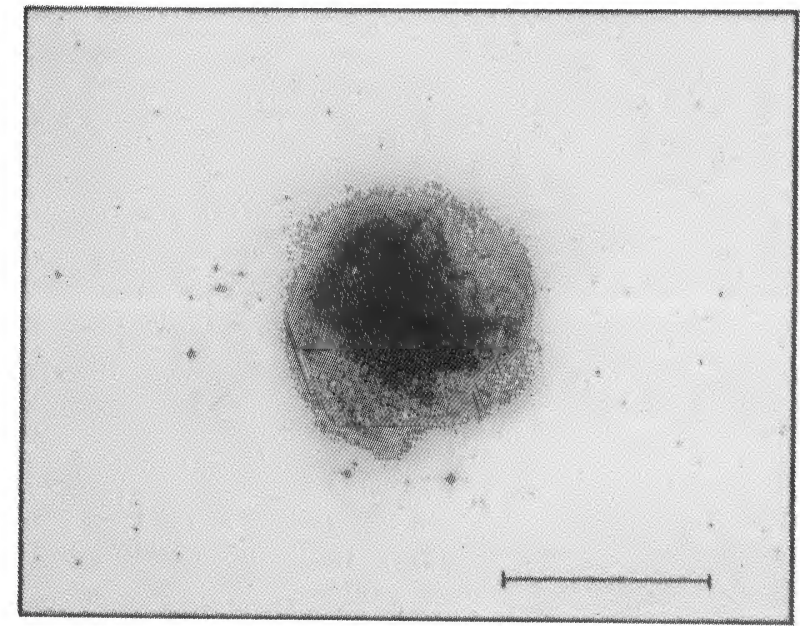

Blue Light

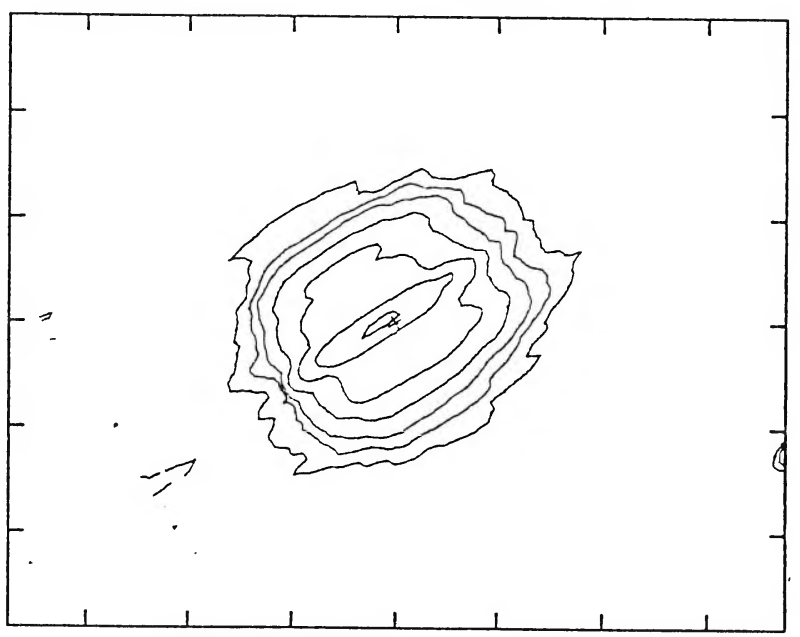

$12 \mu \mathrm{m}$

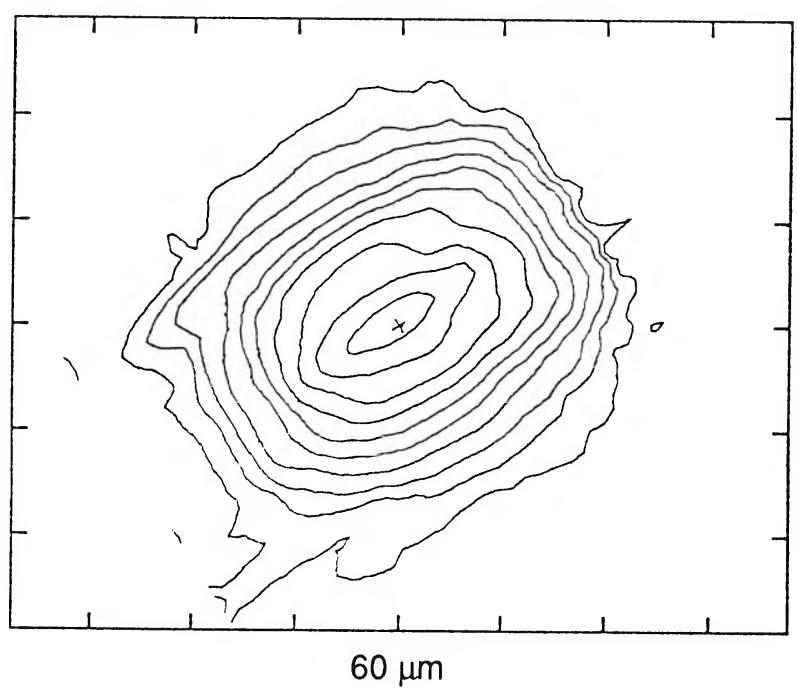

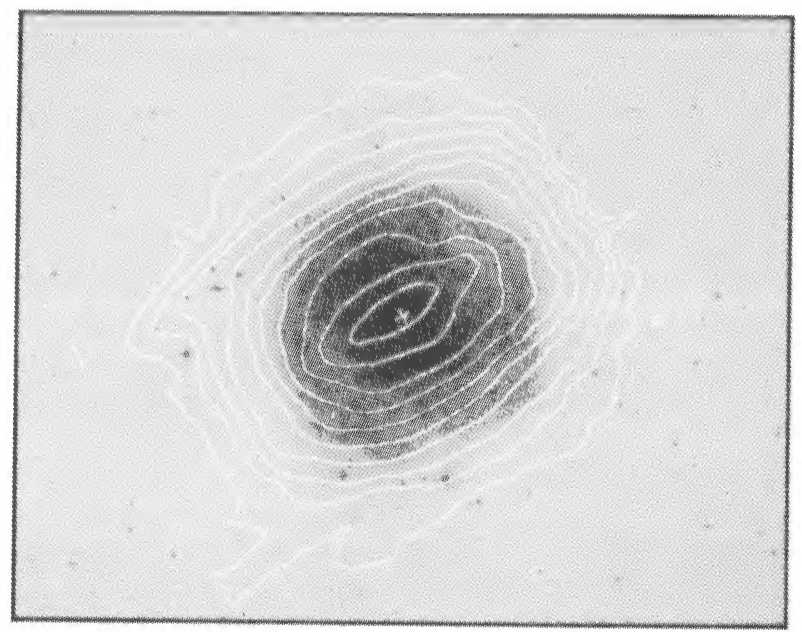

Blue and $60 \mu \mathrm{m}$
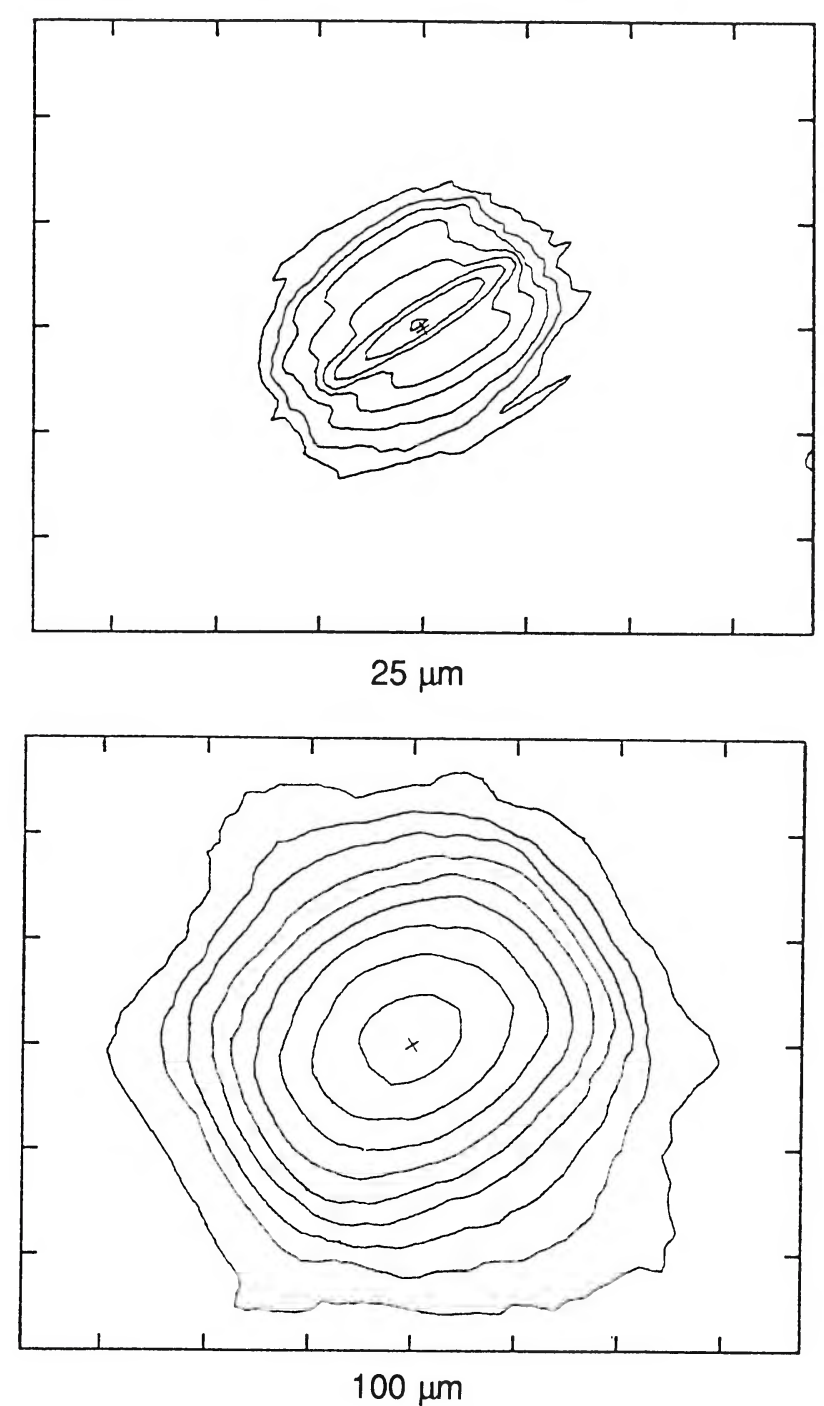

FIG. 29. - NGC 5236. (Optical photographs reproduced by permission of the European Southern Observatory.)

RICE et al. (see 68, 102) 


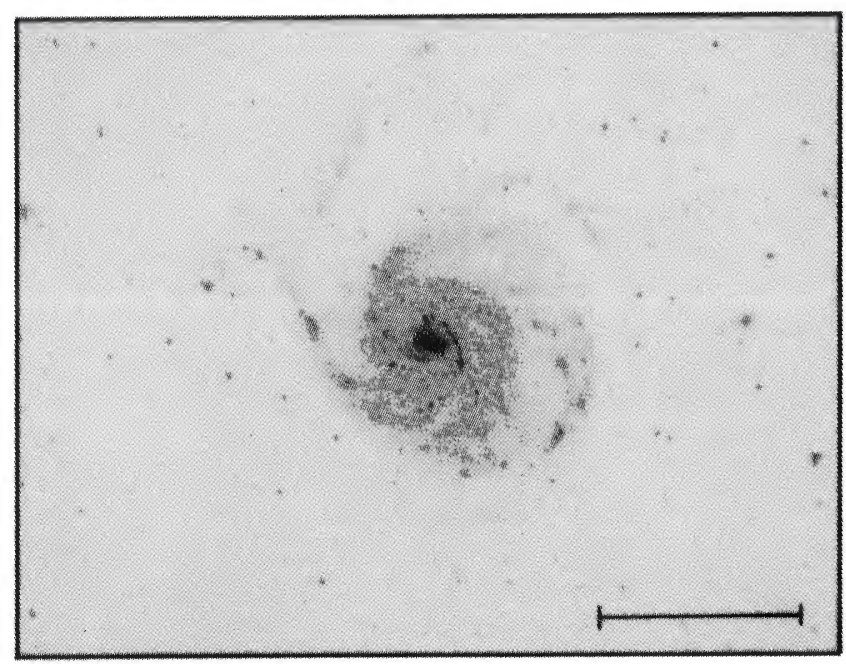

Blue Light
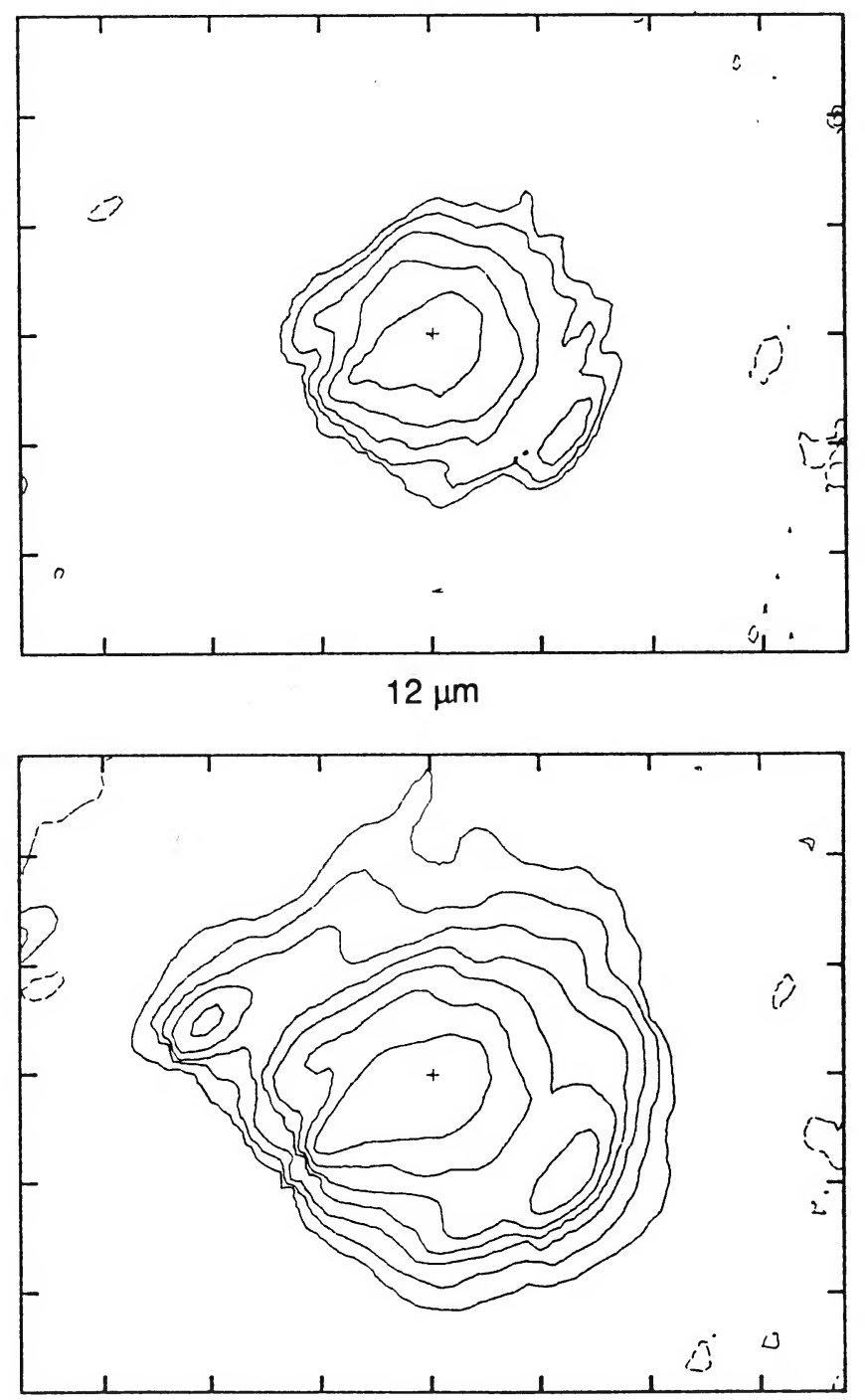

$60 \mu \mathrm{m}$

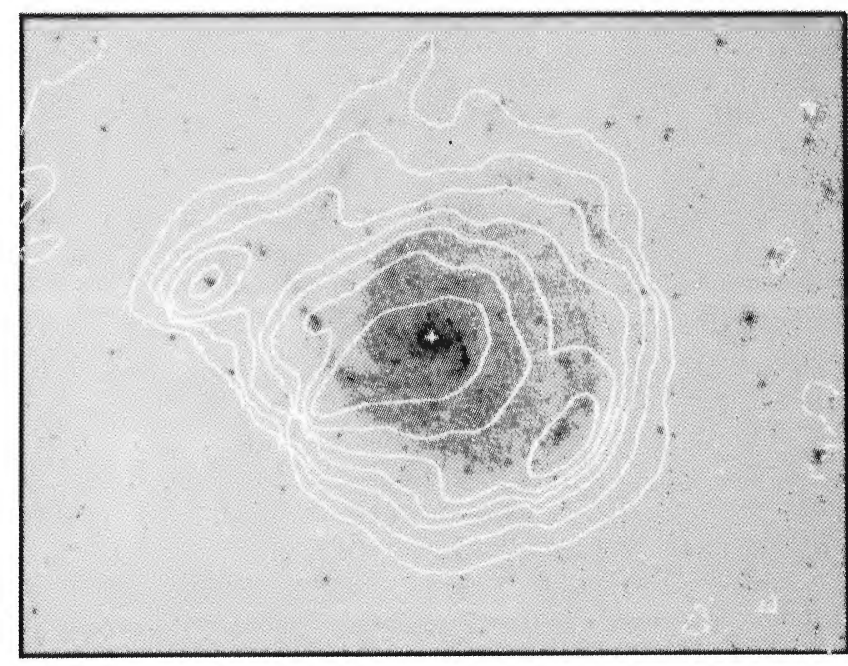

Blue and $60 \mu \mathrm{m}$

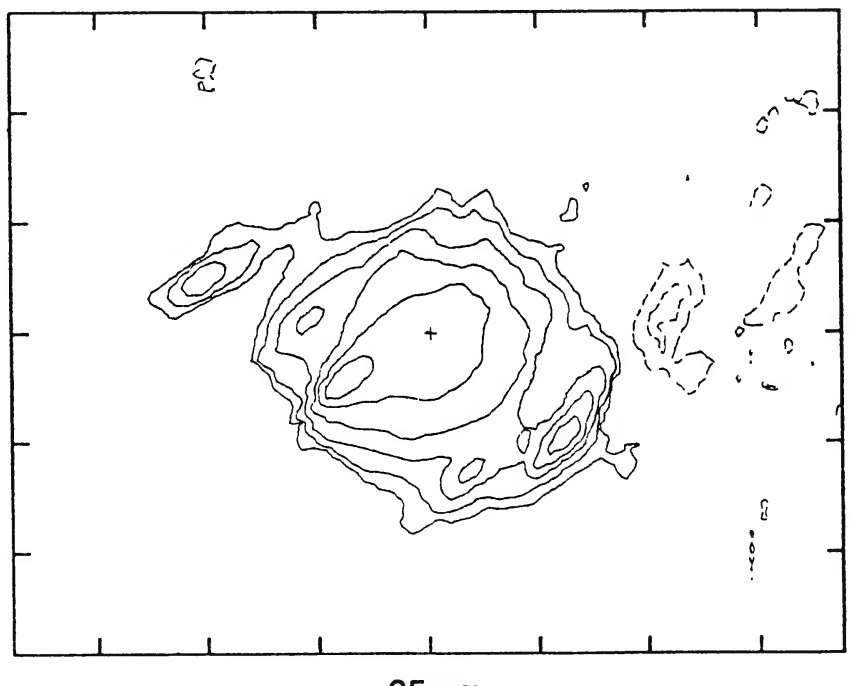

$25 \mu \mathrm{m}$

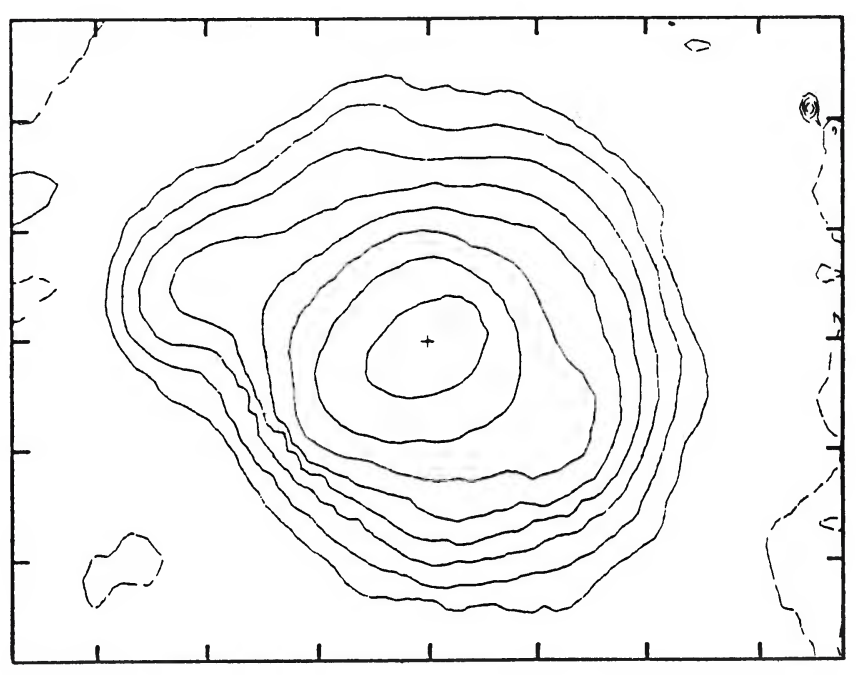

$100 \mu \mathrm{m}$

FIg. 30.-NGC 5457. The map contours are at (dashed) 3,6, and $9 \sigma$ below the map mean background level and at (solid) 6 and $10 \sigma$ for the 12 and $25 \mu \mathrm{m}$ map, and $10 \sigma$ for the 60 and $100 \mu \mathrm{m}$ maps with brighter contours scaled logarithmically by 2 from $10 \sigma$. The tick marks on the infrared maps are spaced by 5'.4. (Optical photographs reproduced by permission of the California Institute of Technology. (C) 1960 National Geographic Society-Palomar Sky Survey.) 


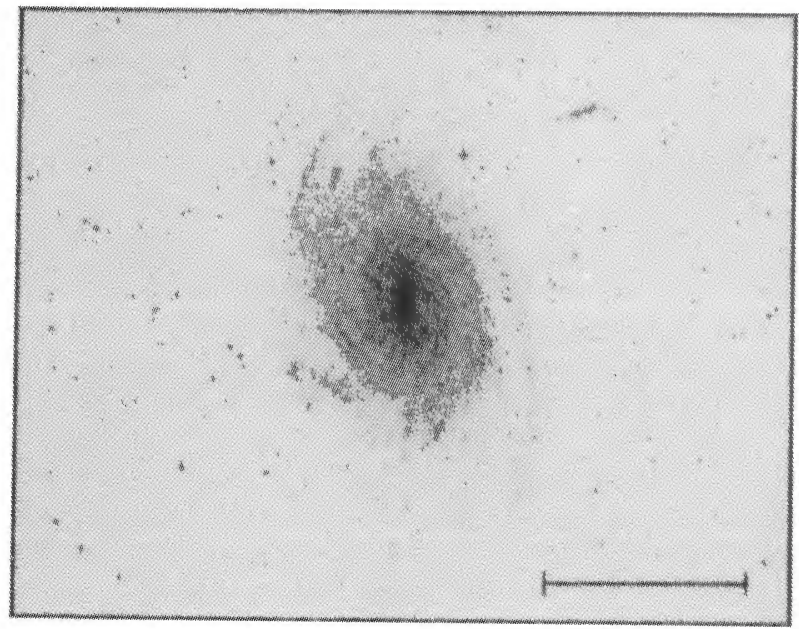

Blue Light
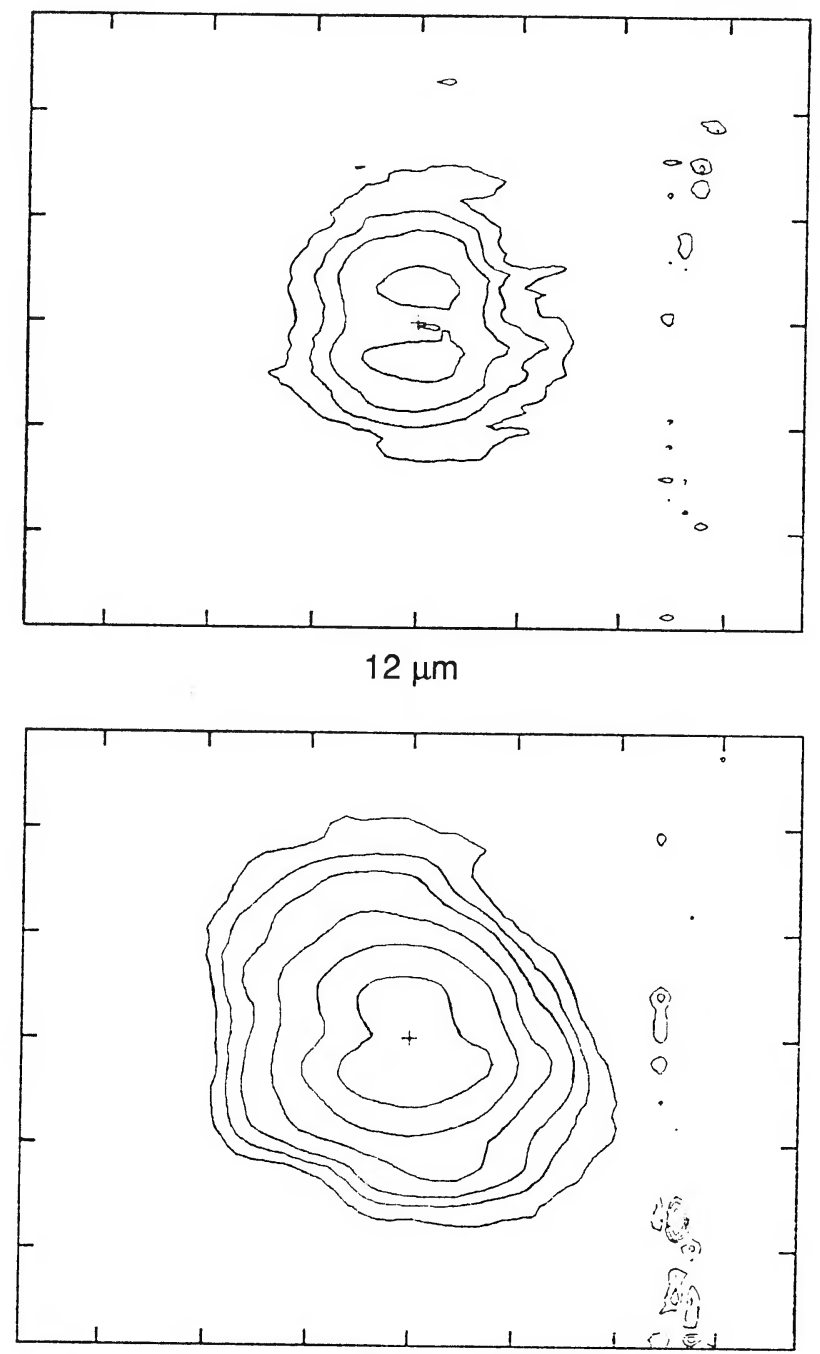

$60 \mu \mathrm{m}$

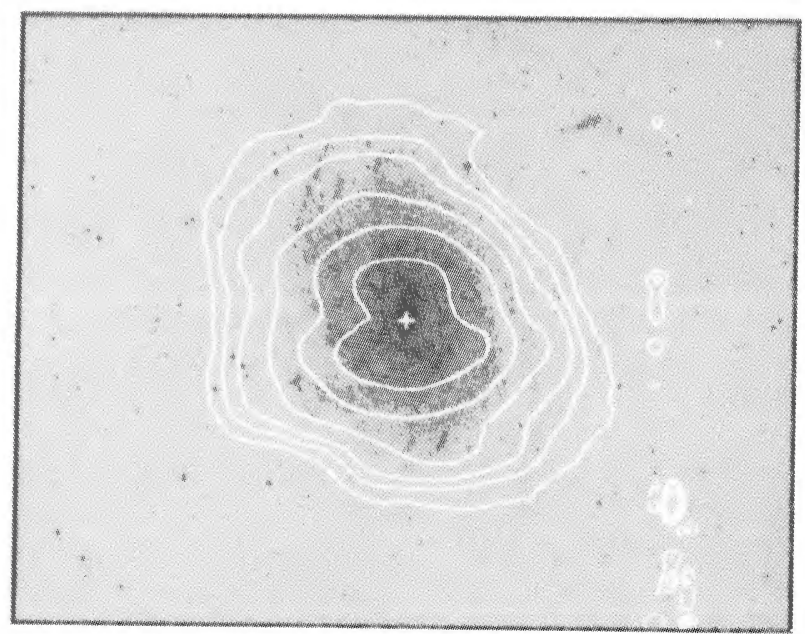

Blue and $60 \mu \mathrm{m}$
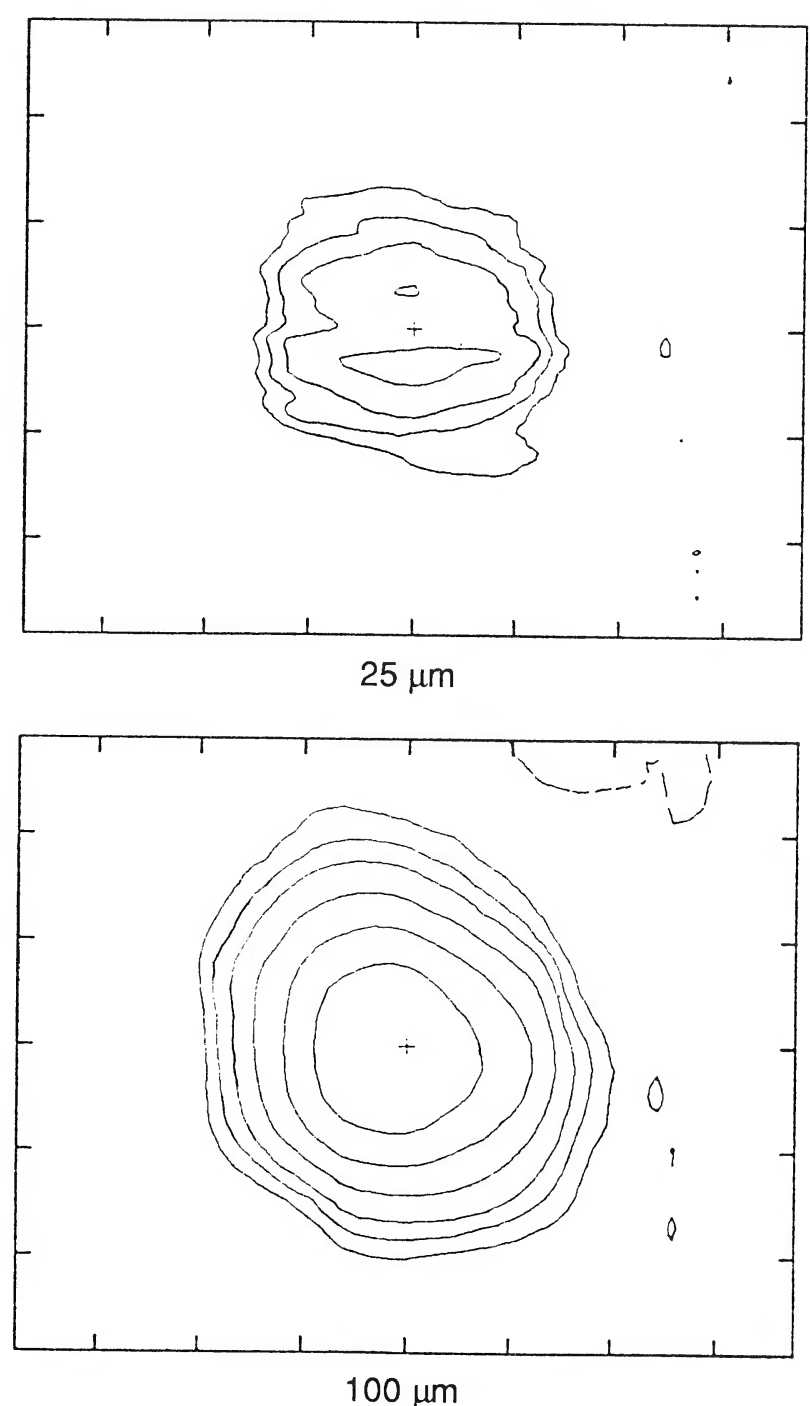

FIG. 31.-NGC 6744. (Optical photographs reproduced by permission of the European Southern Observatory.)

RICE et al. (see 68, 102) 


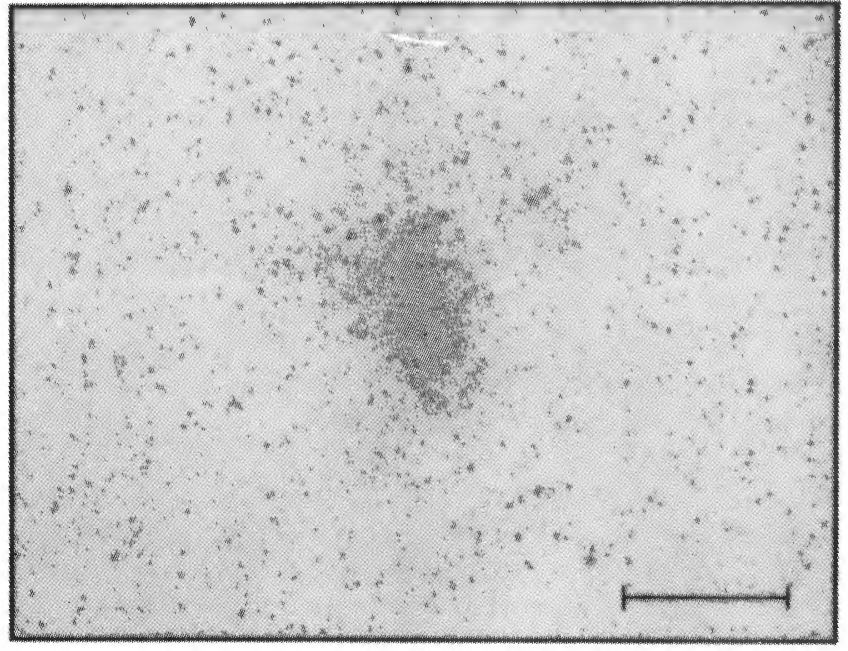

Blue Light
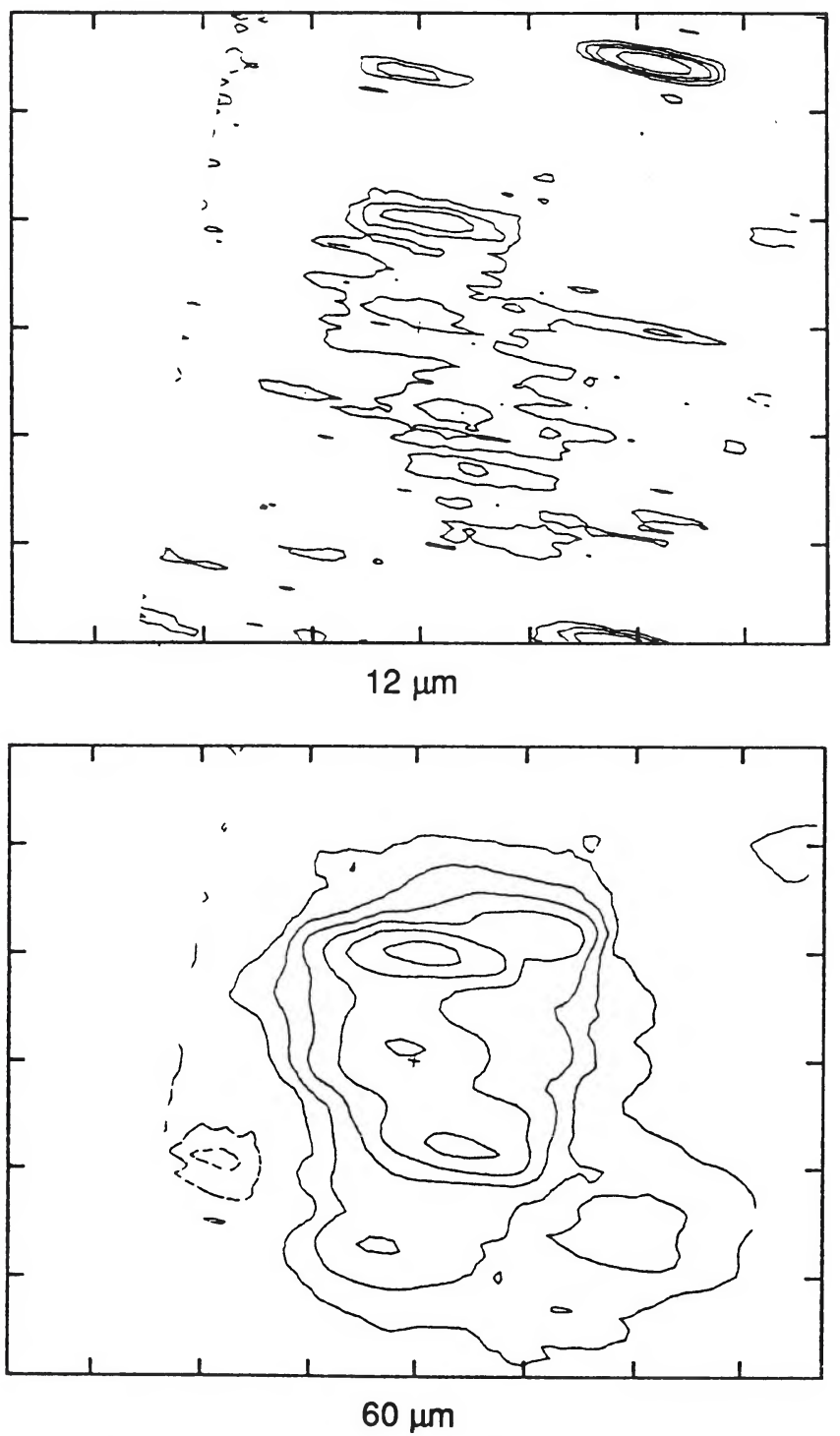

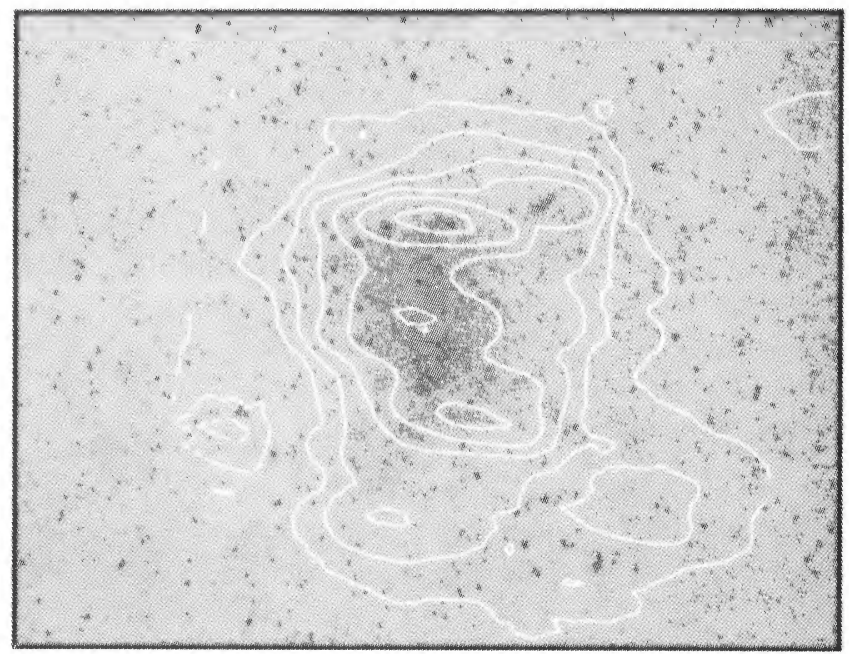

Blue and $60 \mu \mathrm{m}$
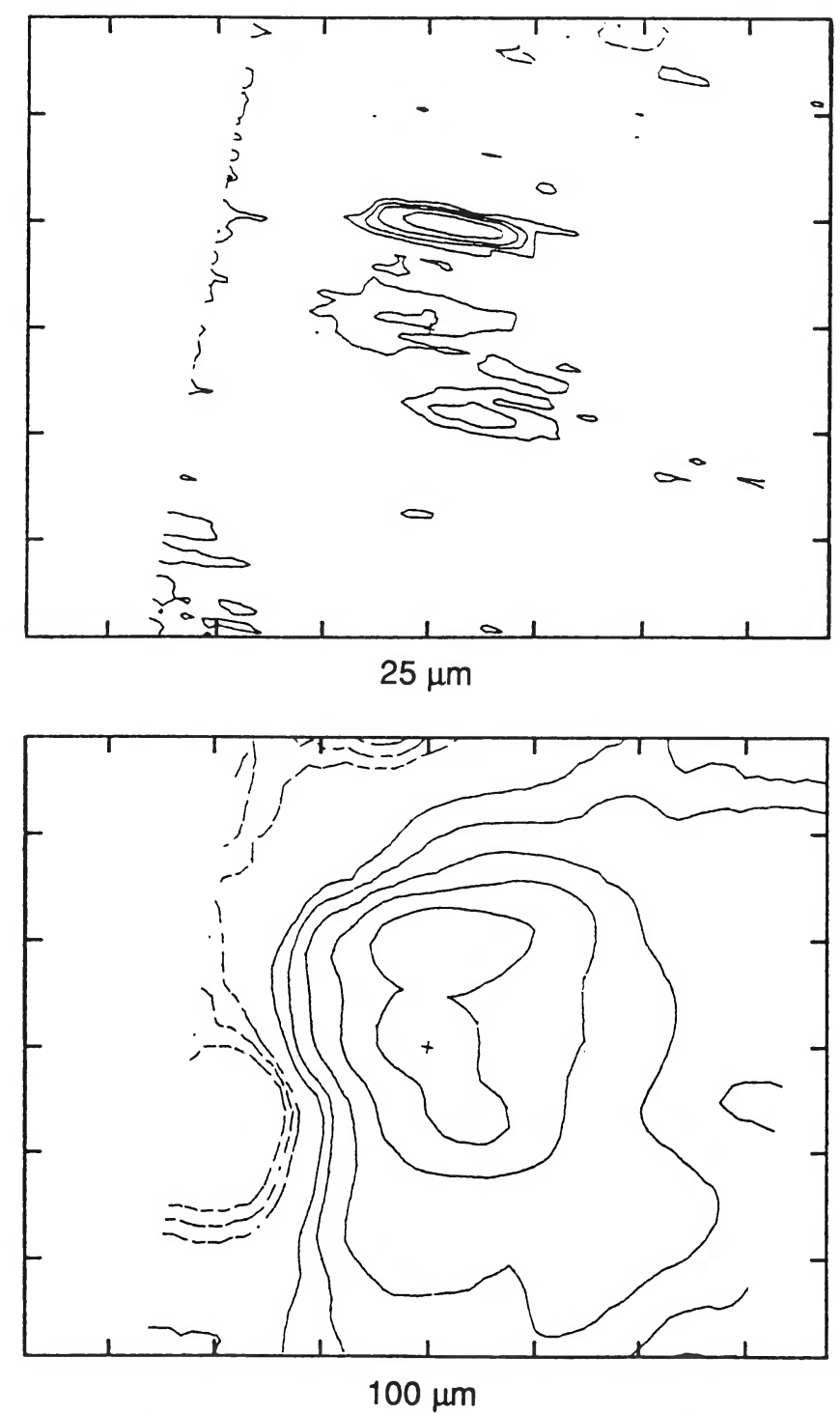

FIG. 32.-NGC 6822. For the 60 and $100 \mu \mathrm{m}$ maps the contours are at (dashed) 3, 6, and $9 \sigma$ below the map mean background level and at (solid) 10 $\sigma$ with brighter contours scaled logarithmically by 2 from $10 \sigma$. The tick marks on the infrared maps are spaced by 5'4. (Optical photographs reproduced by permission of the California Institute of Technology. (C) 1960 National Geographic Society-Palomar Sky Survey.)

Rice et al. (see 68, 102) 


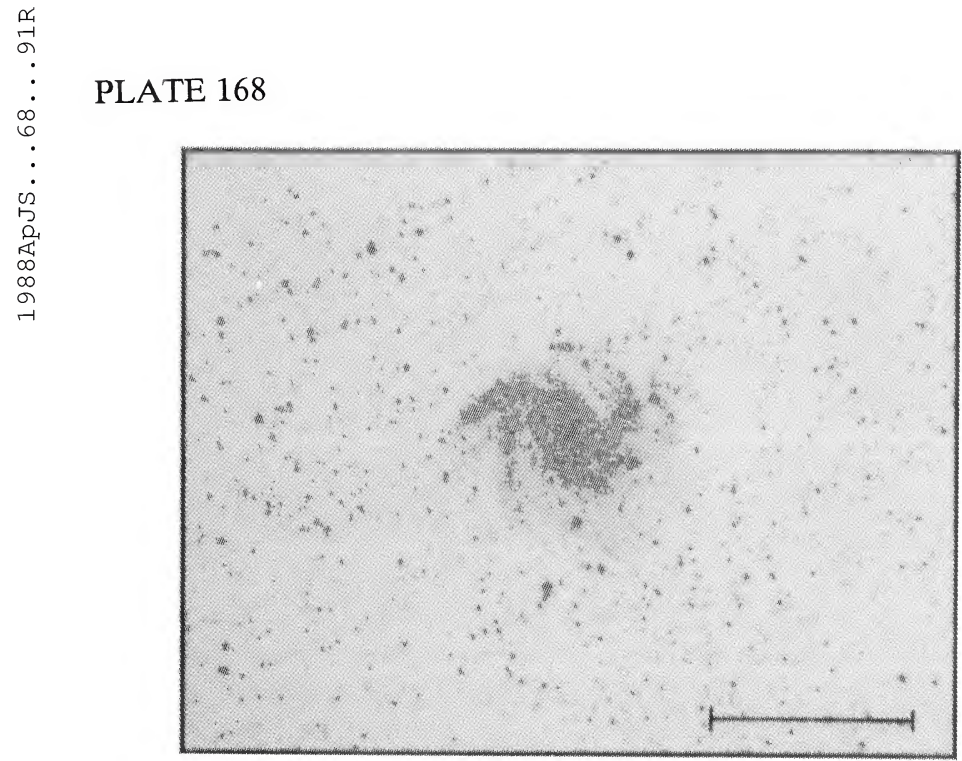

Blue Light

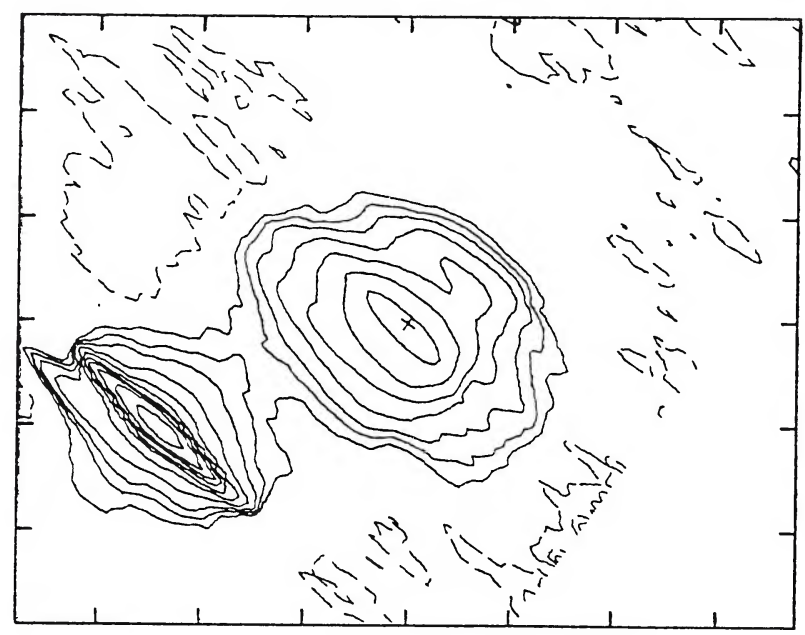

$12 \mu \mathrm{m}$

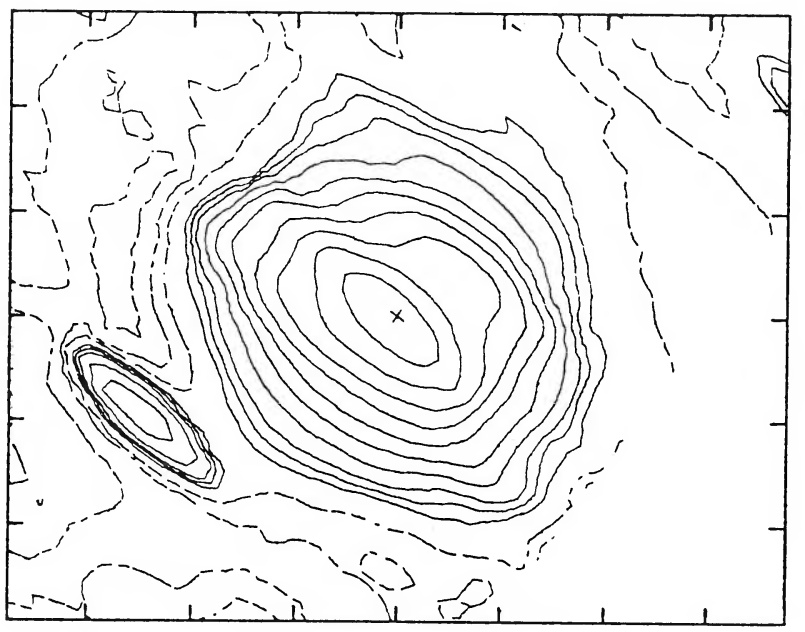

$60 \mu \mathrm{m}$

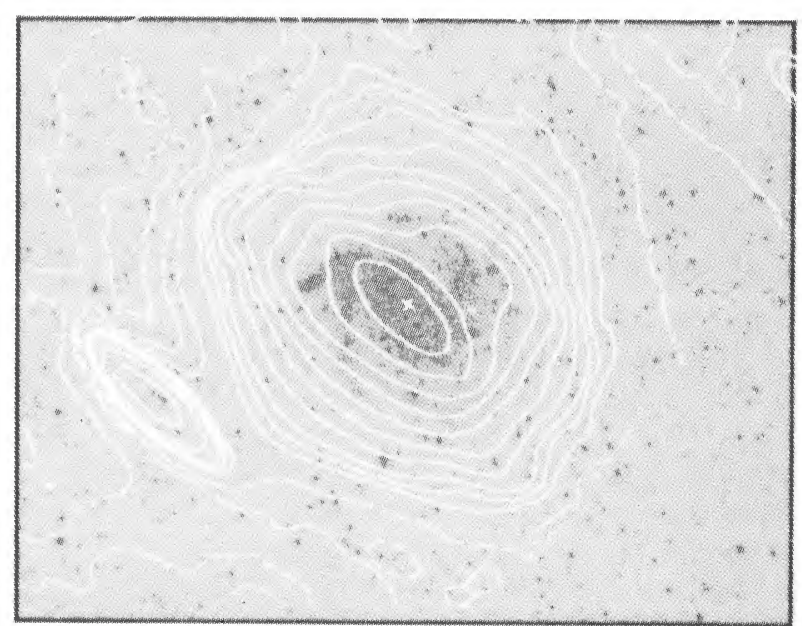

Blue and $60 \mu \mathrm{m}$
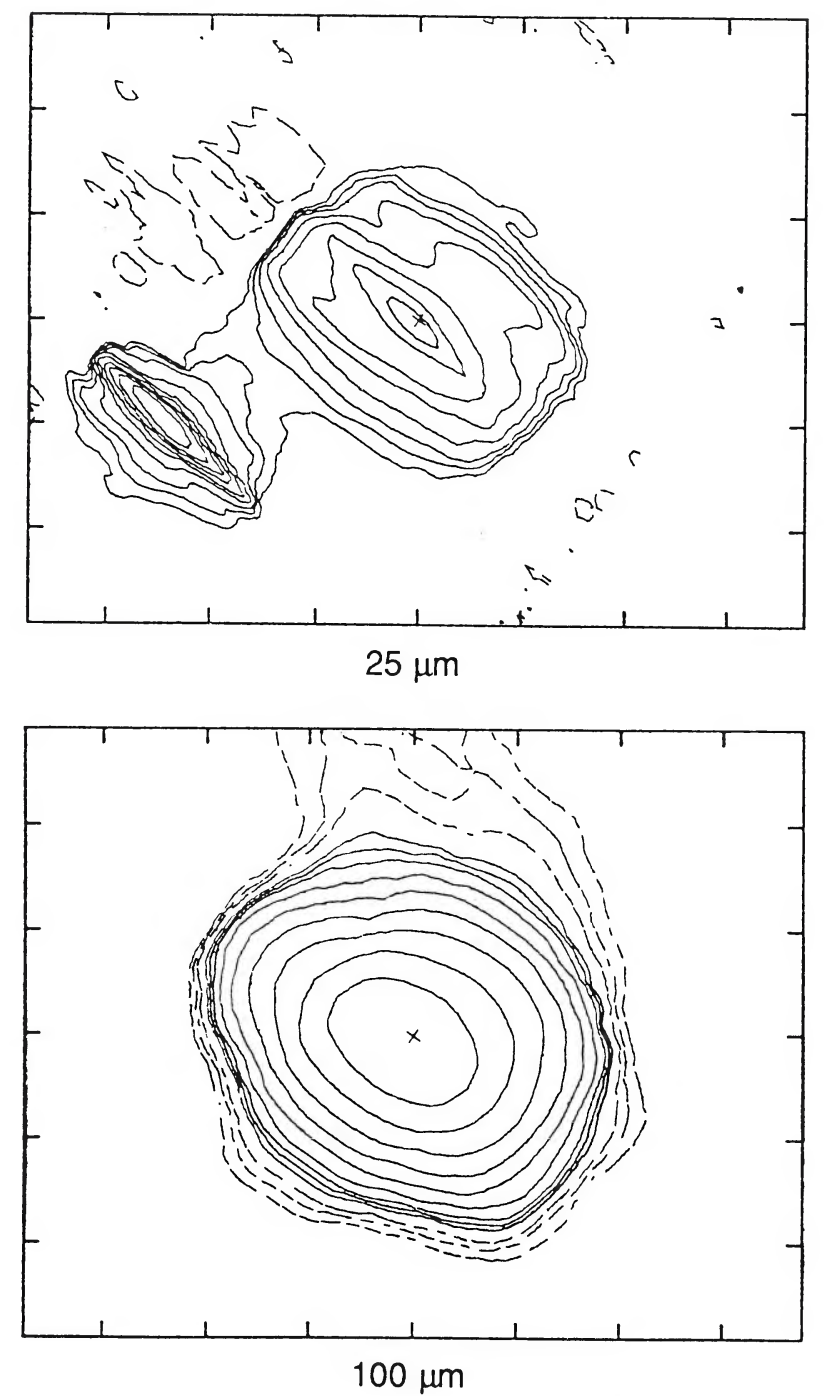

FIG. 33.-NGC 6946. (Optical photographs reproduced by permission of the California Institute of Technology. (C) 1960 National Geographic Society-Palomar Sky Survey.)

RICE et al. (see 68, 102) 

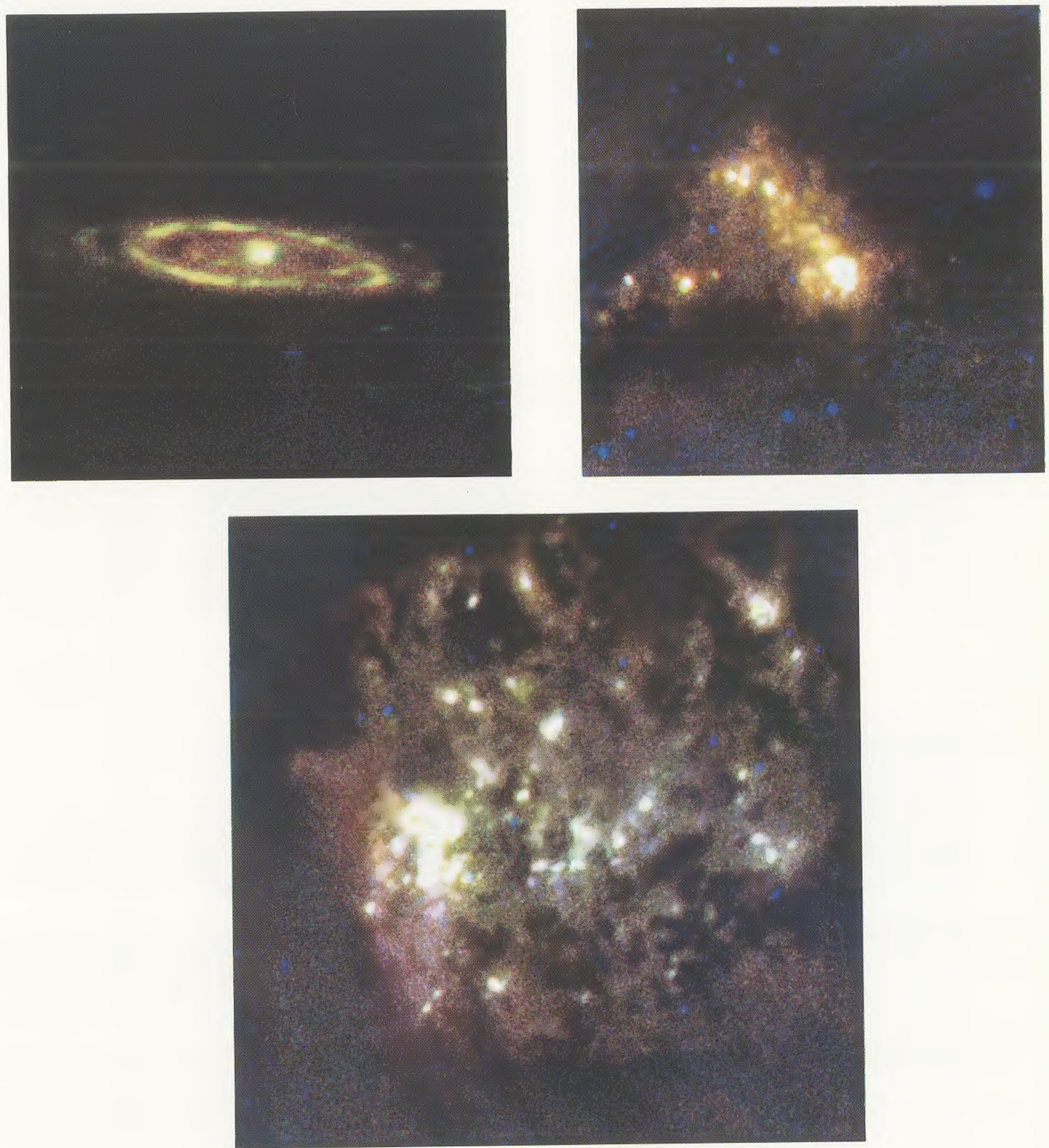

FIG. 34. - Color-coded infrared images of $(a) \mathrm{M} 31,(b)$ the SMC, and (c) the LMC, produced by combining the 12 (coded blue), 60 (coded green), and $100 \mu \mathrm{m}$ (coded red) IRAS surface brightness images of the galaxies. All maps have been smoothed to the resolution of the $100 \mu \mathrm{m}$ map.

FIG. $34 a$

FIG. $34 b$

FIG. $34 c$

Rice (e) Amperfean?)Astronomical Society - Provided by the NASA Astrophysics Data System 
TABLE 5

FAR-INFRARED $^{\mathrm{a}}$ AND OPTICAL ${ }^{\mathrm{b}}$ DIAMETERS

\begin{tabular}{|c|c|c|c|c|c|}
\hline $\begin{array}{l}\text { Name } \\
(1)\end{array}$ & $\begin{array}{c}T \\
(2)\end{array}$ & $\begin{array}{l}D_{\mathrm{IR}} \\
(3)\end{array}$ & $\underset{\text { (4) }}{D_{\mathrm{IR}} / D_{B}}$ & $\begin{array}{c}\left(A_{e} / D\right)_{\mathrm{IR}} \\
\quad(5)\end{array}$ & $\begin{array}{c}\left(A_{e} / D\right)_{B} \\
\quad(6)\end{array}$ \\
\hline N55 & $9 \mathrm{~B}$ & 26.8 & 0.83 & 0.10 & \\
\hline M31 & $3 \mathrm{~A}$ & 157.1 & 0.83 & 0.17 & 0.28 \\
\hline $\mathrm{N} 247 .$. & $7 \mathrm{X}$ & 18.3 & 0.91 & 0.18 & 0.50 \\
\hline $\mathrm{N} 253 \ldots$ & $5 \mathrm{X}$ & 26.5: & 1.06 & 0.08 : & 0.28 \\
\hline $\mathrm{N} 300 \ldots$ & $7 \mathrm{~A}$ & 18.0 & 0.89 & 0.21 & \\
\hline M33 ... & $5 \mathrm{~A}$ & 51.3 & 0.81 & 0.17 & 0.43 \\
\hline I342 $\ldots$ & $6 \mathrm{X}$ & 20.9 & 0.92 & 0.19 & 0.79 \\
\hline M81 & $2 \mathrm{~A}$ & 20.3 & 0.76 & 0.20 & 0.28 \\
\hline N3109.. & $9 \mathrm{~B}$ & 12.4 & 0.84 & & $\ldots$ \\
\hline N3628. & 3 & 14.3 & 0.96 & $<0.14$ & $\ldots$ \\
\hline N4244.. & $6 \mathrm{~A}$ & 13.0: & 0.80 & $0.24:$ & 0.28 \\
\hline N4258.. & $4 X$ & 14.1 & 0.77 & & 0.31 \\
\hline$N 4565 \ldots$ & $3 \mathrm{~A}$ & 16.2 & 0.99 & 0.14 : & 0.22 \\
\hline N4631... & $7 \mathrm{~B}$ & 16.8: & 1.11 & 0.13 : & 0.30 \\
\hline N4945 ..... & $6 \mathrm{~B}$ & 21.1: & 0.92 & $0.10:$ & \\
\hline N5055. & $4 \mathrm{~A}$ & 14.7 & 1.20 & $\ldots$ & 0.29 \\
\hline M51 ${ }^{\mathrm{c}} .$. & $4 \mathrm{~A}$ & 11.8: & 1.07 & & 0.36 \\
\hline M83 & $5 \mathrm{X}$ & 15.6: & 1.36 & 0.16 & \\
\hline M101 .. & $6 \mathrm{X}$ & 23.1 & 0.86 & 0.18 & 0.42 \\
\hline N6744..... & $4 \mathrm{X}$ & 14.0 & 0.87 & 0.22 & $\ldots$ \\
\hline N6822. & $10 \mathrm{~B}$ & 21.1: & 1.87 & 0.26 & \\
\hline N6946.. & $6 \mathrm{X}$ & 13.3: & 0.90 & 0.19 & 0.46 \\
\hline Mean ..... & $\ldots$ & $\ldots$ & 0.98 & 0.17 & 0.37 \\
\hline RMS . & $\ldots$ & $\ldots$ & 0.25 & 0.05 & 0.15 \\
\hline
\end{tabular}

${ }^{\mathrm{a}}$ Diameters measured from the $60 \mu \mathrm{m}$ map along the optical major axis to a brightness level of $25 \mathrm{mJy} \mathrm{arcmin}^{-2}$.

${ }^{b}$ Optical diameters statistically corrected for Galactic extinction, as described in RC2.

${ }^{\mathrm{c}}$ The reported diameter is twice the isophotal radius measured from the center of M51 to the southwest along the IRAS in-scan direction.

LMC were constructed from red prints provided by the Mount Wilson and Las Campanas Observatories. A second copy of the optical photograph with the $60 \mu \mathrm{m}$ map superposed is also displayed. The optical photographs and infrared maps are at the same scale; the angular scale marked on the photograph is $8^{\prime}$ for all galaxies except the Magellanic Clouds, M31 and M33. The scale of these images is noted in the figure captions.

\section{ii) Infrared Contour Maps and the IRAS Beam}

The contour levels of the infrared images are scaled by the map $1 \sigma$ mean noise level. The noise level of each wavelength map in units of mJy arcmin ${ }^{-2}$ is listed in Table 6. Unless otherwise noted in the figure caption, the dashed contours are at 3,6 , and $9 \sigma$ below the map background level, and the solid contours are at 3,6 , and $10 \sigma$, with brighter levels scaled logarithmically by 2 from $10 \sigma$ (i.e., 20, 40, $80 \sigma$, etc.). The faintest contours of the maps should be viewed with caution given the emission from foreground Galactic "cirrus" and the possibility of inadequate background emission removal.

The optical center of the galaxy, as given in Table 1, is marked on each infrared map. For all but the largest galaxies, the tick marks on the maps are spaced by $4^{\prime}$. For the galaxies with maps at a different scale (NGC 55, M31, NGC 253,
TABLE 6

Galaxies Displayed in the Atlas

\begin{tabular}{|c|c|c|c|c|c|}
\hline \multirow[b]{2}{*}{ FigurE } & \multirow[b]{2}{*}{ NAME } & \multicolumn{4}{|c|}{ MAP NoISE LeVEL $\left(\mathrm{mJy} \operatorname{arcmin}^{-2}\right)$} \\
\hline & & $12 \mu \mathrm{m}$ & $25 \mu \mathrm{m}$ & $60 \mu \mathrm{m}$ & $100 \mu \mathrm{m}$ \\
\hline 2 & N55 & 3.98 & 5.26 & 3.77 & 7.82 \\
\hline 3 & M31 & 5.74 & 7.32 & 4.52 & 8.44 \\
\hline 4. & $\mathrm{~N} 247$ & 11.71 & 15.88 & 8.73 & 11.28 \\
\hline 5 . & $\mathrm{N} 253$ & 11.59 & 14.59 & 8.70 & 11.21 \\
\hline 6 . & SMC & 4.74 & 4.84 & 3.91 & 6.06 \\
\hline 7. & N300 & 11.20 & 13.57 & 9.38 & 13.09 \\
\hline 8 . & M33 & 6.61 & 10.95 & 4.68 & 9.82 \\
\hline 9 & N1313 & 5.15 & 6.07 & 4.94 & 9.86 \\
\hline 10 & I 342 & 4.91 & 5.85 & 4.27 & 11.30 \\
\hline 11 & N1448 & 3.52 & 3.97 & 2.99 & 5.73 \\
\hline 12 & LMC & 5.81 & 5.97 & 7.21 & 16.39 \\
\hline 13. & N2403 & 5.19 & 7.45 & 3.93 & 7.56 \\
\hline 14. & M81 & 4.56 & 5.86 & 3.84 & 7.30 \\
\hline 15 & N3109 & 4.03 & 5.42 & 3.07 & 5.72 \\
\hline 16 & I 2574 & 4.96 & 6.05 & 4.41 & 7.51 \\
\hline 17. & N3628 & 12.40 & 20.55 & 8.83 & 11.91 \\
\hline 18 . & N4236 & 4.36 & 5.35 & 3.62 & 5.93 \\
\hline 19. & N4244 & 4.71 & 6.82 & 3.61 & 5.95 \\
\hline 20 . & N4258 & 4.51 & 5.97 & 3.51 & 6.40 \\
\hline 21 & N4395 & 11.27 & 13.96 & 8.35 & 10.98 \\
\hline 22 & N4565 & 5.01 & 6.88 & 3.93 & 7.06 \\
\hline 23 & N4631 & 4.65 & 6.52 & 3.53 & 5.70 \\
\hline 24 & N4656 & 4.72 & 6.46 & 3.57 & 5.28 \\
\hline 25 . & N4725 & 11.36 & 15.05 & 8.15 & 9.82 \\
\hline 26 & N4945 & 5.73 & 6.78 & 5.62 & 12.30 \\
\hline 27 & N5055 & 4.51 & 5.51 & 3.59 & 6.71 \\
\hline 28 . & M51 & 5.07 & 6.30 & 3.93 & 6.61 \\
\hline 29 . & M83 & 8.63 & 14.84 & 7.58 & 18.96 \\
\hline $30 \ldots \ldots \ldots$ & M101 & 1.26 & 1.49 & 1.16 & 1.86 \\
\hline $31 \ldots$ & N6744 & 3.99 & 4.57 & 4.36 & 11.56 \\
\hline 32 & N6822 & 3.24 & 6.94 & 2.58 & 4.13 \\
\hline 33 . & N6946 & 3.61 & 3.95 & 3.13 & 8.06 \\
\hline
\end{tabular}

SMC, M33, LMC, NGC 4945, M101, and NGC 6822), the tick mark spacing is noted in the figure caption.

Note that even in those maps showing structural features, the "resolved" features may reflect both the noncircular, undeconvolved IRAS beam shape and the various scanning directions of the individual pointed observation or survey scan maps co-added to produce the maps shown. The beam shape, represented by the $\frac{1}{4}, \frac{1}{2}$, and $\frac{3}{4}$ power brightness level contours of an IRAS point source, is displayed on the maps of NGC 55 (Fig. 2). The beam has been oriented along the scanning direction of the observations of the galaxy.

\section{ANALYSIS AND DISCUSSION}

A preliminary analysis of the catalog and a discussion of the atlas maps are presented below. The detailed discussion of these data, including the radial distribution of the infrared surface brightness and colors, arm/disk and nuclear/disk contrasts, comparisons with optical, $\mathrm{H} \mathrm{I}$ and $\mathrm{CO}$ images and profiles, and an intercomparison among the atlas galaxies of all these parameters, is deferred to Paper II. 


\section{a) Detection Rates}

The distribution by optical morphological type of the cata$\log$ galaxies and of their IRAS detection rates are tabulated in Table 7. The six galaxies not detected are all ellipticals, and the two ellipticals detected, NGC 185 and NGC 205, are well known to have low levels of star formation (Hodge 1963, 1973; Gallagher and Mould 1981). Seventy-five percent (59 of 79) of the detected galaxies were observed at all four IRAS wavelengths, and all but three galaxies - the S0 galaxies NGC 1023, NGC 3115, and NGC 4762, which were seen only at 12 $\mu \mathrm{m}$-were detected at both 60 and $100 \mu \mathrm{m}$.

\section{b) Far-Infrared and Optical Sizes}

The mean ratio of the $60 \mu \mathrm{m}$ to blue-light isophotal diameters, reported in Table 5, is $0.98 \pm 0.25$. The most discrepant ratio is for NGC 6822. NGC 6822, a member of the Local Group, is a low surface brightness dwarf irregular system at low Galactic latitude $(b=-18: 4)$. These two factors, and possibly confusion due to "cirrus," may account for the discrepant optical and infrared sizes of this galaxy.

For the 11 galaxies for which both infrared and optical effective aperture diameter measurements are available, the mean radial distribution of the infrared emission, $\left\langle\left(A_{e} / D\right)_{\mathrm{IR}}\right\rangle=0.17 \pm 0.05$, is more centrally concentrated than that of the blue-light emission $\left\langle\left(A_{e} / D\right)_{B}\right\rangle=0.37 \pm 0.15$. While a discussion of this result is deferred to Paper II, one factor likely to contribute to the larger ratio of the blue-light distribution is the extinction of the blue light toward the central regions of the galaxies.

While galaxies with infrared extents that significantly exceed their optical sizes may exist, the overall agreement between the optical and far-infrared sizes of the galaxies best resolved by IRAS presented in this catalog suggests that the RC2 "large optical galaxy" sample, along with the secondary sample presented in the Appendix, comprise a complete set of galaxies likely to have $60 \mu \mathrm{m}$ extents greater than $8^{\prime}$ (and for which, because of source extent, the data reduction techniques of this study are needed to obtain "total" IRAS flux densities).

\section{c) General Far-Infrared Morphology}

Although the IRAS spatial resolution decreases with increasing wavelength, the infrared images and profiles presented in the atlas are typically most interesting at $60 \mu \mathrm{m}$. Galaxies emit substantially more flux at the longer IRAS wavelengths, and, in some cases the large galaxies studied here do not emit strongly enough in the outer regions to be detected as extended sources at 12 and $25 \mu \mathrm{m}$.

We have attempted to "classify" the $60 \mu \mathrm{m}$ images and brightness profiles of the galaxies displayed in the atlas according to a simple scheme that parallels the Hubble bulgeto-disk classification scheme for optical morphology. The basic criterion used here was the shape of the $60 \mu \mathrm{m}$ in-scan radial profile, in particular, the degree of central concentration of the $60 \mu \mathrm{m}$ emission in relation to the disk emission. (Note that in this far-infrared "bulge-to-disk" reclassification scheme, the $60 \mu \mathrm{m}$ bulge emission does not correspond to the galaxy stellar nuclear bulge.) This classification scheme is limited by the varying linear scale sizes resolvable in the galaxies and the orientation of the IRAS scanning direction with respect to the galaxy position and inclination angles. Therefore, we have separated the 25 highest resolution images into four bins only, and refer to them as "groups" rather than as well-defined classes. A fifth group contains highly inclined galaxies which were scanned in a direction close to the minor axis, and for which there is insufficient resolution to place them in one of the other four groups.

This grouping scheme is based only on the $60 \mu \mathrm{m}$ images and profiles and not on the data at the other wavelengths.

Group 1: IC 342, NGC 4656, NGC 4945, NGC 5236 (M83), $N G C$ 6946. - These five galaxies show the largest contrast of the $60 \mu \mathrm{m}$ "bulge-to-disk" emission among the atlas galaxies

TABLE 7

Morphological Distribution and Detection Rates

\begin{tabular}{|c|c|c|c|c|c|c|c|c|}
\hline \multirow[b]{2}{*}{ SAMPLE } & \multicolumn{8}{|c|}{ TYPE } \\
\hline & $\mathrm{E}$ & So & S0/a-Sab & $\mathrm{Sb}-\mathrm{Sbc}$ & $\mathrm{Sc}-\mathrm{Scd}$ & Sd-Im & I0 & Tota \\
\hline Optical. & 8 & 5 & 12 & 17 & 22 & 20 & 1 & 85 \\
\hline $12 \mu \mathrm{m}$ detection & 2 & 4 & 11 & 16 & 21 & 12 & 1 & 67 \\
\hline $25 \mu \mathrm{m}$ detection & 1 & 1 & 10 & 16 & 18 & 15 & 1 & 62 \\
\hline $60 \mu \mathrm{m}$ detection...$\ldots$. & 2 & 2 & 12 & 17 & 22 & 20 & 1 & 76 \\
\hline $100 \mu \mathrm{m}$ detection & 2 & 2 & 12 & 17 & 22 & 20 & 1 & 76 \\
\hline 4 band detection ........ & 1 & 1 & 10 & 16 & 18 & 12 & 1 & 59 \\
\hline No detection ........... & 6 & 0 & 0 & 0 & 0 & 0 & 0 & 6 \\
\hline
\end{tabular}

FIG. 1.-Profile cuts along the IRAS scanning direction of the $12,25,60$, and $100 \mu \mathrm{m}$ surface brightness images of the "large optical galaxies." Wavelength profile plots of all galaxies detected brighter than $6 \sigma$ are presented (the SMC and the LMC are excluded). The cuts are centered on the galaxy optical center and are scaled by the maximum intensity found along the cut. For each galaxy the position angle of the in-scan cuts is indicated. The lighter curve marked with small circles is the in-scan profile of IRC +10011 , a representative IRAS point source. An offset has been applied to redefine the profile zero level of the images for which Galactic "cirrus" resulted in poor background removal. 
0
$\cdots$
$\vdots$
$\vdots$
0
0
$\vdots$
0
1
0
0
0
$\infty$
$\infty$
0
7

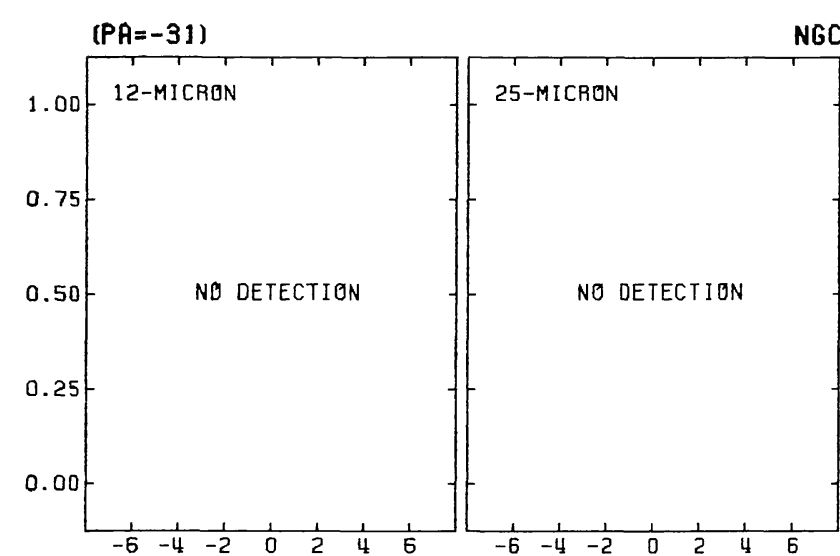

NGC 45
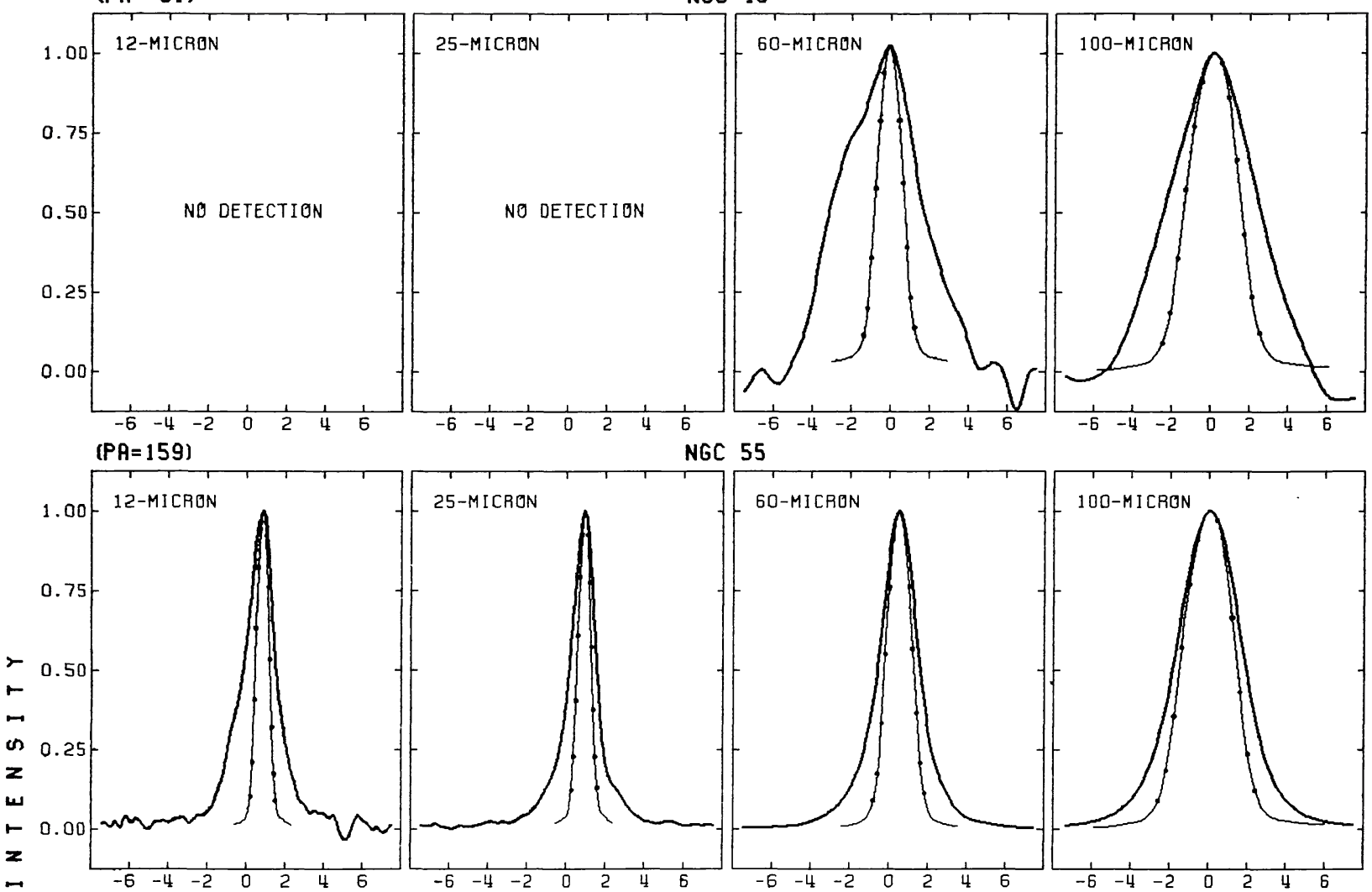

GC 55
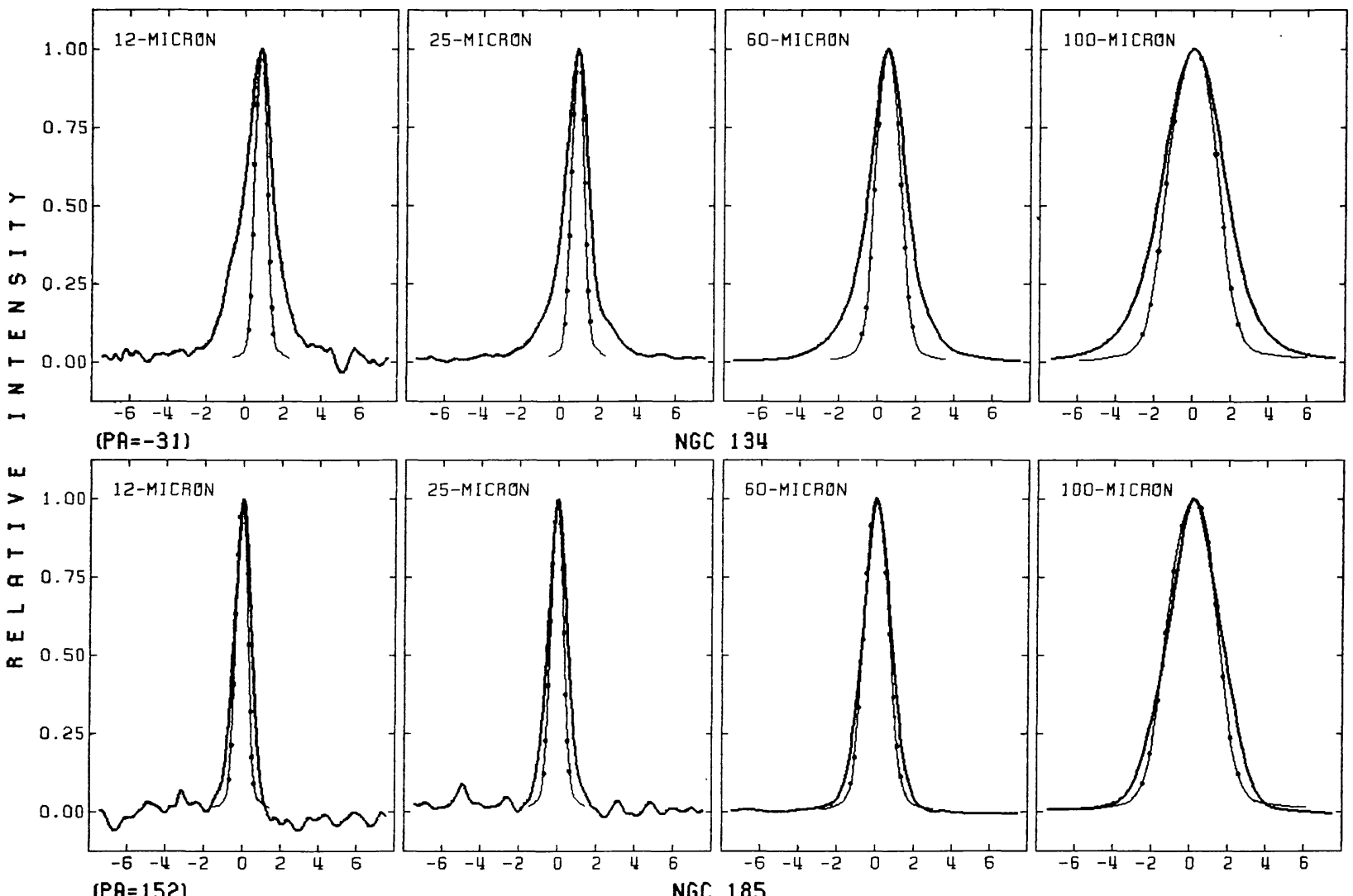

NGC 134
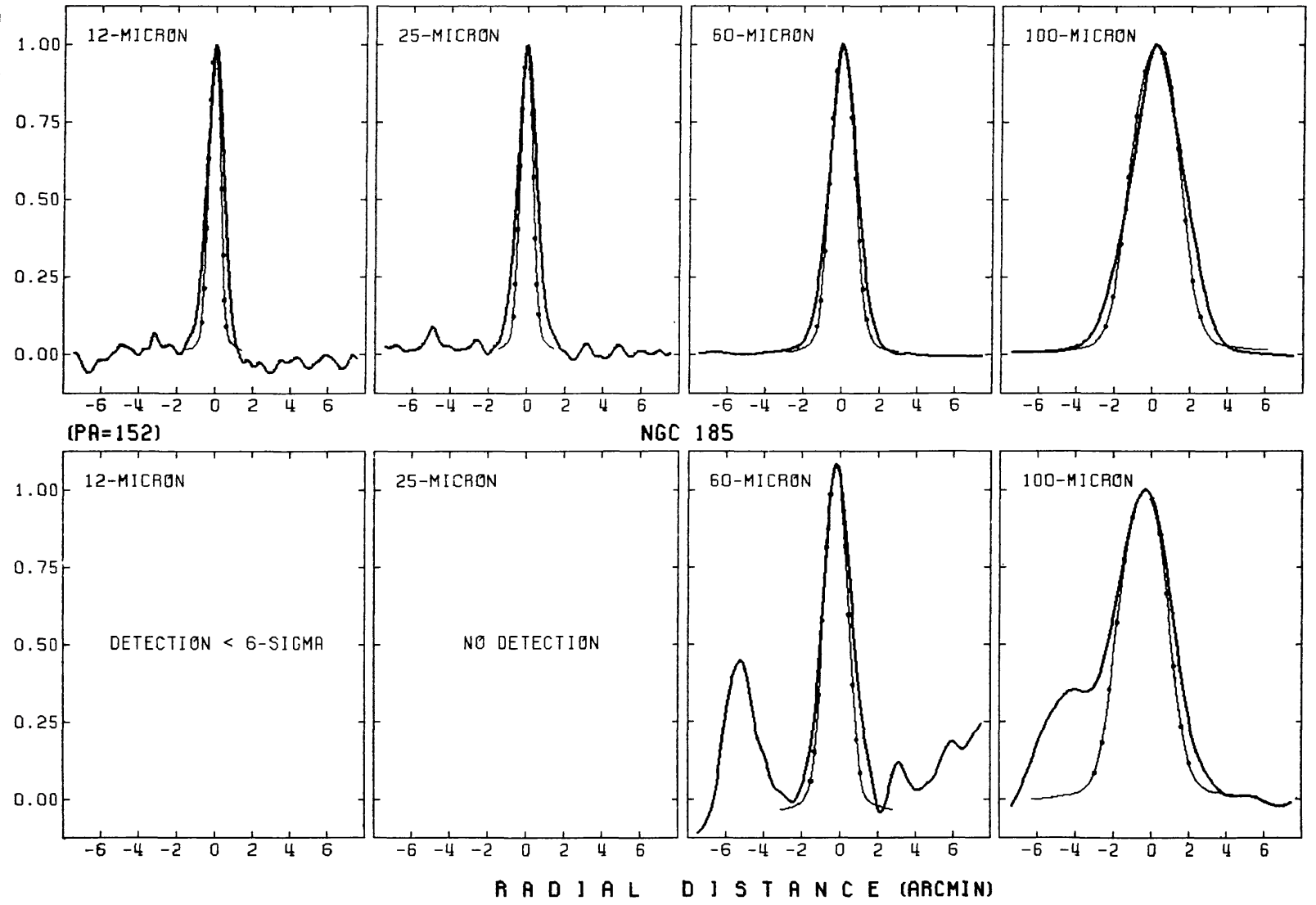


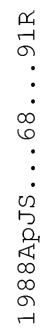
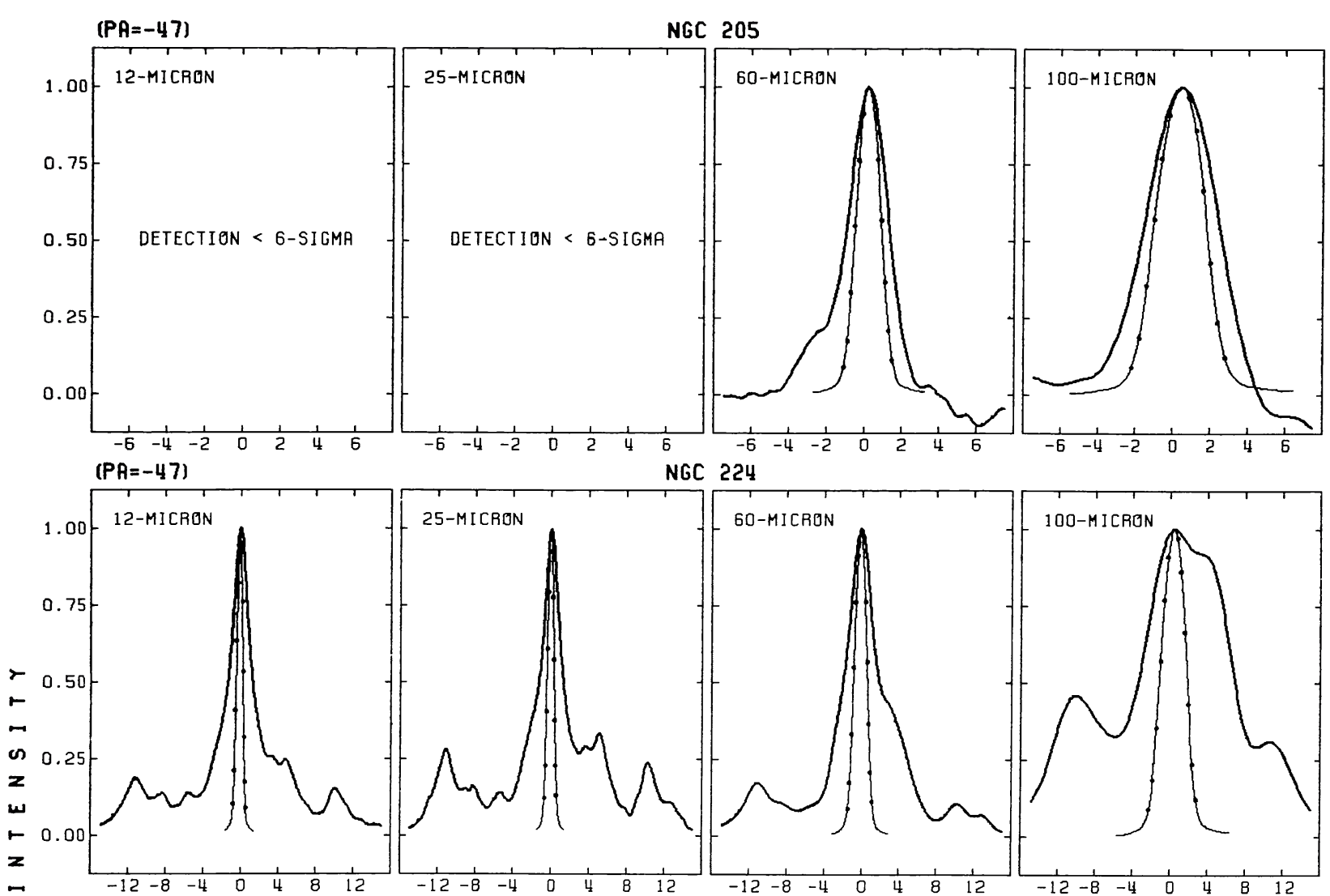

NGC 224

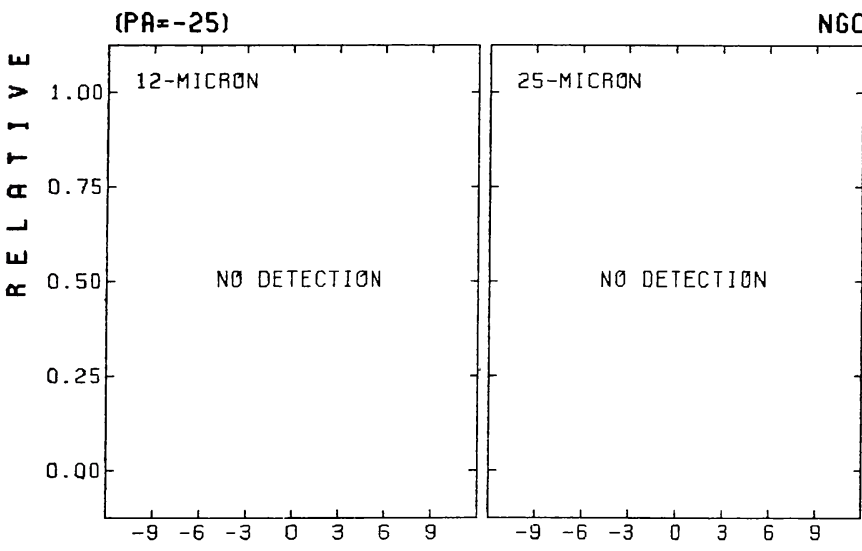

NGC 247

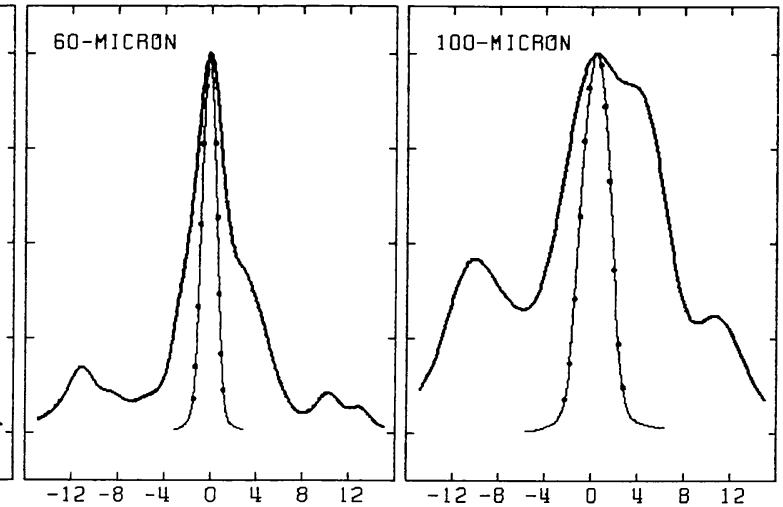

$(P A=141)$

NGC 253
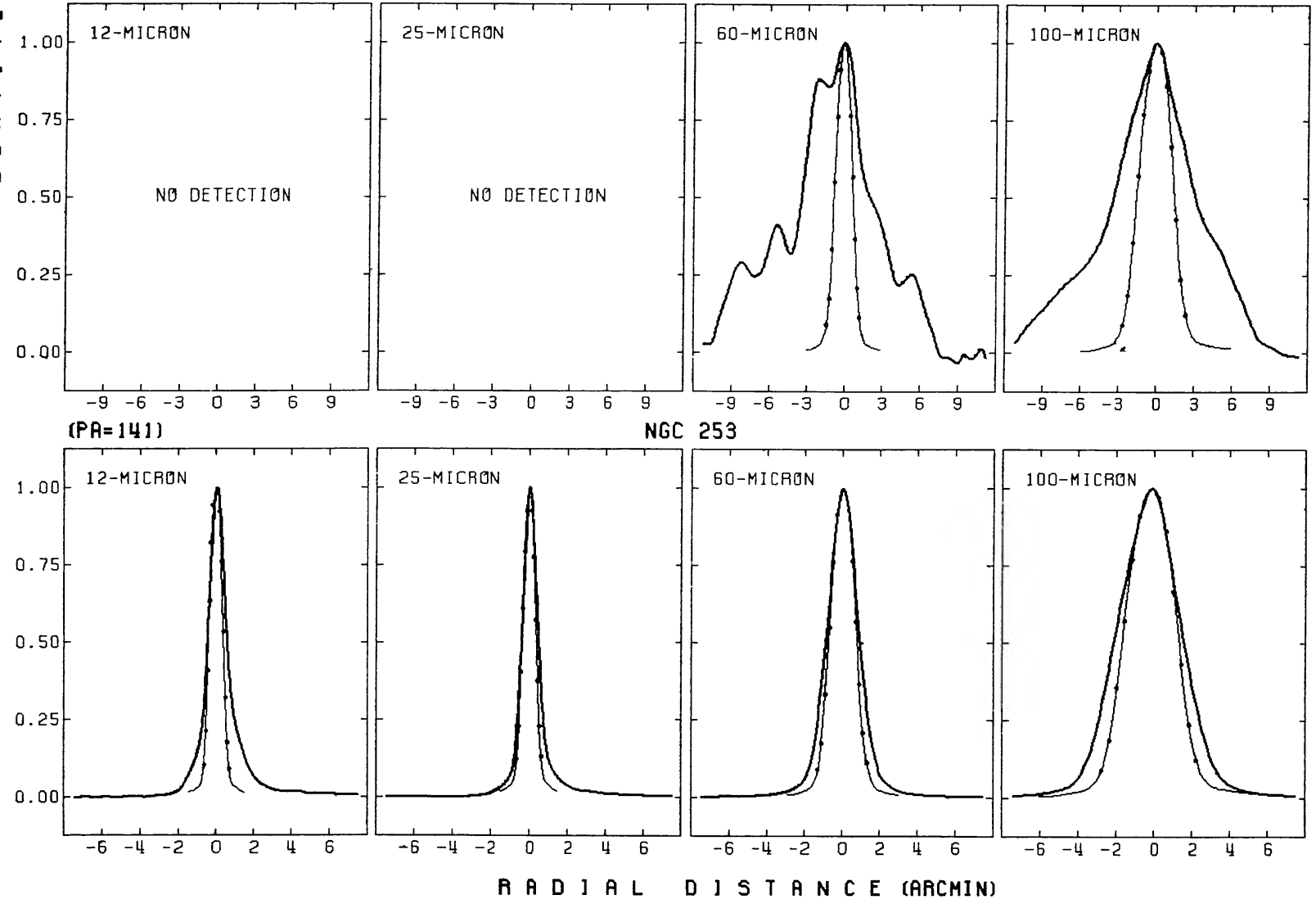

FIG. 1-Continued 


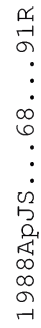
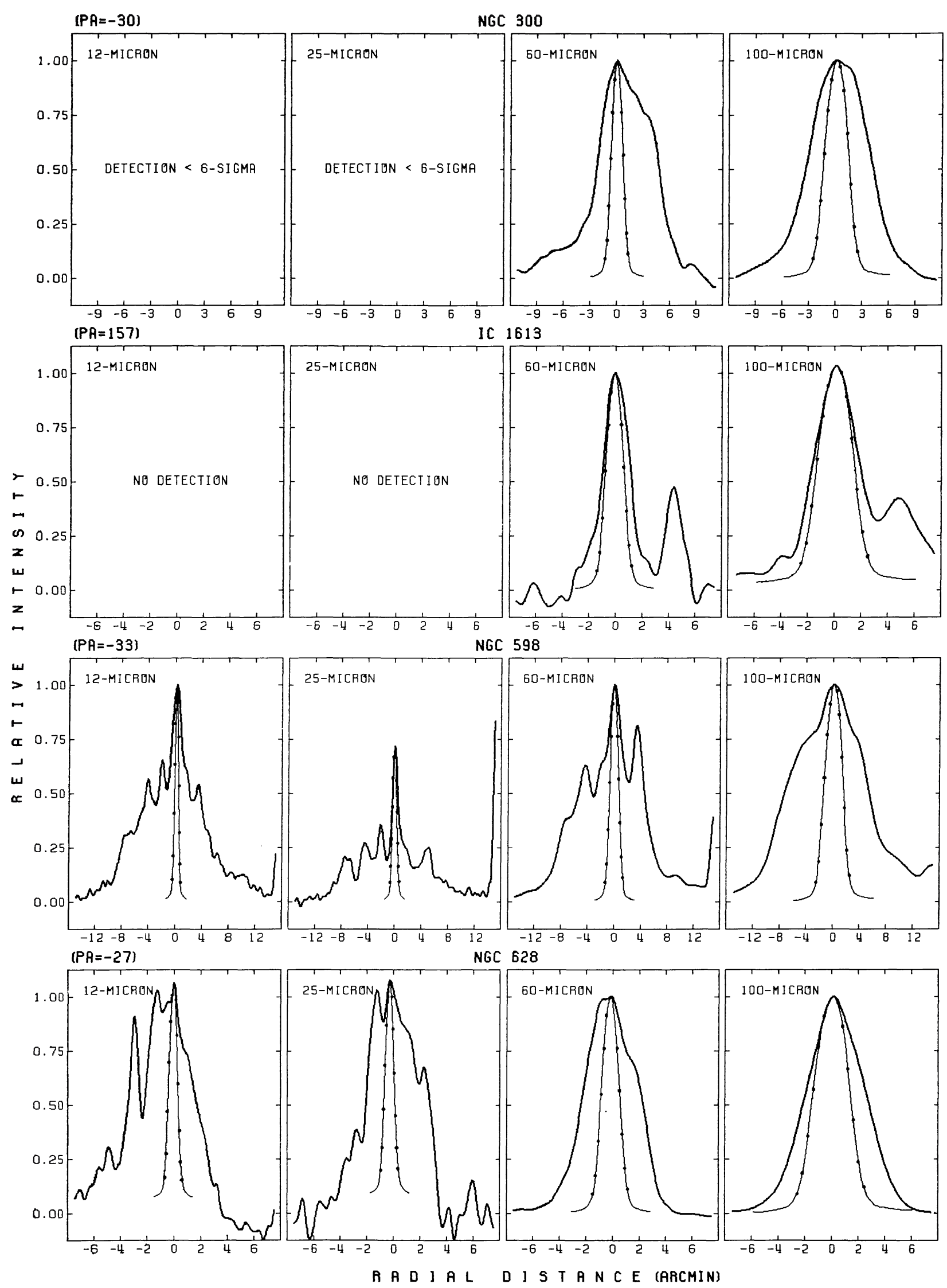

FIG. 1-Continued 


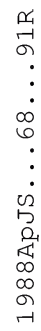

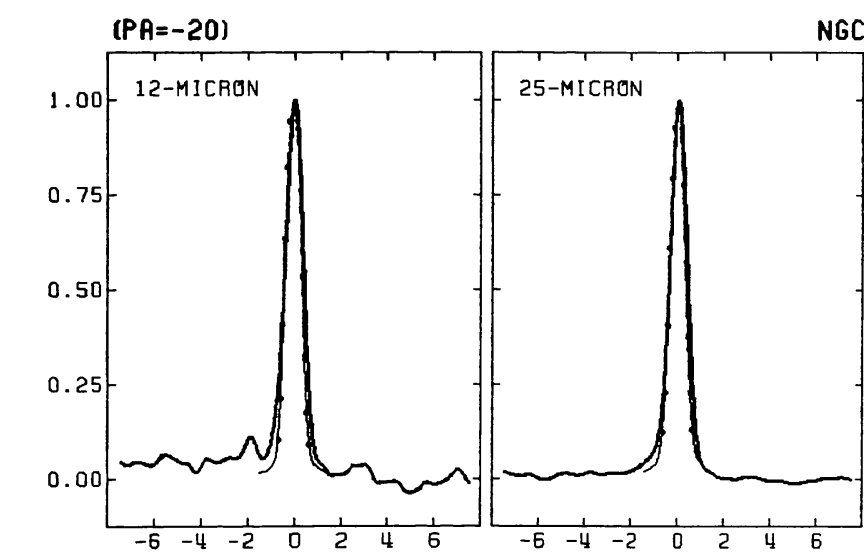

NGC 660
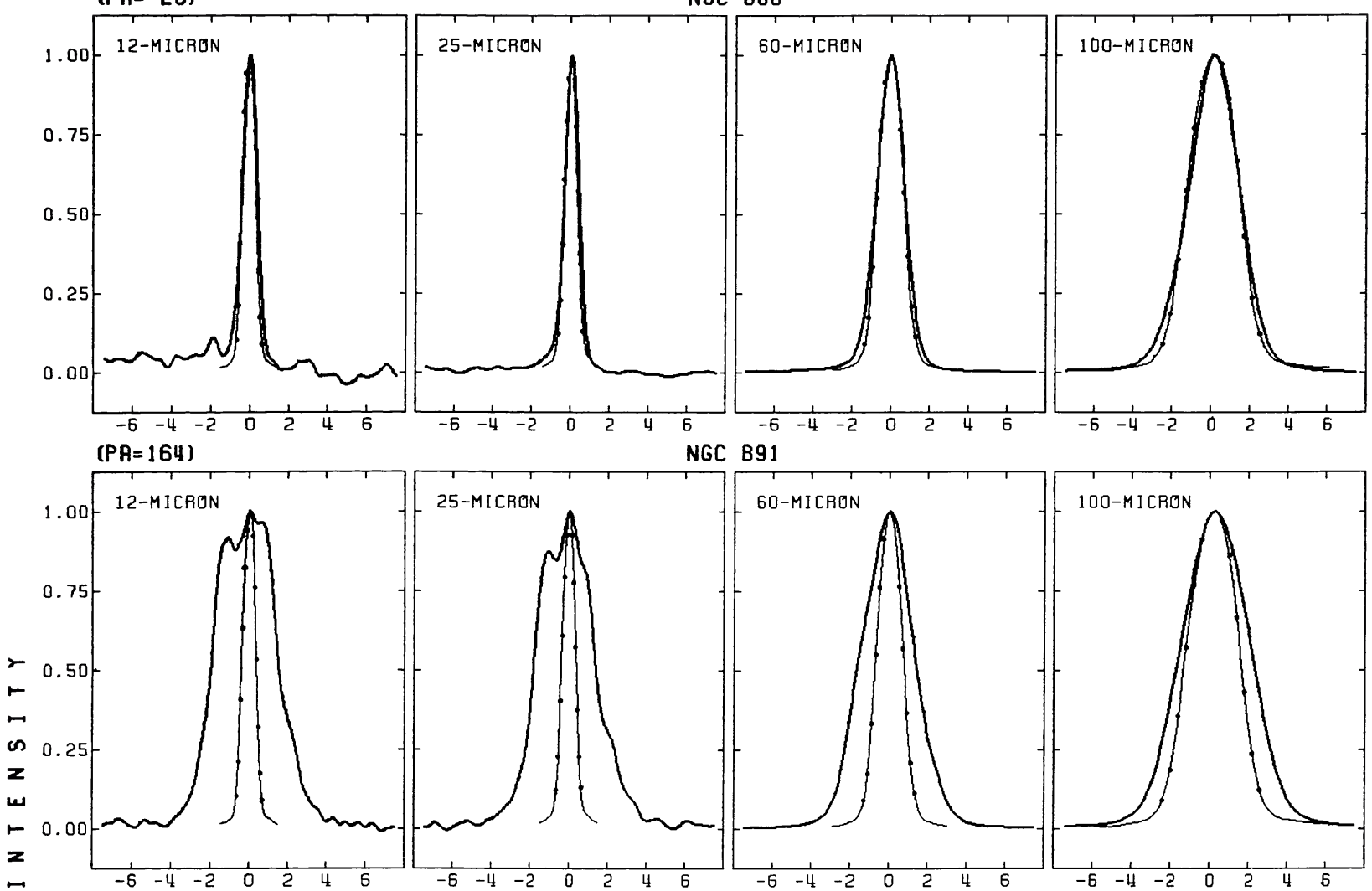

\section{1}
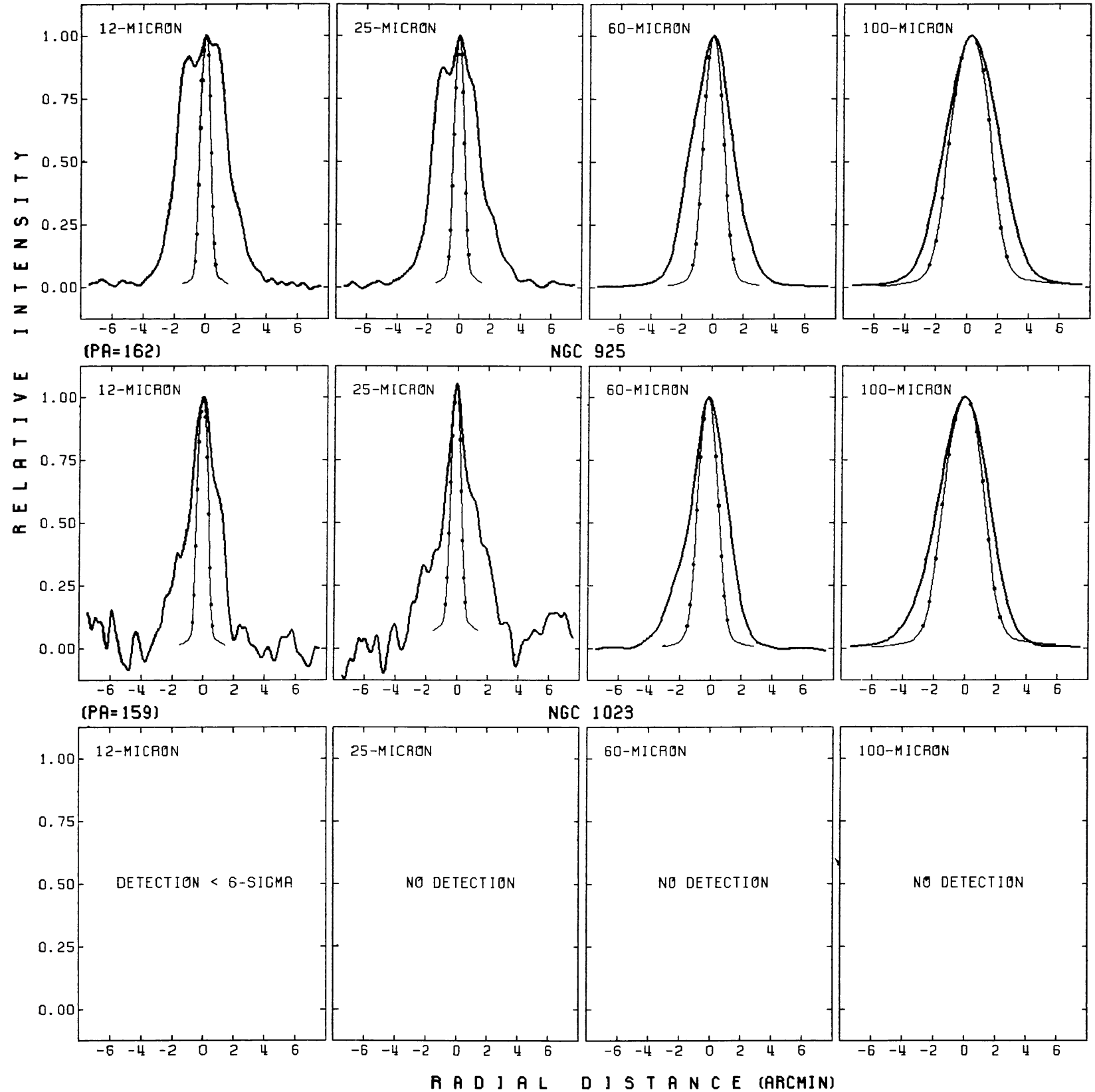

FIg. 1-Continued 
0
$\cdots$
$\vdots$
$\vdots$
0
0
$\vdots$
0
1
0
0
0
$\infty$
$\infty$
0
7
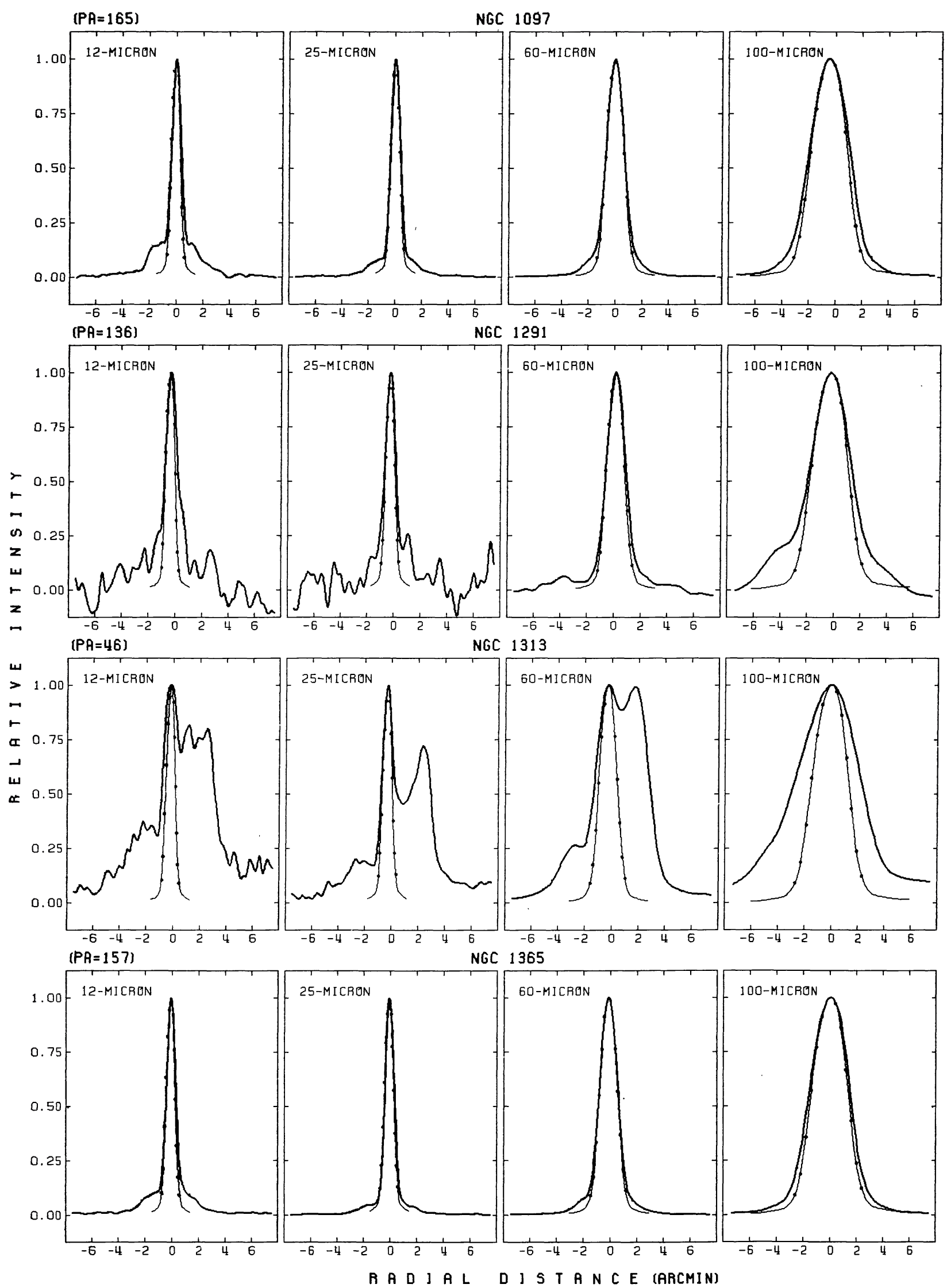

FIg. 1-Continued 
0
$\cdots$
$\vdots$
$\vdots$
0
0
$\vdots$
0
1
0
0
0
$\infty$
$\infty$
0
7
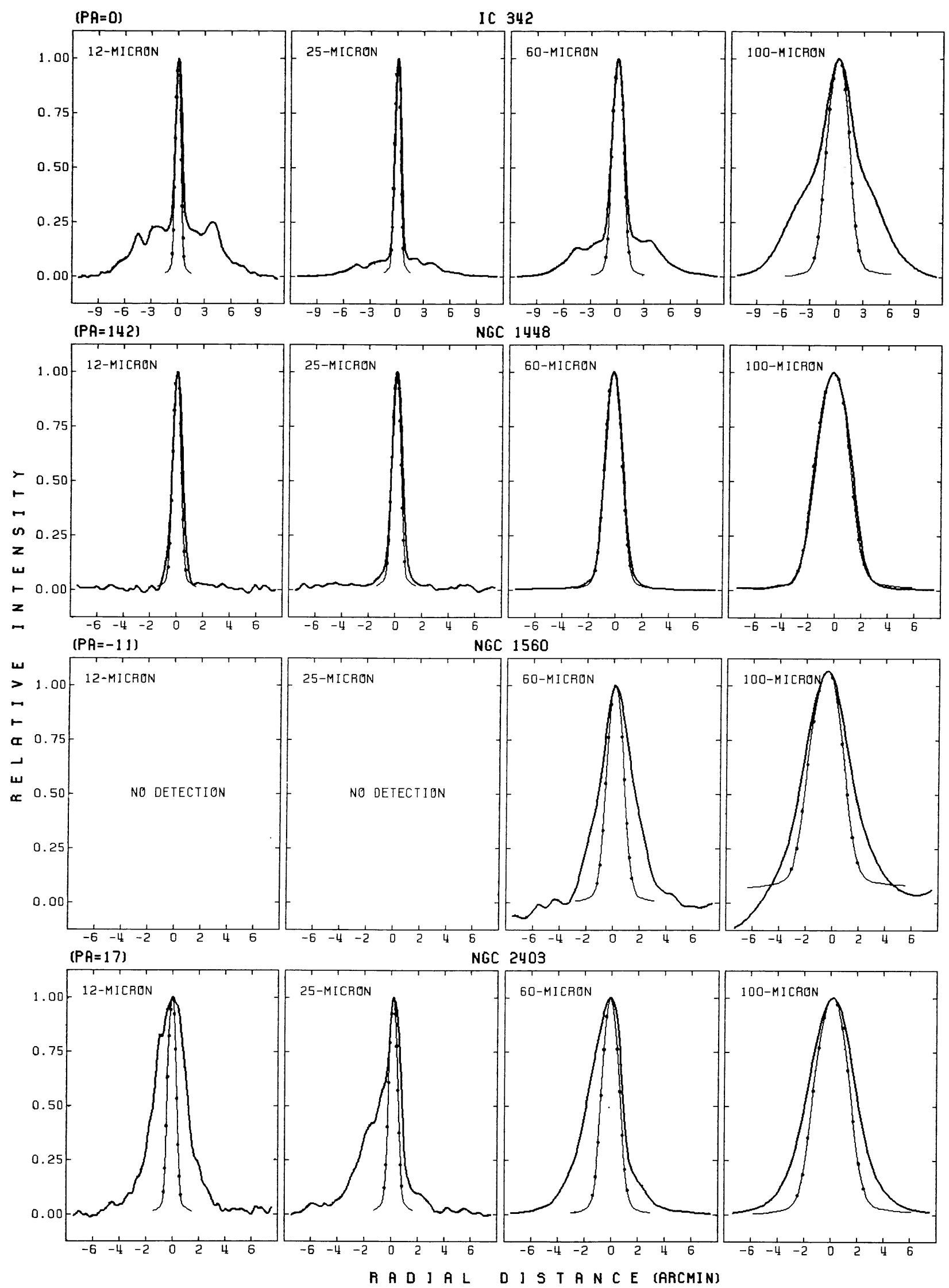

FIG. 1-Continued 


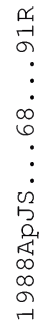
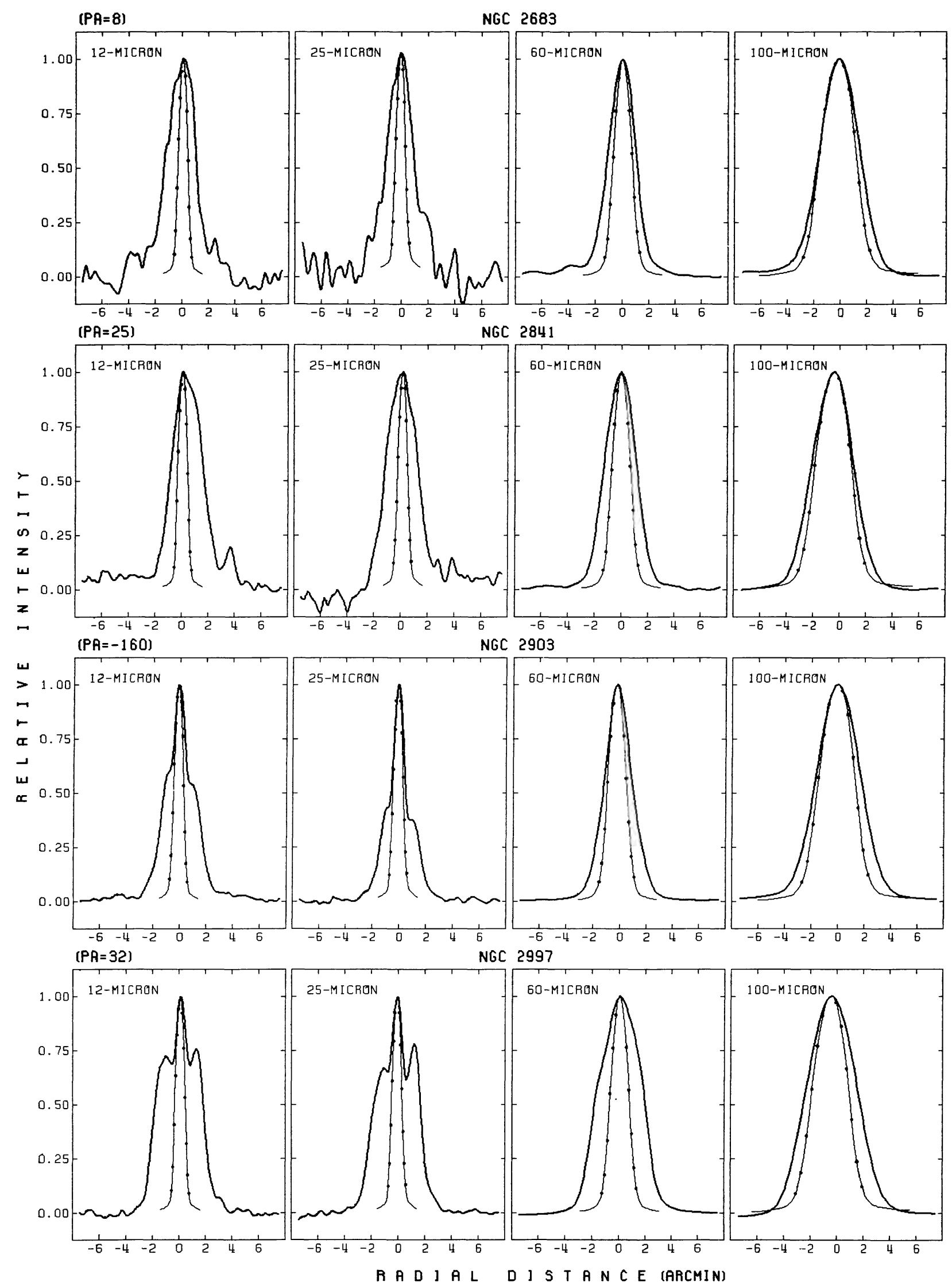

FIG. 1-Continued 
0
$\cdots$
$\vdots$
$\vdots$
0
0
$\vdots$
$\vdots$
0
0
0
4
$\infty$
$\infty$
0
0
7
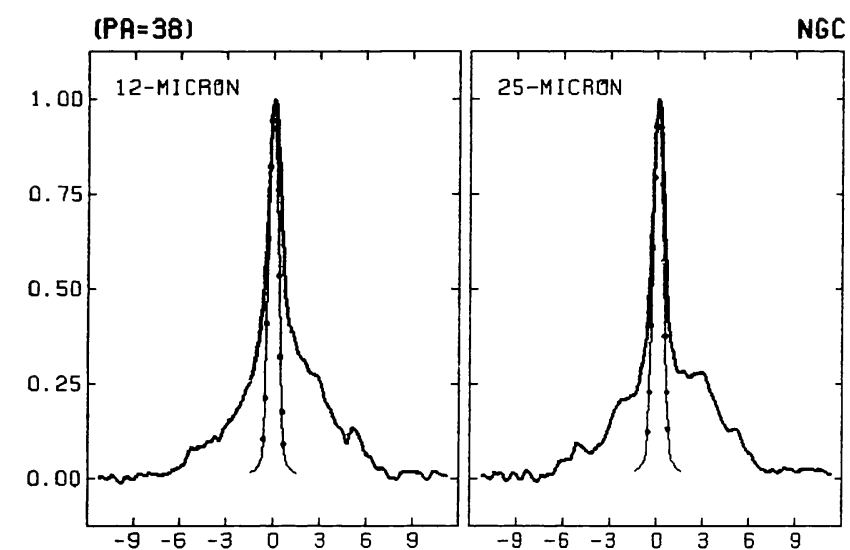

NGC 3031
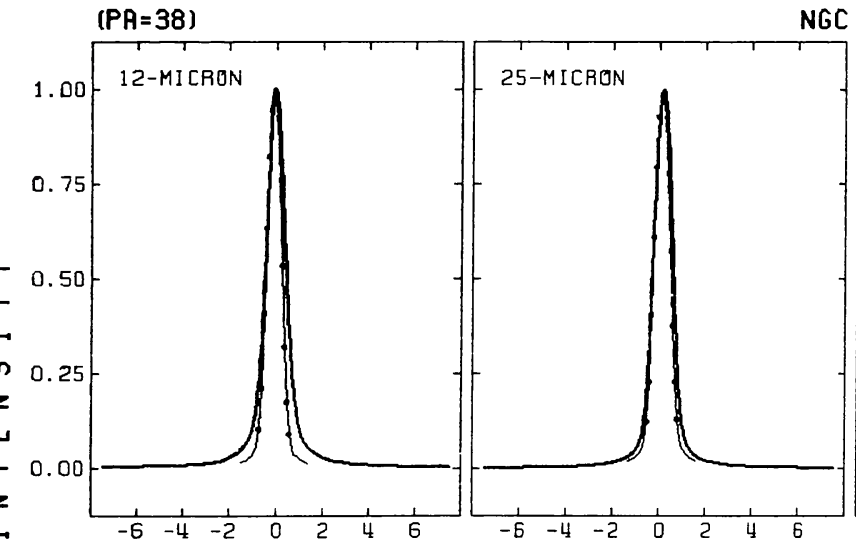

NGC 3034
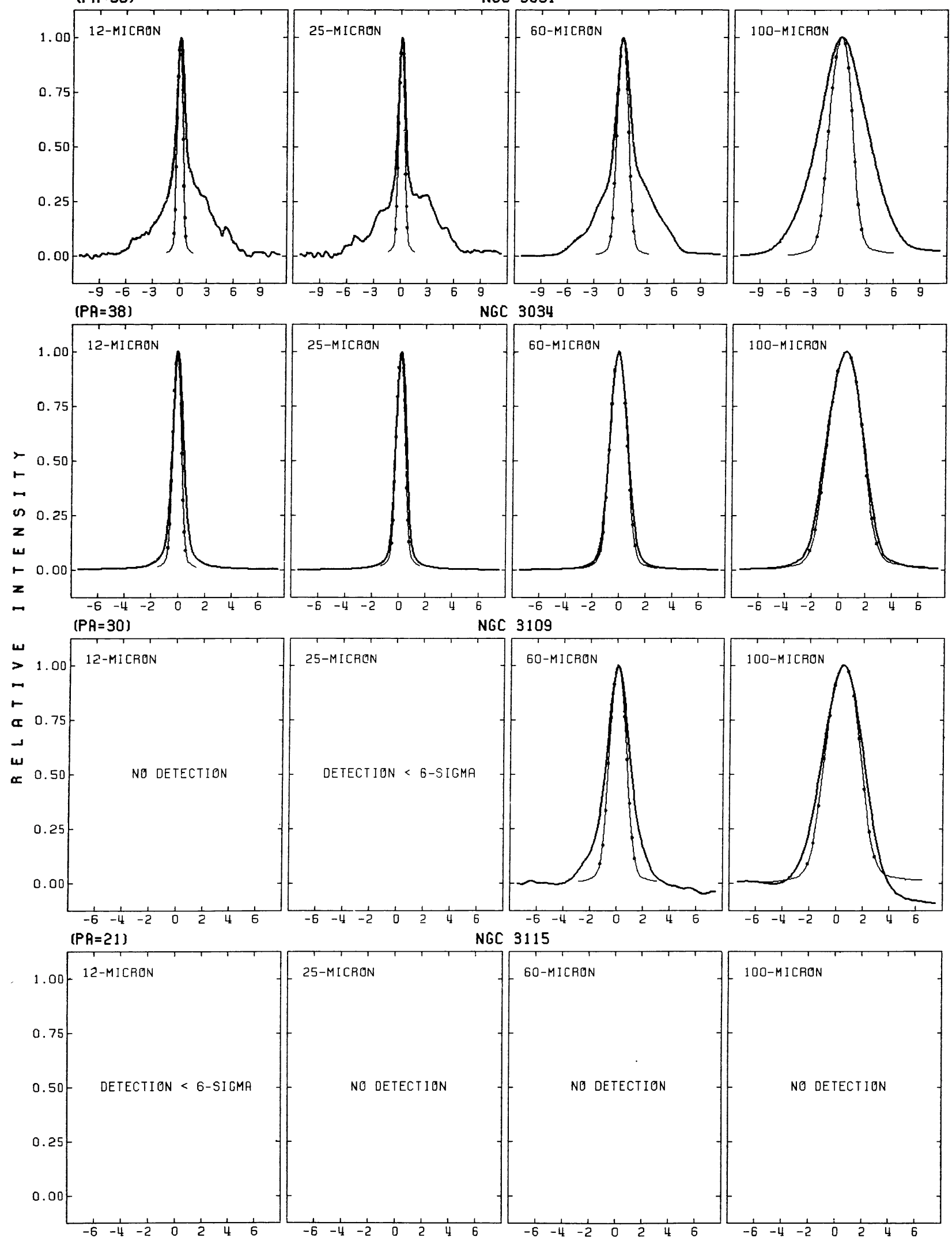

NGC 3115

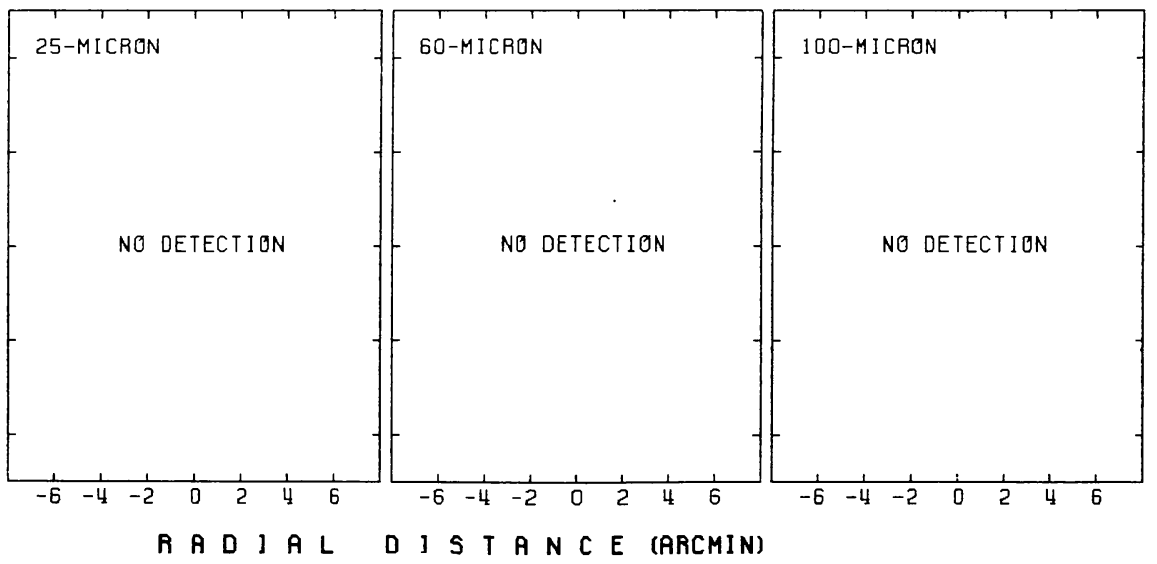

FIG. 1-Continued 
0
$\cdots$
$\vdots$
$\vdots$
0
0
$\vdots$
0
1
0
0
0
$\infty$
$\infty$
0
7

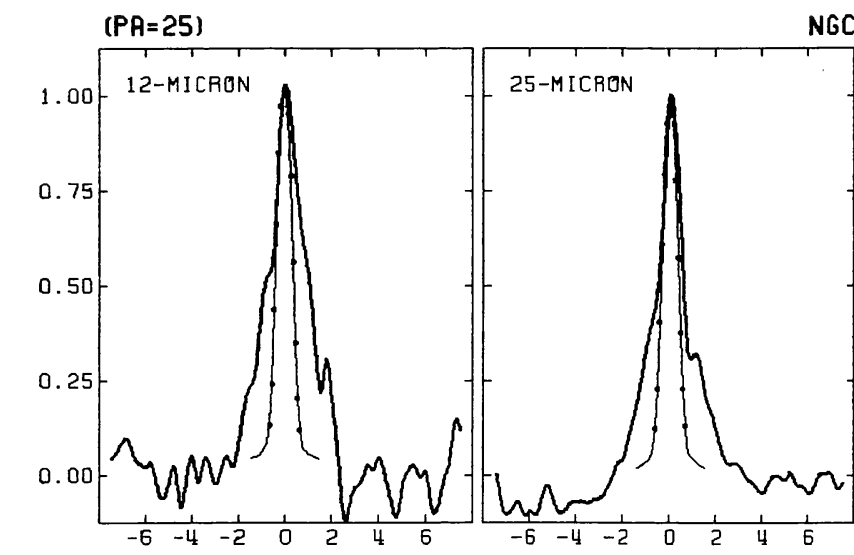

NGC 3198
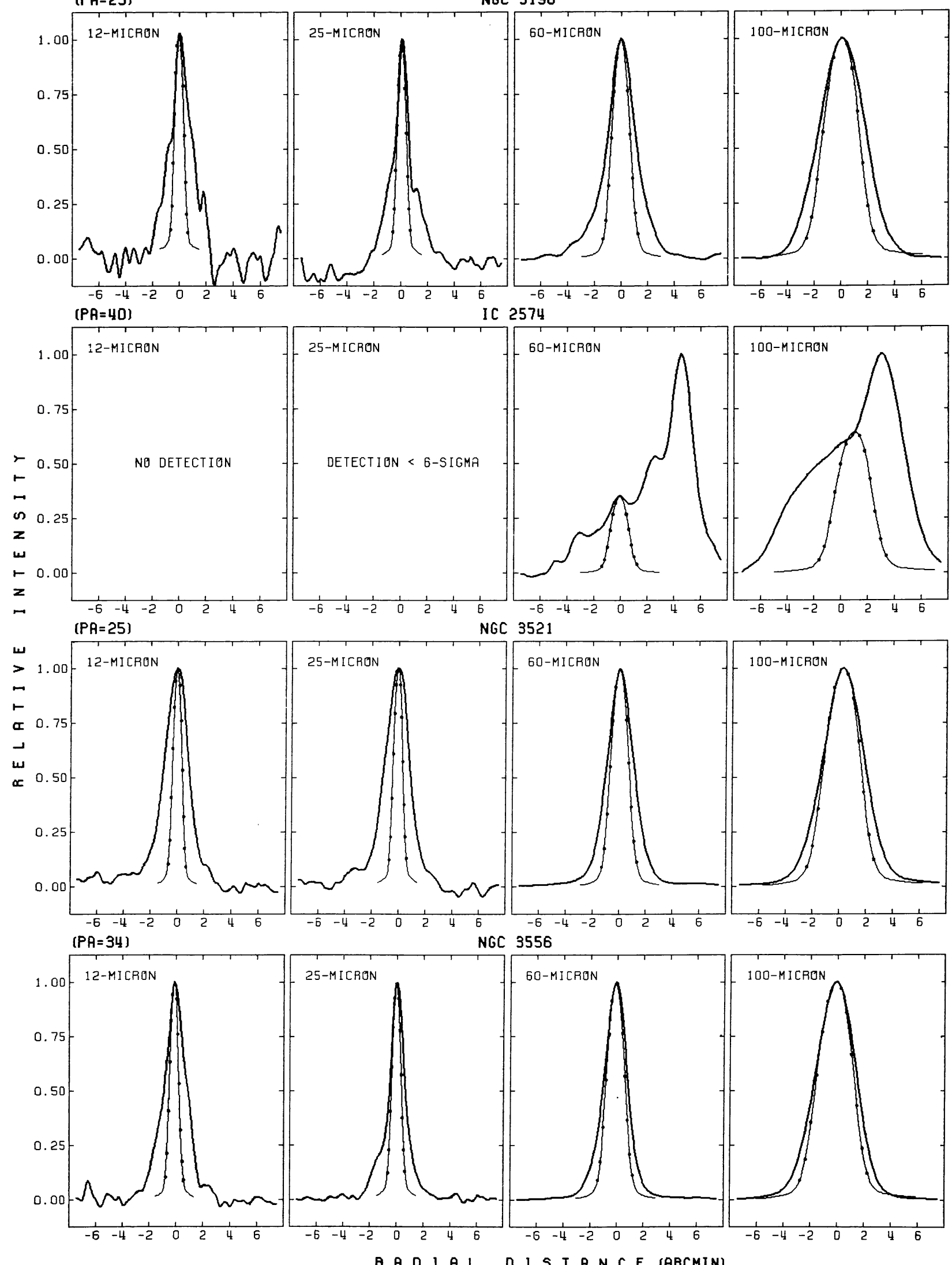

FIG. 1-Continued 


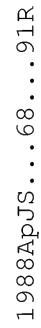
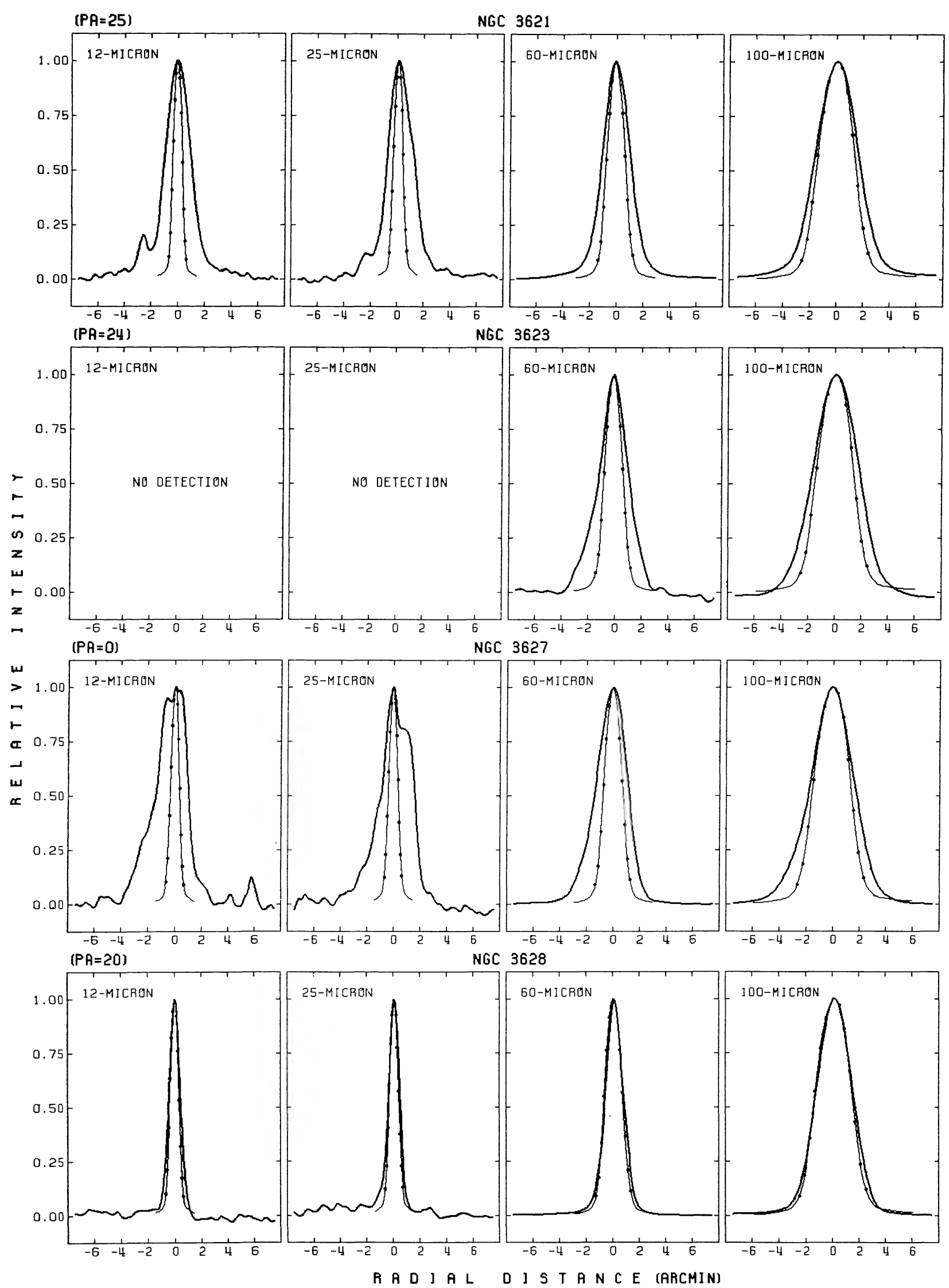

FIG. 1-Continued

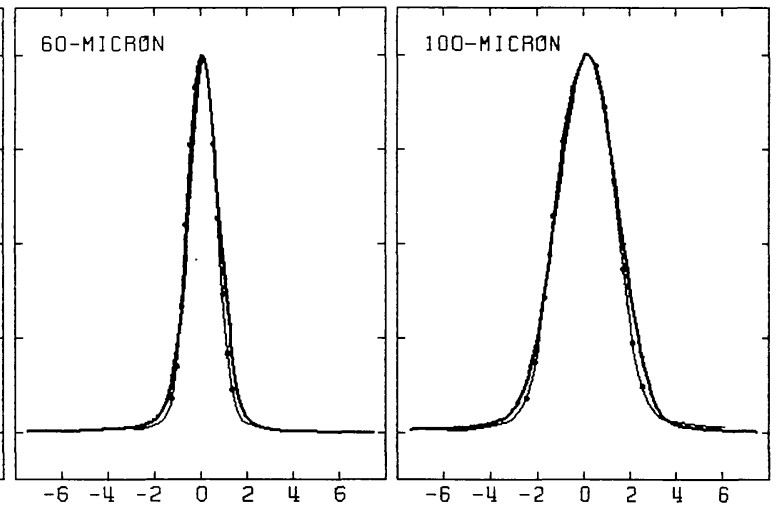

114 
0
$\cdots$
$\vdots$
$\vdots$
0
0
$\vdots$
0
1
0
0
0
$\infty$
$\infty$
0
7
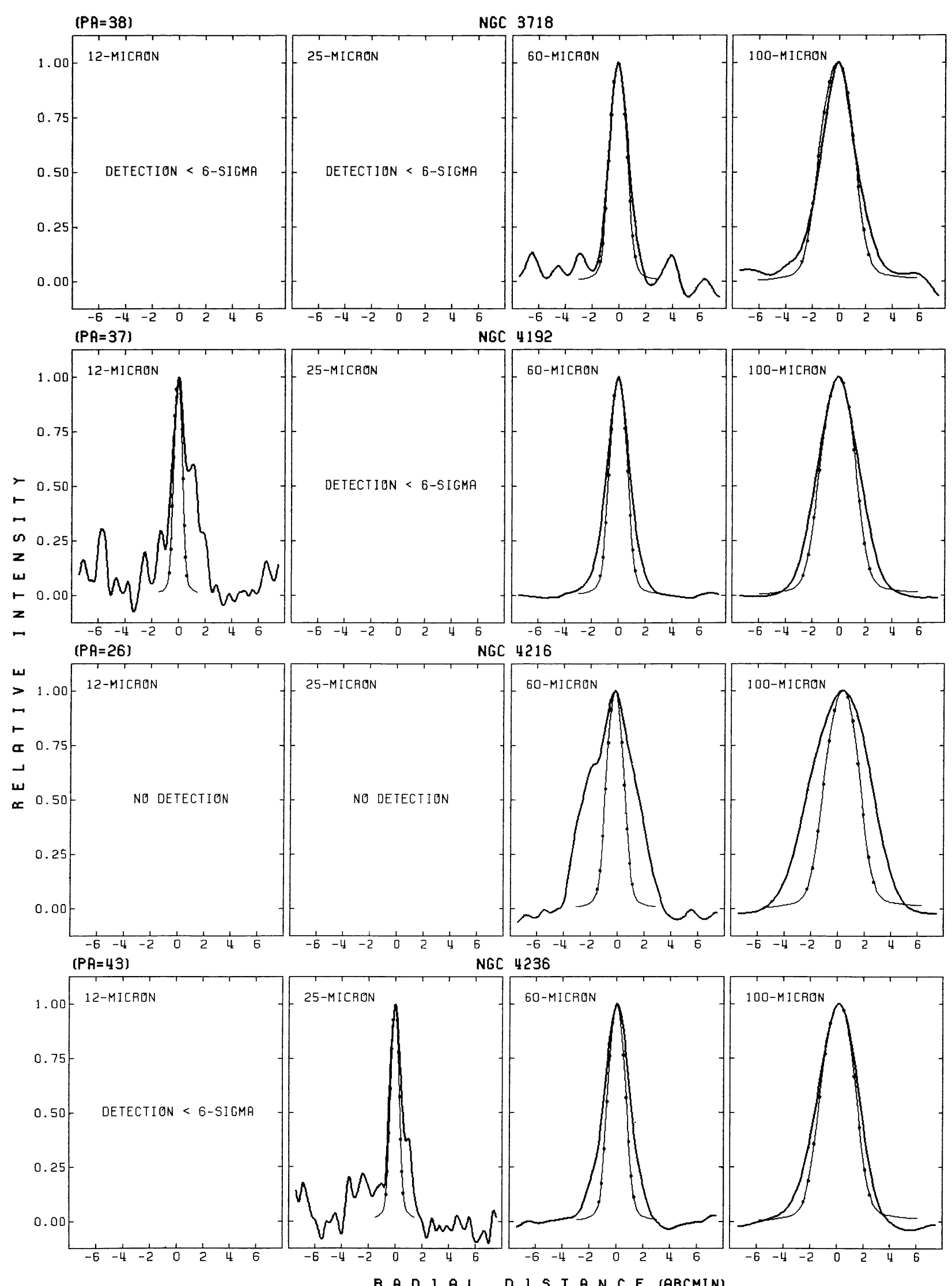

NGC 4236

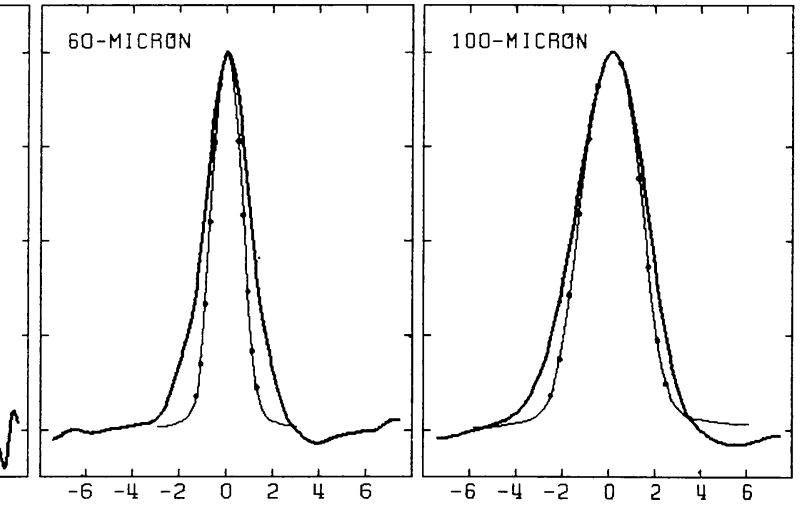

Fig. 1-Continued 
0
$\cdots$
$\vdots$
$\vdots$
0
0
$\vdots$
0
1
0
0
0
$\infty$
$\infty$
0
7
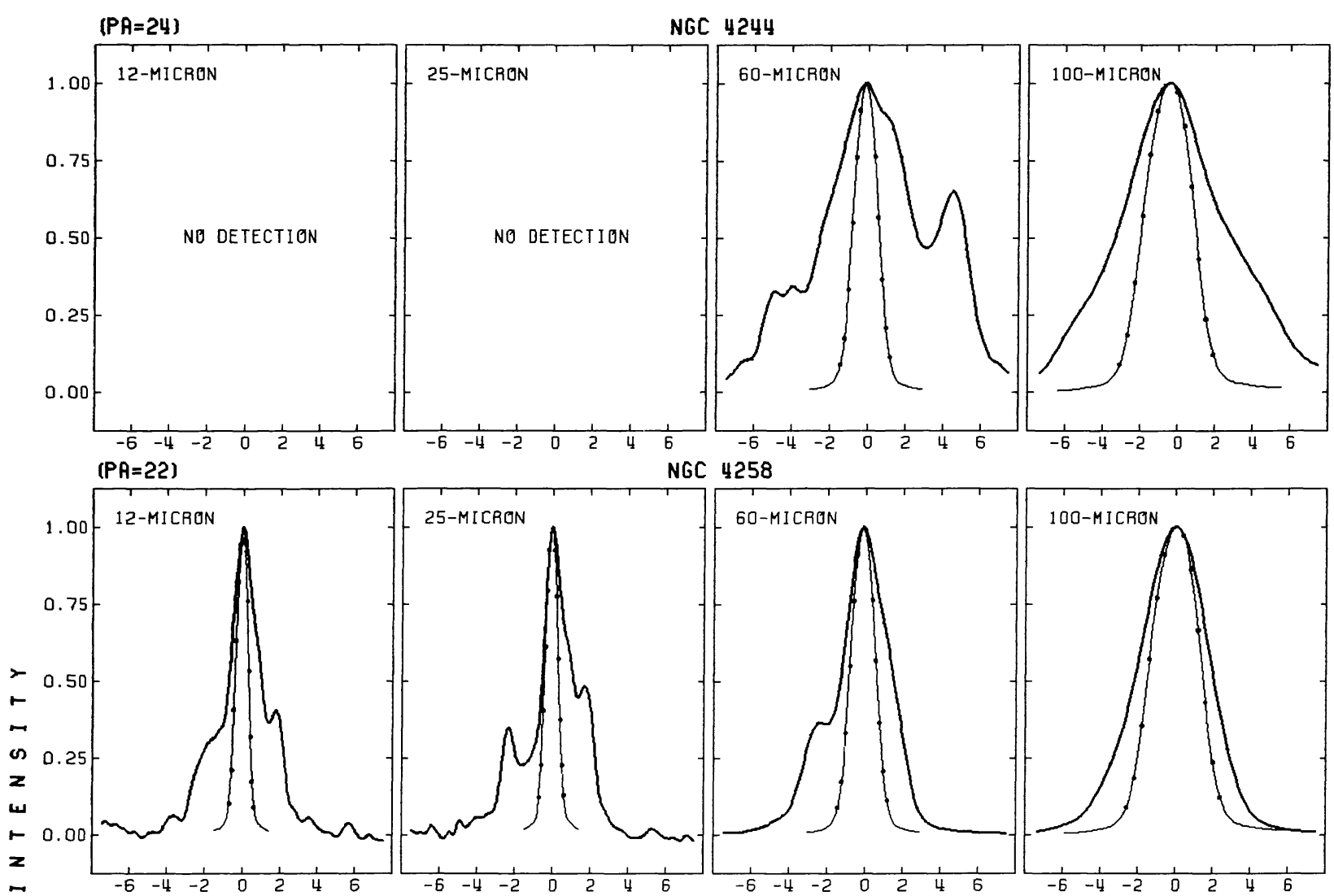

NGC 4258
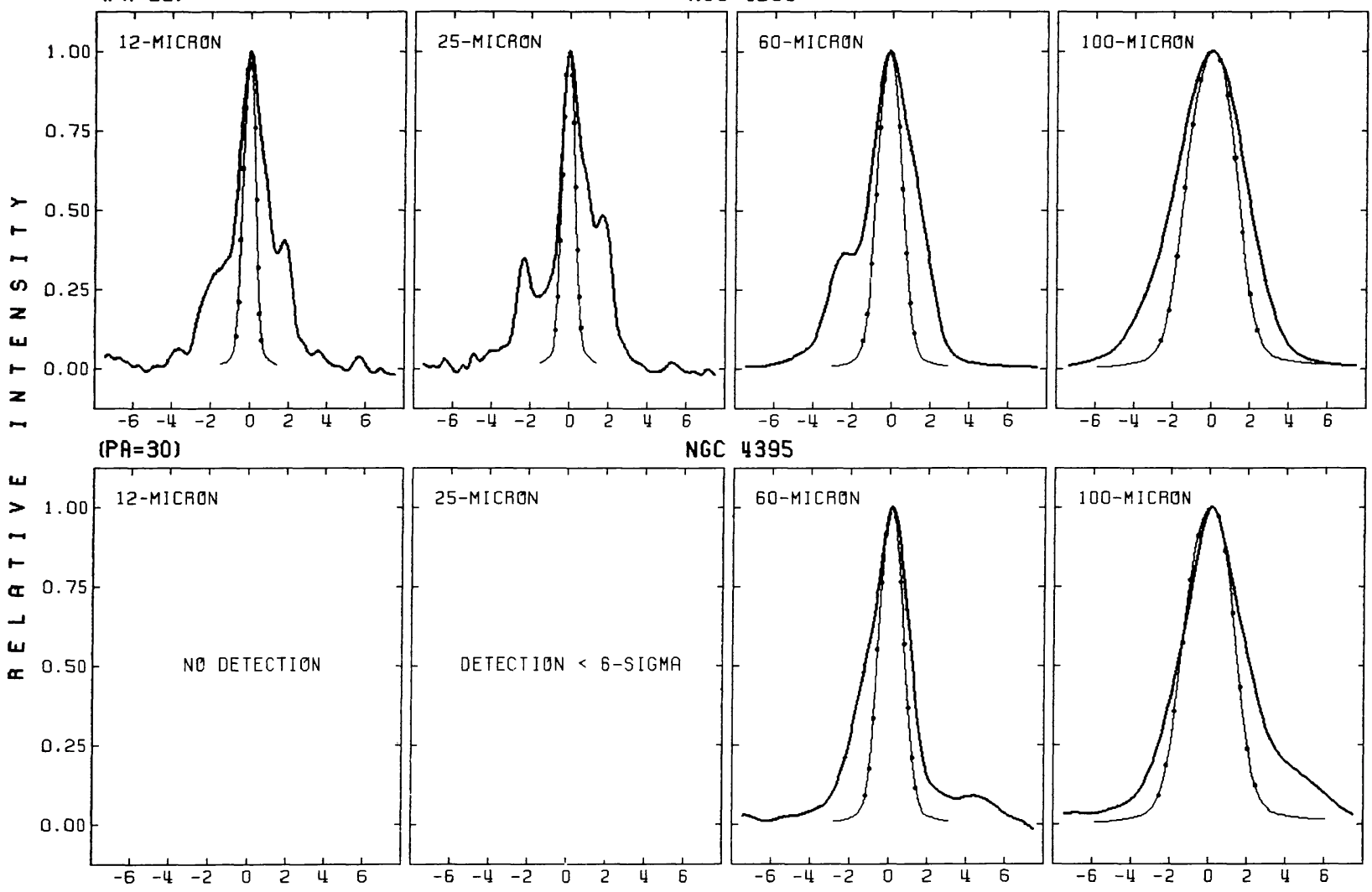

NGC 4395
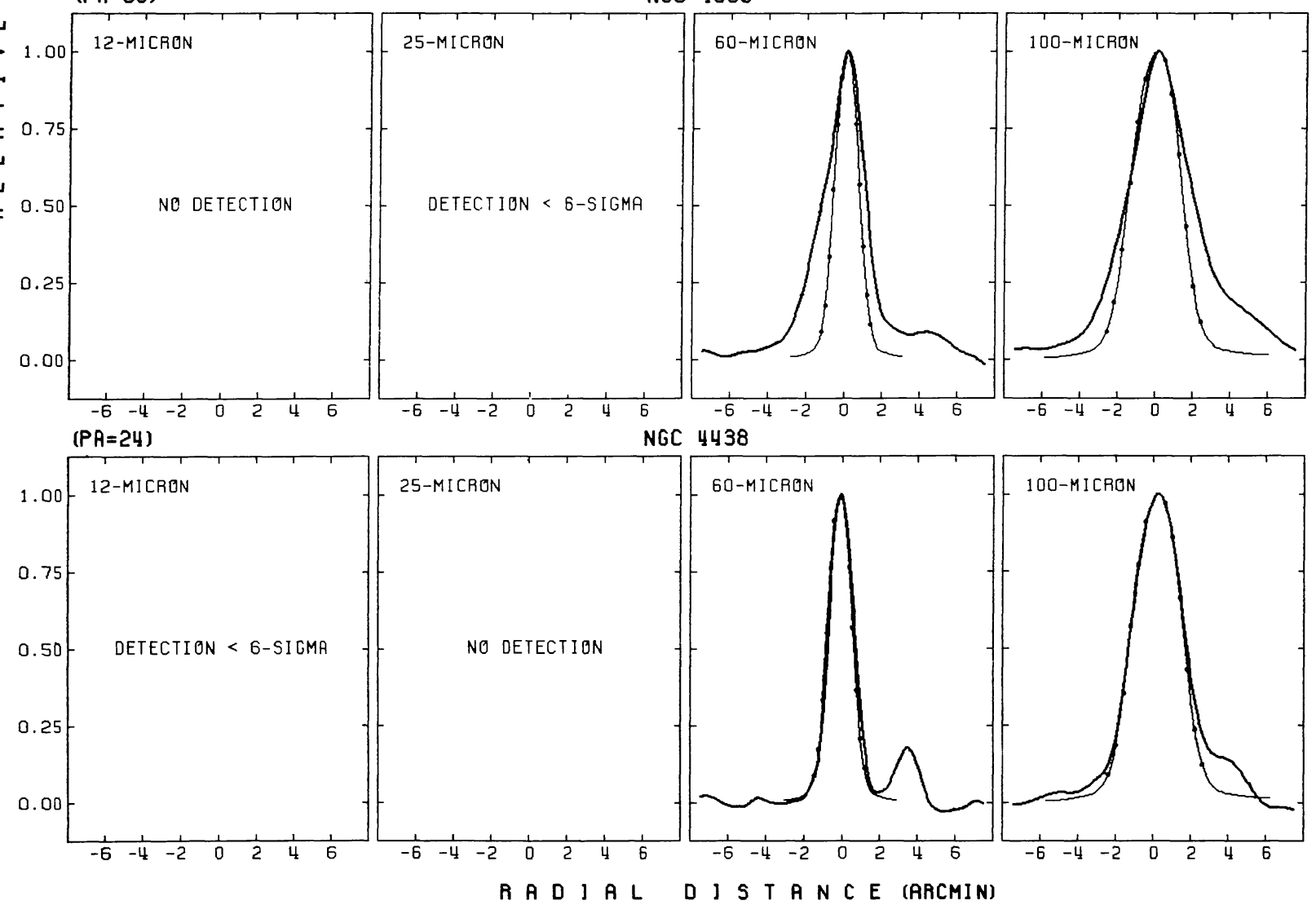

FIg. 1-Continued 
0
$\cdots$
$\vdots$
$\vdots$
0
0
$\vdots$
0
1
0
0
0
$\infty$
$\infty$
0
7
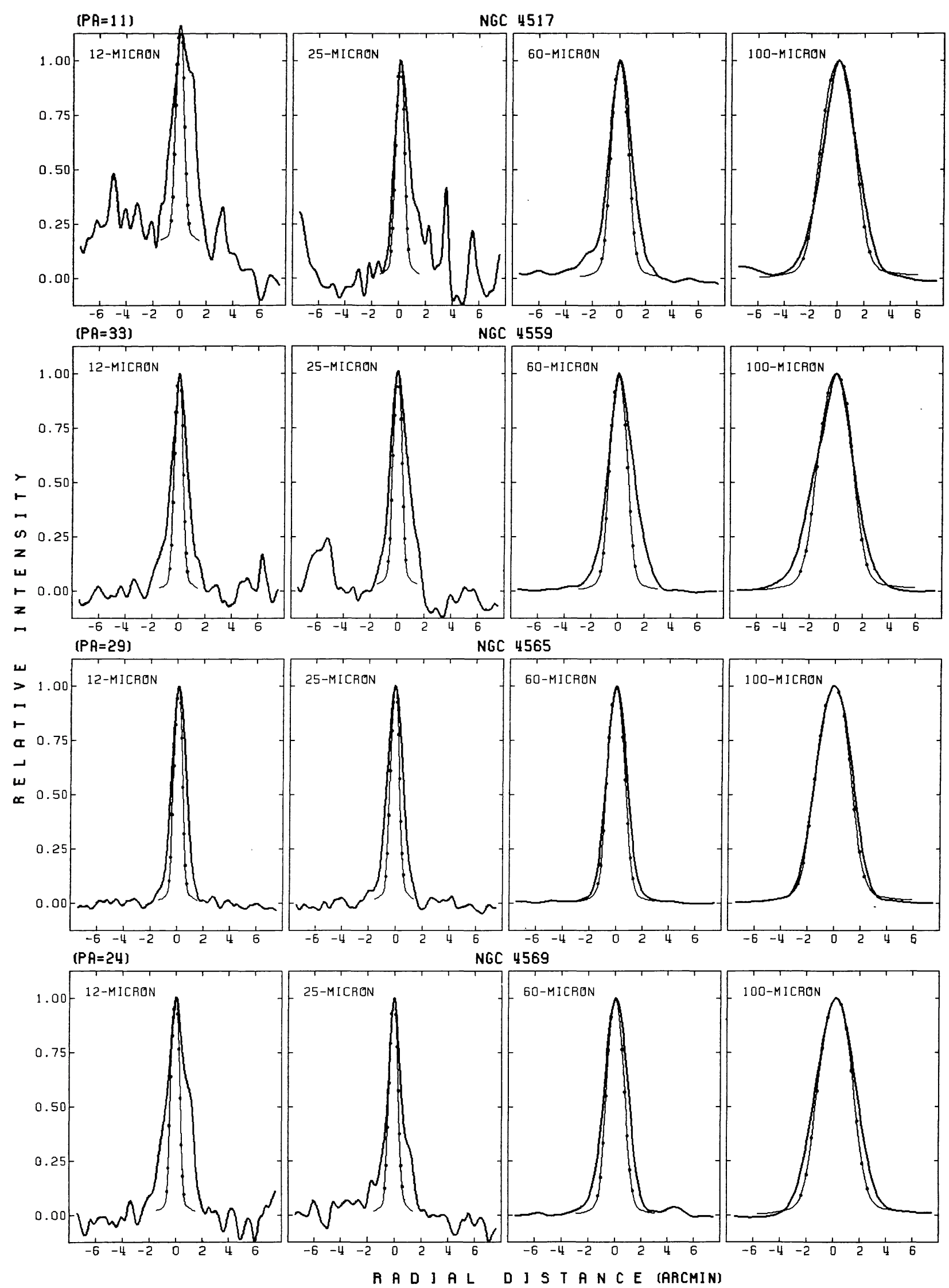

FIG. 1-Continued 


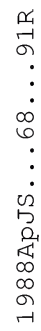

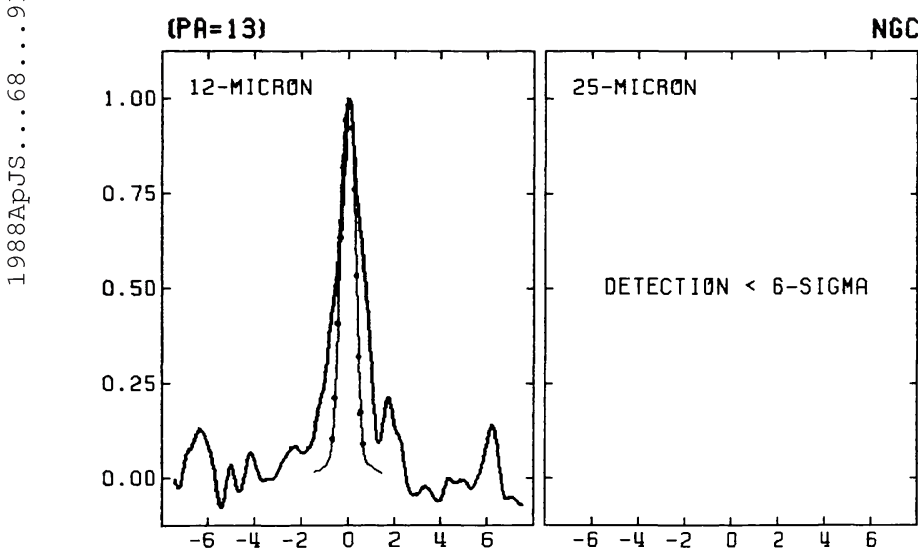

NGC 4594
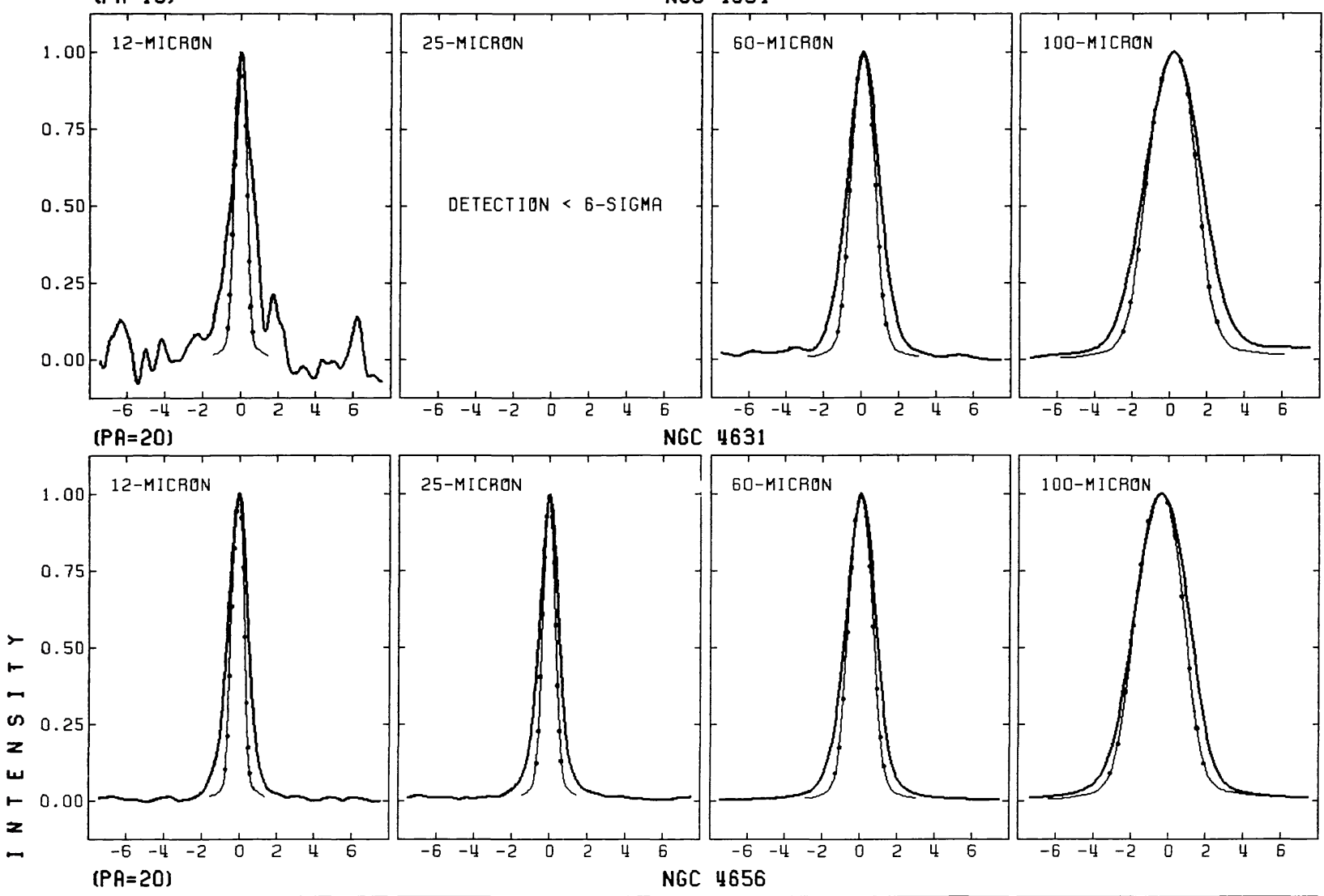

4631
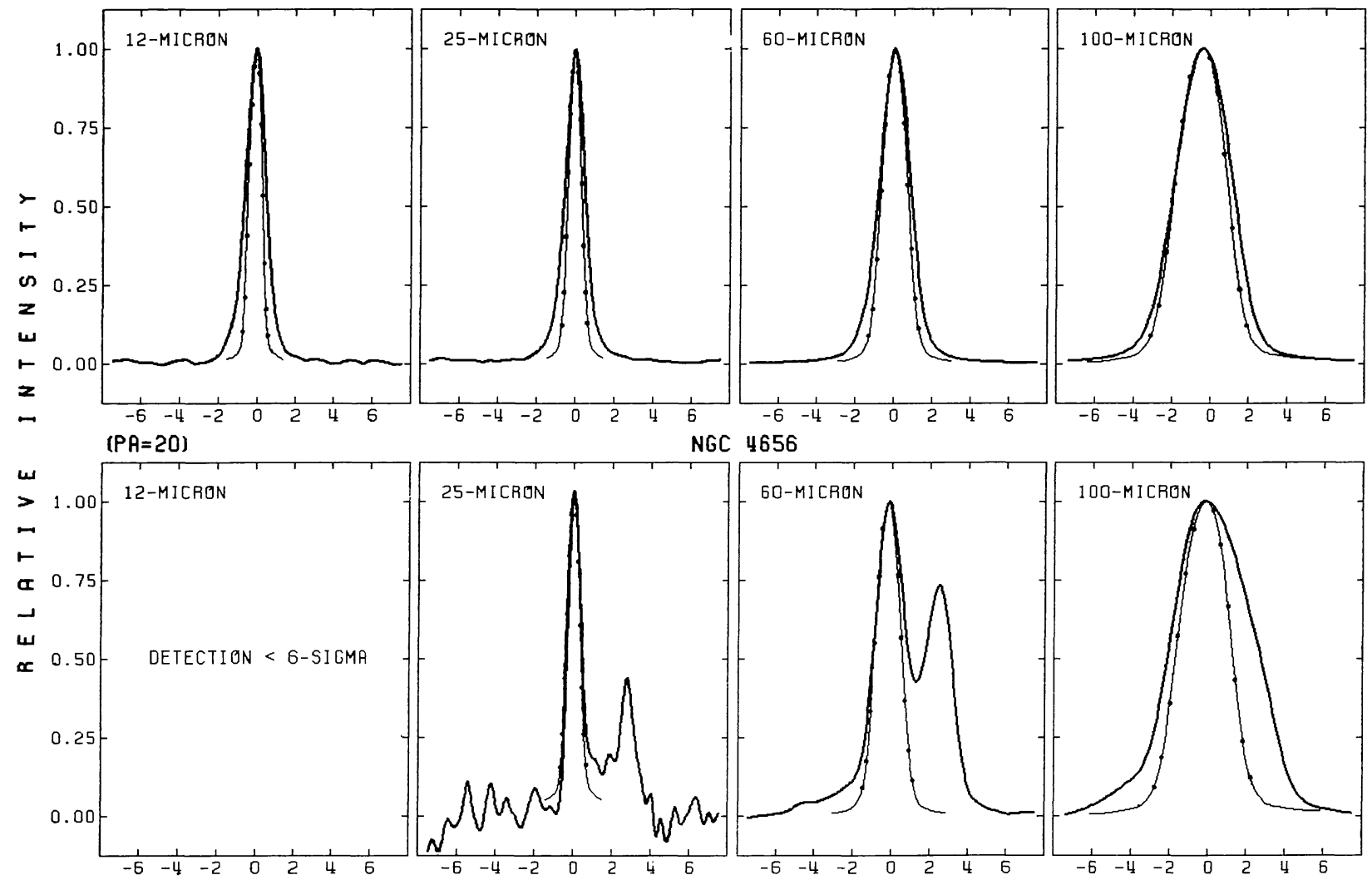

$$
(P A=27)
$$
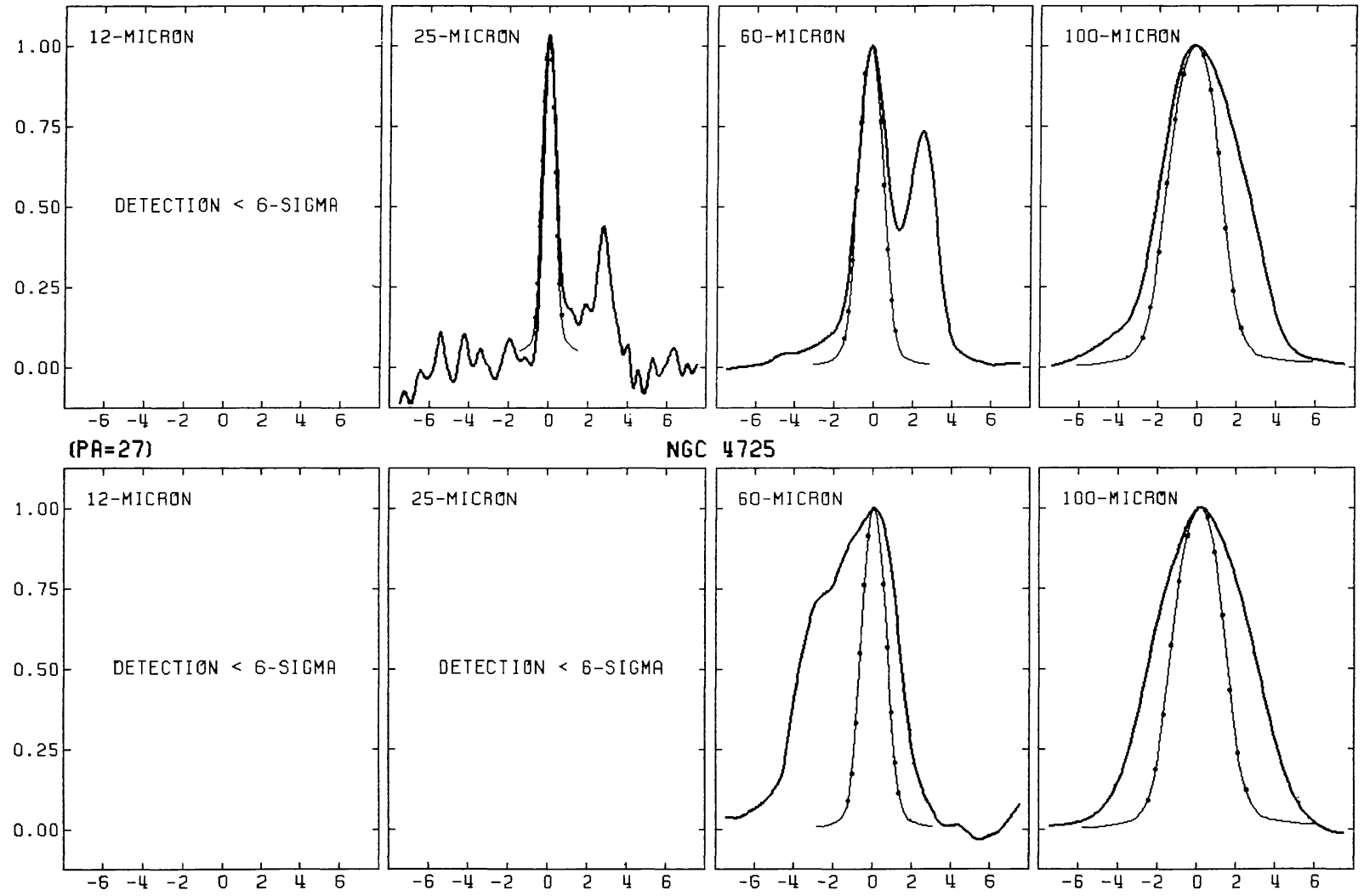

NGC 4725

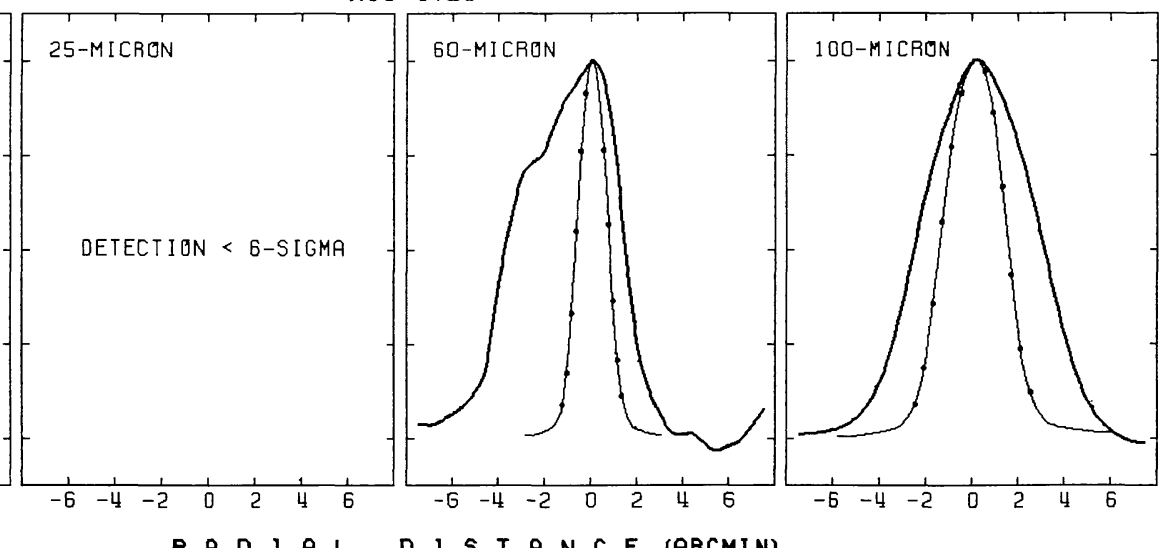

FIG. 1-Continued 
0
$\cdots$
$\vdots$
$\vdots$
0
0
$\vdots$
0
1
0
0
0
$\infty$
$\infty$
0
7
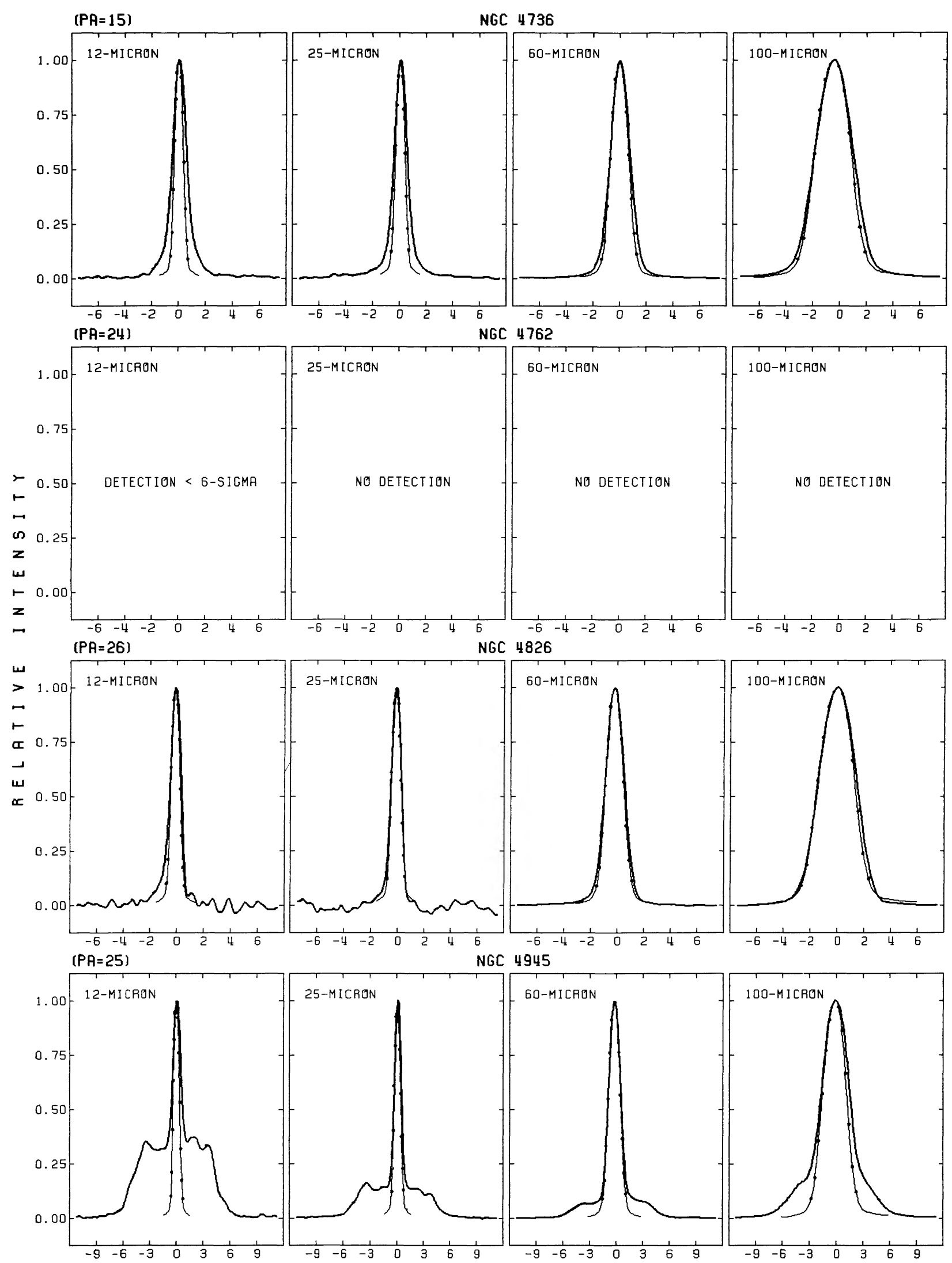

4945

R A D I A L D J T T N C E (ARCMIN)

FIG. 1-Continued 


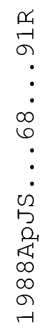
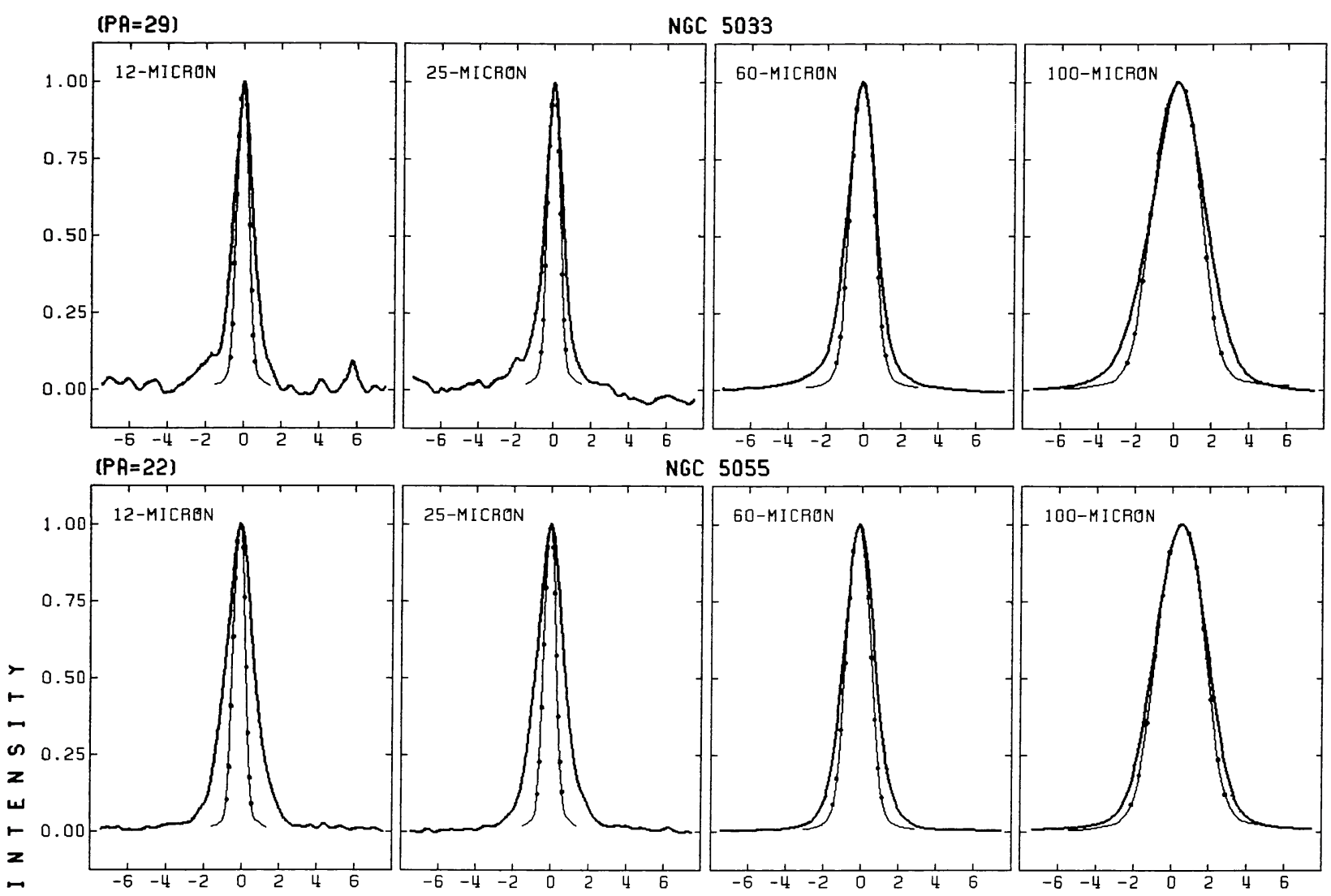

GC 5055
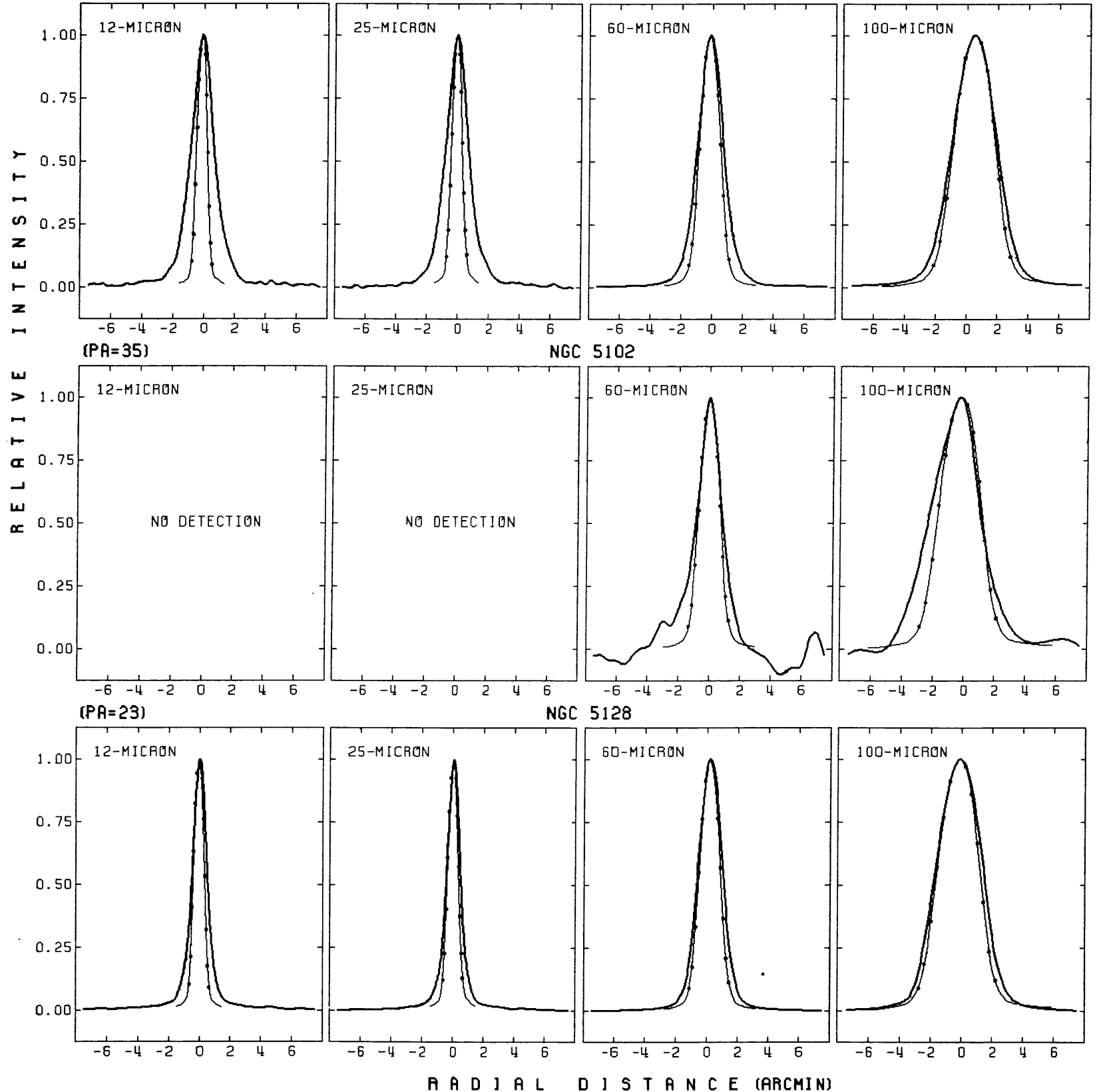

FIG. 1-Continued 


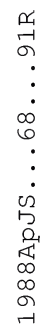
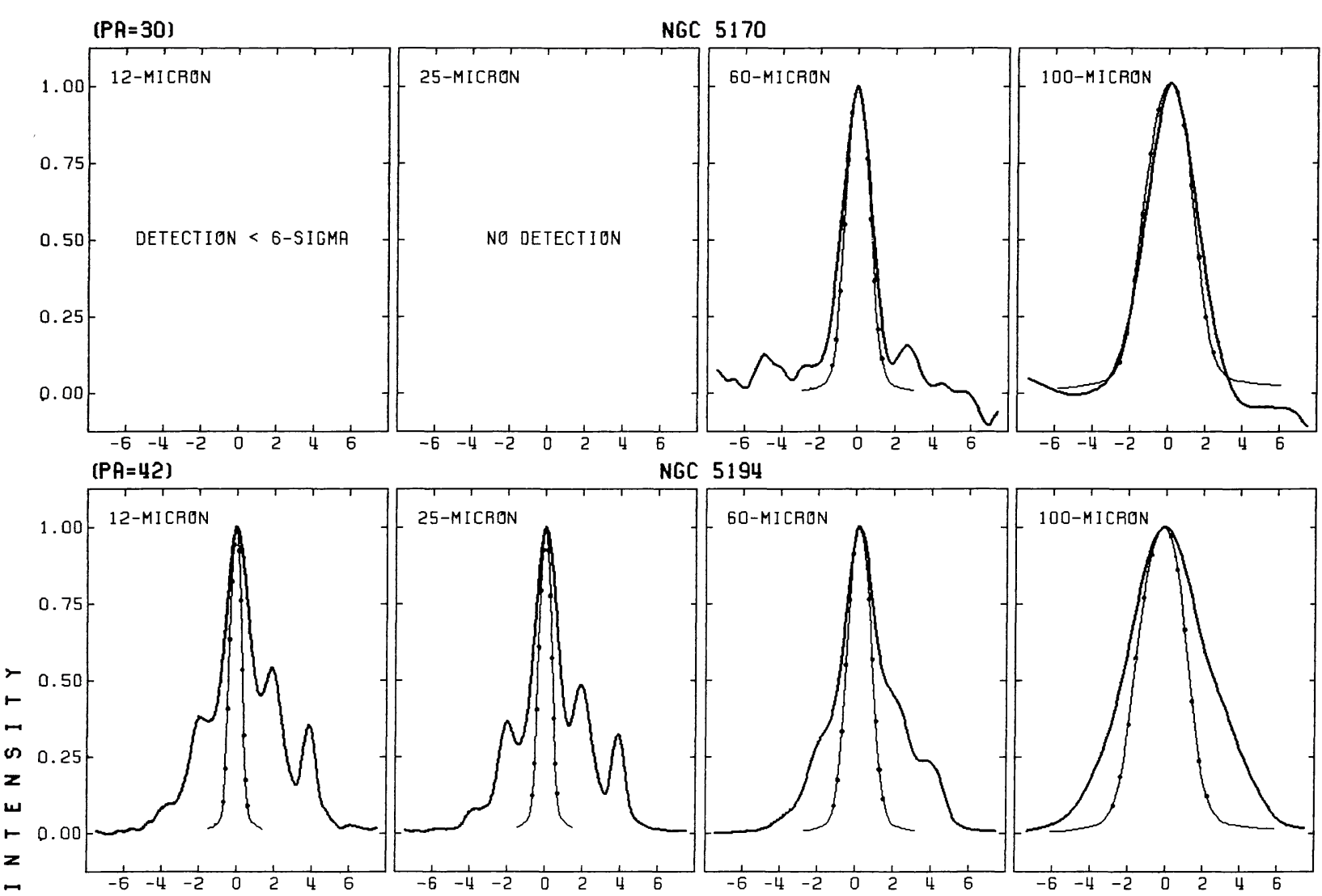

5194
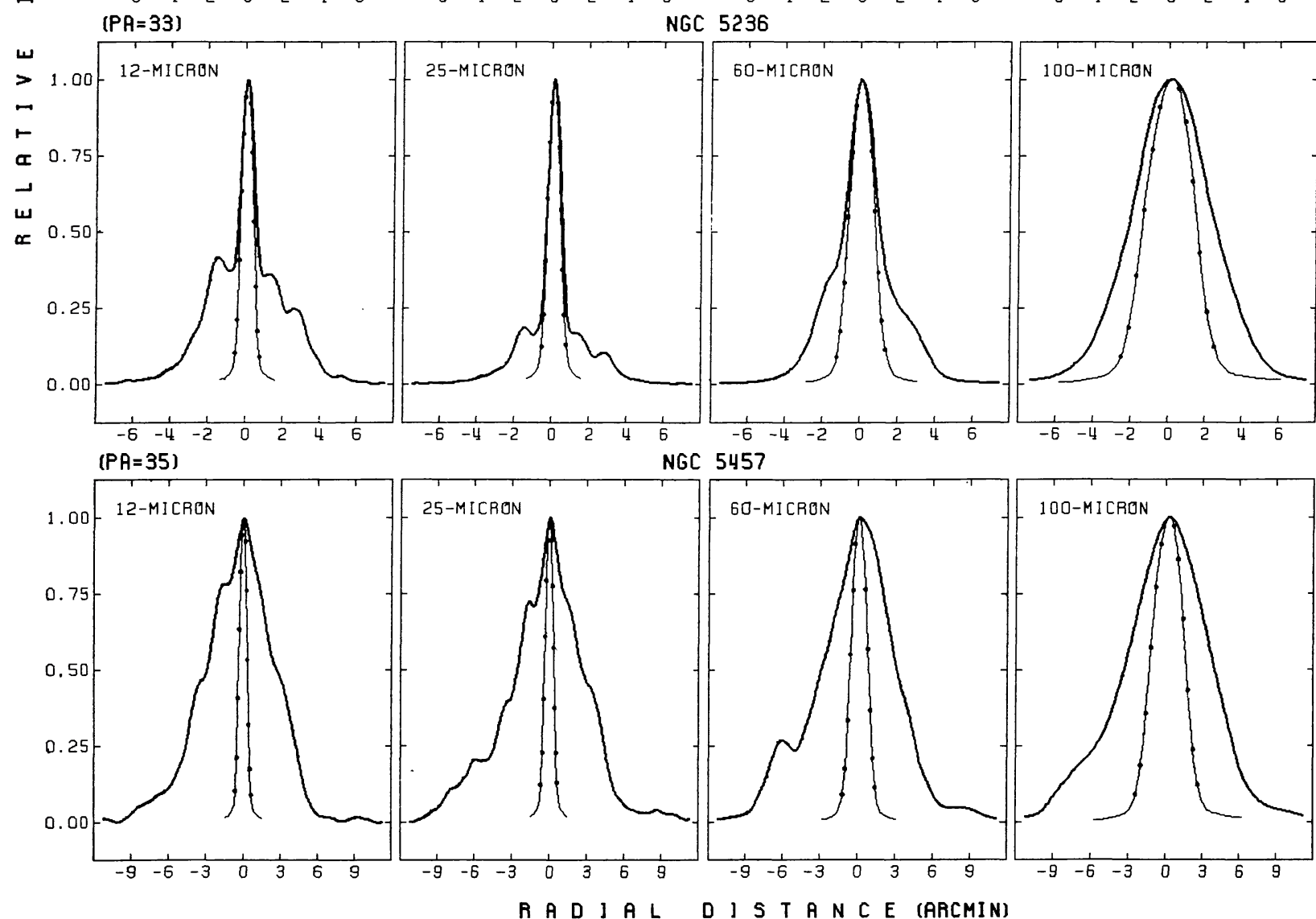

FIG. 1-Continued 


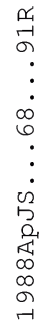
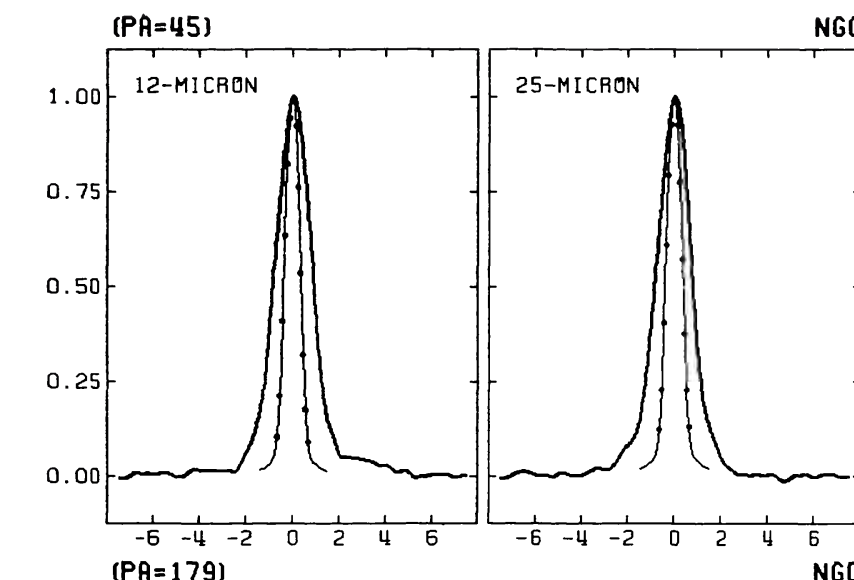

NGC 5907
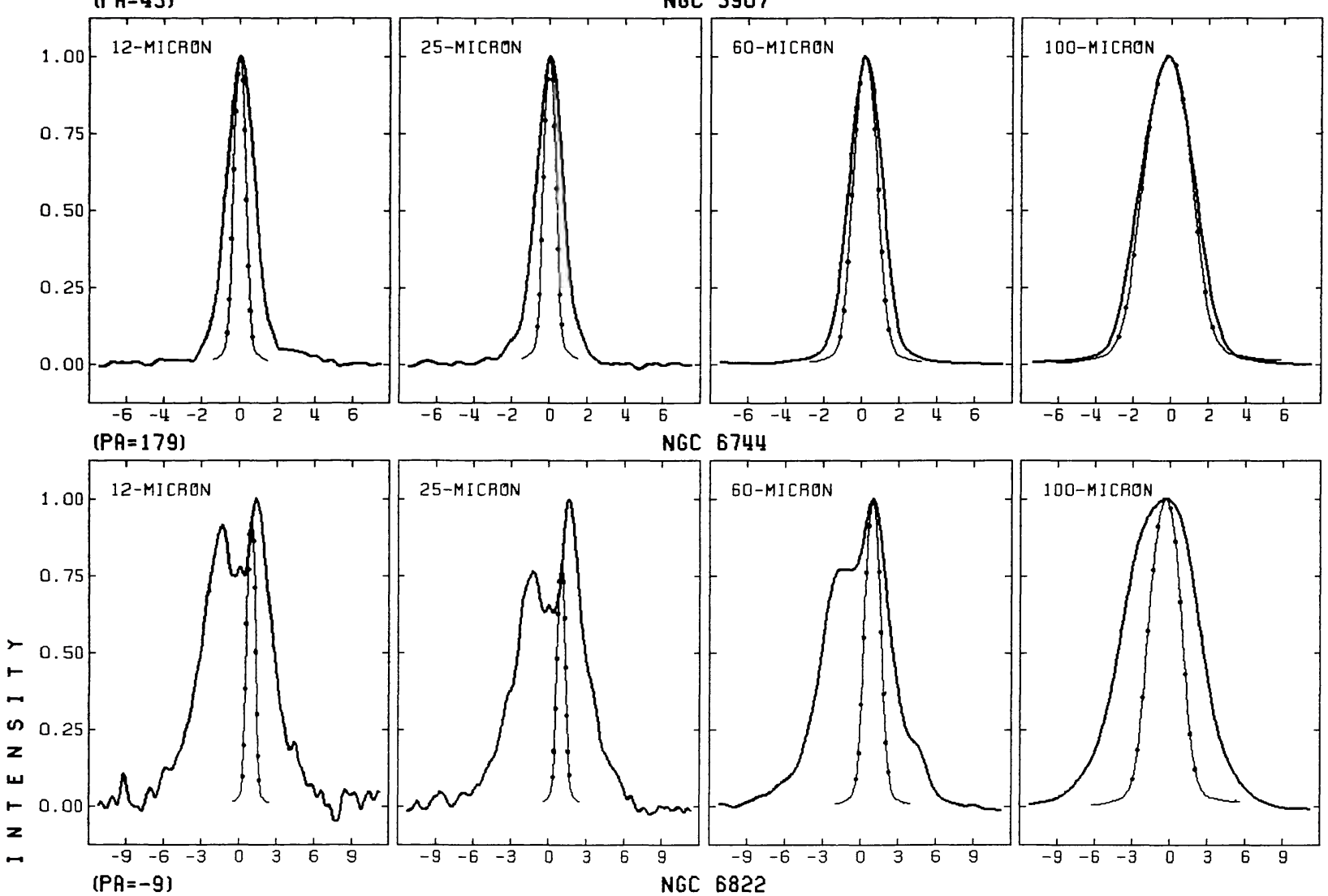

6744
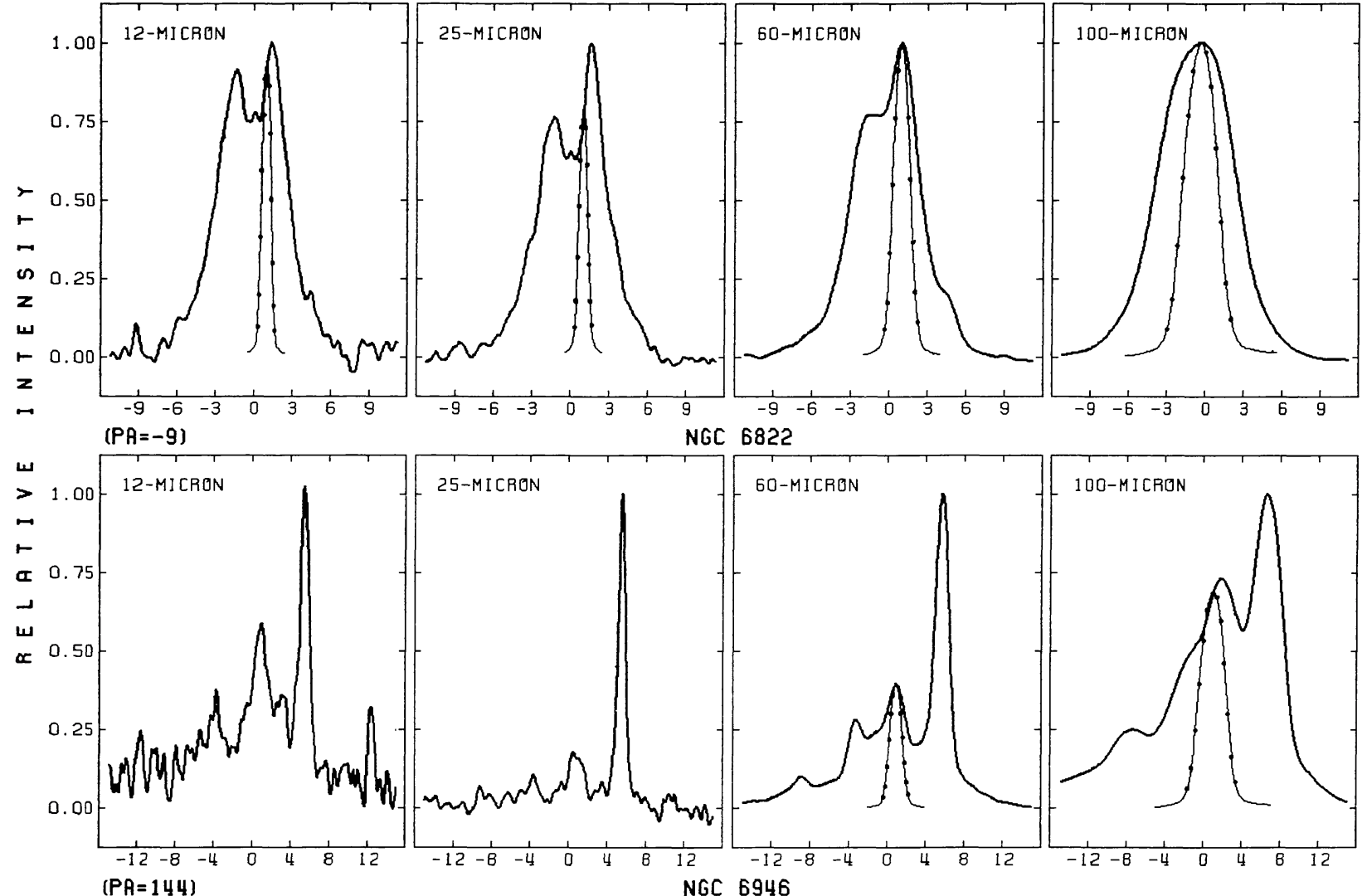

6822
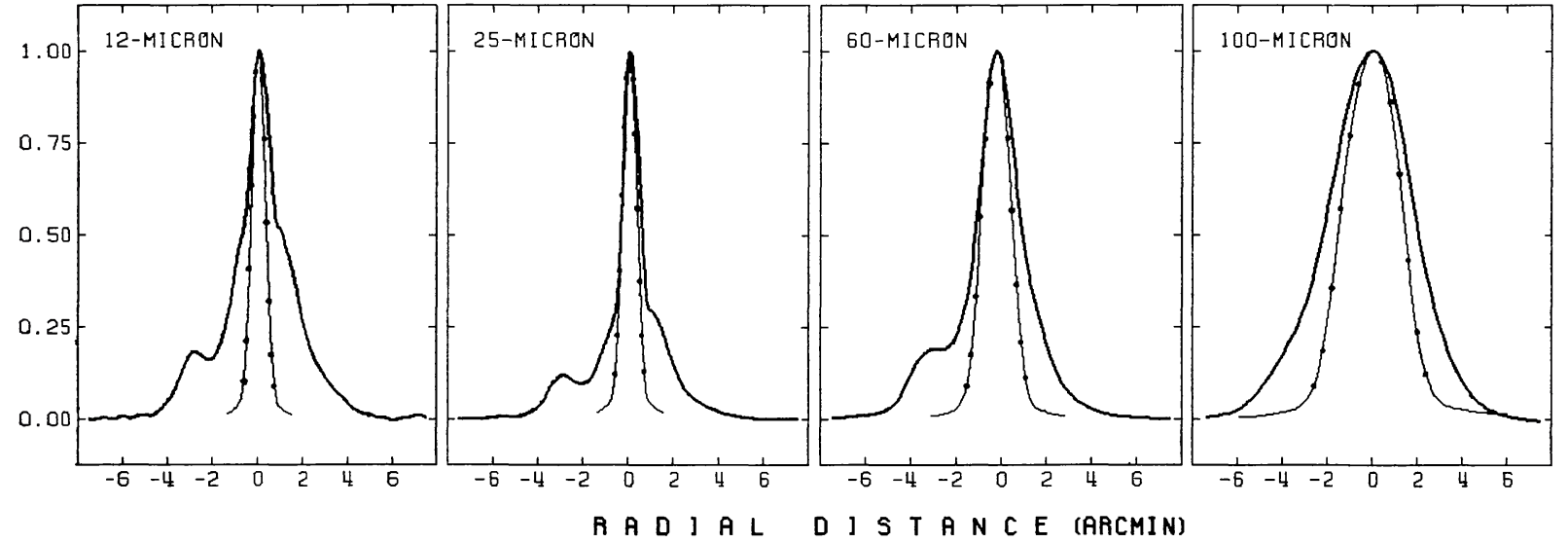

FIG. 1-Continued 
0
$\cdots$
$\vdots$
$\vdots$
0
0
$\vdots$
0
1
0
0
0
$\infty$
$\infty$
0
7

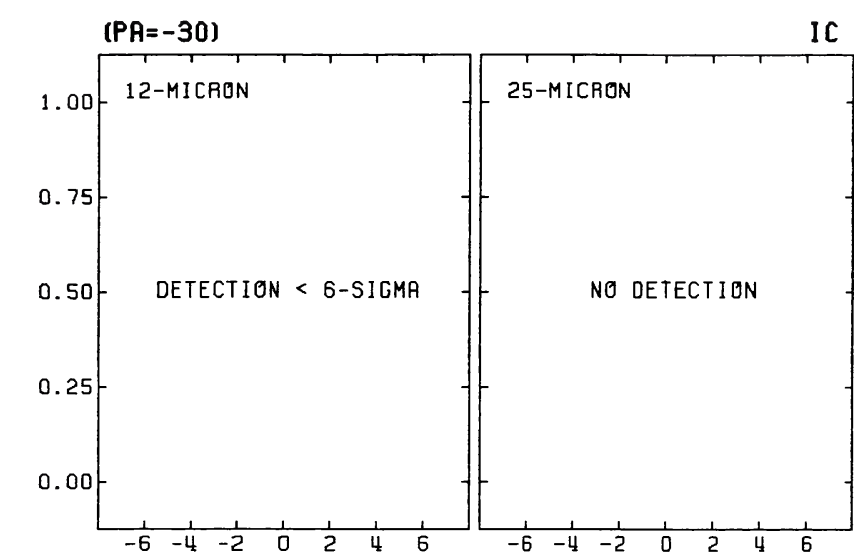

5201

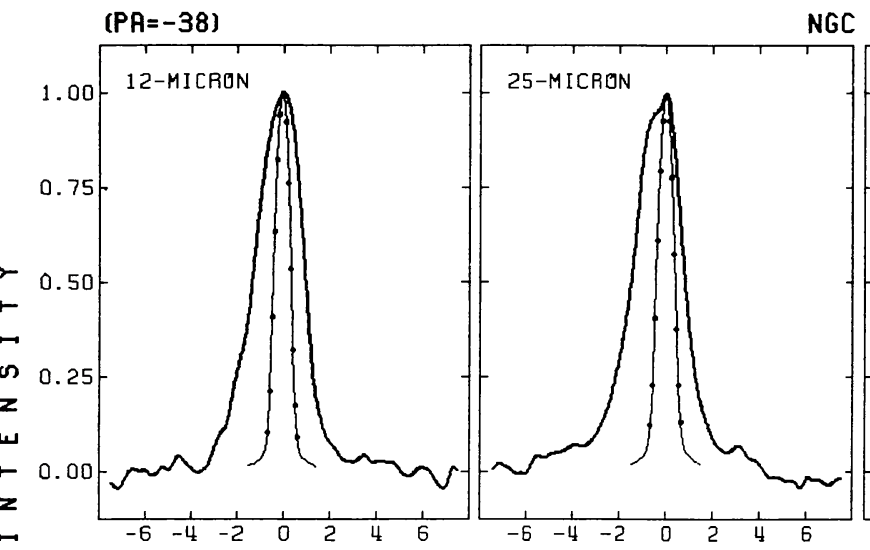

NGC 7331
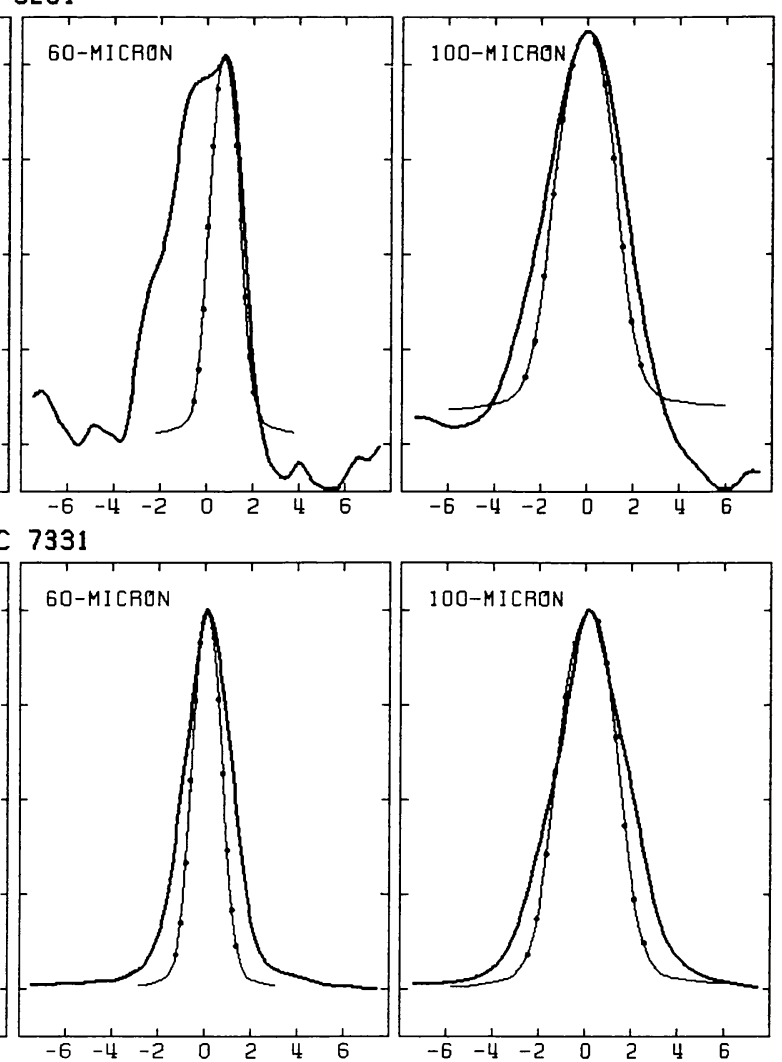

$(P A=-30) \quad$ NGC 7640
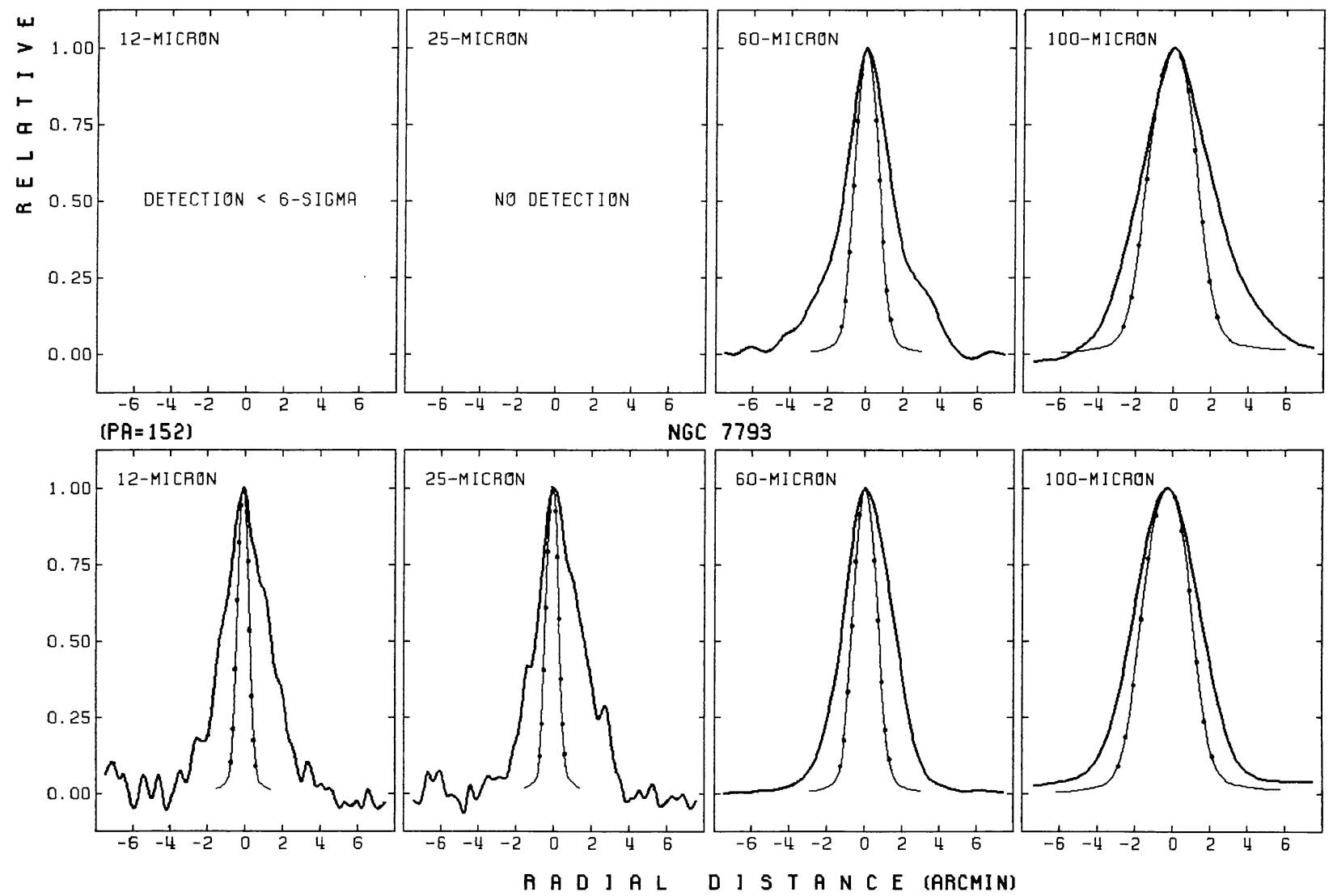

FIG. 1-Continued 


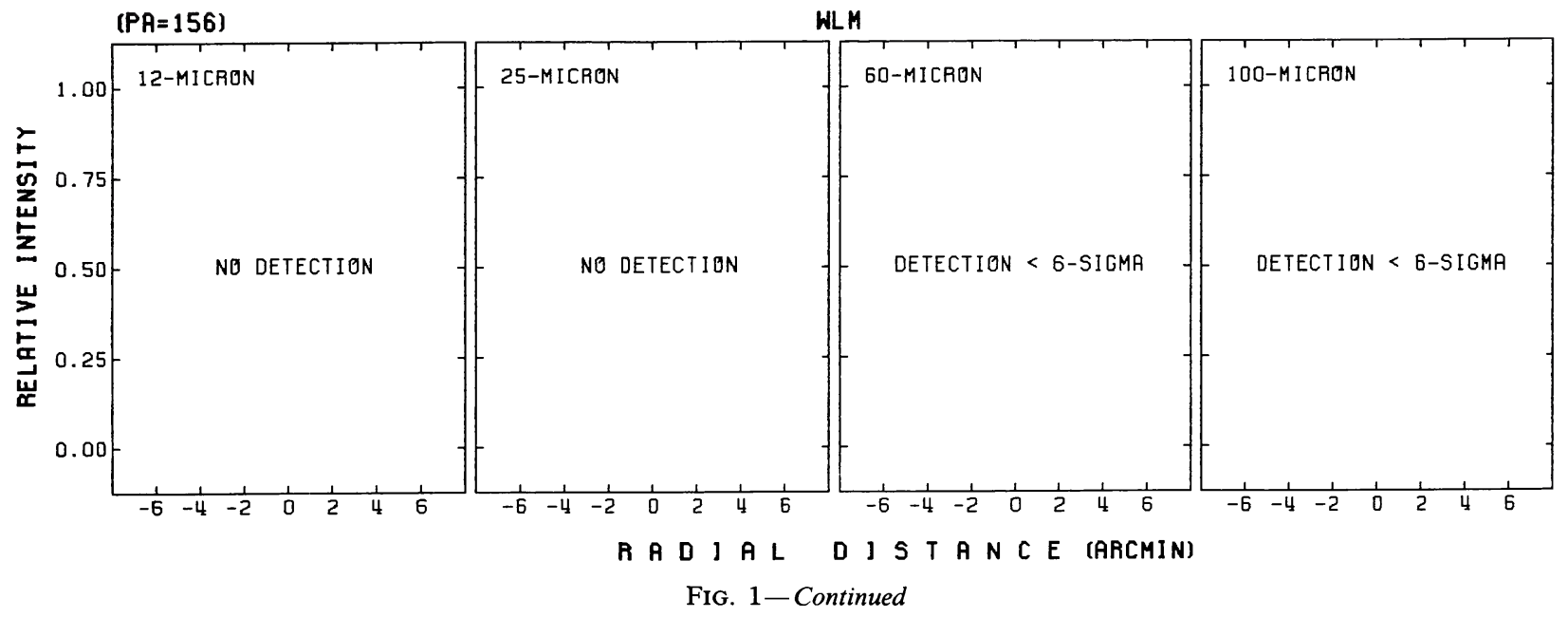

(i.e., they are the "earliest" type galaxies at $60 \mu \mathrm{m}$ ). Disk emission is seen in each case, but the pointlike nuclear source is very dominant, while the faint "disk" resembles a plateau.

Group 2: NGC 55, NGC 2403, NGC 3031 (M81), NGC 4258, $N G C 5194$ (M51). - These galaxies are of "later" $60 \mu \mathrm{m}$ type than group 1 galaxies, exhibiting a less strongly enhanced nuclear source relative to the disk emission. The nuclear source is typically not pointlike, and blends more smoothly into the disk emission. In the larger galaxies spiral arm structure has been resolved.

Group 3: NGC 224 (M31), NGC 247, NGC 300, NGC. 598 (M33), NGC 1313, NGC 4244, NGC 4725, NGC 5457 (M101), $N G C 6744$. - The $60 \mu \mathrm{m}$ profiles of these nine galaxies are still nucleus-dominated but show less concentration toward the nuclear regions than group 2 galaxies, often having an almost triangular shape. Structure in the outer regions of the galaxies is frequently apparent. The peculiar galaxy NGC 1313 is double-peaked at 12,25 , and $60 \mu \mathrm{m}$.

Group 4: $S M C, L M C, I C$ 2574, NGC 4236, NGC 4395, $N G C$ 6822. - These are "late-type" $60 \mu \mathrm{m}$ galaxies in which the IRAS images are dominated by emission from prominent $\mathrm{H}$ II region complexes. There is little or no enhanced IRAS emission from the nuclear region.

Group 5: NGC 253, NGC 1448, NGC 3109, NGC 3628, $N G C$ 4565, NGC 5055.-For these highly inclined galaxies the IRAS scanning direction was very close to the galaxy minor axis. Little information is available on their far-infrared structure.

\section{d) Comments on the Atlas Maps}

In the following, brief descriptions of the IRAS appearance of the atlas galaxies that exhibit particularly interesting infrared structure are given. Caveats in the interpretation of the data are also noted.

$N G C 55$.- The IRAS images of this edge-on galaxy are dominated by three pointlike sources including the nucleus, all of which are seen at both 25 and $60 \mu \mathrm{m}$ and two of which are seen at $12 \mu \mathrm{m}$. The $100 \mu \mathrm{m}$ beam is too large to resolve either of the off-nuclear point sources. The off-nuclear sources appear to coincide with dust lanes or spiral arms (viewed edge-on) in the optical image.
The orientation of the nuclear point source is affected by the beam scanning direction, and the outer contours of the 60 $\mu \mathrm{m}$ map to the northeast are probably distorted by emission from a small group of background galaxies.

NGC 224 (M31). - The IRAS images of M31 have been described previously by Habing et al. (1984), Soifer et al. (1986), and Walterbos and Schwering (1987). The newly processed data presented here show the same features: the galaxy is well resolved, with a nuclear source and a bright infrared ring that corresponds to prominent dust lanes and the galaxy $\mathrm{H} \mathrm{I}$ and $\mathrm{CO}$ rings.

NGC 205 is seen in the 12, 60, and $100 \mu \mathrm{m}$ maps, and M32 is seen in the 12 and $25 \mu \mathrm{m}$ maps.

$N G C 253$. - The images of this very bright galaxy are very much dominated by reflected emission from the IRAS telescope spider. A "hysteresis tail," caused by electronic relaxation effects of the IRAS detectors after crossing a bright source (see the IRAS Supplement, § IV.A.7), is seen in the forward scanning direction. Because of these effects, useful information is available only along the major axis.

$S M C$ and $L M C$. - The maps of the SMC and LMC displayed in the atlas were constructed from all-sky survey scans. The 12, 25, and $60 \mu \mathrm{m}$ maps have been smoothed to the resolution of the $100 \mu \mathrm{m}$ map $\left(\sim 3^{\prime} \times 5^{\prime}\right)$.

The infrared emission structure of the Magellanic Clouds is typical of group 4 galaxies. Diffuse emission from the galaxy disk is seen, but the infrared images are dominated by localized emission sources coincident with $\mathrm{H}$ II regions and optical emission knots seen in the red-light photographs. Diffuse emission at 12 and $25 \mu \mathrm{m}$ is seen along the optical axial bar of both galaxies.

NGC 598 (M33).- The IRAS maps of M33, along with those of the other Local Group members-the Magellanic Clouds and M31 - show the most detailed structure of all the galaxies observed. Diffuse emission from the disk and prominent localized sources coincident with the galaxy's brightest $\mathrm{H}$ II region complexes are seen in the infrared images. The inner pair of spiral arms is clearly visible in the infrared maps.

IC 342 . - IC 342 is one of the most interesting of the galaxies presented here. Although not completely resolved, the main spiral arms can be easily traced in all but the 100 
$\mu \mathrm{m}$ image. The arms are superposed on a smooth disk, and a pointlike nucleus is seen in both the optical and the infrared images. The spurs to the east and to the west may be due to background objects.

NGC 1448. - The galaxy is unresolved along the in-scan direction which is near the galaxy minor axis, but may be slightly extended at 60 and $100 \mu \mathrm{m}$ along the cross-scan (near the galaxy major axis) direction.
NGC 3031 (M81).-Enhanced infrared emission is coincident with the chains of $\mathrm{H}$ II regions in the spiral arms to the north and south of the nucleus. The $100 \mu \mathrm{m}$ image is affected by Galactic cirrus to the north at the lowest contour levels.

IC 2574. - The prominent 60 and $100 \mu \mathrm{m}$ sources are coincident with the cluster of $\mathrm{H}$ II regions to the northeast of the galaxy center. Faint emission from the disk is also seen in these maps. The $100 \mu \mathrm{m}$ image is affected by Galactic cirrus.

TABLE 8

Optically LaRge Galaxies Listed in Other Catalogs

\begin{tabular}{|c|c|c|c|c|c|c|c|c|}
\hline \multirow{2}{*}{$\begin{array}{c}\begin{array}{c}\text { Catalog } \\
\text { Name } \\
(1)\end{array} \\
\text { U00192 }\end{array}$} & \multirow{2}{*}{$\begin{array}{c}\begin{array}{c}\mathrm{RC} 2 \\
\text { Name } \\
(2)\end{array} \\
10\end{array}$} & \multicolumn{3}{|c|}{$\begin{array}{l}\text { R.A. (1950) Dec. } \\
(3)\end{array}$} & \multirow{2}{*}{$\begin{array}{c}\begin{array}{c}\text { Type } \\
(5)\end{array} \\
d I r r\end{array}$} & \multirow{2}{*}{$\begin{array}{c}\begin{array}{c}T \\
(6)\end{array} \\
10 \mathrm{~B}\end{array}$} & \multirow{2}{*}{$\frac{\begin{array}{c}D_{25}^{*} \\
(7)\end{array}}{8^{\prime} .7}$} & \multirow{2}{*}{$\begin{array}{l}\mathrm{RC2} \\
D_{25} \\
(8)\end{array}$} \\
\hline & & $0^{\mathrm{h}} 17^{\mathrm{m}}$ & $36^{8}$ & $59^{\circ} 02^{\prime}$ & & & & \\
\hline U00452 & No221 & 039 & 58.0 & $403533 \mathrm{D}$ & $\mathrm{E}$ & -6 & 13.7 & 7.6 \\
\hline $\mathrm{E} 411-25$ & N0289 & 050 & 17 & -312836 & $\mathrm{Sb}$ & 4B & 8.6 & 3.7 \\
\hline E351-30 & $A 0057-33$ & 057 & 47 & -335841 & $\mathrm{dE}$ & -5 & 65.4 & \\
\hline U00718 & N0404 & 106 & 39.3 & $\begin{array}{llll}35 & 27 & 10 & \mathrm{D}\end{array}$ & $\mathrm{E} / \mathrm{S} 0$ & $-3 \mathbf{A}$ & 8.0 & 4.4 \\
\hline U00864 & N0474 & 117 & 31.7 & $030917 \mathrm{D}$ & So & $-2 \mathrm{~A}$ & 12.6 & 7.9 \\
\hline U01249 & $\mathrm{I} 1727$ & 144 & 41.6 & $270455 \mathrm{D}$ & & $9 B$ & 8.7 & 6.2 \\
\hline U01466 & No772 & 156 & 35.3 & $184550 \mathrm{D}$ & $\mathrm{Sb}$ & $3 \mathbf{A}$ & 8.7 & 7.1 \\
\hline U02173 & N1055 & 239 & 10.7 & $-01345 \mathrm{D}$ & $\mathrm{Sb}$ & $3 \mathrm{~B}$ & 8.7 & 7.6 \\
\hline U02188 & N1068 & 240 & 06.5 & $-01332 \mathrm{D}$ & $\mathrm{Sb}$ & $3 \mathbf{A}$ & 9.7 & 6.9 \\
\hline M-02-09-042 & $\mathrm{N} 1337^{\mathrm{a}}$ & 325 & 36.0 & -93400 & & & 8.9 & \\
\hline $\mathrm{E} 250-03$ & $\mathrm{~N} 1510$ & 401 & 54 & $-43 \quad 3211$ & S... & -6 & 9.3 & 1.0 \\
\hline E250-04 & N1512 & 402 & 16 & -432911 & $\mathrm{SB}(\mathrm{r}:) \mathrm{b}$ & $1 \mathrm{~B}$ & 13.7 & 4.0 \\
\hline E359-27 & N1532 & 410 & 09 & -330000 & $\mathrm{Sc}$ & $2 B$ & 16.6 & 5.6 \\
\hline E157-20 & N1566 & 418 & 53 & -550323 & Sc & $4 X$ & 10.1 & 7.6 \\
\hline E486-05 & N1744 & 457 & 56 & -260600 & SBd & $7 \mathrm{~B}$ & 12.3 & 6.8 \\
\hline E206-20 & & 640 & 24 & $-50 \quad 5500$ & $\mathrm{dE}$ & -5 & 29.4 & \\
\hline U03851 & N2366 & 723 & 34.2 & $691842 \mathrm{D}$ & Irr & $10 \mathrm{~B}$ & 9.7 & 7.6 \\
\hline U04284 & N2541 & 811 & 01.9 & $491253 \mathrm{D}$ & $\mathrm{Sc}$ & $6 \mathrm{~A}$ & 8.8 & 6.6 \\
\hline U04305 & $\mathrm{A} 0813+70$ & 813 & 53.5 & $705213 \mathrm{D}$ & $\mathrm{d} \operatorname{Irr}$ & 10 & 10.0 & 7.6 \\
\hline U04821 & $\mathrm{N} 2768$ & 907 & 45.2 & $601440 \mathrm{D}$ & $\mathrm{E} / \mathrm{S} 0$ & -5 & 8.6 & 6.3 \\
\hline U04936 & $\mathrm{N} 2805$ & 916 & 17.0 & $641855 \mathrm{D}$ & $\mathrm{Sc}$ & $7 X$ & 8.2 & 6.3 \\
\hline U05387 & N3079 & 958 & 35.4 & $\begin{array}{llll}55 & 5511 & \mathrm{D}\end{array}$ & $\ldots$ & $5 \mathrm{~B}$ & 9.4 & 7.6 \\
\hline U05557 & N3184 & 1015 & 17.7 & $414028 \mathrm{D}$ & $\mathrm{Sc}$ & $6 \mathrm{X}$ & 9.2 & 6.9 \\
\hline U05789 & N3319 & 1036 & 15.2 & $415656 \mathrm{D}$ & $\mathrm{SBc}$ & $6 \mathrm{~B}$ & 8.2 & 6.8 \\
\hline U05840 & N3344 & 1040 & 46.6 & $251110 \mathrm{D}$ & Sc & $4 X$ & 8.2 & 6.9 \\
\hline U05850 & N3351 & 1041 & 19.6 & $115800 \mathrm{D}$ & $\mathrm{SBb}$ & $3 \mathrm{~B}$ & 9.2 & 7.4 \\
\hline U05873 & N3359 & 1043 & 21.1 & $632911 \mathrm{D}$ & $\mathrm{SBc}$ & $5 \mathrm{~B}$ & 8.7 & 6.8 \\
\hline U05882 & N3368 & 1044 & 06.9 & $120505 \mathrm{D}$ & $\mathrm{Sa} / \mathrm{Sb}$ & $2 X$ & 8.2 & 7.1 \\
\hline U05986 & N3432 & 1049 & 42.7 & $365305 \mathrm{D}$ & $\cdots$ & $9 \mathrm{~B}$ & 8.2 & 6.2 \\
\hline E572-20 & N3981 & 1153 & 34 & -193700 & $\mathrm{Sc}$ & $4 \mathrm{~A}$ & 9.3 & 3.9 \\
\hline U06937 & N3992 & 1155 & 01.0 & $53 \quad 3913 \quad \mathrm{D}$ & $\mathrm{SBb}$ & $4 B$ & 9.0 & 7.6 \\
\hline E572-47 & N4038 & 1159 & 19 & -183511 & S... & $9 \mathrm{~B}$ & 11.5 & 2.6 \\
\hline E572-48 & N4039 & 1159 & 20 & -183623 & S... & $9 \mathrm{~A}$ & 10.1 & 3.2 \\
\hline U07118 & $\mathrm{N} 4125$ & 1205 & 37.7 & $652703 \mathrm{D}$ & $\mathrm{E}$ & -5 & 8.0 & 5.1 \\
\hline U07183 & N4157 & 1208 & 34.6 & $504551 \mathrm{D}$ & $\mathrm{Sb}$ & $3 \mathrm{X}$ & 8.4 & 6.9 \\
\hline U07278 & N4214 & 1213 & 08.8 & $363619 \mathrm{D}$ & Irr & $10 X$ & 11.7 & 7.9 \\
\hline U07377 & $\mathrm{N} 4274$ & 1217 & 20.2 & $295333 \mathrm{D}$ & $\mathrm{SBa}$ & $2 \mathrm{~B}$ & 8.0 & 6.9 \\
\hline U07508 & $\mathrm{N} 4382$ & 1222 & 53.2 & $182803 \mathrm{D}$ & So & $-1 \mathrm{~A}$ & 9.7 & 7.1 \\
\hline U07532 & $\mathrm{N} 4406$ & 1223 & 39.7 & $131325 \mathrm{D}$ & $\mathrm{E}$ & -5 & 14.8 & 7.4 \\
\hline U07654 & N4486 & 1228 & 17.8 & $123958 \mathrm{D}$ & $\mathrm{E}$ & -4 & 9.2 & 7.2 \\
\hline U07718 & N4526 & 1231 & 30.4 & $075833 \mathrm{D}$ & So & $-2 \mathrm{X}$ & 9.2 & 7.2 \\
\hline U07727 & N4535 & 1231 & 47.9 & $082825 \mathrm{D}$ & $\mathrm{Sc} / \mathrm{SBc}$ & $5 X$ & 8.5 & 6.8 \\
\hline U07878 & N4636 & 1240 & 16.6 & $025743 \mathrm{D}$ & $\mathrm{E}$ & -5 & 9.2 & 6.2 \\
\hline U07898 & N4649 & 1241 & 09.0 & $114923 \mathrm{D}$ & $\mathrm{E}$ & -5 & 9.2 & 7.2 \\
\hline
\end{tabular}


TABLE 8-Continued

\begin{tabular}{|c|c|c|c|c|c|c|c|c|c|}
\hline $\begin{array}{c}\text { Catalog } \\
\text { Name } \\
(1)\end{array}$ & $\begin{array}{c}\mathrm{RC2} \\
\text { Name } \\
(2)\end{array}$ & & & )$^{A .(15}$ & $\begin{array}{r}\text { 950) Dec. } \\
\text { (4) }\end{array}$ & $\begin{array}{c}\text { Type } \\
(5)\end{array}$ & $\begin{array}{c}T \\
(6)\end{array}$ & $\begin{array}{l}D_{25}^{*} \\
(7)\end{array}$ & $\begin{array}{l}\mathrm{RC} 2 \\
D_{25} \\
(8)\end{array}$ \\
\hline U08286 & N5023 & $13^{h}$ & $09^{m}$ & ${ }^{n} 58^{8} .0$ & $44^{\circ} 18^{\prime} 13^{\prime \prime} \mathrm{D}$ & $\mathrm{Sc}$ & 5 & $8 ! 2$ & 6.5 \\
\hline E508-48 & N5078 & 13 & 17 & 05 & -270848 & So & $1 \mathrm{~A}$ & 43.4 & 3.2 \\
\hline E576-33 & N5084 & 13 & 17 & 34 & -213353 & SO? & -2 & 14.5 & 4.8 \\
\hline E270-17 & & 13 & 31 & 39 & -451706 & S.../I & 7 & 17.5 & \\
\hline U08745 & N5322 & 13 & 47 & 35.1 & $602621 \mathrm{D}$ & $\mathrm{E}$ & -5 & 8.0 & 5.5 \\
\hline U09499 & N5746 & 14 & 42 & 24.2 & $20953 \mathrm{D}$ & $\mathrm{Sb}$ & $3 X$ & 8.1 & 7.9 \\
\hline U09631 & N5792 & 14 & 55 & 48.2 & $-05326 \mathrm{D}$ & $\mathrm{SBb}$ & $3 \mathrm{~B}$ & 8.7 & 7.2 \\
\hline U09723 & N5866 & 15 & 05 & 07.8 & $\begin{array}{llll}55 & 57 & 16 & \mathrm{D}\end{array}$ & So & $-1 \mathrm{~A}$ & 8.6 & 5.2 \\
\hline E274-01 & & 15 & 10 & 47 & -463736 & Sc: & 6 & 15.6 & \\
\hline U09792 & & 15 & 13 & 30 & 003 & $\mathrm{dE}$ & -5 & 15.6 & \\
\hline U10359 & N6140 & 16 & 20 & 36.0 & $653030 \mathrm{D}$ & $\mathrm{SBc}$ & $5 \mathrm{~B}$ & 8.7 & 6.2 \\
\hline U10642 & & 16 & 57 & 18 & -028 & $\mathrm{dE}$ & -5 & 20.3 & \\
\hline U11012 & $\mathrm{N} 6503$ & 17 & 49 & 58.7 & $700926 \mathrm{D}$ & $\mathrm{Sc}$ & $6 \mathrm{~A}$ & 8.7 & 6.2 \\
\hline E346-19 & N7424 & 22 & 54 & 28 & -412011 & $\mathrm{Sc}$ & $6 \mathrm{X}$ & 12.3 & 7.6 \\
\hline E291-14 & & 23 & 14 & 09 & -432153 & $\mathrm{Sc}$ & 6 & 9.3 & \\
\hline E408-09 & I5332 & 23 & 31 & 48 & -362241 & $\mathrm{Sc}$ & $7 \mathrm{~A}$ & 10.8 & 6.6 \\
\hline
\end{tabular}

${ }^{\mathrm{a}}$ The RC2 position of NGC 1337 is $0325.66-0833.8$. The converted diameter of NGC 1337 using the RC2 $T$ index of 6 is less than the $8^{\prime}$ selection limit.

NGC 3628. - The apparent extension to the south of this galaxy in the 60 and $100 \mu \mathrm{m}$ maps is a "hysteresis tail" in the forward scanning direction.

$N G C$ 4236. - The 60 and $100 \mu \mathrm{m}$ images appear as two pointlike sources, partially resolved in the cross-scan direction.

NGC 4244. - The $60 \mu \mathrm{m}$ image is similar to that of NGC 55; two off-nucleus pointlike sources are associated with emission knots in the optical image. The 60 and $100 \mu \mathrm{m}$ images are affected by Galactic cirrus.

NGC 4395. - The 60 and $100 \mu \mathrm{m}$ maps are similar to those of IC 2574; enhanced emission is coincident with optical emission knots to the southeast of the galaxy center. The faint optical arm to the north is seen in the $60 \mu \mathrm{m}$ map.

$N G C$ 4565.- Little detail is visible in the images of this edge-on system. Note that the alignment of the IRAS image is rotated slightly from the optical major axis because of the scanning direction.

$N G C$ 4656. - The emission from this galaxy is essentially composed of two point sources, one at the nucleus of NGC 4656 and the other to the northeast of the nucleus at the position of several emission knots seen in the optical image and the small companion irregular galaxy, NGC 4657.

NGC 5055.-The $100 \mu \mathrm{m}$ image may be affected by "hysteresis" effects on both sides of the galaxy.

NGC 5194 (M51). - The main features visible in the IRAS images are a broad plateau centered on M51 and a pointlike source at NGC 5195. There is some evidence for resolution in the in-scan direction of the main spiral arms of M51 at 12 and $25 \mu \mathrm{m}$. There is probably residual background emission due to Galactic cirrus in the 60 and $100 \mu \mathrm{m}$ images.

$N G C 5236(\mathrm{M} 83)$. - The images of this galaxy are rectangular, the resolution being inadequate to resolve more than a hint of spiral structure at 12 and $25 \mu \mathrm{m}$ in the high surface brightness disk. There is a small "hysteresis" problem in the $100 \mu \mathrm{m}$ image. The low-level spur in the $60 \mu \mathrm{m}$ image to the east of the nucleus is probably due to emission from a pair of background galaxies.

NGC 5457 (M101). - This is one of the most interesting of the infrared galaxy images presented in the atlas. Several discrete sources associated with $\mathrm{H}$ II regions are seen at 25 and $60 \mu \mathrm{m}$, superposed on a smooth disk. The optical arm to the northeast is partially resolved in the $60 \mu \mathrm{m}$ map.

NGC 6822. - This is an intriguing group 4 galaxy. Although the 60 and $100 \mu \mathrm{m}$ images are somewhat affected by Galactic cirrus, the measured infrared diameter of the galaxy is almost twice its optical diameter, as listed in $\mathrm{RC} 2$, corrected for Galactic extinction. Localized infrared sources coincident with $\mathrm{H}$ II regions are evident in the 12,25 , and $60 \mu \mathrm{m}$ maps.

$N G C$ 6946. - The IRAS images present a quite centrally concentrated appearance, with some evidence of arm resolution to the northwest. The discrete point source to the southeast is a background object unrelated to the galaxy. The shape of the point source reflects the different scanning angles of the component observations of the maps. A complex background at $100 \mu \mathrm{m}$ resulted in poor background removal.

The IRAS surface brightness maps of the galaxies of this study, written to tape in FITS format, are available as an IPAC product.

This catalog could not have been produced without the able assistance of the staff of the Infrared Processing and Analysis Center (IPAC). In particular, the authors thank Dr. J. Good for developing the two-temperature model, Drs. G. Helou and F. Boulanger for useful comments on this work, E. Barba for preparing the manuscript, and the IPAC Data Management Team members (G. Smith, G. Lairmore, J. Lampley, H. 
Hanson, R. Beck, E. Erwin, D. Jackson, L. Lloyd, and R. Urban) for their data processing support. We thank the Palomar Observatory (California Institute of Technology), the European Southern Observatory, and the Mount Wilson and Las Campanas Observatories (Carnegie Institution of
Washington) for granting permission to reproduce and display the optical photographs presented in the atlas. This research was supported by The National Aeronautics and Space Administration through the IRAS Extended Mission Program.

\section{APPENDIX}

\section{ADDITIONAL OPTICALLY LARGE GALAXIES LISTED IN UGC, MCG, AND ESO}

As discussed in $\S \mathrm{I}$, a major objective of this study is to report global IRAS flux densities of all observed galaxies likely to have large enough infrared extents to result in an underestimation of their integrated emission as reported in the PSC and the SSSC. RC2 makes no attempt at completeness, and the RC2 diameters are weighted means of diameters obtained from catalogs which, in some cases, predate more modern compilations of galaxy diameters. For these reasons, the merged catalogs of Nilson (1973, 1974, hereafter UGC and UGCA, respectively), Lauberts (1982, hereafter ESO), and Vorontsov-Velyaminov et al. (1962, 1963-1974, hereafter MCG) were searched for possible candidate galaxies not selected from RC2. (The merging of these catalogs to obtain nonredundant all-sky coverage is discussed in the Extragalactic Catalog.)

Diameters listed in these catalogs were converted to the $D_{25}$ scale of RC2 using the transformation equations reported in RC2 and ESO. Five large galaxies not contained in $\mathrm{RC} 2$ and one galaxy without a listed diameter in RC2 were found. Fifty-five galaxies listed in RC2 with diameters less than $8^{\prime}$ had converted diameters greater than the selection limit. These additional optically large galaxies are presented in Table 8. Column (1) is the catalog identification of the galaxy-the UGC (U) number, abbreviated ESO (E) designation, or MCG (M) name. Column (2) is the RC2 designation. Columns (3) and (4) give the optical equatorial coordinates (equinox 1950) as listed in the selection catalog or, for the coordinates followed by " $D$," from Dressel and Condon (1976). Column (5) is the morphological classification as listed in the selection catalog. The morphological classification as coded in RC2 as a $T$ index and bar parameter letter is listed in column (6). For the ESO galaxies not listed in RC2 the $T$ index is from ESO. Column (7) reports $D_{25}^{*}$, the selection catalog isophotal major diameter converted to the $D_{25}$ scale of RC2. The isophotal diameter as reported in RC2 is listed in column (8).

\section{REFERENCES}

Aaronson, M., et al. 1982, Ap. J. Suppl., 50, 241.

Burstein, D., and Heiles, C. 1984, Ap. J. Suppl., 54, 33.

Cataloged Galaxies and Quasars Observed in the IRAS Survey. 1985,

prepared by C. J. Lonsdale, G. Helou, J. Good, and W. Rice (Pasadena: JPL) (Extragalactic Catalog).

Chester, T. 1985, in Light on Dark Matter, ed. F. P. Israel (Dordrecht: Reidel), p. 3.

de Jong, T., Clegg, P. E., Soifer, B. T., Rowan-Robinson, M., Habing, H. J., Houck, J. R., Aumann, H. H., and Raimond, E. 1984, Ap J. (Letters), 278, L67.

de Vaucouleurs, G., de Vaucouleurs, A., and Corwin, H. G., Jr. 1976 Second Reference Catalogue of Bright Galaxies (Austin: University of Texas Press) (RC2).

Dressel, L. L., and Condon, J. J. 1976, Ap. J. Suppl., 31, 187

Gallagher, J. S., and Mould, J. R. 1981, Ap. J. (Letters), 244, L3.

Habing, H. J., et al. 1984, Ap. J. (Letters), 278, L59.

Helou, G., Khan, I., Malek, L., and Boehmer, L. 1987, Ap. J. Suppl., submitted.

Hodge, P. W. 1963, A. J., 68, 691. 1973, Ap. J., 182, 671.

Huchra, J. A., Davis. D., Latham, D., and Tonry, J. 1983, Ap. J. Suppl., 52, 89 .

Huchtmeier, W. K., Richter, O. G., Bohnenstengel, H. D., and Hauschildt, M. 1983, ESO Preprint No. 250.

Hummel, E. 1980, Astr. Ap. Suppl., 41, 151.

IRAS Catalogs and Atlases, Explanatory Supplement. 1985, ed. C. A. Beichman, G. Neugebauer, H. J. Habing, P. E. Clegg, and T. J. Chester (Washington, DC: GPO) (IRAS Supplement).

IRAS Point Source Catalog. 1985, Joint IRAS Science Working Group (Washington, DC: GPO) (PSC).

IRAS Small Scale Structures Catalog. 1986, prepared by G. Helou and D. Walker (Washington, DC: GPO) (SSSC).

Kraan-Korteweg, R. C., and Tammann, G. A. 1979, Astr. Nach., 300, 181.

T. DE JoNG: Universiteit Amsterdam, Sterrenkundig Instituut, Roetersstraat 15, 1018 WB, Amsterdam, The Netherlands

H. J. Habing: Sterrewacht Leiden, P.O. Box 9513, 2300 RA, Leiden, The Netherlands

E. L. Kopan, Carol J. Lonsdale, L. A. Lloyd, and W. Rice: IPAC, Mail Code 100-22, California Institute of Technology, Pasadena, CA 91125

G. Neugebauer and B. T. Soifer: California Institute of Technology, Downs 320-47, Pasadena, CA 91125
Lauberts, A. 1982, The ESO/Uppsala Survey of the ESO(B) Atlas (Munich: European Southern Observatory) (ESO).

Low, F. J., et al. 1984, Ap. J. (Letters), 278, L19.

Neugebauer, G., et al. 1984, Ap. J. (Letters), 278, L1.

Nilson, P. 1973, Uppsala General Catalogue of Galaxies (Uppsala Astr. Obs. Ann., Vol. 6) (UGC). Rept., No. 5) (UGCA).

Rice, W. 1988, in preparation (Paper II).

Sandage, A., and Tammann, G. A. 1981, A Revised Shapley-Ames Catalog of Bright Galaxies (Carnegie Inst. Washington Pub., No. 635) (RSA).

Schwering, P. B. W. 1987, Astr. Ap. Suppl., in press.

Schwering, P. B. W., and Israel, F. P. 1987, Astr. Ap. Suppl., in press.

Soifer, B. T., Rice, W., Mould, J. R., Gillet, F. C., Rowan-Robinson, M.,

Soifer, B. T., et al. 1984, Ap. J. (Letters), 278, L71.

van den Bergh, S. 1959, Pub. David Dunlap Obs., 2, 147 (No. 5). 1966, A. J., 71, 922.

Verter, F. 1985, Ap. J. Suppl., 57, 261.

Vorontsov-Velyaminov, B. A., and Arhipova, V. P. 1963-1974, Morphological Catalog of Galaxies, Vols. 2-5 (Moscow: Moscow State University) (MCG).

Vorontsov-Velyaminov, B. A., and Krasnogorskaja, A. A. 1962, Morphological Catalog of Galaxies, Vol. 1 (Moscow: Moscow State University) (MCG).

Walterbos, R. A. M., and Schwering, P. B. W. 1987, Astr. Ap., in press. Young, E. T., Neugebauer, G., Kopan, E. L., Benson, R. D., Conrow, T. P., Rice, W., and Gregorich, D. T. 1985, A User's Guide to the IRAS Pointed Observation Products, IPAC Preprint Pre-0008N (POG).
Sanders, D., and Young, J. 1986, private communication. and Habing, H. J. 1986, Ap. J., 304, 651. 